University of Louisville

ThinkIR: The University of Louisville's Institutional Repository

Electronic Theses and Dissertations

$12-2010$

\title{
Factors affecting nurse educators' decision to teach.
}

Linda J. Thomas 1946-

University of Louisville

Follow this and additional works at: https://ir.library.louisville.edu/etd

\section{Recommended Citation}

Thomas, Linda J. 1946-, "Factors affecting nurse educators' decision to teach." (2010). Electronic Theses and Dissertations. Paper 1429.

https://doi.org/10.18297/etd/1429

This Doctoral Dissertation is brought to you for free and open access by ThinkIR: The University of Louisville's Institutional Repository. It has been accepted for inclusion in Electronic Theses and Dissertations by an authorized administrator of ThinkIR: The University of Louisville's Institutional Repository. This title appears here courtesy of the author, who has retained all other copyrights. For more information, please contact thinkir@louisville.edu. 


\title{
FACTORS AFFECTING NURSE EDUCATORS' DECISION TO TEACH
}

\author{
By \\ Linda J. Thomas \\ B.S.N., University of Kansas, 1969 \\ M.S.N., University of Evansville, 1985
}

\begin{abstract}
Dissertation
Submitted to the Faculty of the

Graduate School of the University of Louisville and Western Kentucky University In Partial Fulfillment of the Requirements for the Degree of
\end{abstract}

Doctor of Philosophy

Department of Education

University of Louisville

Louisville, Kentucky

Department of Education and Learning

Western Kentucky University

Bowling Green, Kentucky

December 2010 



\title{
FACTORS AFFECTING NURSE EDUCATORS' DECISION TO TEACH
}

\author{
By
}

Linda Joyce Thomas

B.S.N., University of Kansas, 1969

M.S.N., University of Evansville, 1985

A Dissertation Approved on

November 30, 2010

By the following Dissertation Committee:
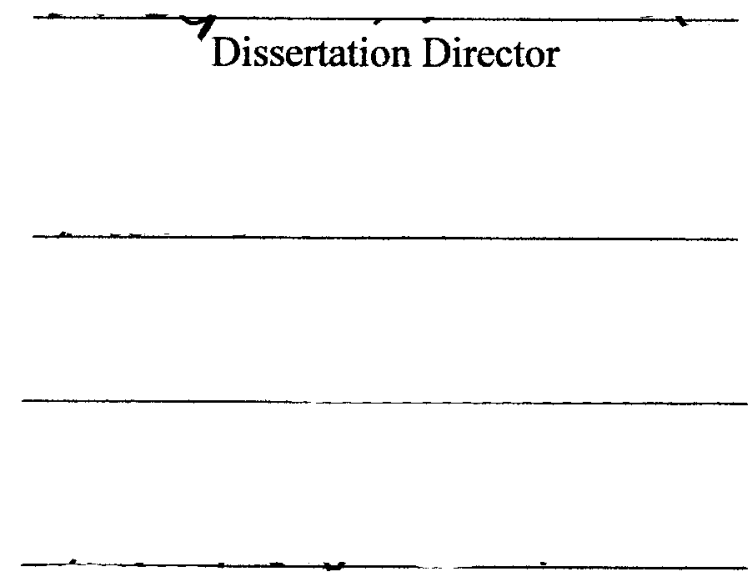


\section{ACKNOWLEDGMENTS}

I would like to thank my Dissertation Chair, Dr. Steve Miller, for his guidance, perseverance and for being the primary motivator to allow me to complete this journey. I would also like to thank my other committee members, Dr. John Keedy, Dr. Gayle Ecton, Dr. Paulette Adams, Dr. Donna Blackburn, and Dr. Beverly Siegrist, for their comments, suggestions and support over the years. I would like to especially acknowledge my

husband, Bob, for his support and his contributions to the dissertation and the travels along the way. Also, many thanks to my children, Stephanie for her continued support, for Bryan who provided valuable assistance when it was needed, as well as Chris and Drew. To my Mother, who never thought I would complete the work. To my fellow nursing faculty and staff members who provided the assistance and support for whatever I needed, you all were so generous. For all of those who gave of your time and efforts we all share in this celebration. 


\section{ABSTRACT \\ Factors Affecting Nurse Educators' Decision to Teach}

Linda J. Thomas

November 30, 2010

The field of nursing currently faces a shortage of both nurses and nursing faculty. Yet, little is known about why clinical nurses opt to become nursing faculty. Qualitative investigations into this phenomenon are particularly rare. This study addressed these gaps in the context of career choice of faculty associate degree nursing (ADN) programs. The qualitative analysis was guided by Astin's (1984) psychosocial model of career choice. The central research question was, "What factors influence the career choice of nurse educators currently teaching in Kentucky associated degree nursing programs?"

Faculty from the $20 \mathrm{ADN}$ programs in public community colleges and 4-year institutions in Kentucky were studied. Two instruments were developed: Background Questionnaire for Nurse Educators (BQ) and Nurse Educator Interview Schedule (IS). Using purposeful sampling, one individual from each program comprised a matrix representing educational attainment, years of nursing faculty experience, and college size. Responses from semi-structured interviews were recorded and transcribed. Data for the 19 participants were entered into a Coded Master Analytical Tool (43 interview questions and 10 background survey items). Faculty interviews were synthesized into the Summarized IS Response Matrix and analyzed for concepts, themes, and patterns.

Results were organized by the seven research questions and subquestions, mapped to the BQ and IS. Several findings had not been previously noted in the literature. Only the 
most prominent findings are noted here, including: (a) flexibility and role autonomy are key reasons for moving from clinical nursing to educator role; (b) faculty are sustained by psychic rewards of student relationships and success; (c) feedback from fellow faculty, administrators, and students is valuable; (d) new faculty lacked confidence in lecture preparation, examinations, and curriculum development, were comfortable with clinical evaluation and technology; (e) promotion/tenure and external service were confusing and negatively perceived; (f) love of teaching and lifelong learning were important aspects of their job; and (g) these different benefits compensated for lack of salary parity. The 19 responses resulted in few differences across the subgroups for educational attainment, years of nursing faculty experience, and college size. 


\section{TABLE OF CONTENTS}

PAGE

ACKNOWLEDGEMENTS

ABSTRACT

CHAPTER

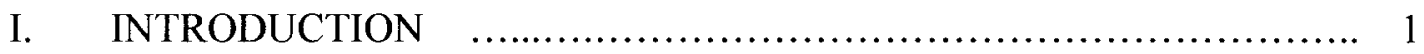

The Research Problem $\quad \ldots \ldots \ldots \ldots \ldots \ldots \ldots \ldots \ldots \ldots \ldots \ldots \ldots \ldots \ldots . \ldots \ldots$

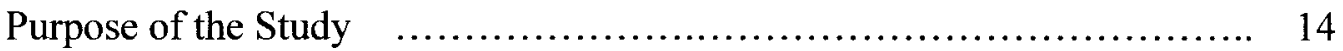

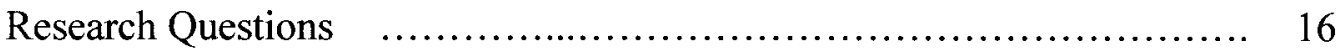

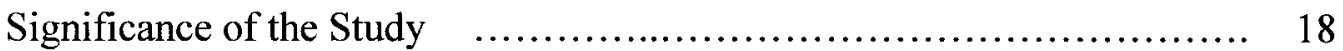

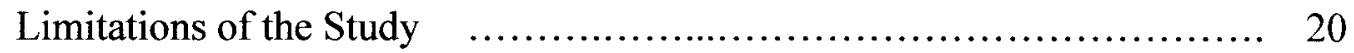

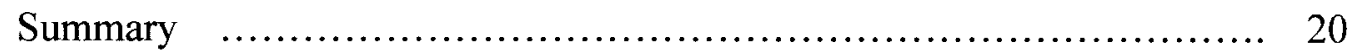

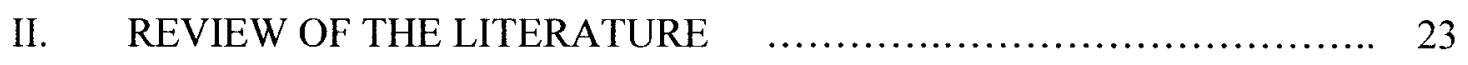

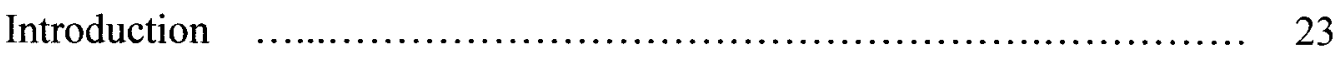

Historical Perspectives in Nursing Education $\quad \ldots \ldots \ldots \ldots \ldots \ldots \ldots \ldots \ldots . \ldots . \ldots . \ldots 23$

Research Studies in Nursing Education $\quad \ldots \ldots \ldots \ldots \ldots \ldots \ldots \ldots \ldots \ldots \ldots . \ldots \ldots \ldots \ldots$

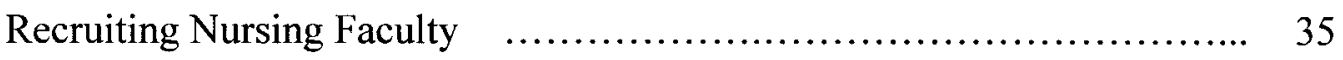

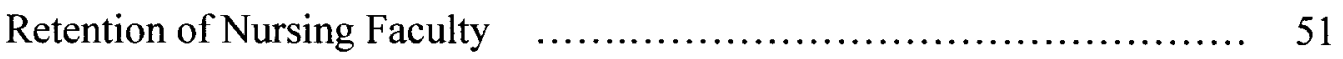

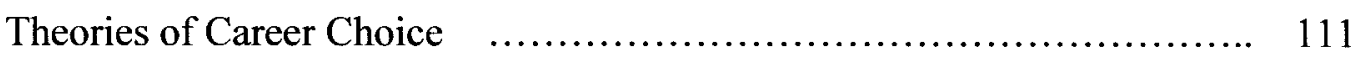

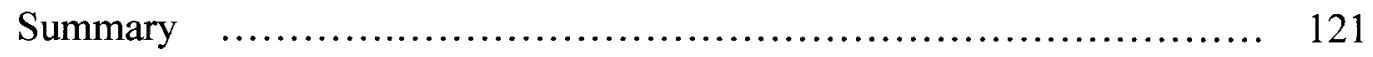

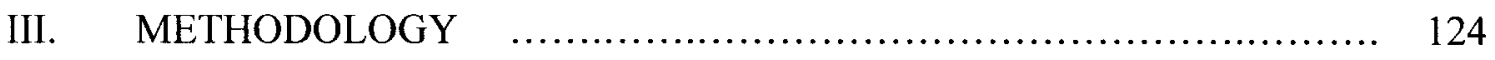

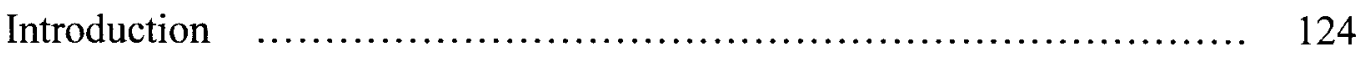

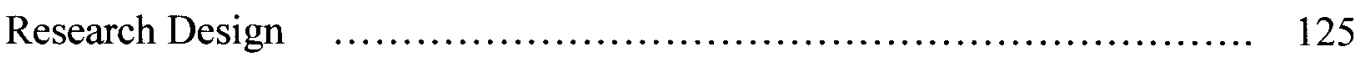




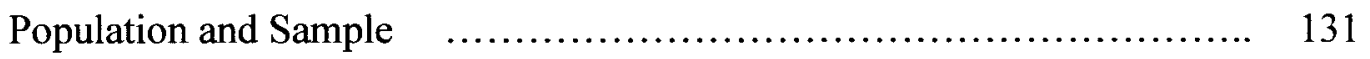

Research Questions $\quad$...................................................... 133

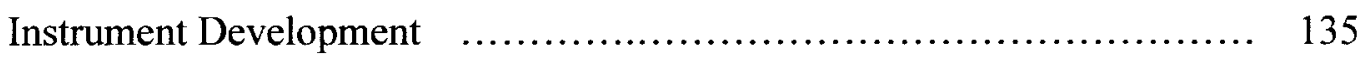

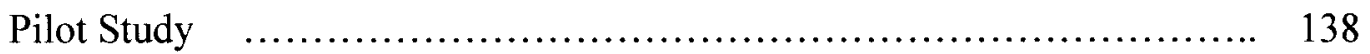

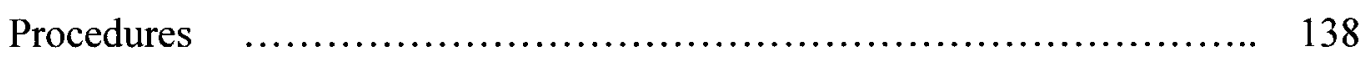

Data Analysis $\quad$...................................................... 141

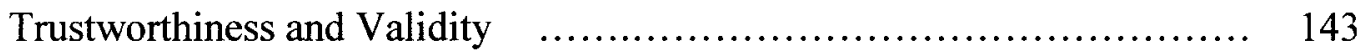

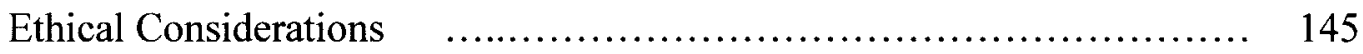

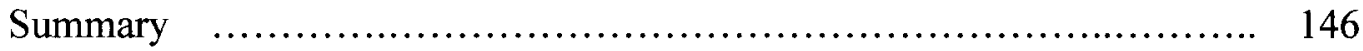

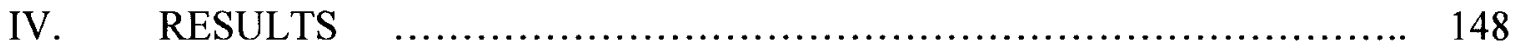

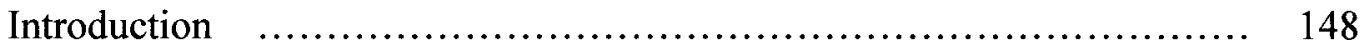

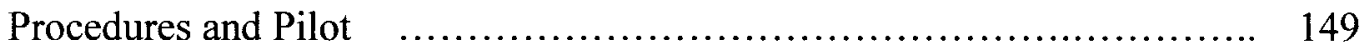

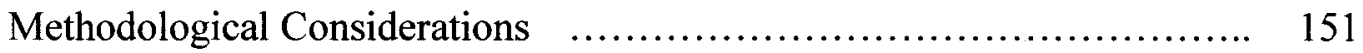

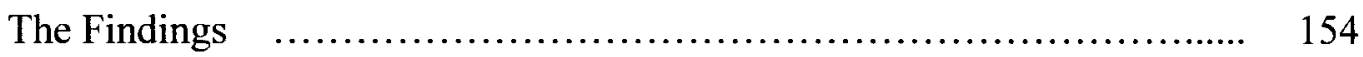

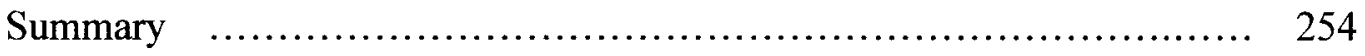

V. DISCUSSION AND CONCLUSIONS _................................................ 255

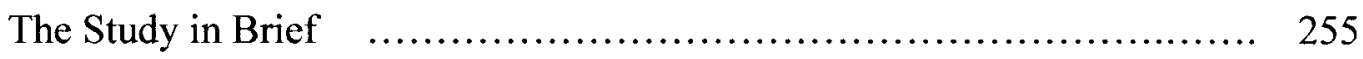

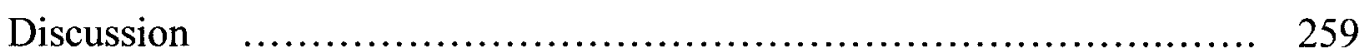

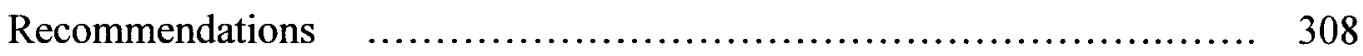

Future Research .................................................... 311

Conclusions $\quad$............................................................... 313

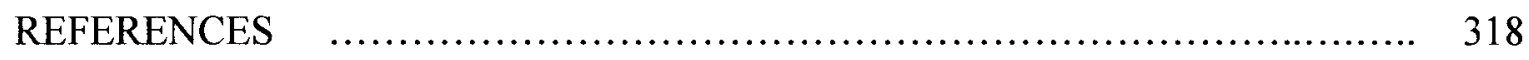

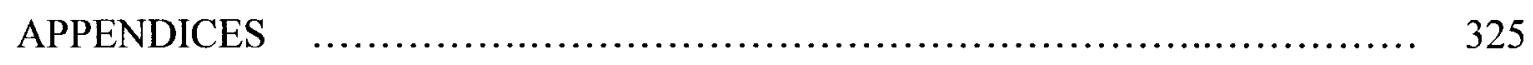

CURRICULUM VITAE $\quad$.................................................. 373 


\section{CHAPTER I \\ STATEMENT OF THE PROBLEM}

Introduction

As the nursing profession enters the 21 st century, a serious challenge emerges, that of supply and demand. As healthcare continues to demand more nurses, the education of those nurses is crucial. Nursing programs to educate tomorrow's nurses require a cadre of nursing faculty who themselves are being depleted by retirement, or are being lured into advanced clinical practice to meet today's healthcare needs. The lack of nurses educated to give quality nursing care is a major concern to the nation. As more nurses are needed, there will be a need for more faculty to provide the education to prepare a knowledgeable and caring individual to meet today's healthcare needs.

According to the National Sample Survey of Registered Nurses (RN's) (United States Department of Health and Human Services) in 2000, there were 2,694,540 persons estimated to have licenses to practice as registered nurses in the United States. From 1980 to 2000 there was an increase of $62.2 \%$ in nursing graduates. This was the slowest growth in the $\mathrm{RN}$ population of any 20 -year period. On average, the $\mathrm{RN}$ population grew $1.3 \%$ each year between 1996 and 2000. If the profession grows at the present rate, by 2010 , there will be one million vacant registered nursing positions (American Association of Colleges of Nursing [AACN], 2003). The National Sample Survey also reports a continuing trend in the aging of the RN population. In $1980,25.1 \%$ of the RNs were 
under the age of 30 ; in 2000 there were only $9.1 \%$. In 2000 the average age the $\mathrm{RN}$ population was 45.2 years, as compared to 44.3 years in 1996.

Men in nursing comprise $5.4 \%$ or 146,902 of the $\mathrm{RN}$ labor force. In twenty years the number of males in the profession has increased $226 \%$. Surveys indicate that the number of men going into nursing has grown at a much faster rate than the total RN population. Still, men are choosing to go into clinical nursing positions rather than educator roles. Demographic data from population samples of research studies confirm that very few men are currently teaching in any type of nursing program (AACN, 2003).

The growth of minorities in nursing has also been rapid, although not in keeping with the proportionate growth of minorities in the total population. The number of minority nurses grew $35 \%$ percent during the four-year period of $1996-2000$, while the nonminority population grew 2\%. During the period from 1996-2000, Hispanic/Latino nurses had the highest growth in numbers $(12 \%)$, more than any other minority group. However, the growth of minorities in nursing education continues to lag behind that of Caucasian faculty (AACN, 2003).

Buerhaus, Staiger, and Auerbach (2000) conducted an investigational study to identify and assess key sources of changes in age distribution and total supply of RNs looking at the year 2020, to project the future age distribution and the total RN workforce. Looking at workforce trends of $\mathrm{RN}$ cohort groups, within the next 10 years, the average age of the RN workforce would be 45.4 years, an increase by 3.5 years over the current age.

Despite the economic recession where jobs were being lost in record numbers, jobs in healthcare continued to increase. In June 2009, 21,000 jobs were created in the healthcare industry including hospitals, long-term care facilities, and ambulatory 
healthcare settings. Overall, the healthcare industry has added 544,000 jobs since the beginning of the recession (AACN, 2009).

\section{The Research Problem}

There is minimal research dealing with nursing faculty and their career choice, despite the changing conditions within the collegiate setting in comparison to the clinical settings. As more nurses attain advanced degrees, the preparation for advanced clinical practice outweighs the demand and necessity for accepting nursing faculty positions. The workforce of nursing faculty is a graying one, with the average age of a faculty member above 50 years of age. More information is necessary to identify how the faculty workforce chooses nursing positions. Specifically, there is little research on the reasoning of nursing faculty on why they selected a career in nursing education.

\section{The Nursing Faculty Shortage}

Other factors have been instrumental in the nursing faculty shortage. The Pew Health Profession Commission in 1995 issued recommendations for the nation's medical and nursing workforce to reform healthcare regulations for the 21 st century by providing better integration of services; using resources more effectively; becoming more innovative and diverse; and being more concerned with education, prevention, and care management and less focused on treatment. The commission called for the closure of hospitals and predicted a surplus of doctors, nurses and pharmacists to meet the demand-driven system of health care. The commission addressed nursing by challenging the profession to recognize multiple entry levels for professional practice (licensed practical nurse, associate degree $\mathrm{RN}$, and baccalaureate $\mathrm{RN}$ ), and encouraged the expansion of master's level nurse practitioner training programs to assist in the provision of better health care. As a result, federal support for students in nurse practitioner programs increased the enrollment in 
master's programs, at the sacrifice of programs preparing nurse educators.

Hinshaw (2001) looked at the nursing faculty shortage from a historical perspective. In the 1980 s there was a decline in the nursing program enrollments and as a result faculty positions were reduced. With the increase in demand of nurses beginning in the 1990s, new programs opened and others increased their numbers. Faculty found other opportunities within the nursing profession. In response, part-time faculty were hired to ease the shortage. Hinshaw also concluded that nurses with a graduate education have more attractive choices other than academic positions. Nurses have moved into corporate and administrative roles and have not developed opportunities for attaining the skills to teach.

Educational requirements for nurse faculty positions maintain that the nurse educator be master's prepared or higher. Once graduates of nursing programs at the baccalaureate, diploma, or associate degree level become licensed and begin working, eligibility for graduate nursing programs requires the nurse to gain experience in the clinical setting, then move into higher education. This then creates an older candidate eligible to teach.

The lack of relatively young faculty members can be attributed to, in part, the length of time to acquire the requisite education, but it is also a function of academic administrators' traditional views in mandating "years of experience" prior to entry into masters and doctoral programs. We force out graduate students to postpone furthering their academic preparation and push the clock later before they are ready to fulfill the academic roles, where peers in other disciplines have earlier starts. The reality is, therefore, that our careers get a later start and yield shorter academic life in educator positions. (Feeg, 2004, p. 8)

Students completing nursing doctoral degrees in 1999 had a reported age of 46.2 years (AACN, 2003). Over $48 \%$ of the graduates were between the ages of 45 and 54 years, and $12 \%$ were over 55 years. There were only $25(6.8 \%)$ of 365 graduates under the 
age of 35 . The average age of full-time nursing faculty retiring in 2002 was 61.5 years; therefore, the length of a teaching career could be from 6-15 years.

Of 76 schools reporting data to the American Association of Colleges of Nursing over a four-year period from 1998-2002, there was an average increase of 31 doctoral students per year. However, graduation rates had not increased. Enrollment of doctoral students in the fall of 2002 in 81 programs in nursing was 3,168 students. There were only 457 graduates in that time period (AACN, 2003). The elapsed time from completion of a master's degree to doctoral degree was 15.9 years for nursing, 8.5 years for other fields.

In a descriptive study conducted by the Southern Regional Education Board (SREB) in 2000 to collect information on the national supply of nurses, surveys were sent to 491 nursing schools in the southern region of the United States. Surveys were completed and returned from 392 programs: 152 associate degree programs, 130 bachelor degree programs, 81 masters, and 29 doctoral programs, a 56\% return rate. Most nursing programs were located in rural settings (35\%) followed by $21 \%$ of the programs located in small cities with a population of 50,000 to 99,000 .

There were 6,382 full-time and part-time faculty positions at the beginning of the 2000-2001 school year and 314 unfilled full-time and part-time positions. At the close of the academic year there were 432 unfilled positions (306 full-time and 126 part-time). There were 4,302 full-time faculty and 1,246 part-time with nearly 500 full-and part-time nursing educators not holding the minimal credential (a Master's degree in nursing) (SREB, 2000).

Resignations reduced the workforce in nursing education in the region as 72 faculty with Doctorates resigned during 2000-2001; this was four percent of all faculty with Doctorates. Two hundred and forty-nine of those who resigned had master's degrees and 
represented six percent of all faculty with master's degrees. Reasons for the resignations included family responsibilities (care for an elderly parent), career advancement, and salary. The SREB (2000) authors suggested further research would be valuable, focusing on reasons faculty resign, and the development of effective plans for faculty retention.

In the SREB (2000) study, retirements during the 2000-2001 academic year totaled 144 nursing educators. The average age of a faculty member was $58.5,13$ years older than the average age of an $\mathrm{RN}$ in the present workforce. Of those that retired, 55 had Doctorates in nursing and 89 had master's degrees. Explanations for the increasing age of the RN population are believed to be caused by a variety of factors: demographic, social and educational. In the period between 1960 and 1970 there was a larger group of women who chose nursing than the number of individuals currently graduating from nursing programs. The recent expansion of career opportunities and the increase in earnings in other fields has resulted in women choosing other careers.

In a descriptive study, Craine (2000) examined the nurse faculty supply and demand statewide in Georgia. Using a qualitative format, a survey was sent to 30 registered nursing degree programs for completion by nursing faculty. Twenty-seven schools, a $90 \%$ response rate, responded and were representative of all levels of nursing programs. Faculty, department chairs, Master's level graduate students, and graduate coordinators of Master's programs completed the questionnaire $(N=488)$. The results were similar to the SREB report. There was a six-year decline in the average number of nursing faculty and students. The mean faculty age was 51.5 years, which exceeded the national average of 50 years for educators. The survey also revealed that $18 \%$ of the faculty who responded to the questionnaire planned to retire within the next five years, and an additional 245 or $23 \%$ would retire within the next six to ten. Without qualified 
candidates to replace the retiring educators, Craine predicted that there would be a total of only 145 faculty to teach in 27 programs across the state. This would result in each program enrolling fewer students.

Of the 246 faculty who returned the questionnaire, $53 \%$ responded that they chose education for reasons of altruism and personal satisfaction. Twenty-three percent of the faculty chose teaching for the autonomy associated with the position, $3.7 \%$ for job security, and $2 \%$ chose the educator role based upon salary (Crane, 2000). Further, surveys from 315 respondents enrolled in the graduate programs revealed that only four $(2 \%)$ of 169 students planned to teach after graduation. The remainder of the group $(87 \%)$ intended to work as advanced nurse practitioners.

Brendtro and Hegge (2000), in a descriptive study, surveyed nurses with graduate degrees in one Midwestern state as part of a nursing workforce project. Research questions that framed the study were: (a) how does the age of nursing faculty compare with the age of graduate nurses employed in nonacademic settings? (b) what positions do nurses with graduate degrees currently hold and how satisfied are they with those positions? (c) what incentives could be employed to attract and retain nursing faculty, and (d) what ideas do graduate nurses have for increasing numbers of qualified nurse educators?

The survey instrument, containing questions dealing with demographics was sent to all nurses with graduate degrees within the state. The study sample $(N=288)$ had a $61 \%$ response rate. Results indicated that nursing faculty reported significantly lower annual salaries $(\$ 20,000$ to over $\$ 100,000$; with a mean salary of $\$ 43,200)$ as compared to nurses in clinical practice ( $\$ 61,000$ to 93,000$)$. The respondents perceived barriers that prevented them from pursuing advanced education; these included: inconvenient location, 
inappropriate focus for intended career goals, financial drains, family commitments, lack of time, and satisfaction with their current level of education. As incentives for recruiting faculty, the respondents suggested improving compensation, more realistic professional expectations, closer distance to work, and increased opportunities to continue clinical practice.

In 1993, the Kentucky Board of Nursing changed the regulation of the student/faculty ratio in the clinical area not to exceed a maximum of ten students to one faculty member in a clinical practice experience. Faculty, prior to that time, had been governed by a 12 to 1 ratio in the clinical setting. The reduction in the number of students for one faculty member in the clinical setting created the demand for more nursing faculty, or reduction of the number of students admitted to the programs.

In Kentucky there are three colleges that offer a doctoral degree in nursing. Two of the three colleges (University of Kentucky and Frontier School of Midwifery) offer a Doctorate in Nursing Practice (DNP). The University of Kentucky and University of Louisville offer the Doctor of Philosophy in Nursing ( $\mathrm{PhD}$ in Nursing). The DNP prepares nurses for clinical and executive leadership. The $\mathrm{PhD}$ in nursing prepares graduates for academic research and educational positions. Nursing faculty may also attain either the EdD or PhD in Education.

There are 11 colleges that offer a Master of Science in Nursing (MSN) degree. The MSN is the minimal educational requirement for teaching in an associate degree or baccalaureate nursing program. There are 28 programs preparing graduates at the $\mathrm{RN}$ level, and there are 27 programs preparing Licensed Practical Nurse (LPN) graduates (Kentucky Board of Nursing, n.d.) With the declining financial situation in the state of Kentucky as well as other states, funding has been decreased to the colleges. With no new money to the 
schools, only through private monies or the retirement or resignations of faculty in other positions, have new faculty positions been funded.

Ryan and Irvine (1994) in another study examined current and projected faculty profiles, policies related to retirement, and projected replacements for schools of nursing for the next 15 years. With private and public educational systems grappling with financial concerns that have an impact on availability of economic resources, neither state nor institutional resources may be forthcoming to support current replacement or additional faculty needs. Anticipated retirements may lead to a significant turnover within the nursing professorate. Competitive salaries and flexible work schedules in nursing practice areas are luring nurses with advanced degrees away from academe. Irvine and Ryan used the term "bipolar professorate" to characterize the senior and junior faculty in nursing education. New faculties need the assistance of a mentor and coach, while more mature faculty need assistance with retirement plans and options for careers. The researchers posed questions concerning: (a) what faculty characteristics were representative of responding institutions, (b) what policies were in place related to retirement, (c) what were projected patterns of retirement over the next 10 years, (d) what recruitment had been done in the past 10 years, and (e) how many master's and doctoral level prepared nurses were expected to graduate in the next five years and what were the specialties of those candidates.

Questionnaires were mailed to all baccalaureate and higher degree programs accredited by the National League for Nursing, producing a final sample of 296 , a $58 \%$ return rate. The peak age range was $42-50$ years of age (43\%). Twenty-eight percent of the faculty were 40 years and younger, and $24 \%$ were 51 years and older. In response to the availability of qualified applicants, $57 \%$ of the respondents reported a decline in qualified applicants, as well as a difficulty in finding faculty for specific areas. Fifty percent of the 
administrators noted a problem in finding faculty for pediatrics and maternity nursing. Academic cutbacks and successful recruitment of master's level faculty by hospitals were also considered as factors (Ryan \& Irvine, 1994).

In a white paper on Public Policy Initiatives in 2002, the Joint Commission on Accreditation of Healthcare Organizations (JCAHO), the national group overseeing hospitals, addressed strategies for overcoming the nursing crisis including nursing education. The organization cited the age difference of a nursing school faculty member (average 51years) as being eight years older than the bedside nurse. Doctorally prepared faculty averaged 56 years of age. The AACN (2003) stated that from 2000-2001 nursing enrollments in doctoral education programs grew only by $1.5 \%$. Doctoral programs are the main supplier of nurse educators for baccalaureate programs and above. The organization cited the lack of competitive salaries in education as opposed to clinical and private research settings.

JCAHO (2002) cited the lack of federal funding for nursing education in comparison to medical education. In $2001, \$ 78$ million was appropriated to fund basic and advanced nursing education and included scholarships for disadvantaged and minority students. Federal funding for medical education in the same time period was $\$ 9$ billion for payment to hospitals in 2002 , not including other forms of reimbursement. The Commission cited the need for private funding as well as the endowments by individuals to nursing schools to increase funding for nursing faculty and students.

In the AACN (2003) report, 27\% of the graduates from doctoral programs in 2002 planned to assume positions other than in nursing education. Salaries for faculty with doctoral degrees with a rank of associate professor were $\$ 74,556$; for nursing faculty with master's degrees the median salary was $\$ 60,556$. Median salaries of the clinical or 
administrative counterpart for a VP of nursing were $\$ 128,879$ and $\$ 93,344$ for a nursing director. Academic institutions cannot compete with private service or administrative positions, especially given budgetary concerns, and the master's prepared nurse is not attracted to returning to school for a doctoral degree. Salaries are higher in a non-academic setting.

The AACN (2003) survey reported an enrollment of 3,168 graduate nursing students in doctoral programs and only 457 graduates. Fifty-five percent of the students enrolled were part-time. This part-time status delayed the number of students who would complete the degree and be available to teach. In the same study, nursing master's programs for a five year period from 1998-2001 reported a decline in enrollments. But despite an increase of 898 students in 2002, the authors anticipated an overall decline in the number of graduates due to the delayed entry of the students into the ranks of nursing faculty. Additionally, these students may already be currently employed as nursing faculty in educational programs.

From the previous studies, the aging RN workforce as well as the decline in faculty positions depicts a crisis in nursing education. As the demands for nurses rise and the numbers of nursing educators leave the profession through retirement, education must be able to provide well-qualified faculty to meet the challenge. Recruitment of faculty will be of most importance. There is little empirical research on recruitment of nursing faculty; however, in a somewhat parallel situation, community college officials are faced with many of the same concerns as nursing. Large numbers of community colleges proliferated in the 1960s and many of the faculty hired at that time still remain at the institution, but will soon retire.

Recruiting Nursing Faculty 
AACN (2003) identified strategies to attract more nurses to educational positions. The organization advocated for opportunities to sponsor educational sessions to appeal to nurses in practice by emphasizing the positive aspects of the teaching role and seeking assistance to gain the important credentials required of faculty. AACN also advocated for reducing or eliminating nursing experience as a requirement for graduate study. By doing so, younger nurses could move from initial nursing programs to advanced study at an earlier age. By examining curricula of graduate programs to facilitate a more rapid completion of graduate study, nurses could complete requirements and enter the educator ranks sooner. By attracting second-degree students from other fields into the nursing profession and identifying students who exhibit superior performance early in their education, there could be encouragement for the academic role. Faculty who identify their role in academe as rewarding and satisfying should be encouraged to meet with interested nurses to attract others to the role.

\section{Research on the Nursing Shortage}

Choosing to teach nursing, according to Feeg $(2004$, p. 8), is "demanding work that requires a particular kind of knowledge and talent beyond the mastery of the psychomotor or technical skills that are developed for the jobs our students will fill." The work of faculty includes preparation of content for presentation, staying abreast of the latest nursing theory, grading paperwork in a timely fashion, and handling novice and beginner students in a highly-charged clinical environment. "We measure our successes by watching the fledglings in our charge evolve to become competent clinicians who will care for patients" (Feeg, p. 8).

Higgins and Hawthorne (1994) developed a model to recruit individuals who were considering teaching at two-year colleges as a viable and attractive first-choice career. 
Subjects were identified after 130 Chief Academic Officers of publicly supported two year colleges in Illinois identified five full-time faculty who had demonstrated a commitment to their institution's mission. Through identification of behaviors, a portrait of a successful faculty member was developed to recruit prospective faculty. Of 600 letters that invited participation, 289 responded for a $48 \%$ response rate. The ages ranged from $28-72$, with $50 \%$ of the subjects between 45 and 54 years of age. Completion of a survey was required using a 5-point Likert-type scale measuring satisfaction or dissatisfaction in relation to their careers. They were asked to choose three most important reasons out of 13 given for teaching at the community college level, and to rate characteristics for successful teaching at the college and activities important to the mission of the college. Survey respondents rated classroom teaching as the most important reason for remaining at the college $(76 \%)$. The greatest majority of the respondents had worked in business and industry prior to teaching full time. Ninety-four percent of the respondents rated communication skills as most important for teaching.

After administration of the questionnaire, Higgins and Hawthorne (1994) interviewed a cross-section of subjects (20) selected by geographic location, type of institution, demographic data, and completeness of responses on the questionnaire. Interviews were conducted by telephone and lasted approximately one hour. The interviewees identified what factors were important in their decision to teach in a two-year college. Less than half of the subjects had decided during or shortly after graduate school to pursue teaching at the community college. Only one nurse had indicated going to graduate school specifically for an educational background to teach. Respondents also rated 12 suggested characteristics for successful teaching at the two-year college level. Communication skills were the most important (94\%), followed by the ability to relate to 
students $(93 \%)$, interpersonal skills $(88 \%)$, organizational skills $(69 \%)$, and a degree in one's teaching discipline $(63 \%)$. Forty-eight percent of the students ranked the training in teaching and learning as somewhat important.

Winter and his associates (Winter, 1996; Winter, 2000; Winter \& Kjorlien, 2000; Winter \& Munoz, 2001) conducted several studies with graduate students to examine what factors would attract applicants to apply for academic positions. Although the subjects were not in the field of nursing, comparisons to the studies and the results could provide insight in recruitment. Recruiter similarity, marital status, required hours on job and applicant gender were found to be important to attract students to apply for faculty positions.

For example, Winter and Munoz (2001) used a recruitment simulation with experienced business professionals, enrolled in an MBA program, whose task was to roleplay the part of job applicants for a community college setting. The 194 subjects were required to complete a biographical data form, a job satisfaction instrument, and then rate a business faculty job vacancy detailed in a simulated recruitment advertisement. Results indicated that marital status was the independent variable that correlated the highest in explaining the variance of the applicants' ratings of the faculty position. There was no significant correlation between the dependent variables and (a) gender, (b) race, (c) business experience, (d) job tenure, and (e) job satisfaction. When the two variables marital status and hours worked per week were entered into a step-wise regression equation, marital status was the independent variable that made the highest contribution to explaining variance in applicants' job rating (5\%), with a negative correlation between marital status and applicants' job rating. Therefore, participants who were not married had increased attraction to the job. 


\section{Purpose of the Study}

The literature dealing with the nursing faculty shortage discusses what factors are operating to create the crisis and the implications of the situation. Introduced in the preceding section, data relating to these issues have been collected from a variety of sources, looking at the current supply and pending shortage based upon the "graying" of the nursing population. Statistics examining the current salary conditions and atmosphere of academe point to a further dwindling of resources (These studies are detailed in Chapter II, Review of the Literature).

The issues laid out above point to both a lack of nursing faculty and an incomplete understanding of why nurses in the field choose to make the switch to higher education as nursing faculty. In particular, most of the related studies focus on demographics or constitute quantitative analysis of state or federal data. Noticeably absent are qualitative analyses that address the reasoning or feelings of nursing faculty related to their current jobs or why they changed careers. Consistent with this emphasis on numbers rather than personal explanations for the trends observed, much of the literature on the nursing faculty is atheoretical.

This study addressed these gaps in the context of the career choice of nursing faculty in the state of Kentucky. Because there are multiple levels of nursing education, from nurse aid to licensed practical nurse (LPN) to associate degree and baccalaureate degree nurses (ADN and BSN, respectively), this study was limited only to nursing faculty in associate degree nursing programs. Perhaps these highly trained and experienced educators can provide insights into their choice regarding working in academe, the role they play, and the possible solutions to recruiting other dedicated nurses.

Specifically, the purpose of this study was to provide insight into the career choice 
of associate degree nursing faculty. The analysis was qualitative, guided by a theoretical framework (Astin, 1984) to explore faculty thoughts, dispositions, and reasoning about their role in higher education and why they changed from clinical practice to teaching. Consistent with this emphasis, the central research question was, "What factors influence the career choice of nurse educators currently teaching in Kentucky associate degree nursing programs?"

\section{Research Questions}

There is currently great concern among the nation's educational leaders on meeting the needs of nursing education and the dwindling supply of manpower available as higher education faculty in this field. Increased attention has centered on the career choice of nurses to teach. The shortage of nursing faculty is intertwined with the shortage of nurses. As educational institutions scurry to increase their numbers enrolled in nursing programs, the lack of available nursing faculty affects the number that can be educated. As the numbers decline in nurses completing programs, the numbers available to choose teaching are dwindling as well (Hinshaw, 2001).

Although the nursing faculty shortage is national in scope, strategies to address the problem require solutions at both national and state levels. Initiatives to increase awareness of the issues and to increase attractiveness of nursing as a profession are sorely needed (AACN, 2009). Yet specific programs and policies will have to be set at the state level because of state funding formulas for higher education.

Therefore, the following questions gave structure to this investigation on what factors influenced the career choice of nurse educators currently teaching in Kentucky in associate degree nursing programs.

1. To what extent are personal and demographic factors influential: 

a. Age?
b. Marital status?
c. Children or other family responsibilities?
d. Years of nursing experience?
e. Years of nursing faculty experience?
f. Family educational attainment?
g. Educational attainment?
h. Influential person or event?
i. College--Small or Medium/Large?
j. Other?

2. In what ways do educational degree requirements for faculty positions:
a. Influence decisions to become a faculty member?
b. Influence career trajectory (tenure and promotion) and retention?

3. To what extent is the working environment in academia (as opposed to clinical nursing) influential:
a. Flexibility?
b. Collegiality?
c. Organizational climate?
d. Prestige/status?
e. Other?

4. To what extent are pedagogical considerations influential:
a. Love of teaching?
b. Lifelong learning?
c. Possibility of influencing the next generation of nurses? 

d. Content knowledge and skills?
e. Other?

5. What are the expectations for "being nursing faculty" when first hired regarding:
a. Teaching load?
b. Service?
c. Scholarly writing and research?
d. Earning tenure and/or promotion?

Beyond specific issues already addressed:

6. What are the primary barriers to becoming a nurse educator?

7. What are the primary satisfactions of working as a nurse educator?

\section{Significance of the Study}

The literature describes the nursing faculty shortage, and statistics provide data that forecast worsening conditions as hospitals and other healthcare facilities demand more nurses. The education of these caregivers must come from those nursing faculty who have been trained and who aspire to impart the knowledge, skills, and science of nursing.

This study provided information about factors identified by nursing faculty which determined their career choice in teaching. Semi-structured interviews as supplemented by a background questionnaire represented qualitative data on the shortage of nursing faculty. By interviewing a number of these educators, a rich description of their experiences, insights, and feelings may be gleaned. However, the investigation had several limitations.

First, this was a qualitative study to identify the factors that affect the nursing faculty members' decision to teach. Most of the nursing research cited has been quantitative in nature, examining job satisfaction, perceptions of the nursing role, attraction 
of faculty, and retention of faculty. Understanding what is important to the faculty member could provide additional information to assist in the recruitment and retention of additional nursing faculty members.

Second, the nature of the study focused primarily on the emic voice (the view of the individual from inside the teaching role). Because the researcher was a nurse educator with administrative responsibilities, maintaining some perspective of the etic (outsider's view) was important in the process of analyzing data (Danquah \& Miller, 2007; Gall, Borg, \& Gall, 1996).

Third, qualitative studies provided insights through the discovery of relationships or themes that can be examined more generally in subsequent studies. The interview process provides the opportunity for the subjects to expound on the subject at hand and the collection of those comments, thoughts, and ideas are the focus of the research itself (Vierra, Pollack, \& Golez, 1998).

Fourth, previous research studies on nursing faculty with a theoretical framework of career choice have been quantitative in nature. Qualitative research studies with a theoretical psychosocial career choice framework (Astin, 1984, Lindholm, 2004) have focused on educators but not those in nursing.

Fifth, this study addressed associate degree nursing faculty while previous cited research has been primarily from baccalaureate programs. Although there are more associate degree programs than baccalaureate nursing programs in the country, researchers have sampled BSN educators in greater numbers.

Finally, there is need for current research in the area of nursing faculty due to the faculty shortage in academe. The ability to increase the number of nurses entering the profession relies on the ability of programs to educate them. Faculty losses from retirement 
and resignation create a barrier to the enrollment of adequate numbers of nursing students.

Discovering what nursing faculty believe is important to promote the attraction and retention in their role and is important to the future of nursing.

$$
\text { Limitations of the Study }
$$

The current study, which explored the factors, including the barriers, that affect the nursing faculty's decision to teach, had several limitations. In general these were related to the nature of qualitative investigation and the particular context of the setting.

First, this study provided information on nursing faculty only in the state of Kentucky and does not represent the entire population of nursing faculty. Cost and time constraints limit the ability to interview nursing faculty from other states.

Second, this purposeful sample limited the number of subjects from each setting. Although all subjects constitute nursing faculty members who meet the specifications in the study, the findings may not be easily generalizable to the thoughts, beliefs, or experiences of all nursing faculty members.

Third, the study focused only on associate degree nursing faculty and did not provide information on LPN or BSN educators nor the differences across program levels. Certainly the nursing faculty shortage extends to all forms of nursing education.

Fourth, only the faculty role in nursing education was the focus of the study, not administrators, policy makers, or students. The nursing faculty shortage not only has an impact on the student but there may be additional key personnel (deans and administrators) who are affected by the shortage of nursing faculty.

\section{Summary}

The United States is currently experiencing a nursing shortage. There are several ramifications of this trend. The number of new graduate nurses needed to care for the 
increasing numbers of baby boomers moving into the elderly ranks is not keeping up with the actual numbers of students who are entering the profession. Attracting minorities into the profession is a challenge for educators to mirror the expanding minority population. Additional barriers to recruiting potential candidates into nursing are related to perceptions among young people making career plans that other professions may appear more attractive. With the reduced numbers of individuals graduating from nursing programs, older nurses may be pressed to continue working so that the average age of the nurse is increasing.

To resolve the continued nursing shortage, many nursing programs have proposed increasing the size of their classes. However, expanding capacity of nursing schools requires more nursing instructors which has contributed to a current shortage of nursing faculty. Exacerbating the situation is decreased financial support for education (a consequence of the current economic problems) and the opportunity for nurses with advanced education to choose employment within the clinical setting where there are greater financial rewards compared to higher education. Since advanced degrees are a prerequisite for teaching, the average age of the nurse faculty members is older than the nurse in the clinical setting. This situation decreases the actual duration of a faculty career, one more factor in the increasing number of positions unfilled in the nursing programs.

Given the realization that many of the present faculty members are within five years of retirement, additional concerns emerge for increasing the current number of nursing educators. The faculty shortage, the need to recruit and retain faculty is of utmost importance. Research studies in nursing and in other disciplines have provided a variety of strategies to attract nurses to faculty positions. The development of programs to assist the nurse faculty member in adapting to the changing role has been viewed as vital in the 
success and the longevity of the individual in the teaching role.

The nursing faculty shortage continues to influence the overall availability of nurses to care for the current and next generations, sick or well. Given the crucial situation in today's and tomorrow's healthcare, studying why nursing faculty from previous generations and with a variety of experiences actually made the decision to teach and continue to teach is the basis for this study. Thus the central research question was: "What factors influence the career choice of nurse educators currently teaching in Kentucky associate degree nursing programs?" 


\section{CHAPTER II \\ REVIEW OF THE LITERATURE}

\section{Introduction}

The purpose of this study is to identify factors affecting the career choice of nurse educators. Finding qualified nursing faculty is paramount in providing additional nurses to meet the healthcare needs of an aging population, both now and in the future. The literature review includes a brief history of the early education of nurses in the United States, a review of research studies in nursing education with a particular emphasis on recruitment of faculty (both nursing and non-nursing), retention of nursing faculty through the research on job satisfaction and burnout, orientation of new faculty, and theories of career choice. Through the identification of barriers that impact the role of the nurse educator and factors surrounding career choice, an understanding of the individual who makes the decision to teach should emerge.

\section{Historical Perspectives on Nursing Education}

When looking at nursing education, it is important to view the historical perspective of the practice of educating nurses through past decades. The tradition is marked by a shift from physician-trained to nurse-educated. The site of education has changed from the hospital to the classroom as a part of the larger setting of academe.

Although Florence Nightingale made valuable contributions to the health care of the British soldiers in the Crimean War, the American woman was looked upon as a disgrace while caring for strange men on a battlefield (Kalisch \& Kalisch, 2004). However, 
it was important that soldiers receive treatment. Gradually, attitudes shifted from highly patriarchical to acceptance that women could and should perform such services. Thus despite the negative perceptions about her status, Nightingale became the authority on the training of the nurse:

Nursing training was necessary to teach not only what is to be done, but how to do it....The nurse's eye and ear must be trained--smell and touch are her two right hands--and her taste is sometimes as necessary to the nurse as her head. (Kalisch \& Kalisch, p. 36)

In 1861 , in the United States, Dorothea Dix, whose experience had been in working with the mentally ill, was given the responsibility of organizing hospitals and nurses to care for sick and wounded soldiers. During that year, women were selected to take a short instructional course in caring for the soldiers taught by physicians and surgeons (Holder, 2003).

In the late 19th century physicians were recognized as being the authorities in teaching the nurse theory while a nurse superintendent and assistants taught the actual practice of nursing. Dolan (1968) noted that during this time the duties of the floor were more important than the classroom when patients were in need.

There was a lack of textbooks and training aids for nursing education during these early years. Many hospitals developed their own manuals detailing the practice of nursing. The first recognized nursing text was published in 1893 by Isabel Hampton, Nursing: Its Principles and Practice for Hospital and Private Use. It was used in the training of nurses for the next twenty years. Both class time and the presentation of theory were limited.

In 1884-1885, at one of the better institutions of the time, the Farrand Training School for Nurses at Harper Hospital in Detroit, the students has two annual series of lectures, which constituted the total theory content of the 24-month course....All lectures, including those on nursing, were given by physicians from 8:00 p.m. to 9:00 p.m. between October and March. For the remainder of the year, students provided service without the benefit of formal classes. (Kalisch \& Kalisch, 2004, p. 
In 1899 , Teachers College at Columbia University began teaching a course in hospital economy. Its purpose was to train graduate nurses to teach in nursing schools and to become superintendents in hospitals. The nurse spent several months in private duty for experience and then had additional training in the environment of the hospital and the various unique characteristics of the various institutions in that era. A certificate was granted at the end of the training period verifying the qualification for the nurse to supervise a training school or a hospital (Bullough \& Bullough, 1969; Kalisch \& Kalisch, 2004).

By 1909 there were 4359 general hospitals in the United States. There were 1006 of these hospitals which operated nurse training programs along with 90 mental institutions. Programs required a high school education and admitted applicants in good health between the ages of 18 and 35 years. The programs were three years in length and resembled the later hospital-based diploma nursing programs. Students as probationers would gradually begin working in the wards, applying the latest learning in the classroom to the patients on the wards. By the third month of training they would be working around six to seven hours daily. After the third month and after passing the necessary examinations, they were considered nursing students and were supervised by a "head nurse" who was a third-year nursing student (Kalisch \& Kalisch, 2004).

Around 1912 there were 10,000 nurses being trained each year, yet this was not enough to meet the needs of the population. Family members who needed medical attention were still being cared for in the home. Hospitals had the reputation of being poorly run and wasteful. There continued to be a growing need for additional nurses as medical advances created the need for additional hospitalizations. The need to recruit 
women into nursing careers was enhanced by the Women's Committee of the Council on National Defense. A survey conducted at that time in Cleveland, Ohio of 1139 high school graduates reported objections to the physically demanding work and the stressful environment. The committee found that less than one-fifth of the group was interested in nursing. The committee expressed the need for an overall recruitment effort and emphasized the need to decrease the required duty hours, make the duties more appealing and elevate the standards of how the nurses were being taught (Kalisch \& Kalisch, 2004).

From a desire to recruit better educated nurses, an experimental program was begun at Vassar College by providing post-graduate accelerated nursing courses in the summer of 1918. Students who had been educated as teachers as well as other professional positions were accepted. After taking classes 6 days per week with the necessary science, nutrition, and nursing skills, 418 completed the courses from the original 439 graduates who were accepted. After completing the summer program students were employed in hospitals from New York to Michigan. Building from this successful approach to learning, five other colleges began training programs (Kalisch \& Kalisch, 2004).

A committee was formed in 1919 to look at the education of public health nurses. While examining public health education, the group realized that it was not just education of public health nurses but all nurses that was in question. The Committee for the Study of Nursing Education funded by the Rockefeller Foundation and composed of physicians, nurses, public health officials, and members of the public extensively surveyed twentythree hospital training schools in the United States, representing the diverse nature of the educational institutions. The publication that emerged from the committee's findings was called the Goldmark Report after the secretary of the committee, Josephine Goldmark. Ms. Goldmark was a leading nurse educator who gathered extensive information from surveys 
of nurse educators related to conditions of the schools of the time. This provided the direction for basic undergraduate training for all nurses. The report also recommended that nursing education lead to an advanced degree consistent with other professions. The committee cited many factors contributing to the poor quality of nurses, including the lack of correlation between practice and theory and the lack of well-qualified teachers. The necessity to provide a liberal arts education as opposed to just providing training was seen as a fundamental need (Bullough \& Bullough, 1969).

In 1909, the University of Minnesota was the first college to begin a nursing program as part of the institution. The university offered a 3-year diploma under the college of medicine; this was a unique step for nursing education. Prior to this time colleges had nursing programs but they were still housed in the hospital and were not considered to be part of the college. By 1916 there were 16 nursing schools that were considered part of a public or private college. Students after four or five years would receive a nursing diploma as well as a bachelor's of science degree. By 1926 there were 25 schools of nursing, but enrollment was less than 400 students, emphasizing the popularity of the hospital-based programs over the college. The Yale School of Nursing opened in 1924 as the first separate and independent school of nursing having its own dean and accompanying budget. Its success led to a million dollar grant for continued operations of the 28-month program leading to a degree in nursing. The method of teaching included the application of a variety of theories and previously learned knowledge, providing an opportunity for the student to begin studying all aspects of the fields of medicine and the nursing care of the patient. However, within five years requirements were changed to require a bachelor's in arts, science, or philosophy for admission to the program and at the end of thirty months a Master's degree was awarded (Kalisch \& Kalisch, 2004). 
Despite the advances there were plenty of people in medicine and the lay public who felt that nurses were receiving too much education in the colleges and the training given at the bedside was sufficient. Hospital-based training continued to be the mode of training of nurses and nursing schools in universities like Yale were not growing to match the need. This anti-intellectual trend mirrored much of the thinking in the wider United States society (cf. Hofstadter, 1994).

By 1927 there were 2286 programs of nursing while medical programs were closing. The Flexner Report in 1910 altered the medical education standards and several schools offering medical degrees closed. In 1920 there were 3000 medical graduates whereas in nursing there were 7700 graduates (Berger \& Williams, 1999; Kalisch \& Kalisch, 2004).

Nursing practice and nursing education were affected by a report released in 1948 , Nursing for the Future, by Esther Brown (cited in Stewart \& Austin, 1962), a researcher at the Russell Sage Foundation primarily interested in the social sciences, including economic and social conditions. Dr. Brown, who was not a nurse, reported that nursing education was not financially assisted by society as was the case for teacher training. The recruitment of nurses was hampered by the conditions that were present in the education process and the profession itself. She supported the movement of nursing programs from the hospitals to universities and colleges to equal that of medical education. She noted that conditions in nursing schools were so poor that each school should be evaluated for enrollment and the success of its graduates. She suggested that society should assist in the financial responsibility of a comprehensive evaluation of all nursing programs.

Like other professions, it (nursing) was finding in colleges the proper intellectual climate for the preparation of the professional worker. Nurse educators with vision were strongly impressed with the need for better teaching and for better resources 
in the libraries, laboratories, and other physical facilities for education available in colleges and universities. (Kalisch \& Kalisch, 2004, p. 336)

From the Brown Report came a realization among nurse leaders that accreditation of programs could result in the improvement of conditions in schools of nursing. The National Committee for the Improvement of Nursing Services was formed from the committee organized to implement the Brown Report. A survey was developed to analyze individual nursing programs, using a scale to collect data related to program criteria. Evaluation of faculty, curriculum, library resources, graduate success on licensing exams, overall conditions regarding student welfare, as well as financial conditions were included. Schools were ranked based upon their self reporting information. A published report, Nursing Schools at Mid-century (cited in Stewart \& Austin, 1962), listed the names of the schools and their rankings. Schools ranked in the Group III category, the lowest and considered the poorest conditions, surprisingly had 13,779 students enrolled in their programs of nursing. Programs in the bottom tier were upset as were the programs that were not considered in the publication since they were not surveyed. Some administrators were concerned that the personnel returning the surveys may not have been informed as to the use of the survey. A national outcry came from the organization representing hospitalbased schools and the criticism of the process was seen as a measure to move all nursing education programs to a college setting.

Federal funding for nursing education was a struggle. The national organization formed to represent hospital-based nursing programs, the National Organization for Hospital-Based Schools of Nursing (NOHSN), publicly criticized any legislation aimed at providing additional funding for college programs. Their workforce continued to consist of student nurses who provided cheap labor to the hospital. Students paid tuition and hospitals 
prospered. However, as the reality of federal funding for nursing education emerged, additional federal dollars was seen as providing additional benefits and money for the operation of the hospital and the administrators were supportive of such an opportunity (Dolan, 1968).

As nursing education moved forward to the 1950 s, there were obstacles that prevented the growth of baccalaureate programs despite the need for nurses. Competition intensified between baccalaureate programs (mainly those that were specifically on college campuses) versus those that were hospital-based while providing some additional science and general education courses. The National League for Nursing projected an increasing need for college-educated nurses to be at least one-third of all nursing graduates. However, in 1962 only $14 \%$ of the nursing students were graduating from collegiate programs. The increase in baccalaureate programs and expansion of nursing programs created a shortfall in the numbers of students completing master's programs and those who were acquiring the preparation to teach the nursing students. "Added to the needs of the schools were the demands for administrative and supervisory personnel of approximately 6000 hospitals and 7800 health agencies and boards of education. It could readily be seen that the nursing administrator had to compete for a scarce supply of well-qualified faculty" (Kalisch \& Kalisch, 2004, p. 390).

As nursing continued to evolve in the collegiate setting and away from the hospital sponsored programs, a major nursing shortage was looming in the early 1960s. It was estimated that 130,000 more nurses would be needed for the profession by 1970 . To reach that goal additional nursing programs would be needed and current nursing programs would need to expand. Consultants appointed by the U.S. Public Health Service Surgeon General reported the need for 3000 nurses educated at the master or doctoral level to teach 
(Kozier, Erb, Berman, \& Snyder, 2004).

To help meet the need for additional nurses the associate-degree nursing program was designed to educate the nurse student on a technical level. This would reduce the time necessary to educate the nurses. Mildred Montag, in her doctoral thesis (cited in Kalisch \& Kalisch, 2004), developed the role of the associate degree nurse as a new prospective healthcare worker who would complete the educational requirements in a junior college setting. The students enrolled completed courses in liberal arts and general education as well as the ability to enter into the job market immediately upon graduation from the respective programs. Within a period of 10 years enrollment in associate-degree nursing programs had gone from 1132 to 15,338 (Deloughery, 1977; Kalisch \& Kalisch, 2004). The addition of a new program to educate a new healthcare worker required additional qualified faculty. This requirement led to direct competition between the baccalaureate and the associate degree programs for staffing a nursing program.

Today the nursing shortage continues as does the demand for master's prepared nurses to teach in all types of nursing programs. The following information details selected research articles which provide insight on the field of nursing.

\section{Research Studies in Nursing Education}

Through the work of researchers comes additional information that provides insight into the development, the recruitment, the retention, and the satisfaction of the nursing faculty member. The greatest amount of research dealing with nursing consists of clinical practice and the work of the nurse in the clinical setting. Although not as voluminous, there is some data that has been collected over the years on nursing education, providing insight into the role of the nurse in academe.

Baurhaus, Staiger, and Auerbach (2000) identified and assessed key indicators of 
changes in the age distribution and the total supply of RNs. The authors projected the future age distribution and the total RN workforce as a means of identifying the gravity of the nursing shortage through the year 2020. Data from the United States Bureau of the Census Current Population Surveys were part of a retrospective cohort analysis of employment trends of recent $\mathrm{RN}$ cohort groups. Workforce trends were used to forecast long-term age and employment of RNs. The participants were employed RNs in the United States in the age range of 23-64 years of age $(N=60,386)$. An analysis of variance (ANOVA) was used to estimate the parameters of the equation, identifying the number of full-time RNs as compared to the populations of RNs. The results indicated that in the next 10 years, the average age of RNs is forecasted to be 45.4 years, an increase of 3.5 years over the current age, Around the year 2020, the RN workforce is predicted to be about the same size in number as it is today, which is a $20 \%$ decline below projected $\mathrm{RN}$ workforce requirements. The researchers concluded that the major reason for the aging population of the $\mathrm{RN}$ workforce was the alternative career choices available to young women today. They also indicated that a critical time will be when the first of the baby boomers, numbering 78 million, begin to retire in 2010 and enroll in Medicare sponsored programs, leading to an additional need.

Another concern from administrators of nursing programs is the trend of graduate programs to focus only on advanced practice nursing tracks to meet the demand of those caring for the growing elderly population. This number will increase as the baby boomers reach the age for Medicare. Fewer nurses are interested in positions in nursing education; furthermore, the demand for nurse practitioners puts additional demands for staffing graduate nurse education programs (Beurhaus et al., 1994; Brendtro \& Hegge, 2000; Craine, 2000). 
In a descriptive study, Ryan and Irvine (1994) examined current and projected faculty profiles, policies related to retirement, and projected replacements for schools of nursing for the next 15 years. Using a revised Nursing Faculty Profile used in previous research, the reviewers asked six nationally known nurse educators/administrators to review and establish face validity. The questionnaire consisted of four sections: (a) demographic characteristics of the institutions and faculty, (b) characteristics of policies and procedures related to retirement; (c) current and anticipated availability of qualified faculty by specialty area; (d) and for schools with graduate programs, projected numbers of graduate students. The questionnaires were mailed to all baccalaureate and higher degree programs accredited by the National League for Nursing. There were 514 questionnaires mailed to the schools. The final sample for analysis was 296 , a $58 \%$ return rate. Demographically $49.1 \%$ of the schools returning questionnaires were public institutions, $48.4 \%$ were private, and $2.5 \%$ were secular. The mean number of students in each program was baccalaureate $(M=274)$, master's $(M=98)$, and doctoral $(M=34)$. Overall $96 \%$ of the returnees were female, with a peak age range from 41-50 (43\%). There was a younger but somewhat equal distribution of faculty 40 years or less (28\%) and those 51 years to 60 years (24\%). The results demonstrated that $76.1 \%$ of the programs did not have a mandatory retirement age, and $63 \%$ did not have incentives for early retirement. Although almost half of the institutions offered systematic retirement planning for staff, almost $80 \%$ stated they were not currently reviewing any type of faculty retirement plan.

Projected retirement plans for the next 15 years were obtained from actual numbers recorded from the survey of projected and actual resignations for the 1992-2006 period. The estimate totaled an average of six retirements per nursing unit in the next 15 years. Voluntary resignations were not anticipated to be more than an average of two per nursing 
unit or one-third of the current faculty work force. Administrators in the survey answered that they had not had difficulty in recruiting full-time faculty but they had not seen an increase in qualified applicants. Of the administrators responding, $57 \%$ noted a decline in qualified applicants. Sixty percent admitted to having difficulty in getting qualified applicants to accept positions. Administrators did not expect a decrease in the number of faculty but many projected an increase in the need for hiring full-time faculty (Ryan \& Irvine, 1994).

Specific areas of clinical expertise were noted to contribute to the problem. Onefifth of the respondents from undergraduate programs admitted to having difficulty in hiring qualified faculty in the medical-surgical area while $50 \%$ of the administrators reported that it was hard to find faculty in the specialties of pediatrics and maternity/family nursing. Heads of graduate programs did not share the same concern about finding applicants for positions in the specialty areas. Ryan and Irvine (1994) projected graduates with advanced degrees over the next five years from the study would be 4,500 master's students in various clinical areas with over half in adult health. Numbers for pediatric, community health, and psychiatric/mental health graduates were predicted to be about 850 students per area of specialty. In the area of role specialization at the master's level, preparation in administration $(1,350)$ ranked higher than teacher education (less than 800 graduates). Estimates predicted that over 8,350 graduates will be entering the job market. Graduates from doctoral programs were estimated at 450 in five years with 14 programs reporting. Administration and teacher education estimates were 100 graduates each over the next five years.

The researchers noted that with private and public educational systems grappling with financial concerns that have an impact on availability of economic neither state nor 
institutional resources may be forthcoming to support current replacement, or additional faculty needs. Anticipated retirements may lead to a significant turnover within the nursing professorate. Competitive salaries and flexible work schedules in nursing practice areas are luring nurses with advanced degrees away from academe. Ryan and Irvine (1994) used the term "bipolar professorate" to characterize the senior and junior faculty in nursing education. The study suggested that there would be a shortfall in doctorally prepared nurse graduates since estimated replacements would total only about half of expected losses.

From the previous study, the aging $\mathrm{RN}$ workforce as well as the decline in finding qualified faculty depicts a crisis in nursing education. As the demands for nurses rise and the numbers of nurse educators leave the profession through retirement, higher education must be able to provide well-qualified faculty to meet the challenge. Recruitment and retention will be of utmost importance.

The next section addresses the career choice of those in nursing, with subsections on faculty attraction to fields other than nursing, such as the community college setting and recruitment in nursing education. There has been some research carried out particularly for nurses, yet much of the research is limited to earlier periods of nursing shortages.

\section{Recruiting Nursing Faculty}

Boughn (1999) conducted a qualitative research study based on grounded theory to determine the needs and expectations of females interested in nursing. Her sample consisted of 16 participants between the ages of 18 and 40 enrolled in a baccalaureate nursing program at a state college. During an interview with students demographic data were collected concerning age, ethnicity, marital status, family group, and the reasons for their career choice. Research questions included, "Tell me your earliest memory when you thought about becoming a nurse?" and "What is your view of an ideal nurse?" Questions 
used during the interview were from a previous parallel study and according to the researcher, achieved highly correlated results. Experts in the nursing education department reviewed the questions for validity.

Data were subjected to a constant comparison process and coding. Boughn (1999) found power and empowerment of self and others as themes from the interviews. Financial reward and job security were also responses often present. Another theme identified was the need to be needed, the desire to care for others. Responses differed in relation to the level of the nursing student. Freshmen connected with the "power of nursing skill and knowledge" while the sophomore level student, according to Boughn, was concerned with personal power. Leadership, delegation, and supervision were important to the students. The junior level student was thought to be more sophisticated and had more of a desire to take control, to have autonomy and power, and to look at the business side of nursing. The senior level student demonstrated what the researcher called "protective feelings" for the profession.

Research on career choice of nursing by males has offered some understanding of the slow movement into nursing. Men are entering the profession in larger numbers; however they still constitute a small minority. The image of nursing as being caring and compassionate may be barriers to career choice. Some members of society may not be able to adjust to the role of the male demonstrating caring behaviors. Cultural constraints and the normal division of labor cause one to rethink the concept of masculinity. Men interested in the role that is female-dominated may feel that it lessens their masculine role.

Okrainec (1994) examined the perceptions of nursing education held by male nursing students in nursing programs in Alberta, Canada. He noted that there had been limited research in nursing in the area of males in nursing. In his study he posed six 
questions: (a) What are the demographic characteristics of male students, (b) What interests do male nursing students express in nursing education, (c) What attitudes do male nursing students hold toward nursing as a career, (d) What is the level of satisfaction of male nursing students with nursing education, (e) What image of themselves as nurses do male nursing students hold, and (f) Do male nursing students differ from a comparative sample of female student in any of the above characteristics. A 72-item survey was sent with a cover letter to a target population of 328 nursing students (164 male and 164 female) at 13 schools in Alberta, Canada after it was pilot tested by nursing students and faculty at one school in Edmonton, Alberta, Canada. An equal number of female students as male students were selected within each year and type of program by matching random numbers of females as they appeared on class rosters. The author did not state measurement of internal reliability of the survey. Completed questionnaires were returned over a six-week period of which 117 males (71\%) and 121 females (73.7\%) were returned. The responses to the surveys were analyzed using descriptive and correlation methods.

Okrainec (1994) concluded that the men in the study were older than women with a mean age of 28.2 for males and 24.6 for females. Males indicated stronger postsecondary educational backgrounds, yet tended to have less favorable high school grade point averages than the females. A greater percentage of women (54.5\%) compared to men (46.2\%) indicated their high school grades were above average. More men (47.9\%) than women (38.3\%) had completed postsecondary education programs prior to entering into nursing school. Over $70 \%$ of the men did not decide on nursing as a career until past the age of 20 . Women (43\%) had become interested in nursing at the age of 16 years. More men than women had relatives or friends that influenced their decision to become a nurse. For men, $71.3 \%$ and for women $67.5 \%$ had relatives or friends in nursing. Both male and 
female respondents indicated they entered nursing for similar reasons, the opportunity to help people; for males (87.2\%) and females (89.3\%). The next two major influential factors were employment opportunities and income. More men than women responded that they would recommend nursing as a career to other men: $(92.3 \%)$ men, and women (89.2\%). In terms of longevity, more men (89.6\%) expected to be working full-time in nursing 10 years from graduation whereas only $63.9 \%$ of the women expected to do so.

Both male and female respondents attributed a natural aptitude for nursing to women than to men. About $30 \%$ of both sexes thought that the female nurses were superior to the male nurses. When caring attitude was considered, $79.4 \%$ of the males and $73.3 \%$ of the females responded that caring attitudes were equal in both genders. From Okrainec's (1994) study, the number of males who would recommend nursing to other males reflected a change in societal expectations. The interest and satisfaction in their education and the anticipation in their future career in nursing provided the sense that nursing for males and females is a viable career choice. Both men and women are valued for the role they play in nursing. The number of research articles on men in nursing is limited, and the role and expectations of a male nurse faculty member seems even more difficult to determine. Research is very limited in the area of a male nurse faculty member in academe.

Research into recruitment of faculty members in general lends a wealth of knowledge concerning characteristics of the candidate. From the literature, colleges have seen increased difficulty in recruiting faculty to college positions.

\section{Attracting Experienced Faculty (Non-nursing)}

The declining number of nurse educators and the forecast of further shortages in the future make it imperative to look at recruitment of additional faculty. There is little empirical research on recruitment of nursing faculty. Likewise community college officials 
are faced with many of the same concerns as nursing. Large numbers of community colleges proliferated in the 1960s and many of the faculty hired at the time still remain at the institution. Participants in an American Association of Colleges of Nursing ([AACN], 2003) study were full-time teaching faculty at least 55 years of age and currently teaching in academic areas of general education/transfer business, or developmental education at institutions listed in the 1996-1997 American Association of Community Colleges directory. All regions of the country and all sizes of institutions were considered in the study. A proportionate random sample was determined using Kalton's formula for sample size determination. A total of 302 institutions were identified and 1,154 questionnaires were mailed to all of the representative institutions.

The authors (AACN, 2003) developed two questionnaires: one was completed by the chief academic officer (CAO) and the other by faculty members. The faculty member questionnaire collected data concerning numbers of years taught, disciplinary areas where taught and, in addition to estimated retirement dates, concerns related to retirement and factors perceived to affect retirement. No internal measures of reliability were reported. Descriptive statistics were used to analyze the data. The response rate for the CAO questionnaire was $16 \%$ or $48 \mathrm{CEOs}$ out of 302 possible subjects. The return rate for faculty questionnaires was $42 \%$ with 332 faculty responding out of a possible 1,154 total questionnaires mailed. More respondents representing the North Central and Southern accrediting areas returned questionnaires. The findings indicated that the disciplines of social sciences (33\%), and arts and humanities (29\%) had the highest proportion of faculty 55 years and older. Twenty-five percent of current full-time faculty members in all disciplines were 55 years of age or older. The smallest percentage of faculty members nearing retirement age, using 65 years as the retirement age, were the disciplines 
representing developmental education (23\%) and biological sciences (20\%).

Ninety-four percent of the faculty members in the study stated they would retire within the next 10 years. Faculty members with definite plans in place estimated that they would retire 1.2 years earlier than the faculty without definite retirement plans. Nationally, according to the AACN (2003), the average number of full-time faculty predicted to retire in the next 10 years was $84 \%$ or 25,850 to 30,040 .

From the questionnaire, subjects rated financial resources, personal health, and access to health insurance as the three top issues affecting their retirement decisions (no values given). Additional positive responses affecting retirement age included the opportunity for a career change as well as the development of additional sources of income in retirement. Over one-third of the institutions that responded had an early retirement plan in place. Only $11 \%$ of the institutions had a phased out plan in place which reduction of faculty member's workload each year until fully retired with a corresponding reduction in benefits or salary. Nine percent of the colleges reported that some form of retirement plan was under consideration (AACN, 2003).

Faculty members also were asked to rate 21 characteristics and skills new faculty replacements should possess. The five most important skills as rated by the faculty were: (a) ability to plan and implement effective class sessions, (b) ability to develop a fair grading system, (c) ability to establish clear goals and objectives, (d) ability to lead classroom discussions, and (e) competence as a lecturer (no values given). The researchers identified a need to develop a training program to assist with recruitment and hiring of the best-qualified applicants. New faculty should be "groomed" for positions by internship and scholarship programs (AACN, 2003).

Fifty-one of the CAOs reported that they anticipated having difficulty recruiting 
educationally prepared faculty members. Replacement of computer science faculty was the greatest concern, followed by physics, biology, chemistry and foreign language faculty. This study (AACN, 2003) identified an emerging problem in replacement of faculty due to retirement and the subsequent need for recruitment of new members to the profession.

Additional studies exploring recruiter and applicant attraction have been published. Winter and several associates examined students currently enrolled in graduate programs to explore the effect of the recruiter, the advertisement, the location of the job, and/or gender in the simulation of the recruitment process.

Winter (1996) determined whether or not organizational characteristics and job attributes described in recruitment advertisements attract applicants for community college business faculty positions. The investigator's own recruitment-as-job-theory was applied to evaluate the recruitment of community college faculty. Two hypotheses were formulated: there will be no difference in applicant reactions to advertisements carried according to instructional program context (academic transfer, career education, and compensatory education), and there will be no difference in applicant reactions to advertisement which varied according to job attribute content (intrinsic, extrinsic, and work context). The design was a 3 (times) 3, completely crossed, fixed factor analysis of variance (ANOVA). The independent variables were, instructional program (academic transfer, career education, and compensatory education), and job attributes (intrinsic, extrinsic, and work context). The dependent variable was an additive composite rating of applicant reactions to a community college business faculty recruitment advertisement. A five-point Likert-type scale was used, ( 5 being more favorable than 1$)$. The characteristics included: (a) overall attractiveness of the job, (b) likelihood of applying for the job, (c) likelihood of accepting an interview for the job, and (d) likelihood of accepting the job if offered. 
The participants were experienced male and female business professionals pursuing MBA degrees $(N=162)$. They were asked to role-play applicants reacting to faculty recruitment advertisements similar to those placed by community college administrators. To comply with the Winter (1996) Model, the applicant characteristics were held constant: (a) level of applicant experience, (b) nature of job experience (business), (c) degree credential (MBA), (d) position desired (business faculty), and (e) level of postsecondary position sought (community college). Subjects were selected at random from a pool of experienced business professionals enrolled in graduate classes at a large southeastern university and randomly assigned treatment conditions. The independent variables were manipulated via nine formal recruitment advertisements. Content of the advertisements consisted of: (a) general information, (b) applications procedures, (c) instructional program description (manipulated to be academic transfer, career education, and compensatory education [developmental]), and (d) job attribute descriptions (manipulated by rating extrinsic, intrinsic and work context).

Results of the study supported the emphasis by recruiters of the academic transfer mission. The career education programs and the compensatory programs were viewed less favorably when recruiting applicants from MBA programs at four-year institutions. Job attributes emphasized in recruitment advertisements may be either intrinsic, extrinsic, or work context attributes, provided the pool is composed of both men and women. A statistically significant main effect for instructional programming was identified $(F=$ $149.62, \mathrm{p}<.0001)$. Compensatory programs were rated less favorably, career educational programs were rated more favorably than compensatory programs, and transfer academic programs were rated the most favorably (Winter, 1996).

Winter and Kjorlein (2000a) empirically examined potential applicant reactions to 
position announcements for business faculty vacancies at a community college and identified predictors of applicant decisions, such as decisions to apply for a job. The method was a simulation of potential applicant reactions to position announcements. The correlation design was a manipulation of a recruitment exercise intended to assess applicant's reactions to two experimentally manipulated variables: recruiter background and job relocation. The participants were experienced male and female business professionals pursuing Master's in Business Administration (MBA) degrees chosen at random from a pool of experienced businessman enrolled in the MBA program $(N=176)$. Manipulation of the information was accomplished by a detailed advertisement with a job location requiring instate or out-of-state relocation. The recruiter's background was similar to the participants (experienced business professional, entered community college teaching from a private sector, and having a Master's in Business).

Each applicant completed a biographical sheet with information such as age, gender, race, marital status, number of children, and current job satisfaction, then assumed the role of a potential applicant and read a simulated faculty position announcement. The subjects also completed a questionnaire using a five-point Likert-type scale to assess the job vacancy described. The stepwise multiple regression procedure revealed the most significant predictor was the applicant's current job satisfaction (increment in $R^{2}=.484$ ), when applicant rating of the job increased, the current job satisfaction decreased. The second predictor was applicant rating; rating of the job increased as the percentage of the household income earned by the spouse decreased. No other personal characteristics were statistically significant. The third predictor, the rating of the business faculty job, was more favorable when the background of the recruiter was similar to that of the applicant and the job did not require relocation. The four significant predictors accounted for $52.4 \%$ of the 
variance in the applicant rating of the business position (Winter \& Kjorlein, 2000a).

Winter and Kjorlein (2000b) built upon their previous research and looked at faculty recruitment and its effect on job mobility, recruiter similarity and dissimilarity, and the gender of the applicant. The study design was a $2 \times 2 \times 2$ completely crossed fixed-factor ANOVA. The independent variables were recruiter characteristic, job vacancy characteristic and the applicant characteristic. There were 136 participants with males $(n=$ $68)$ and $(n=68)$ females, chosen from business professionals completing an MBA degree. Participants were selected at random and assigned to one of eight treatment conditions. Each participant read and evaluated one of four business faculty position announcements. The group participates in a simulation by role-playing as applicants for a community college business faculty position. The participants performed three tasks: (a) completion of a biographical data form, (b) review of a simulated faculty position announcement, and (c) completion of a position announcement evaluation. The biographical data instrument was a 7-point Likert-type scale $(1=$ not at all willing, $7=$ very willing $)$.

The instrument used to evaluate the position announcement contained four items: (a) "How would you rate the overall attractiveness of the teaching job described?" (b) "How likely would you apply for the teaching job?"(c) "If offered, how likely would you accept an interview for the teaching job described?" and (d) "If offered, how likely would you be to accept the teaching job described?" Each item was evaluated using a 5-point Likert-type scale with 5 being most favorable and 1 being less favorable. Both male and female applicants preferred jobs described be a recruiter who shared similar backgrounds with the applicants $F(1,128)=11.97, p<.001$. Recruiter background after omega-squared indicated that the recruiter similarity/dissimilarity accounted for $7.4 \%$ of the variance in the applicants rating of a business faculty position. The applicants also preferred jobs that 
did not require relocation $F(1,128)=5.16, p<.05$ (Winter \& Kjorlein, 2000b).

Winter and Munoz (2001) conducted an exploratory study on the association between personal characteristics and job ratings of applicants for a business faculty position in a community college setting. The authors cited the lack of research in the practice of recruitment in the community college setting. The need to recruit college faculty members to replace the aging baby boomers nearing retirement was an important reason for the research. The study was a recruitment simulation involving correlational analysis using ordinary least squares stepwise multiple regression. The participants were experienced business professionals enrolled in MBA classes at a major university. Their task was to role-play the part of job applicants for a community college setting, as in other Winter et al. studies. The independent variables were applicant's demographic characteristics and applicant's personal satisfaction with their current position. Demographic variables included: (a) marital status, (b) working status, (c) age, (d) number of dependents, (e) years lived in the area, (f) hours worked per week, (g) job tenure, (h) total years of work experience, and (i) years of business experience. The dependent variable came from the short form of the Minnesota Satisfaction Questionnaire designed to measure job satisfaction of participants in relation to 20 intrinsic and extrinsic job features. Coefficient alphas for the intrinsic and extrinsic composite scales were 0.87 and 0.77 , respectively.

Winter and Munoz (2001) conducted a pilot study to assess the reliability of the job satisfaction scales and the dependent variable composite score. The Cronbach's alpha for the job satisfaction measures was 0.89 . On pilot data the participants were selected at random from those who volunteered to participate. There were 194 subjects in the sample. The study required the participants to complete a biographical data form and a job 
satisfaction instrument and to rate a business faculty job vacancy detailed in a simulated recruitment advertisement, similar to those used by community college recruiters. Results indicate that marital status $(r=0.23)$ was the independent variable that correlated the highest in explaining the variance in applicants' ratings of the faculty position. There was no significant correlation between the dependent variables and gender, race, business experience, job tenure, and job satisfaction. When the two variables marital status and hours worked per week were entered into the stepwise regression equation, marital status was the independent variable that made the highest contribution to explaining variance in applicants' job rating (5\%). There was a negative correlation between marital status and applicant's job rating; when participants were not married their attraction to the job increased. Hours worked per week explained $3 \%$ of the variance; the more hours worked per week the attraction to the job increased.

Each of the preceding studies looked at the simulation of an applicant's attraction to a job in the community college setting. The research indicated that the background of the recruiter, the location of the job, the marital status, and the hours worked per week in the job were important for recruiting applicants. Examined next is research dealing with attraction to nursing in two studies of experienced nurse educators (Froman, 1996) and personality characteristics of nursing faculty compared to other academic women (LiaHoagberg, 1982).

\section{Attracting Experienced Nursing Faculty}

Froman (1996) conducted a study using survey research to determine ratings of importance both for fixed and alterable characteristics of nursing faculty positions. Two dimensions of importance were considered to be distinct for the survey: (a) importance in initially attracting potential candidates and (b) the importance in determining the 
acceptance of the job by the expert nurse. Securing a seasoned nurse educator or researcher with experience and recognition in the field is a challenging task for schools of nursing. Two dimensions must be examined, those that are alterable (salaries, titles, and teaching loads) and those that are fixed (geographic location of an institution, its proximity to a city and prestige of a university). Knowledge of the relative importance of these elements might assist in negotiating job descriptions with administration before the job is posted. The researchers developed a questionnaire with 16 job characteristics with two self-report options ranging from 1 to 5 response options (1, initial interest-not very important to 5 , initial interest-very important). The second response scale was anchored by the descriptors (1, accepting an offer-not important to 5, accepting an offer-very important). Respondents were instructed to rate the importance and interest to the subject in applying for a university position. A pilot test was conducted with two samples of nursing faculty and a convenience sample of 30 graduate students in a master's preparation program.

Eight hundred questionnaires were mailed to members of the Academy of Nursing from a 1992 mailing list with 295 respondents returning questionnaires (37\% response rate). Ninety percent of the respondents were women with an average age of 52.5 years. Two thirds (68\%) were currently employed at a college or university and were engaged in the functional areas of administration, consultation, teaching research, direct clinical practice, or a combination of the four areas (Froman, 1996). Data were analyzed using descriptive and correlational methods. The top rated items for stimulating interest in a position and determining acceptance with an average mean rating greater than 4.3 on a 5.0 scale were: (a) research support $(M=4.36)$, (b) salary $(M=4.34)$, (c) autonomy of the nursing unit $(M=4.33)$, and (d) potential for professional collaboration $(M=4.32)$. Proximity to city, proximity to health center, and job title were the least important. To 
determine if university or non- university-based nurses differed in their ratings of the characteristics, a pair of discriminate function analyses was conducted using the rated 16caharacteristics both for importance of interest and importance for accepting an offer. Each showed that approximately $10 \%$ of group membership was accounted for by the set of the 16 item ratings taken together. Classification functions showed that the "autonomy of the nursing unit" and the "research support available" items were rated higher and more important to those in the academic group whereas salary and access to clinical facilities were more important to non-university nurses.

Froman (1996) concluded that to recruit a university-based nurse, it was important to highlight support for research when advertising, along with administrative structure and autonomy at the school. When recruiting non-university candidates, advertisement of salary and clinical practice potential would be important. Although the study was national in scope, the sample of nurses holding membership in the Fellows of the American Academy of Nursing (FAAN), an elite group of nursing professionals whose mission is to advance nursing science, represented an important demographic; however, including other experienced nurses in education would also be important.

Witney (1990) investigated the relationships among several measures of selfconcept and several leadership behavioral variables with a sample of nurse educators from 23 National League for Nursing (NLN) accredited baccalaureate nursing programs in a Midwestern state. Self-concept measures were (a) self-control, (b) self-significance, and (c) self-competence. Leadership behavior variables included: (a) meeting organizational needs, (b) managing resources, (c) leadership competence, (d) task accomplishment, and (e) communications. Research questions were (a) What is the relationship between two groups of variables: a self concept variable set and a leadership variable set? (b) What is 
the relationship between the self-concept variable and each leadership variable? and (c) What is the relationship between each self-concept variable and each leadership variable? Nurse educators who returned questionnaires $(N=85)$ ranged between the ages of 29-65 with the mean 43.59 years. The respondents had been in nursing for a mean of 20 years. Of administrative experience $53.8 \%$ had $0-2$ years of work experience in administration. Instrumentation consisted of a Personal Data Questionnaire to gather information concerning age, years of professional nursing experience, current teaching level (undergraduate or graduate), and student enrollment in program. These elements were used in assessing the needs of and interrelationships of working groups and to create an organizational climate conducive to effective change. There were four measures: (a) Element B-Behavior measured the respondent's behavior toward others in general and their behavior toward the respondent, (b) Element F-Feelings measured an individual's feelings toward others and reciprocally that person's perceptions of how others feel toward the respondent, (c) Element R-Relationships provides an opportunity for in-depth exploration of the interpersonal relationships between individuals, and (d) Element S- SelfConcept was the only element chosen for the research to assess how the respondent felt about self. The researcher reported a high degree of internal consistency, but no other information. Each element consisted of 54 items answered on a six-point scale ranging from "Definitely not true" to "Especially true." Each item was answered twice, once describing the way things were perceived and once indicating how the respondent would like them to be (Witney, 1990).

Respondents also completed by The Nurse Practitioner Leadership Questionnaire (NLPQ), an instrument with five dimensions to measure self-reported leadership behaviors in nurse practitioners. It contained 37 items with coefficient alphas for internal consistency 
of the five dimensions ranging from .60 to .79 . The five-dimensions measuring selfreported behaviors in nurse practitioners. The five dimensions were: (a) meeting organization needs, (b) managing resources, (c) leadership competence, (d) task accomplishment, and (e) communications. Witney (1990) analyzed the data using canonical correlation, Pearson product moment correlation, and regression analysis. A canonical analysis pertaining to the relationship between two or more dependent and two or more independent variables was computed to determine the relationship between selfconcept and leadership variables. Pearson product-moment correlation coefficients were calculated to determine the relationship between each self-concept and leadership pair. A $t$ test was utilized to test the significance between pairs of correlations (Witney, 1990).

Results of the analyses indicated that the relationship between self-concept and leadership behavior was not statistically significant. Two leadership behaviors were significant relative to their relationship with self-competence, communications, and task accomplishment (self concept variable set and managing resources were statistically significant, $\left.R^{2}=.11, F(3,76)=3.25, p=.01\right)$. As a nurse's self significance is enhanced, leader behaviors should improve and increase, specifically in the areas of managing resources and communications. There were significant relationships between selfcompetence and four of the five leadership behavior variables. These were self-competence and managing resources, self-competence and leadership competence, self-competence and task accomplishment, and self-competence and communication. Communications emerged as a key leadership behavior variable in all of the significant relationships. Implications for recruitment of nursing faculty include the necessity to seek those candidates who express themselves well, who have had leadership roles, and who are willing to search for opportunities for faculty to develop further leadership and communication development. 
Developing leaders is as important as seeking those that come to the educational setting with leadership experience.

\section{Retention of Nursing Faculty}

Using knowledge obtained from research on the retention of nursing faculty and recruitment in other disciplines can assist faculty and administrators in developing strategies to recruit quality nursing faculty. This information can play an important part in the employment of quality candidates for nurse educator roles. Nevertheless, faculty who are employed must be retained and research on this problem diverges from that on recruitment. This section addresses these issues, including research on job satisfaction, role conflict and role strain, burnout, and programs to retain new faculty members.

\section{Job Satisfaction Among Nursing Educators}

Nurse educators who are not happy in their position have left academe for the clinical world to avoid the high levels of stress (Seller \& Pearson, 1984). Retaining quality faculty is also important for a nursing program. Professional nurses often want the opportunity to give care as well as teach. The climate of the clinical world is rapidly changing and as the nursing shortage deepens, more pressure will be applied to faculty to assist in the care of the patients in spite of the educator role. Job satisfaction can be a major factor in determining whether nursing faculty will endure the stresses of their job or seek other professional opportunities. As in the previous section, there are often strong parallels between community college and nursing faculty.

Milosheff (1990) examined job satisfaction in community college faculty by sampling 703 full-time faculty members from 35 institutions with two years or more teaching experience. The subjects completed a survey inquiring about their backgrounds, interests, professional activities, perceptions of students and the institution. Questions for 
research were (a) What is the relationship of personal and demographic characteristics on job satisfaction? (b) What is the relationship of professional activities on job satisfaction? (c) What is the relationship of perceptions and relations with students with students on job satisfaction? and (d) What is the relationship of institutional environment and departmental environment on job satisfaction? Independent variables included: (a) personal and demographic characteristics (educational degree attainment and gender), (b) professional activities, (c) perceptions of and relationships with students, (d) institutional environment, and (e) departmental environment. The dependent variable was job satisfaction. After entering both independent and dependent variables into a regression equation, it was found that none of the variables were highly correlated with each other. Additional analysis was conducted looking at job satisfaction based upon gender and department. Five variables-(a) influence at the college, (b) perceptions of the students, (c) adverse financial condition of the institution, (d) intellectual quality of the institution, and (e) department colleagues-were significant at the .005 level. A sixth variable, time spent on in-school activities, was significant at the .05 level. The most significant variable was the perception of students. This had twice the beta weight of any other variable. Gender, type of degree, and department affiliation were not statistically significant. It was concluded that the more students were seen by the faculty as being academically prepared, appreciative, and interested, the more satisfied they were in their jobs. Time spent on class preparation and advising of students had a negative influence on job satisfaction.

Grandjean, Aiken, and Bonjean (1976) conducted an exploratory study to determine the importance and the satisfaction associated with 21 job characteristics in relation to professional autonomy in nurse educators. The researchers posed two questions: What is the relative importance to nursing educators of professional autonomy as 
compared with other characteristics at their work?, and What is the degree of satisfaction or dissatisfaction attributed to the potential discrepancy between autonomy and authority? A questionnaire, developed and validated by the authors, asked the respondents to rate 21 job characteristics (some examples include physical surroundings, opportunities to be a good teacher, salary, and fringe benefits). Subjects were told that other faculty had rated the characteristics as important aspects of an ideal job. They were instructed to indicate how important they would consider each characteristic as if they were interested in a position within the field. The subjects were then instructed to rate the job characteristics in relation to their current position. Two open-ended questions asked each respondent to discuss the "best thing" and the "thing they liked least" about their current position.

Grandjean et al. (1976) mailed a questionnaire to 191 nursing faculty members from four state universities in 1973-1974. The returned questionnaires (171) represented an $84 \%$ return rate. Those respondents who returned the questionnaire and whose rank was instructor or higher were chosen from the group for the study $(n=154)$. Analysis of the responses was by descriptive and correlation statistical methods. The responses indicated that for relative importance, the "opportunity to become a good teacher" $(M=3.5)$ was important to the subjects. The "opportunity to work with supportive colleagues" $(M=3.1)$ ranked second and tied with "keeping current in clinical knowledge." The fourth ranked characteristic was "a dean who lets me define my own responsibilities and fulfill them in my own way" $(M=2.8)$. Salary was ranked seventh. For relative satisfaction, the mean scores indicated low satisfaction with faculty participation in policy decisions ranked 16th $(M=1.8)$. The characteristic for quality teaching ranked seventh in satisfaction $(M=2.1)$. The autonomy provided be a nondirective dean was ranked 7 th. The major source of satisfaction is "a position in an attractive community," followed by "a position in a 
location permitting my spouse to follow his career." The least satisfying item was the “opportunity to do research" $(M=0.7)$. Importance and satisfaction scores were compared for each university, with all possible pairs of rankings compared and a rank-order correlation coefficient computed. There were considerable variations between schools. Grandjean et al. related the implications of the study to the importance of teaching faculty members and the autonomy provided within the college setting. This study was conducted 26 years ago, yet the opportunity to compare data gathered today would be very interesting, and perhaps similar in results.

Lubbert (1995) explored the relationship between dimensions of structure (centralization, formalization, and complexity) and climate in schools of nursing. Three research questions were asked: (a) the relationship between centralization and faculty perceptions of climate in schools of nursing, (b) the relationship between formalization and climate, and (c) the association between vertical complexity and climate. Contingency theory was used as a framework for the study. The target population was the entire faculty from all nursing programs, both baccalaureate and associate degrees in Minnesota, accredited by the National League of Nursing. All full-time faculty in the 21 nursing schools were invited to participate. Eleven of the 13 baccalaureate and seven of the eight associate degree nursing programs participated. A total of 187 questionnaires were mailed to the faculty. One hundred eighteen questionnaires were returned. One hundred and eleven met the requirements for the study (61.7\%). The study design was quantitative and the research method was correlational. The questionnaire consisted of four parts and was used to measure centralization, formalization (job codification, role observation, and job descriptions), and climate (involvement, peer cohesion, administrative support, autonomy, task orientation, work pressure, clarity, control, and innovation). Coefficient alphas for all 
parts of the questionnaire ranged from .57 to .95 . The independent variables were the structural variables and the dependent variable was climate.

Using Pearson product-moment correlational method, results revealed a significant and substantial relationship between mean total control (scores added from all five hierarchical levels) and climate. At the .001 level of significance, administrative support ( $r$ $=.59)$ was the most statistically significant; however, all factors were significant at the .05 level. Work pressure $(r=-.20)$ and control $(r=-.22)$ were negatively correlated. The results of the study indicated a statistically significant relationship between decentralization and favorable climate. Lubbert (1995) concluded that decentralization throughout a school of nursing is associated with a climate that is perceived positively by faculty. A favorable climate would be one with reduced work pressure. Schools with tight controls or a strong hierarchical structure would have a less favorable climate.

Kennerly (1989) examined the relationships between leadership behavior of the dean/chair, selected organizational characteristics, and nurse faculty job satisfaction. The researcher proposed four research questions: (a) What is the relationship between nurse faculty job satisfaction and perceived consideration behavior of the dean/chair? (b) What is the relationship between nurse faculty job satisfaction and initiating structure of the dean/chair? (c) What is the relationship between nurse faculty job satisfaction and the organizational characteristics of size, pay, structure, and tenure? and (d) What are the relationships among nurse faculty job satisfaction and perceived consideration and initiating structure of the dean/chair and organizational characteristics? Deans of 44 private colleges, listed in a publication from the National League of Nursing 1985-1986, were contacted for permission to approach their perspective faculty for the study. Twenty-six deans/chairs participated in the study with 246 full-time registered nurse faculty from 26 
programs. A mailed questionnaire with two parts was sent to all subjects. One hundred eighty-nine nurse faculty (77\%) participated in the final sample. The questionnaire included both the faculty member's rating of perceived leadership of the dean/chair and the faculty member's rating of his/her own job.

Kennerly (1989) developed a questionnaire to collect demographic data concerning the institution and program. There were five areas of interest: (a) nursing program size (number of full-time faculty and number of enrolled upper division majors), (b) institution size, (c) number of designated hierarchical positions with the program (structure), (d) range of salaries (pay), and (e) tenure, defined as a calculated numeric value which represented the potential advancement through tenure options. The Leadership Behavior Description Questionnaire-Form XII (LBDQ-XII) measured nurse faculty perceptions if the leadership behaviors of the dean/chair. Each of the ten subscales incorporated a 5-point Likert-type scale (performance ratings of never, seldom, occasionally, often, or always). Cronbach's alpha estimates of reliability were .78-.90. The Job Satisfaction Survey, an 18 item form with a 5-point Likert-type scale, measured the overall job satisfaction of the nursing faculty. Cronbach's alpha reliability was .85 .

Kennerly (1989) eliminated three programs because less than four respondents returned questionnaires. There were 181 faculty from twenty-three programs who participated in the study. The response rate was $79 \%$. Descriptive, correlational, and multiple regression statistical methods were used to analyze data. The mean number of program faculty in the study group was 11.8 and ranged from 6-22. Twenty-eight percent (51) held tenure positions. The number of faculty employed for longer than two years was $82.9 \%$. Salaries converted to a 10 -month figure ranged from $\$ 12,791$ to $\$ 31,667$; the mean salary was $\$ 19,171$. The correlational coefficient was computed for nurse faculty job 
satisfaction and the leadership behaviors of consideration and structure. Correlations for total scores of the Index of Job Satisfaction with subscores of the LBDQ-II were $r=.3732$ for consideration and $r=.3252$ for initiating structure. These were statistically significant and low-moderate in strength, suggesting that both consideration and initiating structure exert critical influences on the development of job satisfaction. The relationship between nurse faculty job satisfaction and the organizational characteristics of size, structure, pay, and tenure were not significantly different for any of the organizational characteristics.

Kennerly (1989) computed a multiple regression to examine obtained data for factors on consideration, initiating structure size, program structure, pay, tenure, and job satisfaction. Job satisfaction was the dependent variable and the characteristics of the organization were the independent variables. Regression weights for consideration $(r=$ $3.16, p=.00)$; initiating structure $(r=1.96, p=.05)$, number of nursing faculty $(r=$ $-2.38, p=.02)$, and number of college students $(r=-2.38, p=.02)$ indicated that these four variables contributed significantly to the prediction of nurse faculty job satisfaction. Job satisfaction in this study was high despite pay, structure, and tenure. Kennerly suggested that faculty might not perceive these variables as substantial job factors. Deans/chairs in these private colleges displayed a balance between the use of consideration and initiating structure.

Johnson (2001) explored the organizational culture and job satisfaction of Associate Degree nursing educators in order to ascertain their impact on faculty empowerment. The descriptive correlational study sampled 407 nursing educators in 70 associate degree nursing programs in the Southeastern United States. Instruments used to gather data were the Organizational Culture Assessment Instrument, the Job Satisfaction Scale, and the Psychological Empowerment Instrument. The researcher proposed three 
hypotheses: (a) the organizational culture of the $\mathrm{AD}$ nursing faculty is a contributing factor to the faculty empowerment, (b) $\mathrm{AD}$ nursing faculty job satisfaction is a contributing faculty to empowerment, and (c) there is a relationship between the organizational culture of AD nursing and their job satisfaction. The Competing Values Framework (CVF) was used as the theoretical framework for the study. There are three methods by which an organization can demonstrate its values: (a) the organizational structure of the value to which the organization ascribes, (b) flexibility and control or the internal and external factors that serve as the organizational objects of focus, and (c) the timing of the focus or the manner in which the work is done regardless of the outcome. Within the framework of the CVF, organizational dynamics are explored in relation to values that emerge as a result of the organizational culture or climate in the work environment.

Additionally, Johnson (2001) utilized the Demographic Informational Survey (DIS), a researcher-generated questionnaire for data collection consisting of personal and professional demographics, institutional demographics, and internal factors such as, age gender ethnic affiliation, basic nursing preparation, highest attained educational level, faculty rank, and number of years as nursing faculty in an $\mathrm{AD}$ program. Institutional demographics included setting and size of the nursing education program, including number of faculty employed and nursing enrollment. Other information concerning changes within the area were included: (a) recent curriculum revision, (b) recent changes in faculty responsibility, (c) new nursing education administrator, (d) change in nursing philosophy, and (d) recent changes in faculty responsibility. The Organizational Culture Assessment Instrument (OCAI) was a six-item ipsative response tool to assess organizational culture and values within the organization. Designed to measure the six dimensions of organizational culture--(a) dominant characteristics of the organization, (b) 
leadership style and approach, (c) management of employees, (d) organizational glue, (e) strategic emphases, and (f) criteria of success--the scale modified to a 24-item, 6-point Likert scale with responses ranging from 1 (strongly disagree) to 6 (strongly agree). Reliability measures for the OCAI were from .74-.80.

The Job Satisfaction Survey (JSS) was a 36-item nine-facet subscale measure to assess employee satisfaction about the job and various aspects of the job. These included: (a) pay, (b) promotion opportunities, (c) supervision, (c) fringe benefits, (d) contingent rewards, (e) operating procedures, (f) coworkers, (g) nature or type of work, and (h) communication. Reliability coefficients were validated from .60 to .91 (Johnson, 2001). The Psychological Empowerment Instrument (PEI), a 12-item, 7-point Likert scale measure with responses from 1 (strongly disagree) to 7 (strongly agree), was used to measure empowerment of $\mathrm{AD}$ nursing faculty. Cronbach's alpha scores were reported for the defining variables: meaningfulness $=.81$, competence $=.76$, self-determination $=.85$, and impact $=.83$.

The methodology of the study included multiple regression and correlational analysis. The independent variables were organizational culture and job satisfaction, and the dependent variable was faculty empowerment. A power analysis was performed prior to onset of the study to determine appropriate samples and effect size. At a confidence level of $95 \%$, a minimum sample of 311 subjects was required to decrease the risk for sampling error (Johnson, 2001). Surveys were mailed to 754 subjects and 408 responses were received (a $54 \%$ return rate). Results indicated $9.8 \%$ of the variability of faculty empowerment was accounted for by organizational culture. Therefore, the first hypothesis was accepted at the .01 level.

Johnson (2001) reported that job satisfaction was a statistically significant 
contributor to faculty empowerment $\left(F=131.87, p<.01\right.$ ), with $R^{2}$ of $24.5 \%$, while job satisfaction had a greater direct effect on faculty empowerment than organizational climate. However, there was found to be a moderate correlation between organizational culture and faculty job satisfaction. Thus, organizational culture scores were doubly linked to faculty empowerment, both directly and indirectly through job satisfaction for this sample of $\mathrm{AD}$ faculty. There were two demographic variables statistically significant for faculty empowerment: number of years as an $\mathrm{AD}$ nurse faculty member $(F=9.17, p<$ $.01), R^{2}=.022$, and faculty $\operatorname{rank}(F=6.40, p<.01), R^{2}=0.16$.

Several of internal program factors influenced faculty job satisfaction. Regression analysis of the data demonstrated that a new $\mathrm{AD}$ administrator was a statistically significant contributor to job satisfaction, although negative in direction, i.e., having a new AD nursing administrator decreased job satisfaction. Summarizing, turnover in faculty was found to be a statistically significant negative contributor to job satisfaction $(F=1.28, p<$ $.01), R^{2}=.032$. Overall, Johnson (2001) found that the organizational culture of AD nursing faculty was a statically significant contributor to faculty empowerment as was job satisfaction. Finally, an indirect statistically significant relationship was found to exist, from organizational culture through job satisfaction to faculty empowerment for $A D$ nursing faculty.

Moody (1996) conducted a study to ascertain the relationship between job satisfaction among nurse faculty and certain demographic variables, organizational characteristics, and role orientation. Demographic variables included: (a) nurse faculty age, (b) gender, (c) total number of years teaching experience, (d) degree programs currently taught, (e) highest degree attained, (f) tenure status, and (g) salary range. Organizational characteristics included: (a) type of institution (public or private), (b) total student 
population, (c) number of full-time nursing faculty, (d) presence of a tenure system, (e) presence of a collective bargaining unit, and (f) institutional support. Independent variables were demographic characteristics, organizational characteristics, and role orientation. The dependent variable was job satisfaction. The methodology included descriptive, correlational, and multiple regression analyses. The sample consisted of nursing faculty in 45 schools of nursing listed in the National League for Nursing (1988) publication. Three instruments developed by the researcher were used to collect data concerning demographic information, organizational information, and role orientation. Two standardized tests--the Job Descriptive Index (JDI) and the Job in General Scale (JIG)--measured job satisfaction. The JDI measured five areas of job satisfaction: (a) satisfaction with work itself, (b) pay, (c) opportunities for promotion, (c) supervision, and (d) coworkers. The reliability coefficients ranged from .80 to .90 . The JIG measured various aspects of the job to produce a score reflective of the respondent's feelings of overall job satisfaction. The overall reliability coefficients were .91 and .95 .

Moody (1996) sent a letter with a description of the study to deans of schools of nursing from the target population. After approval, 15 packets with a letter, research instruments, and return envelope were sent to each of the 45 schools. Eighty percent of the schools in the target population participated in the study. The overall response rate for faculty was $60 \%$ ( 308 of 511 ). The usable responses represented $56 \%$ ( 285 of 511 ) for the sample. In rank order, the nursing faculty members were most satisfied with work itself, supervision, the job in general, and coworkers. The opportunity for promotion and pay showed neither a negative or positive significance for the faculty. Median job satisfaction scores of the nurse subjects were compared to national norm scores previously established. Nurses in the study scored higher and were more satisfied with their opportunities for 
promotion and less satisfied as compared to the national comparison mean of the national group.

In the psychosocial subsystem of the organization, nurse faculty demographic variables that demonstrated statistical significance were number of years at the current institution, type of degree program in which faculty members teach, and salary range. There was a negative correlation between number of years at the current institution and nurse faculty satisfaction with opportunities for promotion. All demographic and organizational variables were initially included in the equation for step-wise linear regression analysis. The strongest relationship was between salary and job satisfaction, with a beta value of .296 . The higher the salary, the greater the job satisfaction was. The degree level of the nursing students was the second highest correlation. Faculty teaching students in master's and doctoral programs reported a higher degree of job satisfaction than faculty teaching students in baccalaureate or associate degree programs. Length of faculty contract was the third strongest variable. Faculty members on 9-month contracts were more satisfied with their job than those on 12 -month contracts $(B=-.1155)$. Moody (1996) discussed the statistical significance to pay and job satisfaction in the study and the concern with current economic resources. She suggested other methods to provide special benefits to faculty to maintain job satisfaction. The inverse relationship between years at current institution and satisfaction with opportunities for promotion present a problem for nursing education programs. Moody suggested that the promotional system be expanded to recognize accomplishments of the more experienced faculty.

Bonjean, Brown, Grandjean, and Macken (1982) conducted a longitudinal study to document whether changes in job satisfaction took place over time and whether the changes observed were to be expected on the basis of an original study and the follow-up 
after intervention plans. The researchers reported a gap in longitudinal studies demonstrating the effects of organizational change. The original research was conducted nine years earlier in a survey of nursing programs at four major universities in the Midwest, the South, and the Southwest. Findings from the research supported the generalization that nursing educators had a professional orientation and placed a high value on intrinsic rewards. Nurse educators ranked faculty input in determining school policy extremely low. The college that had the lowest overall satisfaction developed a plan to study the issue of faculty participation in decision-making. The Dean of that school assumed ultimate responsibility for the program, for the local organization, and administration, with faculty serving in advisory and consultative roles.

Committees had been assigned to develop a new set of procedures, implemented in 1974 and consistent with the debureaucratization implications of personality and organization theory. Six standing committees were established to conduct the business of the faculty, with an advisory committee to make recommendations directly to the dean regarding budget, faculty appointments, promotions, merit increases, and travel. In 1979, a second questionnaire was completed by 50 of the 59 full-time faculty members. Overall work satisfaction was measured for each faculty member. Each faculty completed the questionnaire rating their satisfaction with 21 different job characteristics. Results demonstrated that there was consistent evidence of an increase in work satisfaction between the two surveys. The average assessment score for all nursing faculty members at the nursing college in Texas in 1973 was .57 ; by 1979 , the mean was .74 . Faculty were also asked to complete a direct question with responses using a 5-point Likert-type response scale whether the school had become a better or worse place to work. Seventy-six percent rated their working conditions as "much better" or "somewhat better." When asked 
how they felt about the new organizational structure as compared to the previous one, $70 \%$ said they were "much more" or "somewhat more" satisfied under the new structure. Twenty-five percent expressed less satisfaction. Bonjean et al. (1982) cautioned that this was a "natural experiment"; thus rigorous experimental controls could not be incorporated and causal influence of organizational change on faculty morale cannot be conclusively inferred. In both surveys the intrinsic factors were evaluated. Major changes in the responses on the questionnaire include an evaluation of physical surroundings which went from a ranking of 20th in 1973 to third in 1979. The characteristic that stated, "Dean who lets me define my own responsibilities" was ranked 13th on the 1973 scale and sixth in 1979. The researchers warned that the traditional hierarchical system with a "boss making all of the decisions is no longer workable in nursing....Decentralizing authority in a professional organization modifies the relationship between leaders and followers and can bring about substantial increases in the work satisfaction of both" (Bonjean et al. 1982, p. $368)$.

In a study done by Garbee and Killacky (2008), nursing faculty were surveyed as to their intent to stay in their position. The researchers were interested in what variables were likely to predict a satisfied faculty member who was intent on staying from one, three, and five years. Selected variables of job satisfaction, mentoring, organizational commitment, and leadership behaviors were measured. The research questions included: (a) what is the relationship between job satisfaction and intent to stay in nursing education, (b) can mentoring experiences predict a nursing faculty's intent to stay in education, (c) is organizational commitment to the school of nursing predictive of faculty intent to stay in nursing education, and (d) are faculty perceptions of their dean's leadership behaviors predictive of faculty intent to stay in nursing? A random selection of full-time nursing 
faculty teaching in the Southern Region Education Board (SREB) states were divided into three groups based upon the level of nursing shortage from low to high. One research instrument containing four parts--an Index of Job Satisfaction, the Dreher and Ash Mentoring Scale, the Organizational Commitment Questionnaire (OCQ), and the Leader Behavior Description Questionnaire--was administered using an on-line format. Additionally there were six questions dealing with the nurse faculty member's intent to stay. At the end of the instrument were three open-ended questions or statements that asked the respondent to identify factors that contributed to their satisfaction and dissatisfaction from their work.

Garbee and Killacky (2008) received support in writing from all nursing deans in the SREB region colleges having nursing programs. Personnel responsible for forwarding the survey link to faculty were sent an e-mail message with instructions. There were 316 usable responses from the 782 potential faculty members ( $40.4 \%$ response rate). The study results demonstrated that the group was more satisfied than dissatisfied. Satisfied responses were from "Being Part of Student Success" (20.1\%) down to "Image of Excellence" (1.8\%). The comments of dissatisfaction were "Time Demands" $(29.3 \%)$ to "Lack of Faculty" (1.7\%). A cross case analysis was divided into the four themes of individual, institutional, leadership, and mentorship. Identified under individual were positive responses as being part of student success, yet the response relating to student attitudes was a negative. Under institutional, collegial environment was a positive yet time demands, low pay, and long commute were considered negatives. Bureaucracy, extremes in leadership and time demands were listed as negative themes under leadership, while supportive chair, administrator, dean were identified as positives. Participants did not rate mentoring as a predictor to stay with the organization but the longer the contract length (12 
months) and the faculty with the most education had a significantly higher response rate. Although there was not a significant difference in intent to stay scores between groups, there was a significant difference in the mean scores for faculty working 40 hours per week $(M=8.24, S D=2.48)$ who intended to stay three years than those working 60 hours $(M=$ $7.13, S D=2.45$ ). The results from the Garbee and Killacky study supported a similar study by the NLN in 2005 .

The preceding section has dealt with job satisfaction in relation to organizational structure; the next group of research studies examines role conflict and role strain in nursing faculty. Two of the studies focus on clinical faculty.

\section{Role Conflict}

Fain (1987) compared nurse educators' perceptions of role conflict and role ambiguity with reported facets of job satisfaction, role conflict and role ambiguity. Role conflict was identified when there are conflicting role expectations and demands. Role ambiguity was not having accurate and clearly articulated expectations of behavior or expected performance. The research method included a series of one-way multivariate analysis (MANOVA) with a post hoc multiple comparison test (Scheffe). A hierarchical multiple regression was used for analysis. The independent variables were derived from the demographic survey and the dependent variables were the five facets of job satisfaction, role ambiguity, and role conflict. The investigator asked two research questions: Is there a difference in job satisfaction, role conflict, and ambiguity among faculty? and Is there a relationship between perceived levels of role conflict and role ambiguity with the five facets of job satisfaction: (a) work, (b) co-workers, (c) pay, (d) supervision, and (e) opportunities with promotion?

Fain's (1987) sample consisted of baccalaureate programs in six New England 
states (27 programs). The convenience sample included individuals employed full time in baccalaureate programs in nursing who volunteered to participate. Deans of each program were contacted by mail and asked to submit to the researcher all names of full-time faculty teaching in baccalaureate programs. Packets containing the instruments were mailed to participants. Of the 387 eligible subjects, 285 returned the completed questionnaires (74\%). The Role Questionnaire (RQ) was a 14-item scale to assess perceived areas of role conflict and role ambiguity with a 7-point Likert-type scale. A score of 1 indicated the statement was not true of one's job. The higher the score obtained, the higher the levels of role conflict and role ambiguity. The internal consistency for RQ was .81 and .86 for the two scales, respectively. The Job Descriptive Index (JDI) was used to measure the five facets of job satisfaction: work, co-workers, pay, supervision, and opportunities for promotion. Respondents indicated how well each short phrase described their job. Internal consistency ranged from .80-.89. A demographic survey was also included.

The five facets of job satisfaction differentiated among faculty according to age, years of university teaching, level of education, and present position. Satisfaction of pay was the only job facet to differentiate significantly between all four background variables. The post hoc Scheffe test for satisfaction with pay indicated that faculty over 46 years of age were more satisfied with their pay than any other group $(M=19.93)$. In a hierarchical multiple regression, role ambiguity accounted for $28.5 \%$ to $4.3 \%$ variance for the five facets of job satisfaction. Fain's (1987) results supported other research on role conflict and role ambiguity. However, the significance of job satisfaction in relation to pay when the faculty member was over 46 years of age and with more than 16 years of teaching experience supports the research of Moody (1996), but does not support the findings of the earlier research of Granjean et al. (1982). 
Piscopo (1994) examined the differences between clinical nursing faculty (CNF) perceptions of organizational climate and communication within affiliating agencies. The research hypotheses were (a) Clinical nurse faculty and nursing unit managers differ in terms of their perceptions of organizational climate and communications within the affiliating agency, (b) For clinical nurse faculty, perceptions of both organizational climate and communication were positively correlated with role strain, and (c) For clinical nurse faculty, role strain is linearly related to the predictor variables of organizational climate, communication, length of time on the nursing unit, and length of time working with the nursing unit manager. The target population was full-time clinical nurse faculty who oversaw student experiences in acute care in-patient settings and who were employed in baccalaureate nursing programs within the New York metropolitan area. The researcher met with each institution as a group. Ninety-eight nursing faculty from the nursing programs and the nursing unit manager with whom they worked participated in the purposive sample.

Faculty were given a nursing unit manager identification form, faculty data form, and an Organizational Climate Within Questionnaire, A Communication Within Organization Organizations Questionnaire, and a Role Strain Questionnaire. The Organizational Climate Questionnaire consisted of 47 items with a 4-point Likert-type scale response format with a reliability of .74 . This was used to measure perceptions held by the clinical nurse faculty and nursing unit managers of the organizational climate or atmosphere within the affiliating agencies. A higher composite score was reflected in a positive response to organizational climate (Piscopo, 1994). The Communication Within Organizations Questionnaire consisted of 35 items using a 7-point Likert-type scale response format with a reported reliability of .70 . This was used to measure the perceptions 
of communication or flow of information within affiliating agencies. A positive response to communication was reflected in a higher composite score. The Role Strain Questionnaire, used to measure the degree of difficulty faculty experience while they were overseeing the clinical practice of nursing students, was a 32-item instrument that used a 5point Likert-type scale response format with a reported reliability of .90. A high score reflected less strain, a lower score reflected greater strain. Faculty were asked to identify the nursing unit manager with whom they worked during the semester and to complete all of the questionnaires.

Piscopo (1994) utilized a correlational design with multiple regression to analyze the data. Questionnaires from 31 faculty and their respective unit managers were completed with a $30 \%$ return rate. Faculty reported that they had been with the affiliating agency for an average of 48.48 months $(S D=50.25)$. They oversaw the clinical practice of nursing students on the identified unit for an average of 36.19 months $(S D=28.68)$, and they had been working with the identified nursing unit manager for an average of 12.94 hours per week $(S D=11.97)$. Ninety per cent of the clinical nurse faculty reported that preparation for teaching was included in their master's degree program, and $70 \%$ reported that clinical practice was included in their master's degree program. Seventy-five percent of the 28 faculty indicated that preparation for teaching included a clinical practicum focusing on the clinical supervision of nursing students.

Differences in perceptions of organizational climate and communication between the CNF and the nursing unit managers were analyzed using an independent $t$ test for the total scores. Perceptions held by the clinical nurse faculty and nursing unit managers of organizational climate were not statistically significant. CNF and nursing unit managers held very different perceptions of communication within the organization $t(60)=-3.95$, 
$p<.01$. The average score for unit managers was $170.48(S D=17.58)$ whole the average score for the CNF was $154.23(S D=14.69)$. Perceptions of the CNF were neutral, but nursing unit managers held a more positive perception of communication. Clinical nurse faculty were less likely to view communication within the organization positively due to the possibility of being "outside" of the organization; they tended to enter the organization only with students and had to rely on nursing personnel to update and communicate fully with nursing faculty (Piscopo, 1994). Pearson product-moment correlation of coefficients was computed between the research variables: (a) organizational climate, (b) communication, (c) time in agency, (d) time on the unit, (e) time with the nursing unit manager, and (f) the presence of role strain. The CNF did not report a high degree of role strain. Communication was positively correlated to organizational climate $(r=.43, p<$ $.01)$, and role strain $(r=.54, p<.001)$. Organizational climate was positively related to role strain $(r=.39, p<.01)$ and role strain was positively related to both time on the unit $(r$ $=.54, p<.001)$ and time with the nursing unit manager $(r=.41, p<.01)$. Time on the unit positively related to time with the nursing unit manager.

Piscopo (1994) conducted a stepwise multiple regression analysis to determine which variable best predicted the degree of role strain reported by the CNF. Communication explained $29 \%$ of the variance, while time on the nursing unit and communication explained $36 \%$ of the variance in role strain. The predictive value of other variables--organizational climate, time with the nursing unit manager, and time in the agency--were negative when entered into the equation. Significant differences in perceptions of organizational climate between CNF and nursing unit managers were not found. Similar experiences when involved in practice may have influenced the faculty's perception to be more similar to that of the nursing unit manager. There was a significant 
positive correlation between perceptions of organizational climate and role strain. Where perceptions of organizational climate by CNF were positive, CNF reported less strain. Likewise, where perception of communication was positive, faculty reported less strain.

The nursing literature is lacking in research studies on the orientation of CNF to an affiliating agency in which the faculty person is assigned to oversee the clinical experiences of nursing students. Frequent moves of CNF from one unit to another or one course to another means that the faculty have to re-establish meaningful communication patterns with other different agency members, a task that eventually takes its toll on the faculty in terms of role strain (Piscopo, 1994).

Oermann (1998) also studied role strain in clinical nursing faculty, examining differences based on the education of the faculty member, level of nursing students for whom they are responsible, and employment status (full-time or part-time). Role strain is the subjective emotional response of the individual to the situation. If the person responds with tension and frustration, it may intensify and may indicate role strain. Role strain often intensifies as the number and complexity of the demands within a role increase, causing difficulty in meeting multiple roles. Clinical nursing situations can create problems and place the faculty member at risk for developing role strain. The demands of supervising students in the clinical setting and safeguarding their patients and well being can be emotionally as well as physically exhausting. Contributing to role strain may be the extensive knowledge and skills to be developed in their students, the acuity of the patient, and the number of patients for whom students care. Clinical agency personnel may expect certain role behaviors from the instructor, which may differ considerably from those expected by the nursing program.

Using a descriptive and correlational exploratory design, Oermann's (1998) sample 
consisted of full-time and part-time clinical facility $(N=226)$ from 20 randomly selected associate degree and bachelor's degree programs in the Midwest, accredited by the National League for Nursing. The research questions asked: (a) What is the degree of role strain among clinical nursing faculty, and (b) Are there differences in the role strain based on educational preparation of the clinical teacher, level of nursing students for whom the teacher was responsible, and employment status (full- and part-time)? The instrument, the Role Strain Scale, Part I, had 44 items with an alpha coefficient of 0.96. Part II assessed socialization experiences and demographic characteristics. The adapted instrument consisted of 23 statements describing potential sources of stress for clinical faculty. A behavioral frequency scale from 1 (never) to 5 (nearly all the time) was used to assess the degree to which each statement describing work-related situations that were, or had been, a source of stress for the respondent. The instrument provided scores for seven basic subscales: (a) Intersender Conflict, demands of one person in a role set conflict or are incompatible with the demands of another person in the role set, (b) Intrasender Conflict, demands of the role are incompatible or mutually exclusive, (c) Interrole Conflict, demands of one role if the faculty conflict with demands of another role, (d) Role Ambiguity, degree to which clear information is lacking on the expectations associated with the role, (e) Role Overload, role expectations that are too complex, that make too many demands for the time and energy available or that create conflict between quality and quantity given time constraints, (f) Role Ambiguity, one's personal beliefs and values are not consistent with the role expectations, and (g) Role Incompetence, the individual in the position is not able to fulfill the role due to lack of knowledge or skill. The alpha coefficient for the total scale was .93; the subscales measured as follows: Intersender Conflict $(\alpha=.51)$, Intrasender Conflict $(\alpha=.71)$, Role Overload $(\alpha=.86)$, Role 
Incongruity $(\alpha=.79)$, and Role Incompetence $(\alpha=.80)$. Oermann did not calculate the alpha coefficient for the Role Ambiguity and InterRole Conflict subscales.

Both associate degree $(n=100)$ and bachelor's degree $(n=126)$ clinical faculty participated in Oermann's (1998) study. The mean age was 46 years of age; more than half of the faculty (64.6\%) was prepared at the master's level. There were 35 clinical teachers in the BSN program with Doctorate degrees compared with 9 in the ADN program. Faculty had a mean of 12 years $(S D=8.6)$ experience as clinical teachers (range 1-35 years). Role strain was analyzed by calculating a mean score for the seven subscores and a total score for role strain experienced by faculty. The highest mean scores were for the subscales of Interrole Conflict $(M=3.36)$, Role Overload $(M=3.32)$, Intersender Conflict $(M=3.32)$, and Intrasender Conflict $(M=3.0)$. The total Role Strain score was $2.90(S D=$ 8.6), which reflected a low degree of role strain. The Student-Newman-Keuls test was used to identify differences based upon the educational level of the faculty. The clinical faculty with doctoral degrees experienced the most role strain, Role Overload $F(5,215)=5.96, p<$ .001 ; Interrole Conflict $F(5,215)=3.26, p<.007$; and Intersender Conflict $F(5,215)=$ $3.86, p<.002$.

Clinical faculty teaching sophomores in the BSN program had the highest Total Role Strain scores compared with teaching other levels of students as indicated by the StudentNewman-Keuls test. Full-time faculty experienced significantly higher Total Role Strain scores and higher subscales scores except for Role Incompetence. The highest score for Role Overload was for full-time faculty $(M=3.44, \mathrm{SD}=.78)$, part-time faculty scored lower $(M=2.92, \mathrm{SD}=.57)$. Faculty teaching clinical courses in ADN and BSN programs experienced a low degree of role strain in contrast to those who had research responsibilities, who reported higher role strain than clinical faculty. In comparison to 
ADN faculty, BSN faculty experienced more role conflict. The amount of time one spends teaching may leave no time for research and publication in four-year colleges. Clinical faculty who taught sophomores in baccalaureate programs experienced the most role strain. This may relate to responsibilities for support and guidance for new students in the clinical setting and the more complex skills required of students above the fundamental skills level. Clinical teachers must monitor their own time and learn to balance the multiple demands of their role. An adequate orientation to the clinical setting, consistency in clinical teaching assignments, and support from colleagues may assist faculty in coping better with the many responsibilities with clinical teaching (Oermann, 1998).

Steele (1991) noted that role strain can occur when there is difficulty in fulfilling role demands. Following recent studies, which had identified sources of stress to nursing faculty associated with the required multiple role functioning, the author examined the relationship between clinical practice, faculty perceptions, and perceptions of role and role strain. The study looked at the perceived importance of faculty who practiced clinically or who did not. The study also examined differences of perception relative to age, rank, and teaching and clinical experience. The researcher proposed two hypotheses: (a) there will be no statistical significant difference between practicing and nonpracticing faculty's perceptions and attitudes regarding importance of clinical practice as a role function, in maintaining clinical competence and in facilitating research and teaching effectiveness and (b) there will be no statistically significant difference between practicing and nonpracticing faculty's perceptions of role strain by level of education, years of teaching, previous clinical experience, faculty, rank, and clinical practice activity as a faculty member.

Steele (1991) defined faculty practice as care activities performed directly or personally by the faculty member that have the client as sole focus for which the faculty 
member is accountable and not including care provided indirectly through student supervision. The main forms of practice consists of moonlighting or work that is done on faculty's own time, and they are reimbursed directly for their services and students are not involved.

Letters for rosters of full-time faculty were sent to 100 deans or administrators of $\mathrm{NLN}$-approved nursing programs chosen randomly from four NLN geographic areas. The Dean Demographic Questionnaire (DDQ) was developed by Steele (1991) to determine faculty's perceptions of the importance of various faculty roles. Fifty-three programs were in the final sample and 550 questionnaires were mailed to individual faculty. Three hundred two were returned (55\%) and 292 were timely, usable, and included all of the data. Descriptive and correlational methods were used to analyze the data. The demographic results indicated that $97.6 \%$ of the respondents were women. They were assistant professors, $56.1 \%$; associate professors, $25.3 \%$; professors, $2.8 \%$, and instructors, $15.2 \%$. Forty-three percent of the subjects were tenured and 107 were on a tenure track. Of the 302 that responded, 122 faculty stated that they engaged in clinical practice, while 180 stated they did not.

Steele (1991) noted that the two groups, practice versus non-practice, were compared for differences. The dependent variable, perception of practice, was measured by the Faculty Perception of Practice Questionnaire (FPPQ). Role strain was determined by an adaptation of an instrument to determine role conflict ambiguity and overload among clinical nursing faculty. (There was no Cronbach's alpha included.) The cutoff point for accepting the null hypothesis was set at the .05 level. Practice was the independent variable with role perception and role strain as the dependent variables. The Deans rated four functions in the order of significance, as they perceived them: teaching, research, practice, 
and service. Non-practicing faculty perceived the importance of the role functions as teaching, research, service and practice. Practicing faculty ranked them teaching, practice, research, and service. Faculty evaluated their perception of clinical competence in their own teaching area. Non-practicing faculty rated their clinical competence as expert, $24.7 \%$; slipping occasionally, $57.6 \%$. For practicing faculty $52 \%$ rated themselves as expert and $5 \%$ recorded a need for an update. To keep up with skills non-practicing faculty relied primarily on care they gave to patients during the course of clinical teaching (75.3\%). Fourteen percent stated they had not maintained their skills. Of the practicing group, $7.5 \%$ had a joint teaching and clinical appointment and $92.5 \%$ relied upon supplemental activities of nursing care of patients apart from their faculty position. Faculty ranked 24 statements on selected aspects of practice related activities. Faculty that practiced, reported being perceived as a student role model more often than the nonpracticing group $(p<.05)$. Significantly more practicing faculty felt that increased faculty practice would improve the quality of nursing care $(p<.001)$. Nonpracticing faculty felt that practice expectations should reduce the educational demands of college and community service responsibilities.

Steele (1991) reported that when asked specifically about perceived role strain, the two groups did not differ significantly. When categorized on a continuum of 1 to 5 (1 being excessive and 5 being little to none) mean scores for both groups were two, indicating a large amount of stress. The 27-item, 5-point Likert-type scale concerning role strain was tested to the demographic variables of current title, highest degree earned, number of years spent in nursing, and years of employment. Fourteen items were found to be statistically significant at or beyond the .05 levels for the variables of rank, education, and experience. (Exact values for each variable were not included with the article.) Amount of perceived strain was greatest among assistant professors, with $61 \%$ 
feeling excessive to large amounts of role strain. Associate professors were at $56 \%$ and instructors, $50 \%$. Masters prepared faculty responded that there was more responsibility to be a role model for staff and were more confident in knowing what the school expected of them in the clinical area. A larger number of experienced faculty were more certain when to intervene when students gave care but had more difficulty in balancing teaching and professional responsibilities. Role strain seemed to increase with time. Excessive amounts of role strain were reported by $40 \%$ of the faculty with more than one year of teaching experience, steadily increasing to more than $92.7 \%$ in those up to 7 years (Steele, 1991).

The previous articles were focused on retention of faculty through reviewing research on role strain, following are research studies dealing with burnout in the nurse educator and interventions to retain excellent faculty.

\section{Burnout in Nursing Education}

Edelstein (2000) conducted a descriptive study to analyze burnout levels of nurse educators in state supported baccalaureate degree nursing programs in Wisconsin. Citing the concern that nurse educators are constantly dealing with stressors of change in the professional role, Edelstein wanted to discover what impact age of the nurse educator has in relationship to job satisfaction. Adding to the pressure of the educational program and the nurse educator as they struggled to maintain quality and clinically competent graduates were reductions in financial resources in higher education as well as financial competition among health care agencies. Nurse educators differ from other educators in that they work in two stressful environments: the academic setting and the clinical setting. Four research questions posed were: (a) Do nurse educators perceive themselves as experiencing burnout? (b) Do nurse educators feel satisfied with role functions of a faculty member? (c) Does middle age make a difference in these perceptions? and (d) Does a relationship exist 
between burnout and job satisfaction? The target population was 14 baccalaureate and higher degree programs in the state of Wisconsin, including four public schools through the University of Wisconsin System. There were 122 full-time faculty employed in the programs.

Three instruments were used for data collection. The Maslach Burnout Inventory (B), the MBI educator's version, was chosen for its self-administered 6-point Likert scale addressing emotional exhaustion, depersonalization, and personal accomplishment. Reliability estimates for Cronbach's alpha ranged from .88 to .90 for emotional exhaustion, from .74 to .76 for depersonalization, and from .72 to .76 . for personal accomplishment (Maslach, Jackson, \& Leiter, cited in Edelstein, 2000). The Faculty Satisfaction Questionnaire (FSQ ) was used to obtain information on the traditional academic professor roles. The FSQ was a 37-item, five point Likert scale designed to measure faculty satisfaction concerning teaching, research, and service. No psychometric information was given. The third instrument was a demographic survey, developed by the researcher, to obtain information on age and work schedule information.

Edelstein (2000) sent a packet with the questionnaires to the 122 faculty. Sixty-six questionnaires were returned to the researcher with a response rate of $54 \%$. A mean score was computed for the three components of the Maslach Burnout Inventory and the three components of the Faculty Satisfaction Survey. The data obtained from the demographic survey were analyzed for similarities and differences. To evaluate consistency, the means and standard deviations for the sample group were compared to the national means and standard deviations of teachers. To determine faculty satisfaction each of the three areas was analyzed for frequency, mean, and standard deviation. The means within groups of the three categories was compared using analysis of variance (ANOVA). Sixty nine percent 
(69.7\%) of the respondents were between 39 and 56 years of age, indicating that they are in the target population, corresponding to "baby boomer" classification. Seventy-four percent (74\%) of the respondents held the rank of clinical instructor, assistant professor, or associate professor; $48.5 \%$ had taught over 12 years.

Scores on the MBI were broken down into the three areas. For emotional Exhaustion (EE), 44\% of nurse educators perceived themselves at a high level, 27\% perceived low level, and $29 \%$, moderate. With $73 \%$ having moderate to high exhaustion, there was cause for concern. Almost $60 \%$ expressed feeling burned out, ranging from every day to once a month. In the area of Depersonalization (DP), nurse educators chose the value "never" for several statements (signifying the caring nature of nursing). The statement, "I feel some students blame me for some of their problems" had the highest mean score $(M=2.02, S D 1.45)$. The overall score for depersonalization was high $(M=$ 15.33, $S D=2.51)$. In the area of Personal Accomplishment (PA), high scores correlated with low burnout levels, and $65 \%$ of the nurse educators were in this range. Edelstein (2000) noted the most impressive response by the nurse educators (92.4\%) was "I have accomplished many worthwhile things in this job," ranging from everyday to once a month. Only two nurse educators reported the combination of high emotional exhaustion, moderate depersonalization, and low personal accomplishment. Thirteen of the nurse educators had low emotional exhaustion, low depersonalization, and high personal accomplishment, indicating a low degree of burnout. Comparing means and standard deviations in each category to national means and standard deviations, the nurse educators were most similar to the teaching subgroup scores. The nurse educator mean scores for emotional exhaustion were slightly higher overall than the teaching group.

On the Faculty Satisfaction Questionnaire (FSQ), there were 27 items, with 
responses on a 5-point Likert scale from (5) very satisfied to (1) very dissatisfied. The statement rated the highest by the respondents was 4.29 for academic freedom and the importance of designing instructional materials for teaching. In the area of research activities, the mean was 2.77 for supporting career development. Only $6 \%$ responded as satisfied to very satisfied with the overall research category. In the area of service, $30 \%$ of the respondents ranked the category as neutral, while $32 \%$ were satisfied. Placing the data into a one-way analysis of variance for the roles of research, teaching, and service produced a significant finding $(F=2.384, p=.007)$. Post hoc tests revealed no significant difference in the group's teaching and service, but there was a distinction in service and learning. Edelstein (2000) computed a one way ANOVA for the three categories of burnout for nurse educators in the 39-56 age range and for all nurse educators. Emotional and personal exhaustion by personal accomplishment was significant at the $p<.05$ level for the baby boomer group $(F=3.845, p<.001)$.

When scores of the nurse educators for the MBI and the FSQ were correlated, there was no significant relationship between research and emotional exhaustion. There was a negative relationship between service role and depersonalization, service role and emotional exhaustion, and service role and personal accomplishment. The correlations were significant at the 0.05 level (two-tailed): roles of teaching and depersonalization ( $r=$ $.295)$ and teaching and emotional exhaustion $(r=.260)$. In summary, Edelstein (2000) noted that $27 \%$ of nurse educators felt emotionally exhausted but were not burned out or overworked. In the area of depersonalization or negative attitudes and feelings concerning students, $66 \%$ reported low levels of burnout. Any level of depersonalization between the students and educator becomes a barrier to learning. Although teaching is a role that is satisfying for nurse educators, institutional rewards for teaching were not perceived as such 
and workload was a sensitive issue. With economic constraints, workload has generally increased, adding to the perception of dissatisfaction (Edelstein, p. 92).

Clinical supervision was not evaluated in this study but seems to be the role expressed as providing the most satisfaction. Clinical opportunities provided for more internal rewards with nurse/client interactions and excitement for student learning. This topic needs to be further explored in relation to workload issues. When preceptors or adjunct clinical instructors are hired to do clinical for full-time faculty, the area that is enjoyed the most is given away (Edelstein, 2000, p. 93). Analysis of the perceptions of burnout and job satisfaction yielded no statistical significance on mean scores comparing baby boomers and other educators. More research on the Burnout inventory is necessary in light of other work in the clinical area.

Staurovsky (1992) examined levels of burnout and job satisfaction to determine the relationship between burnout and job satisfaction in nurse educators and identified job related activities perceived as excessive stress producers. The study used descriptive statistics, Pearson $r$ correlation, and stepwise multiple regression to arrive at the results to the seven research questions: (a) To what degree are nursing faculty experiencing burnout? (b) To what level of job satisfaction is nurse faculty experiencing? (c) Do levels of burnout correlate with job satisfaction/dissatisfaction? (d) Is there a significant relationship between certain demographic variables and scores on the Maslach Burnout Inventory (MBI) and the Job Descriptive Index (JDI)? (e) What demographic variables are common among nurse faculty having high scores on the MBI and low scores JDI? (f) Are the number of identified stressors predictive of higher MBI scores in Emotional Exhaustion and Depersonalization and lower JDI scores on the Job In General section? and (g) What factors are most frequently identified as creating a sense of excess stress to the individual? 
Staurovsky's (1992) sample consisted of nursing faculty at three schools of nursing in one southwestern state. This area was chosen because of the similar environments of societal and organizational perspectives, including the effects of state budget constraints. All full-time faculty were included and administrative or joint appointed faculty were excluded. The potential sample size was 115 participants. From this sample 94 (82\%) questionnaires were received. After elimination for various reasons, 82 were usable (71\%). A four-part questionnaire was utilized: a section containing demographic and job related background information; the MBI to assess burnout; the JDI, an instrument to determine job satisfaction; and the Self Perceived Excessive Job-Related Stressors, educator form, consisting of 22 items that focused on frequency of job related feelings from Never (0) to Every Day (6). The three subscales for the MBI were Emotional Exhaustion (EE), Depersonalization (DP), and Personal Accomplishment (PA) with reported reliability coefficients for each subscale of $0.82,0.60$, and 0.80 , respectively.

The JDI measured job satisfaction in five distinct areas: (a) Work on Present Job (WPJ), (b) Present Pay (PP), (c) Opportunities for Promotion (OFP), (d) Supervision on Present Job (SPJ), (e) People and your Present Job (PPJ), and (f) Job in General (JIG). The 72 items consisted of short phrases or one word items that required the respondent to designate "yes," "no," or "cannot decide." Staurovsky (1992) reported reliability for all the scales was 0.80-0.91. The Self Perceived Excessive Job Related Stressors consisted of three open-ended statements for faculty to identify job related activities perceived as excessive stress producers; these were rank ordered from the greatest stressor to the least.

Staurovsky (1992) reported that the majority of respondents were female, ranging in age from 28-65 years with a mean of 45.9. Eighty-five percent (70) were not tenured. The respondents ranked clinical, classroom, and research as the most frequently occurring 
activities. The MBI results placed the nurse educators in the moderate range of burnout ( $M$ $=16.99$ ), the moderate range being 14-23. The Depersonalization mean was 3.16 , or in the moderate range in the study sample with the range being 3-8. The Personal Accomplishment study mean was 38.39 with the moderate range for PA being $36-42$. When compared to national norms of educators, there was little difference.

The JDI results showed that study participants scored similar to the JDI national norms (male norming population). JDI factors rated by at least $90 \%$ of the respondents were the following terms: "satisfying," "good," "useful," "challenging," and "gives sense of accomplishment." The lowest rated terms were "simple" and "boring," $2.4 \%$ and $7.3 \%$, respectively. On the subscales the educators generally responded positively; however responses to "present pay" and "opportunities for promotion" were negative or neutral. Responses to the factor "bad" were equally divided between "yes" and "no." Ninety-five percent responded "no" to the variable, "highly paid." Results examining predicted high burnout were not substantiated on the Emotional Exhaustion Scale, and there was moderate burnout on the Depersonalization and Personal Accomplishment Scale. Staurovsky (1992) had hypothesized a high level of job satisfaction on the Job Descriptive Index and five of the six scales supported this; however, the Present Pay subscale demonstrated low satisfaction with the job. A chi-square analysis revealed a significant difference on four of the six scales, but "Present Pay" and "Opportunities for Promotion" were not significantly different with the majority in the low satisfaction range.

Staurovsky (1992) predicted that the faculty who demonstrated high burnout based on the Maslach Burnout Inventory would have low job satisfaction scores on the Job Descriptive Index. Pearson $r$ correlations were use to determine the significance of the relationships to the scores on the three MBI subscales and the six JDI subscales. Highlights 
of these and other results included the following: cumulative effects of the Work on Present Job (WPJ), Present Pay (PP), Opportunities for Promotion (OFP), and People and Present Job (PPJ) subscales accounted for $56 \%$ of the variance in the Emotional Exhaustion subscale. Eighty-one percent (81\%) of the sample population was experiencing high burnout on one or more of the MBI and/or JDI subscales. One subject was totally burned out and eight individuals were termed "terminal burnout," scoring at great risk for burnout with high scores on two scales. The highest number of burnout responses was on the Personal Accomplishment subscale. Another major concern in the study was that $60 \%$ of the study group reported low satisfaction with pay.

Teaching in a nursing program can cause high levels of stress and lead to burnout. Burnout is a response to stress and is commonly seen in individuals who are part of the helping professions. Nursing and teaching are two professions where burnout is evident, according to Talbot (2000) who conducted a study to assess the correlation of burnout among community college nursing faculty and their use of humor to reduce academic stress related to burnout. Previous studies had examined the use of humor to reduce the academic stress related to burnout at the baccalaureate and associate degree level. Based on previous research on burnout and coping with humor, Talbot posed four research questions: (a) To what degree does burnout occur in community college nursing faculty members? (b) To what extent do nursing education faculty members use humor as a coping mechanism,?(c) Is there a relationship between burnout and humor among community college nursing faculty? and (d) Is there a difference in burnout between community college nursing faculty members who have high humor usage to those with low humor usage?

Talbot's (2000) population was 90 community college nursing faculty members 
from a major metropolitan area in Texas. The names were obtained from four college catalogues and their faculty lists. Current teaching faculty listed in the catalog received a cover letter and a survey packet. Each faculty member completed an academic profile survey, the Coping Humor Scale (CHS), and the Maslach Burnout Inventory (MBI). Sixtythree questionnaires were returned with a response rate of $70 \%$. The academic profile survey contained questions concerning age, tenure status, academic rank, highest degree held, and workload. The CHS, a seven-item scale with four levels of responses had an internal consistency of $\alpha=.76$. The MBI, a 22-item survey reporting job related feelings on a seven-point scale, had an internal consistency of $\alpha=.80$ for Emotional Exhaustion, $\alpha$ $=.64$ for Depersonalization, and $\alpha=.77$ for Personal Commitment.

Talbot (2000) reported the results in three areas: demographic, burnout, and humor. A $t$ test was used to compare the results of community college nursing faculty first to postsecondary educators and then to medical practitioners. There was a statistically significant difference in Emotional Exhaustion (higher scores equal greater burnout) in comparison to the other two role groups. The direction was lower for nursing faculty ( $\mathrm{D}=$ -2.78 points, $p<.01)$ as compared with postsecondary educators and even lower against medical practitioners $(D=-6.40, p<.001)$. The MBI subscale for Depersonalization similarly demonstrated that medical practitioners and postsecondary educators scored much higher than nursing faculty $(D=-3.2$ and -1.65 , respectively). Talbot also found that community college nursing faculty scored significantly higher in Sense of Personal Accomplishment (higher scores equal less burnout) than medical practitioners and postsecondary educators with effect sizes of 1.39 and .85 , respectively.

Talbot (2000) compared the scores on the humor and burnout survey using a Pearson product-moment correlation with the total humor scores versus each of the three 
MBI dimensions (Emotional Exhaustion, Depersonalization, and Personal Accomplishment). There was a positive relationship between humor and Personal Accomplishment $(r=.36, p<.002)$. Subsequently, differences in burnout between community college nursing faculty with high humor usage were compared to those scoring low on humor usage. This revealed a statistically significant difference for Personal Accomplishment. There was no statistically significant difference for either Emotional Exhaustion or Depersonalization with respect to low or high humor usage.

Talbot (2000) concluded community nursing faculty experienced high to moderate levels of emotional exhaustion and depersonalization with low feelings of personal accomplishment. Lower levels of burnout were found in nursing faculty in comparison to postsecondary educators and medical practitioners for all three MBI subscales, and the use of humor as a coping mechanism by nursing faculty tends to be related to a higher sense of Personal Accomplishment (less burnout). "Humor may moderate the impact of stress on burnout by assisting people with high levels of stress to cope" (Talbot, p. 359).

Talbot (2000) also cited other research correlating burnout to management style, collegial support, and workload. Humor seems to promote job satisfaction and facilitate a comfortable working atmosphere. However, research in the area of nursing and burnout is limited. In the current study, Talbot examined the correlation among community college nursing faculty members and their use of humor to relieve stress to avoid burnout. She concluded that academic burnout was an emotional phenomenon associated "with high achievement in the academic role" (Talbot, p. 359). She cited the workload of community college nursing faculty members as being stressful in the clinical setting more so than in the classroom setting. The clinical setting required clinical teaching and supervision of not only the student but also care of the patient. 
Nugent, Bradshaw, and Kito (1999) determined the variables that influence teacher self-efficacy in faculty with five or fewer years of teaching experience. According to the researchers, an educational environment dominated by uncertainty, innovation, and ambiguity mandates creating a balance between celebrating what has been done well and developing and identifying strategies that will work in the future. Teacher self-efficacy is defined as the confidence or belief in one's ability to teach. Historically, graduate education focused on teaching and encouraged self-efficacy in new faculty. More recently, the emphasis in graduate education has shifted from preparing faculty to educate advanced practice nurses and nurse researchers. Although this shift in the focus of graduate education has been beneficial to prepare nurses to deliver care, the concern now is that new faculty are not prepared to assume the faculty role and will not feel confident in their teaching skills.

Self-efficacy is internally derived and measured. It is the belief or view held by the teacher that he or she has influence over students in a way that brings about learning, regardless of how unmotivated or difficult the students may be. The emergence of selfefficacy theory was developed in the theories of Bandura (1994). Based upon Bandura's response-outcome expectancies, self-efficacy is defined as the perceived ability to engage in certain behaviors (efficacy expectation) that are most likely to bring about the desired outcomes (outcome expectation) (Nugent et al., 1999, p. 230). Behaviors characterizing self-efficacy include persistence, sustaining tasks, and clearly conveying expectations to the learner.

Two factors comprise teacher efficacy. First, personal efficacy is the belief that one has the skills or personal ability to bring about a positive change to students. Nursing faculty members with clinical competence have a strong sense of personal efficacy. 
Second, teaching efficacy is the belief that students can benefit from the actual educational experience as developed and presented by the teacher, regardless of the influence (positive or negative) of outside sources, i.e., with high quality instruction, the students can learn. Instructors can do their job; high teaching efficacy can be reached (Ashton, 1985).

It is possible to hold different efficacy beliefs related to the two subconstructs. An individual could believe that some instructors can do the job (high teaching efficacy--these students can be reached). Or, often related to beliefs in deficit theory, the individual may perceive that "those" students--typically, poor, minority, special education, etc.--can't learn no matter who is the teacher (low teaching efficacy). Similarly, the individual could believe that while others can be successful (high teaching efficacy), he/she cannot (low personal efficacy) or that he/she can do the job (high personal efficacy) (Ashton, 1985).

Nugent et al. (1999) related Bandura's framework to the application of nursing education, because the nurse educator bridges the theory to practice gap. Faculty with a limited knowledge base in the process of education and with little teaching experience would have less of a sense of control over the teaching-learning environment and consequently would have a weaker sense of personal self-efficacy than might be seen in more knowledgeable and experienced faculty. On the other hand, their sense of personal efficacy is likely to be related to the type of students in their program. Nurse educators from a privileged, white background could observe the struggles of nursing students from low income or minority backgrounds and conclude that the problems are related to the students' backgrounds (they just don't have what it takes to become nurses--low teaching efficacy) rather than concluding that different and/or more effective strategies are needed for those who come to the program with less preparation for academic success. Effective instruction in nursing requires teachers to have high levels of both personal and teacher 
efficacy.

In their study, Nugent et al. (1999) examined four research questions: (a) how confident do new faculty feel in the teaching skill domains of course preparation, instructor behavior, evaluation and examination, and clinical teaching, (b) is there a difference in the perceived self-efficacy in faculty who reported having completed additional educational coursework, (c) is there a difference in perceived self-efficacy of faculty in relation to years of teaching experience, and (d) is there a difference in the perceived self-efficacy of faculty who reported having teaching experience in related areas?

The sample for the study consisted of National League for Nursing (NLN)accredited baccalaureate programs in which the dean or director reported having faculty who met eligibility criteria. Of the 535 deans and directors contacted, 303 responded. Fifty-six stated that faculty in their programs did not meet eligibility criteria, 12 declined participation; and 235 agreed to distribute questionnaires. A total of 1,352 questionnaires were mailed, 450 surveys were returned, 346 met the eligibility requirements and were included in the study sample (overall response rate $33 \%$, and usable response rate $25.6 \%$ ). The majority of the faculty were female (95.4\%), between the ages of 31 and 50 years $(82 \%)$, and master's prepared (83.5\%). Regarding faculty teaching experience, $26 \%$ reported five years, $20.8 \%$, two years. The majority $(59.5 \%)$ indicated having previous teaching experience in a field other than nursing (staff development, higher education). A majority of the faculty $(71.1 \%)$ of the faculty reported having some formal nursing education courses; 29\% reported none (Nugent et al., 1999).

The instrument used was the Self-Efficacy Toward Teaching Inventory (SETTI), plus a demographic data sheet. The SETTI is a 48 -item self-report scale measuring the degree to which subjects feel confident in their ability to execute specific teaching 
behaviors across four domains: course preparation, instructor behavior, evaluation and examination, and clinical skills. Participants responded to each statement using a Likerttype scale format ranging from "not confident" (1) to "completely confident" (4). The instrument was revised by the researchers to include 19 items that dealt with selecting teaching strategies, promoting critical thinking, and more specific behaviors associated with clinical teaching and evaluation of student performance in the clinical area. Faculty reviewed the modified version for content validity; the Cronbach's alpha was reported at 95. Descriptive analyses were computed on the demographic variables. To identify factors that influenced self-efficacy, the investigators examined relationships between demographic variables and the mean score on the SETTI. Correlational analysis, multiple regression, and analysis of variance were used to determine the relationships of the dependent variable (SETTI) with study variables. Factor analysis was performed on the investigator's version of the scale to confirm construct validity (Nugent et al., 1999).

To determine how confident new faculty felt in the teaching domains of course preparation, instructor behavior, evaluation and examination, and clinical teaching, a mean score was calculated for the total scale and each subscale. The mean scores (and standard deviation) for the subscales were: course preparation, 40.6 (3.36); instructor behavior, 47.4 (3.40); evaluation and examination, 34.7 (3.09); and clinical skills 48.0 (3.43). Correlation of the demographic variables of interest with the dependent measure demonstrated significant positive relationships with age $(r=.164, p<.01)$, hours of formal education courses $(r=.193, p<.01)$. A positive relationship was found between the dependent measure and the highest educational degree $(r=.130, p<.05)$. No significant relationship was found between faculty rank and years of non-nursing teaching and the SETTI scores. A multiple regression was used to ascertain the variance for the SETTI scores by age, 
highest education degree rank, formal education courses, teaching experience in nursing, and other teaching experience. Age accounted for $2 \%$ of the variance found in the SETTI scores (statistically significant at $p<.05$ ). Formal education courses, teaching experience in nursing, and other teaching experience accounted for $12.2 \%$ of the total variance. The number of years in teaching experience in nursing education accounted for $10 \%$ of the variance, the greatest amount (Nugent et al., 1999).

New nurse educators in this sample were confident in their teaching skills as documented by the reported mean scores of 3.09 to 3.43 on the four-point scale. The level of teacher efficacy (as defined by the SETTI subscales) was most likely influenced by the fact that in this sample the majority of participants had taught for 3 or more years in a nursing program and had taken formal nursing education courses. This did not support the investigator's belief that formal education courses influenced teacher efficacy more than other variables. New nurse educators scored the highest in the clinical skill domain (3.43). Nugent et al (1999) suggested that because the least confidence was in the areas of evaluation and examination, perhaps education courses in evaluation and examination would strengthen teacher self-efficacy. Orientation of new faculty should consist of instruction in the domains of course preparation, instructional behavior, and evaluation and examination, the three areas that nurses have least familiarity with compared to their strengths in clinical skills. Experienced faculty who can counsel, guide, and help a lessexperienced faculty member should mentor them. An increased feeling of self-efficacy will empower faculty to be creative in providing a learning environment that will successfully prepare students in a new health care environment. A weakness of the study is that it defined teacher efficacy in terms of only one of the two constructs inherent in Bandura's (1977) theory and subsequent work by other investigators (e.g., Ashton, 1985) on the 
distinction between personal self-efficacy (can I do the job) and teaching efficacy (can teaching make a difference, apart from my own personal ability).

Choudhry (1992) investigated the existence of a consensus among nursing faculty and their deans at universities and community colleges on the minimum acceptable competencies of newly appointed faculty involved in carrying out various sub roles. Very little research has been reported on essential competencies inherently necessary to perform the multiple roles of a nursing faculty member. Consequently, there is a lack of educational preparation precisely for, or socialization into, the nurse educator role. Most literature is focused on faculty teaching in university programs; no study had specifically addressed role issues concerning community college faculty. The role of the educator can be learned, through formal education preparation, informal learning from experience, the influence and observation of mentors, and continuing education. Using role theory as a framework, it was predicted that educational level and years of experience would have a positive relationship on the rating of competencies. Therefore, one of the purposes of the study was to test the hypothesized relationship of role theory to the rating of teaching competencies. The researcher believed that regardless of the type of institution (university or community college) or program (baccalaureate or associate degree), nurse educators are expected to perform a number of common roles with competency.

Choudry (1992) hypothesized that: (a) there would be no difference among the faculty respondents by type of institution in which they were employed as to the rating of competencies, (b) there would be no difference between responses of the faculty and those of deans on the rating of competencies, (c) the educational level of the faculty respondents bears no relationship to their rating of competencies, and (d) the length of the respondents' teaching experience bears no relationship to their rating of competencies. 
The questionnaire contained 96 competencies plus demographic information, educational background, and teaching experience. A five-point Likert-type scale was used with respondents selecting the number that best represented the degree of a competency from (1) least desirable to (5) most desirable. The rating of competencies was from ideal to beginning level. Respondents were asked to rate each competency at two levels: the level of competency a nurse educator should possess and the acceptable level of competency for a beginning nurse educator.

The questionnaire was reviewed for clarity and content, and a pilot test was conducted using one community college $(N=15)$ and two universities $(N=5)$. Cronbach's alpha for the instrument was greater than .88 for all sub-roles. The questionnaire had five sub-roles: teacher role, practice role, research role, service/governance role, and role for personal and professional growth. The study sample consisted of full-time faculty who taught $50 \%$ of the time in all of the community colleges, all full-time faculty of all the universities in Ontario, and all the heads of these institutions. Six hundred twenty-three questionnaires were mailed, 291 (46.7\%) were returned, and 268 could be used for the study. The final sample included 193 community college faculty, 60 university faculty, 4 university deans, and 11 community college deans/chairpersons. All respondents rated the 96 competencies desirable for a beginning faculty member. Mean ratings ranged from 2.71 to 4.63. Kendall's Coefficient of Concordance was computed for the rankings for both sets of faculty. The sub-roles of teaching, practice, research, and service $(W<90)$ showed complete agreement between the two sets of faculty. There was no agreement $(W=.48)$ on competencies for personal growth (Choudry, 1992).

With the alpha level set at .05, MANOVAs, were identified on each dependent variable to determine which of the variables were specifically significant. There was a 
significant difference with the overall ratings of competencies by type of institution, $F(14$, $251)=9.48, p<.001$. The significant differences arose from the ratings of the following competencies: evaluates students' performance (STUEVAL); facilitates students' clinical practice (STUCLIN); acts as a student advocate, advisor, and resource (ADVISOR); disseminates research to procure funds and advances nursing through research (ADVANSG); and has the ability to critique research and apply research in practice and teaching (CRITRES). Thus, the null Hypothesis 1 was rejected.

Results of the second hypothesis showed that deans and faculty expected the same level of competencies for a beginning faculty member. With the difference in their rating not significant, this null hypothesis was not rejected. The third hypothesis stated that respondent's educational level would influence the ratings assigned to the competencies. The MANOVA was significant for STUEVAL, ADVNSG and CRITRES. Faculty with a baccalaureate degree gave higher mean ratings than those with master's degrees for STUEVAL ( 4.25 vs. 4.04$)$. Faculty with a Doctorate rated research competencies higher than those with master's degrees and those with a baccalaureate degree rated them lowest. Thus, null hypothesis 3 was rejected. Finally, based on a correlational analysis, the relationship of length of teaching experience to rating of competencies was not significant; accordingly null hypothesis four was not rejected (Choudry, 1992).

In addition, Choudry (1992) analyzed the difference in ratings between beginning and ideal levels of teaching competencies. These ratings were not statistically significant. However, further analysis of the differences between the beginning and ideal measures showed that university and community college respondents had significantly different responses. The gap between the beginning and the ideal ratings was greater for the community college faculty ( 2.93 for beginning and 4.24 for ideal) compared with 
university faculty ( 3.90 for beginning and 4.74 for ideal) regarding the ability to critique research and to apply research to practice and teaching. On the other hand, the complete agreement by the two faculty groups, full-time faculty and the heads of faculty, on three teacher-role competencies indicated certain core competencies: the common educational and socialization needs of all new nursing appointments. These findings suggest that the socialization of the new faculty member needs to begin during educational programs prior to graduation as well as in the orientation process of the employing institution.

Fugate and Amey (2000) examined community college faculty in the early stages of their careers, the factors leading to their conceptualizations of their roles, and potential impact of this for faculty development. The review of literature included the lack of research on community colleges despite their continuous growth. The lack of studies involved with faculty models and the development of the role of the community college faculty member was cited as the reason for the study. The authors posed four research questions: (a) what career path did the recently hired full-time follow (b) how did they describe their roles during the early stages of their career (c) what was the stage of the changes during the early years of their career, and (d) what role would faculty development play in the early career? The design was a qualitative study with the use of interview tapes from face-to-face interviews with semi-structured protocols, transcripts, and field notes. Twenty-two early career college faculty at a Midwest community college were purposefully selected and met the criteria: (a) they were in their first six years of full-time teaching at a community college, (b) they represented diversity between liberal arts and the technical programs, and (c) they were represented by individuals of gender and cultural diversity.

The analysis of data and the development of emergent themes were guided by the 
works of Merriam, Lincoln and Guba, and Patton (cited in Fugate \& Amey, 2000). Data were collected and divided into three areas: (a) faculty career paths to the community college, (b) roles assumed by community college faculty during early stages, and (c) the impact of faculty development during early career stages. The vast majority of subjects in the study had not planned to become community college teachers. The factors which attracted them to the profession and led to retention were the opportunity to avoid the tenure process, the focus on teaching in the college setting, and the ability to teach without the doctoral degree. Some faculty were pleased with salary opportunities based upon the years of experience and academic degrees. Some faculty felt a sense of employment security in the community college setting and several had attended community colleges in the pursuit of their degree.

The roles of the faculty during the early career years were centered on teaching with the assistance of a mentor, role model, coach, or advocate. As faculty members moved into the second year, the expectations of community service became a reality. Focusing on the needs of the community was an important aspect of the faculty, and service within the institution through committee work was valued. Faculty were divided as to the expectation of research as part of the faculty role. Faculty subjects felt that the changes in their roles across time were most evident between the first and second year. The majority of the faculty related content in their roles and did not expect changes in the future. They did express some concern over burnout from teaching the same classes every year. Faculty development made a significant impact on the subjects in their early career development. Many cited the need for instructional assistance to develop teaching skills. The respondents valued faculty development. Continued professional development was seen as the responsibility of the faculty member and the role of the institution was to 
provide support. The views of liberal arts and technical-vocational faculty were similar, as were comparisons by gender and cultural ethnicity (Fugate \& Amey, 2000).

Several new faculty hired over the past six months expressed concern about the need to retain faculty through incentives. The discussion of faculty shortages was included as affecting faculty retention. Private industry and its incentives for faculty was a key component in the lack of retention of faculty. Fugate and Amy (2000) noted that the limitations of the study included the sample size and the need to examine other faculty early in their careers at other community colleges. A better understanding of the role of the labor market and job retention as well as faculty support efforts were seen as important for the future of the workforce.

Research examining nursing faculty orientation was conducted by Morin and Ashton (1998) to look at decreasing stress on new faculty by presenting programs which included "a sense of understanding of operating procedures, a better grasp of teaching responsibilities, and the establishment a network of colleagues" (Morin \& Ashton, 1998, p. 295). The authors reported that the experienced faculty member who leaves one setting to go to another is faced with the unknown and becomes stressed. Information provided in a nursing orientation is a topic that is lacking in the area of research. The movement of any nurse into another role creates stress in that person's life. Through a direct replication and extension study, an identification of the orientation protocols that exist for a seasoned graduate nurse faculty changing academic work environments was examined.

Role theory, from Biddle (cited in Morin \& Ashton, 1998), was utilized to address role overload and role strain and its influence on one's perception of accomplishment. A convenience sample of experienced faculty (three or more years of teaching) who had taught at the graduate level was identified. Subjects were chosen from 233 master's 
programs accredited by the National League of Nursing. Ninety four percent of the subjects were doctorally prepared. Five percent of the sample reported the failure to achieve tenure as a reason for the change to teaching; other responses for change were related to a desire for continued growth. The subjects completed an Experienced Faculty Orientation Questionnaire assessing the orientation offerings to graduate nurse faculty. The respondents were to evaluate, rank, and respond to a variety of questions dealing with: (a) academic environment, (b) educational environment, (c) social milieu, (d) political milieu, (e) geography, and (f) local nursing professional milieu. Determination of reliability and validity was reported. Subjects were placed in one of three groups depending upon days of orientation. Analysis of variance (ANOVA) was performed to determine statistical significance. Educational milieu was the only category in which the difference between groups was statistically significant $(p<.05)$. Other categories not statistically significant but rated highly were academic milieu, social milieu, and geography of the campus. Participants suggested the assignment of a mentor would be very helpful. Further research was suggested to examine the role of administrators in orientation.

Lia-Hoagberg (1982) assessed the progress of nurses with Doctorates in academe and compared their professional activities with academic women from other disciplines. The study participants were nurse and non-nurse faculty from eight Midwestern universities $(N=519)$. The sample consisted of three groups of faculty women: (a) nurse Doctorates (nurse faculty with doctoral degrees), (b) master's nurse faculty (nurse faculty with master's degrees), and (c) academic women (non-nurse faculty with doctoral degrees). The researcher attempted to select randomly an equal number of subjects for each group. The final sample of subjects who returned completed surveys ( $64.5 \%$ return rate) was (a) 129 nurse-Doctorates, (b) 116 nurse faculty with master's degrees, and (c) 90 
academic women.

The methodology included analysis of variance (ANOVA) of the grouped data obtained from the Biographical-Professional Activities Questionnaire (B-PA) and multivariate analysis of variance (MANOVA) of the data obtained from the Personality Research Form-Form E (PRF-E). The B-PA, designed by the researcher, included biographical information such as: (a) educational preparation, age, years since Doctorate, present academic rank, (b) current percent of employment, and (c) major field of academic work. Professional activities identified in the questionnaire included primary academic functions, percentage of time allotted to the academic functions, attendance at regional or national professional meetings, and numbers and types of publications and grants received outside of their institutions. The PRF-E contained 352 items with true-false responses, 20 need scales, and 2 validity scales. The scales included such items as: abasement, achievement affiliation, autonomy, change, cognitive structure, order, play, exhibition, and understanding. Validity coefficients ranged from .50 to .91 . A pretest was conducted with subjects who represented each of the groups at a four-year college within the state. Subjects completed the instruments and critiqued the materials (Lia-Hoagberg, 1982). One independent variable was biographical characteristics of faculty (field of Doctorate, educational level, years since Doctorate, and academic rank) and the dependent variable was professional activities (teaching, administration, research, consulting, own clinical practice, professional writing and publications, and grants). The second independent variable was educational level (nurse Doctorates, master's nurse faculty, and academic women) with the dependent variable being personality needs (order, play, sentience, social recognition, succorance, understanding, dominance, change, and autonomy). Lia-Hoagberg (1982) hypothesized: (a) women faculty nurses with doctoral 
degrees would exhibit higher levels of professional activities than women faculty members with master's degrees, (b) non-nurse faculty with doctoral degrees would exhibit significantly higher levels of professional activities than women nurse faculty with doctoral degrees, (c) there would be no significant differences in personality needs between women faculty nurses with doctoral degrees and women faculty with master's degrees, and (d) there would be no significant differences in personality needs between women faculty nurses with doctoral degrees and non-nurse faculty with doctoral degrees. Results indicated that the academic women showed a greater number of years (6-15 years) since earning the Doctorate (27.3\%) compared to 9.3 percent of the nurse Doctorates. Academic women and master's nurses tended to be younger in age than nurse Doctorates. Over $90 \%$ of the nurse Doctorates were between the ages of 36-65, while a greater majority of the nurse master's and academic women were in the 26-45 age range. Nurse Doctorates spent more time in administrative duties than the other two groups; $10.2 \%$ for academic women and 5.2\% for master's nurses. Post hoc pair-wise comparisons using Newman-Keuls analysis showed that significant differences were found in teaching time between the academic women and nurse Doctorates compared to the master's nurse group. The master's nurse group spent over $50 \%$ of their time in teaching whereas the academic women and the nurse Doctorates spent $37.5 \%$ and $37.2 \%$, respectively.

In the second part of the study, Lia-Hoagberg (1982) studied the personality characteristics in relation to the three groups. Academic women and nurse Doctorates were significantly higher on the need for autonomy than the master's nurse faculty. The nurse Doctorates also scored significantly higher on the needs for Change and Dominance than the academic women and the master's nurse group. The nurse Doctorate group and the nurse master's group were significantly higher on the needs of Nurturance, Order, and 
Play. Implications for the study included an aging workforce of nurse Doctorates with an expected retirement of the greatest majority of the women in nursing faculty positions within the next 10 years. The nurse Doctorates' group also scored higher on the need for change than the academic women's group. Lia-Hoagberg suggested that the need for change was a result of the rigid policies encountered in hospitals. Both groups of nursing faculty scored significantly higher than the academic women on the needs for nurturance, order, and play. Nurses are nurturers of clients and the need is embodied in the role of the nurse. Nurses may use play as an emotional and physical release from the stress and crisis situations experienced in the role of the nurse.

Siler and Kleiner (2001) conducted a phenomenological study to gain an understanding of what new faculty members experience early in their career. Phenomenology is "the study of being in the world" (Siler \& Kleiner, p. 398). Novice faculty were purposefully sampled from a group of volunteers who were in their first year of employment in their current position. There were 12 students in the sample who were volunteers from programs who were members of the American Association of Colleges and Schools. Twenty-four Deans or Directors of baccalaureate programs provided names of faculty who were in their first year of teaching. There were six experienced and six novice faculty in the sample. Each subject was interviewed either in person (12) or by phone (2). A hermaneutic analysis to gather information concerning an individual's experiences and practices, disclosed in dialogue, was used to uncover shared meanings. The meaning of the experiences for the new faculty surfaced after the recorded interviews were studied. Four themes were identified in the analysis: (a) expectations, (b) learning the game, (c) being mentored, and (c) fitting in. Siler and Kleiner focused on expectations for their research article. The new faculty shared a common theme of expectations. Reasons 
for making career moves, meeting new colleagues and proving themselves were other common themes.

The researchers observed how faculty approached unfamiliarity. Even though the new faculty members were new to academe, they were all experienced nurses. But their experiences in the healthcare setting did not prepare them for the academic preparation and the clinical demands necessary to understand the clinical instructor's role. Other areas that emerged were (a) assistance from colleagues, (b) trouble finding answers, (c) performance concerns (d) criticism, and (e) previous experience. The researchers compared novice faculty with novice nurses as identified by Benner (cited in Siler \& Kleiner, 2001).

A descriptive research study by Herrmann (1997) examined the relationship between preparation to teach nursing and the use of clinical instruction methods. Questionnaires were mailed to all baccalaureate NLN accredited nursing programs via the Dean of the nursing program. Each Dean of Nursing was to give the questionnaire to three subjects who met the criteria. One thousand three hundred and two subjects were in the initial phase. The findings were tallied from 692 questionnaires (52\% total). The researchers used an adapted version of the Clinical Teaching Self-Assessment (CTSAF) as well as demographic questions and information dealing with time spent in each clinical teaching technique; space was included for responses to written problems. The group was exclusively women in the age range of 26-65 years. Their employment responsibilities included both classroom and clinical supervision. Sixty-six percent of the subjects reported that they had taken a class in curriculum development during their master's preparation. Fourteen percent of the individuals reported no course work to prepare them for teaching.

The results showed that faculty were spending around $70 \%$ of their time in student related activities. Scores on role modeling at 6.2 hours was the next highest behavior. 
Faculty reported that they supervised from four to 28 students in the clinical setting. A chisquare analysis was used to determine if there was a relationship between the amount of education and the feeling of adequacy in preparation. The findings showed that as faculty increase their level of preparation to meet the needs of their teaching role, they reported significantly more confidence that they were better prepared to teach in the clinical setting. Faculty confirmed that courses in curriculum, teaching methods, and a teaching practicum were most helpful. The study did not reveal differences between faculty educational preparation and how prepared and confident they felt they were to do clinical teaching. But implications for future research include the revision of the questionnaire to ask more openended questions and essays on clinical teaching. It was also suggested that the comparison of student outcomes in relation to teaching methods would be important.

\section{Orientation of New Faculty}

Genrich and Pappas (1997) examined the development, implementation, and evaluation of a new nursing faculty orientation at a private university in the Southwest. Information available to faculty on the first orientation day at the college included attendance at a faculty meeting, introductions to faculty and support staff, and a tour of the facilities. Information to new candidates imparted in a group format and with an overall passive approach to learning could be very overwhelming to the new faculty member. That concentrated approach to orientation was in fact overwhelming, not conducive to content retention, and did not promote adequate future utilization of the written policy and procedure manual. Faculty felt intense need to prepare for the first day of class and the anxiety took precedence over orientation. An open-ended questionnaire seeking evaluation and suggestions for the existing orientation was sought from responses of faculty hired within the last three years. 
Genrich and Pappas (1997) related a second problem occurring when the changing composition of faculty created a shortage of nurses prepared as nurse educators. This necessitated the recruitment and hiring of professionally competent nurses who were inexperienced as teachers. Currently the trend in master's programs is to prepare students to pursue clinical specialization and practitioner roles, while doctoral programs emphasize the preparation of researchers. The scarcity of this critical resource has created a number of challenges for current nursing faculty and serves a threat to the quality of nursing education. It necessitates that the traditional approach used to orient and socialize new faculty into their role be evaluated and improved.

According to Genrich and Pappas (1997) a good orientation can decrease the turnover of new employees, helping them to feel comfortable and become more quickly competent in their jobs. The orientation was developed to address the perceived needs of faculty hired during the previous three years. A survey was distributed to these faculty members to determine what they liked and what they had expected in their orientation to the school. Strong support emerged for a more gradual introduction to the policies and procedures of the institution to decrease the feeling of being overwhelmed. The faculty unanimously related that a support system, usually in the form of a mentor, was the most valuable resource to facilitate orientation.

The orientation designs for the Genrich and Pappas (1997) study included both general and specific components to foster integration and meaningful support for new faculty, thus encouraging the use of teaching methodologies that would facilitate retention. The orientation consisted of three parts. The first was predominantly a general orientation which addressed new faculty needs in a timely and meaningful manner. Using a selfdirected approach provided information from most relevant to least and most immediate 
for job entry. New faculty were given the faculty handbook around six weeks prior to their starting date. The second part provided orientees with resources necessary to prepare for role implementation. One month before the first day, the orientee received a course syllabus and a letter with course textbooks and their availability. Many times faculty are hired within days of the beginning of classes. The third part asked faculty members to complete a self-assessment concerning previous teaching experience and various experiences and skills relevant to teaching in the classroom and clinical setting. The orientation provided an opportunity to give individualized information to each new faculty member. Knowledge gained from the orientation could also provide information to course leaders as to what to expect from new faculty in their department, as well as appropriate socialization of the new faculty member to the department.

As a second component, time was allocated on the first day of employment for clarification and discussion of the self-study material previously read. Follow-up meetings were designed to facilitate open discussion regarding any concerns or problems new faculty may experience. The meeting schedule was flexible and adjusted to the needs of the faculty. The third part of the study was conducted by the semester coordinator and was designed to facilitate integration of each new faculty member into their specific teaching assignment. The model included a checklist with topics such as course specific content, mentor availability, assignments, and student evaluation (Genrich \& Pappas, 1997).

The orientation plan was initiated in fall of 1993 for three faculty members. Two were experienced teachers of baccalaureate nursing students and the third was a clinical nurse specialist without academic teaching experience. Comments from the new faculty, coordinators, associate dean, and dean were solicited by means of a voluntary, individual interview at the end of the spring 1994 semester. New faculty members were asked to 
critique the model. Overall they reported that the availability of general orientation material before the onset of the semester was a helpful strategy that allowed them to prepare ahead of time. All three faculty believed that they were able to participate in the new faculty orientation with some knowledge of the institution and academic unit already in place (Genrich \& Pappas, 1997). Each new faculty identified a mentor as the most helpful resource they used. A mentor was identified as the person who could explain realistically what to expect with solutions and approaches to problems. It was suggested that the assigned mentor establish a regular meeting schedule to make access easier. Each faculty member, regardless of teaching experience, expressed the need for a mentor to provide initial and ongoing support. It was also suggested that the mentor have access to the self-assessment of the new faculty member to assist in further support.

The revision of the orientation process, according to Genrich and Pappas (1997) heightened awareness of how important effective communications was to a new faculty member's survival and success. The need to strengthen and formalize the mentor relationship was well supported by the new faculty. Access to a consistent, friendly, and experienced faculty member offered a sense of security and belonging to the organization for the orientee as they tried to readjust and modify behaviors consistent with the new faculty role.

Kavoosi, Elman, and Mauch (1995) conducted an interpretive descriptive study with correlational design to investigate the relationship between senior nursing faculty mentoring activities and support provided by nursing program administrators for faculty mentoring. The researchers emphasized the psychological functions of mentoring that affect the individual on a personal level, which focused on self-worth. In contrast, career functions promoted the individual's career advancement. Mentoring was found to have an 
impact on the mentor and the protégé and the working environment; it also was suggested that the environment had an impact on the development and quality of mentoring relationships. Environmental impact is believed to influence the formation and length of the relationship, its quality, and the mentoring activities provided. The three research questions asked were: (a) what mentoring activities do senior nursing faculty provide in NLN-accredited master's degree programs, (b) how do nursing program administrators support faculty mentoring activities and what level of organizational/institutional support do they identify, and (c) how does nursing administrative support for mentoring affect the mentoring activities of senior nursing faculty?

The developmental theory of Erik Erikson (cited in Kavoosi et al., 1995) was used for the purpose of the study since mentoring was seen as an adult developmental phenomenon. The researchers examined the stage of Intimacy vs. Isolation of the new, younger faculty member, and the stage of Generativity vs. Stagnation for the middle-aged adult. Data were obtained from two surveys, the Administrative Data Questionnaire (ADQ) (cited in Kavoosi et al.), and the Alleman Mentoring Scales Questionnaire (AMSQ) (cited in Kavoosi et al.). The population included two groups: administrators and senior faculty (full or associate tenured professors) in all 154 NLN-accredited master's degree nursing programs in the United States. Graduate level nursing faculty with earned Doctorates and higher academic rank, who were likely to be more experienced and have more power within the organization than nurses prepared at the masters level were selected. Eighty administrators responded with completed questionnaires for a $51 \%$ response rate. Of a possible sample of 849,417 responded for a $49 \%$ response rate. Twenty-five percent of the respondents $(n=96)$ reported that they were not engaged in mentoring and were not a part of the final sample. Seventy-five percent $(n=293)$ indicated that they had been or 
currently were functioning as mentors to junior faculty in the work setting and returned completed surveys. The Administrative Data Questionnaire (ADQ) developed by the researchers was a 20-item, self-administered mailed questionnaire designed to elicit demographic information about the administrator, the faculty, and the institution, as well as faculty mentoring relationships, and existing institutional and administrative support for senior-junior mentoring relationships. A pilot study was conducted to determine project feasibility and questionnaire reliability. The Alleman Mentoring Scales Questionnaire (AMSQ) Form A was completed by the nursing faculty to report actions toward their protégé. The AMSQ was composed of ten scales, with a Likert-type scale measuring mentor behaviors: (a) teach the job, (b) teach politics, (c) challenging tasks, (d) counseling, (e) career help, (f) demonstrated trust, (g) endorse acts, (h) views sponsor, (i) protect, and (j) friendship. Cronbach's Alpha for the overall set was 99 .

Descriptive statistics answered the first two research questions concerning mentoring activities of the senior faculty and administrative staff. Kavoosi et al. (1995) used the Kruskal-Wallis test to examine three independent variables and ten dependent variables. Formal support (FS), conceptual support (CS), and no support (NS) for mentoring by administrators were the independent variables while the faculty scores on the ten mentoring scales were the dependent variables. Qualitative responses on the ADQ were analyzed for themes relevant to the administrator's definition of mentoring and mechanisms of support for mentoring identified by nurse administrators. Faculty views of mentoring were grouped into three categories: outcomes of mentoring, process of mentoring, and nature of mentoring. Data from the AMSQ were used to identify the mentoring activities of senior faculty. Using a 1 to 5 scale with 1 designated as unlikely or seldom and 5 designated likely or often, each respondent indicated how often or how likely 
each event would occur. Nursing faculty mentors reported teaching the job as the most likely activity $(M=4.23)$. Respondents were least likely to provide friendship $(M=3.54)$ or protection $(M=3.55)$. When asked about the focus of faculty mentoring relationships, $91.5 \%$ of the administrators believed that mentoring in their school focused on developing career skills of protégés which included teaching, presentations, research, and grant acquisition. Over 50\% also indicated that these relationships had a personal development focus, which centered on establishing trust, providing counseling, protection, and friendship. Seventy percent of the administrators perceived that mentoring relationships were established through informal networks, $5 \%$ reported through formal networks and $16 \%$ through a combination of both formal and informal channels. Fifty-eight percent of the administrators stated that their school supports mentoring between senior and junior faculty, while $19 \%$ related that institutional support was in the developmental stage. Twenty percent said that their school of nursing had established formal faculty mentoring programs. Rewards for these activities varied; $62 \%$ related that nursing faculty were rewarded, but only $3.8 \%$ of the group who rewarded faculty did so with tenure promotion and salary. Finally, $48.7 \%$ were rewarded verbally or by written acknowledgement of award ceremonies.

Data on the level of organizational/institutional support for mentoring were determined by administrator responses to select questions on the ADQ. Based on that information, the schools were placed in one of three categories of support: (a) formal support (FS), (b) support for the concept (CS), and (c) non-support (NS). Fifteen schools were rated in the formal support, 30 schools in the support for concept, and 23 schools were in the NS group. Kavoosi et al. (1995) applied the Kruskal-Wallis analysis of variance to the average scores of the faculty on each of the 10 Allman Mentoring Scales in 
each separate level of support group. No significant differences among faculty in the three different support groups were found. A Kruskal-Wallis test was computed to determine whether different support groups utilized mechanisms of support. The unit of analysis was the average of the rank scores of administrators on the nine measures of support mechanisms for the three support groups. Significant differences were found between groups in (arranging formal and informal gatherings $\chi^{2}=0.037, p \leq .05$, and (b) funding for joint projects $\chi^{2}=0.0004, p \leq .01$. A post hoc analysis determined that administrators in the nonsupport group provided significantly less funding for joint projects than the administrators in the formal support and conceptual support groups.

Faculty mentors reported that they were quite likely to engage in mentoring activities that focused on career and personal development. The three activities most often engaged in by faulty mentors were (a) teaching about the job, (b) demonstrating trust and (c) sponsoring the faculty member (Kavoosi et al., 1995). Informality was supported in this study as most administrators responded that faculty-mentoring relationships in their organizations were established through informal networks. Faculty may mentor because they value mentoring as a professional obligation. The top three informal support mechanisms utilized by administrators were: (a) modeling mentoring behaviors, (b) oral and written communication, and (c) allowing for flexible time scheduling. The major outcome of the study found no significant statistical relationship between the level of administrative support for mentoring and the type and extent of mentoring activities reported by senior faculty. No statistical relationship was found between administrative support for mentoring and the mentoring activities of the nursing faculty; however, there was a significant relationship between levels of support and administrator support mechanisms. The primary limitation is that the study cannot be generalized to faculty and 
programs other than NLN-accredited Master of Science degree programs whose administrators and faculty participated in the study. One method of facilitating mentoring throughout the organization is to establish mentoring as a faculty role expectation. Mentoring could become part of the employee performance evaluation and used as one of many criteria in the hiring and retaining of nursing faculty.

\section{Theories of Career Choice}

Increasingly researchers have been exploring the dynamics of career trajectories and choice. Some of this work is explicitly theoretical while other studies are more descriptive.

LaRocco and Bruns (2006) examined the perceptions of educational professionals who had chosen to move to the academic ranks with attention to the responsibilities of a faculty member to the college and to their peers. In a qualitative study, the authors posed three research questions: (a) how do second career academics describe their entry into the academy and their work during the early years of their second careers, (b) how do second career academics describe their connections to and experiences with their university community, and (c) how do second career academics describe their relationships with their colleagues? The authors chose full time, tenure track career faculty in four year colleges or universities who had practiced at least three years in an education-related field before assuming an appointment in their college. All subjects recruited held the Doctor of Education or Philosophy and had been in their academic position between one and six years. Through a purposeful sample by the use of listservs and discussion, individuals who met the criteria were sent an interview protocol, a consent letter, and a demographic form via electronic submission. Once acknowledgement of their participation was received by electronic mail, an interview was scheduled with the intent of meeting the needs of the 
participant. Eleven interviews were conducted: seven from a face-to-face interview and four subjects via telephone. All of the eleven interviews were audio taped with interviews lasting from 45-75 minutes. Analysis was carried out by reviewing the audio recordings holistically and then dividing the transcripts into segments. Each researcher read and reread the transcripts in order to categorize the information into themes and patterns. All items were coded and analyzed and records of personal reactions to the subject were kept in order to maintain subjectivity. The demographic findings included four males and seven females in the study with participants ranging in age from 28-52 $(M=41)$. Participants had been in a variety of fields prior to the new position: (a) educational administration, (b) teaching in early childhood to middle school, (c) recreation and athletics, and (d) business. The range of time spent in their previous career was from 3-29 years with an average of 12 . All of the participants obtained a teaching job in a college department that prepared future educators. Eight of the participants noted that their appointment was nine months in length; the other three had appointments for 12 months.

LaRocco and Bruns (2006) identified three dimensions as part of the organizing framework in the presentation of their results: entry into academia and work life, participants' understanding of their university community, and perceptions of their relationships with their colleagues and administrators. According to the researchers, the participants were unsure about being prepared to teach, conduct research, or to publish. The subjects related that they had difficulty balancing their work life and home life. Only three participants felt confident in their ability to meet all of the responsibilities of their role in academe. In relation to conflicts in meeting all of the responsibilities of academe and home life, most subjects "reported problems related to completing tasks, maintaining health, and feeling a sense of accomplishment" (LaRocco \& Bruns, p. 632). All indicated 
that these concerns were unlike what they had experienced in their other career. There were five subjects (four males and one female) who indicated a feeling of independence and autonomy related to their work. Of the other subjects who were ambivalent about autonomy, one indicated that there were great expectations but little time to work creatively. The second dimension relating to understanding the university was not as divided. Most respondents felt they could access resources to meet their needs. It was noted that the group members were able to identify resources for teaching and research assistance, technology, and funds for professional development. There were two members of the group who had very few resources and expressed frustration at their opportunity to access needed resources. Faculty in this study reported that they had experienced at a minimum one supportive relationship that provided them assistance in their adjustment. Unfortunately, there were four subjects who reported that they did not receive support from department chairs or program coordinators. Although support came from colleagues, there was little support by many of the new faculty from direct supervisors. The study had a small population of respondents but the qualitative nature of the design provides insight into the information gleaned from the subjects. Nurses moving from clinical practice to faculty positions could possibly experience these same feelings.

Adams (1985) compared responses of baccalaureate educators with those of associate degree nursing faculty in public community colleges and universities in Kentucky using Holland's Theory of Vocational Career Choice. Holland (in Adams) studied vocational choice, work history, job changes, and occupational achievement. Individuals are categorized as one of six types: (a) realistic, (b) investigative, (c) artistic, (d) social, (e) enterprising, or (f) conventional (Adams, p. 12). Six environmental types are also identified: (a) realistic, (b) investigative, (c) artistic, (d) social, (e) enterprising, and (f) 
conventional. Holland's theory suggests that people seek environments that suit their skills and abilities, and the behavior of the individual is an interaction between his personality and his environment.

Full-time faculty from eight public universities and eleven public community colleges completed a questionnaire, the Strong Vocational Interest Blank as well as an investigator developed section to gather demographic data. Of the 258 faculty who received the questionnaire, 161 subjects met the conditions of the study. Demographically the two groups were quite similar: female, married and in the 25-34 age range. Basic education and the numbers of years practicing as a nurse were similar. There were differences in why the faculty chose to teach, their level of education or level aspired to, as well as different memberships in professional organizations and professional journals that the two groups read. The associate degree faculty had the highest mean score $(M=51.7)$ for Holland's Social personality pattern, characterized by "helping other persons, solve problems using social competencies, teaching ability, and flexible approaches; and value social and ethical activities" (Adams, 1985, p. 163). Faculty reported that they spent numerous hours in the classroom and clinical as well as engaged in community service. University faculty had the highest mean score for the Artistic personality pattern $(M=$ 52.9). Holland characterized this pattern as preferring "the manipulation of physical, verbal, or human materials to create art forms; solve problems through artistic competencies; value esthetic qualities; and prefer an environment dominated by expressive, independent, unconventional, and flexible ways of doing things" (Adams, p. 164). Adams attributed the differences to the orientation of the teaching of the groups. The associate degree group teaches in a curriculum that is technically oriented and more structured with a desire for social contact. The baccalaureate group teaches in a more 
complex and abstract curriculum with a desire for independent and innovative approaches. Results from both groups in relation to the Strong-Campbell Interest Inventory occupational scales inferred that neither group strongly identified with the role of College Professor, but were strongly identified with Registered Nurse. Adams reported that a mean of 45 was indicative of a strong identification with the role. The associate degree group scored $M=44.2$ with registered nurse and 37.5 for college professor. For the baccalaureate group, the scores were College Professor $(M=41.9)$ and Registered Nurse $(M=48.4)$. Neither group was strongly identified with the role of college professor. Adams found that members of the two groups were not all prepared at the doctoral level and since all were registered nurses before taking positions as faculty, their perceptions of the role of the registered nurse would be the strongest. Fifty one percent of the community college faculty reported accepting their teaching job because it was offered to them; however, $49 \%$ of the baccalaureate group reported making the choice to teach as part of their career goals.

\section{A Sociopsychological Model of Career Choice}

Helen Astin (1984), a counseling psychologist, proposed a model in which the occupational choice of women is affected by social experiences and opportunity. Although focusing on women in career choice, she also noted that her model had application for both sexes. Exploring the role opportunity provided in career choice as well as the state of the economy and the condition of the job market also was important. The model incorporates four constructs: (a) motivation, (b) expectations, (c) sex-role socialization, and (d) structure of opportunity (Astin, p. 119). Work motivation and expectations were grouped as psychological factors. Sex-role socialization and structure of opportunity were identified as cultural-environmental factors.

1. Work behavior is motivated activity intended to satisfy three basic needs: 
survival, pleasure, and contribution.

2. Career choices are based upon expectations concerning the accessibility of alternative forms of work and their relative capacity to satisfy the three basic needs.

3. Expectations are shaped in part by early socialization though family, childhood play, school experiences, and early work experiences, and in part by the perceived structure of opportunity.

4. Expectations developed through socialization and through early perceptions of the structure of opportunity can be modified by changes in the structure of opportunity and this modification in expectations can lead to changes in career choice and work behavior. (Astin, p. 119)

Survival needs are those that ensure physiological survival. Through the action of work, the ability to provide food and shelter is tantamount to the survival of the individual and family. Pleasure needs refer to the action of work and the performance of meaningful activities in the accomplishment of goals. Contribution of needs is derived from the individual's sense of self-worth and self-esteem. "Survival needs are satisfied by means of the money earned through employment; pleasure comes from engaging in the work activity; and contribution needs are satisfied through the knowledge that the work benefits others" (Astin, 1984, p. 120).

In Astin's (1984) work on expectations, career choice, and work behavior, she examined what work options are available to the individual, male or female, as well as what options the individual is capable of performing. Expectations of the individual are a "function" of sex-role socialization and of the perceived structure of opportunity (p. 121). Sex-Role Socialization begins early in the child's life and is determined by how the male or female child is socialized within the family as well as society. The Structure of Opportunity is affected by the socialization of the individual. "The socialization process probably sets limits to changes in the structure of opportunity, whereas the structure of opportunity ultimately influences the values that are transmitted through the socialization process" (p. 122). 
Astin (1984) noted that about half of the working women in the 1970 s were more inclined to choose more traditional careers most commonly attributed to females (teaching, nursing, and other allied health fields). By 1982, female students entering college were less inclined to enter the more traditional female fields (45\%) and $28 \%$ were choosing careers in engineering, business, law, and scientific research. She also recognized that during the period of time in which she envisioned her theory, the $1980 \mathrm{~s}$, birth control provided a greater freedom for women to plan employment and childbearing, increasing advancement of women in their life's work. The traditional family in the period of Astin's theory development included the traditional family of the working husband and the wife and mother at home caring for children. Astin suggested that additional research regarding satisfaction of the three needs--survival, pleasure, and contribution in relation to a particular career choice at a particular point in time--would be useful in advancing the understanding of individuals and their work.

Lindholm (2004) interviewed faculty to understand their reason for choosing a career in academia. Using Helen Astin's need-based socio-psychological model of career choice (1984), the author analyzed topics that were identified and fit within the model. Astin's model (in Lindholm) translates psychological factors, work motivation, and expectations from cultural environmental as socialization and structure of opportunity. The model incorporates four central constructs: (a) motivation, (b) socialization, (c) the structure of opportunity, and, ultimately, (d) work behavior.

Lindholm (2004) examined the faculty member's personal attraction to faculty work, when the decision was made to pursue a career in academe, and who and/or what were influential in shaping the decision. There were 36 professors ( 12 women and 24 men) who were full-time, in tenure track positions teaching in one of four departments in the 
College of Letters and Science at a large public university. A random sample of faculty within each rank and gender was invited to participate using the college electronic means for communication. Each participant contacted received information on the study and the author's assurance of maintaining anonymity. If a prospective subject declined to participate, another individual was randomly selected who met the same rank and gender as the original subject.

Individual, semi-structured interviews were conducted in private offices or locations selected by the participant. An interview format was used to guide the discussion of the issues with five questions being asked of all participants: (a) How is it that you came to work at this institution? (b) What attracted you to pursuing a faculty career? (c) When did you decide that you wanted to be a professor? (d) Were there particular people who were influential in shaping your career decision? If so, what effect did they have? and (e) Did particular incident(s) or experiences influence your career decision? If so, how (Lindholm, 2004, p. 632)? The data obtained from each interview were audio-taped, then transcribed and content-analyzed for the identification of common patterns or themes.

An inherent attraction to academic work was identified as the participants spoke "passionately about their work" (Lindholm, 2004, p. 611). They reported their personal need for autonomy, independence, and individual expression as well as the overall satisfaction of being in the university environment. The freedom to engage in professional activities as well as engaging in intellectual pursuits was especially valued by faculty who were tenured. One quarter of those interviewed described their enticement to an academic career as one referred to as "a true calling" (Lindholm, p. 612).

The subjects' early experiences and family influences shaping their academic interests were identified by childhood experiences, undergraduate and graduate school 
training, and personal perceptions of competence. Participants were either from academic families (a member of the family was an educator) or had particular experiences with professors while in their programs, either undergraduate or graduate. Two thirds of the interviewed subjects reported that their decision to teach was from their undergraduate experience. The experience was either with a subject area that was extremely exciting to the participant or from an interaction with faculty members within the department who were especially supportive. Personal competence relating to the field knowledge and their general academic skills provided for the decision for some to choose teaching (Lindholm, 2004).

One third of the group interviewed expressed the idea that they accidently entered the field of teaching; they had no defined path to the role of academe. The group who characterized this lack of career path were all men, many who graduated from their program during a tight labor market and who accepted other non-academic positions only to realize that the position was not for them. The author also identified variations in the "socio-cultural factors" that defined the entry into the academic profession. "Faculty across the disciplines who completed their doctoral degrees and entered the academic labor market in the 1960s relayed a general sense of enthusiasm about the job search process and their ultimate acceptance of employment at this particular university" (Lindholm, 2004, p. 621). The author noted the tremendous job growth in academe and large numbers of educators hired during this period. Meanwhile, there was also an increasing interest in current political issues. These educators" "paths to this particular institution were marked not only by a passion for research and teaching, but also by an often compelling desire to impact social change" (Lindholm, p. 622). The younger faculty interviewed in the study were less politically motivated and by entering during a tighter job market, were more 
appreciative of the opportunity to teach in the only institution where they were offered a position.

Senior women interviewed spoke of the struggle of being unique to the group of faculty. Since very few women were hired in the early $60 \mathrm{~s}$, many recounted the struggle of career choice and experiences in the profession. Younger women stressed the struggles of maintaining competence and continued persistence in reaching their goals. When comparing the results to Astin's career choice model, the categories of survival, pleasure, and contribution were identified by the respondents. The need for pleasure was identified through the participants' verbalization of their passion for their role in education. Their desire to look critically at social issues and to challenge current thinking led some to characterize their work as pleasurable and they enjoyed the components of reading, writing, and teaching as part of their work. Survival needs were identified when the participants verbalized their understanding of the reality that a great deal of wealth would not be attained in their role as faculty but that it was adequate for their survival. Many related that the aspect of tenure provided significance to the role. The need for contribution was highlighted in various ways as a reason for pursuing faculty work. The opportunity to complete research which provided additional information for their field of interest was a significant point included in conversations. Also, the opportunity to contribute to social change through service-oriented projects and involvement in institutional activities was a common topic with women and minority faculty (Lindholm, 2004).

It was also highlighted from the study that although it was not identified as a part of Astin's model, participants identified a sense of creativity and self-expression as a faculty need. Participants also cited the ability to re-engage themselves continually in their careers in academe by venturing into new research areas or developing new programs or 
courses. Lindholm (2004) pointed out the results of her study reflect the importance of graduate education and professors' encouragement on the prospective faculty member. In many cases of faculty who chose to teach, a graduate faculty member had a great deal of influence on their decision to teach.

\section{Summary}

There is a critical shortage of nurses to meet the healthcare needs of the public. The employment picture does not get much brighter as projections for nursing positions continue to grow despite the recession. Factors affecting the increased need for nurses includes other employment options for women in today's workforce, the graying of the nursing workforce resulting in increased numbers of nurses retiring without the equal numbers of new nurses taking their places, and although there are an increased number of men and minorities applying to nursing programs, there are not enough to equal the need.

Compounding the situation of the nursing shortage is a lack of nursing faculty available to teach the increased numbers of nurses needed. Educational and work requirements are barriers to nurses taking faculty positions. When comparing the age of the faculty workforce with their clinical cohorts, nursing faculty are older and are more likely to have more education. Additionally, the need for advanced nurse practitioners resulted in colleges moving to the development of programs to educate the experienced nurse on the master's level as advanced nurse practitioners rather than utilizing masters programs to educate nurse faculty members. Salaries for nurses in clinical settings are higher than those in academe, and despite the demand in education for nurses with advanced degrees, there continues to be a gap between the clinical professional and the faculty member. Colleges experienced the decline in federal and state funding for lines that were vacated by faculty positions but not filled. This has left nursing programs and universities with the inability to 
increase their enrollments (not enough faculty to serve the additional student nurses).

Nursing education began with Florence Nightingale assisting in the care of war casualties and the realization that teaching hygienic measures was an important part in the care of the patient. Although the reputation of the caregiver was not favorable, the desire to care for others was the most important asset. Hospital-based programs provided the basic education for a considerable period of time. Student nurses would provide the staffing for the hospital and were supervised by upper level nursing students. Theory lectures were provided by doctors. To enhance the quality of nursing, collegiate programs were opened to provide a broader base of education. Nursing faculty began teaching students in the collegial setting, but the hospital-based programs continued to be the hallmark of educating nurses. As the demand for nurses increased, two-year nursing programs in community colleges were opened to increase the numbers of nurses in a shorter length of time while continuing to provide safe and efficient caregivers. These two year programs supplemented the four-year bachelor's programs in nursing education, even as the hospital-based programs were being phased out. As programs expanded to collegiate settings and advanced nursing programs opened, finding nursing faculty with advanced degrees was both crucial to the education of the nurse and a continuing barrier to expanded enrollments.

Research on recruitment of men and women to nursing has been important to determine what appeals to nursing faculty and how to reinforce those characteristics for future candidates. Insights into the recruitment of faculty in non-nursing positions can provide information that can be adapted to the attraction of nurses with advanced education to nursing education positions.

Retention of the current nursing faculty is paramount to any nursing program. Studies on job satisfaction report that there are differences according to the timing of the 
research and the college settings; however, independence in the activities of the job, support from colleagues, being an experienced leader, and having freedom within the department are important factors valued by nurse educators. Salary and work environment also play an important part in job satisfaction. Nursing faculty are experiencing job conflict and role strain as the demands for supervision of students in a continually changing healthcare environment impinge on the responsibilities of the faculty member in academe. Mentoring and a comprehensive orientation by colleges and nursing departments can provide opportunities for new nursing faculty to adapt to the roles and responsibilities of the college setting.

Theories that address career choice provide insight into the decision by professionals to choose nursing and nursing education. Age of the faculty member at the time of acceptance of the appointment as well as the condition of the current job market provide differences in nurses' decisions. Personality patterns could reflect career choice based upon the level of educational program in which the nurses choose to teach.

Nursing education has been affected by the events that have created the nursing shortage. It is absolutely necessary to maintain the role of the nurse educator for now and for the future. Through research on career choice, better understanding of how to recruit new faculty and retain both young and older nurse educators is possible. With the nursing faculty shortage looming ever larger, more needs to be done in recruitment of the brightest, most caring nurses to guide future generations of nurses. Among other promising directions for the future are orientation programs and mentoring. 


\title{
CHAPTER III
}

\section{METHODOLOGY}

\author{
Introduction
}

Past research in nursing education has explored the recruitment, retention, satisfaction level, and burnout of nursing faculty. Quantitative studies on mentoring emphasize the importance of providing support for the clinical nurse who joins the faculty ranks. Adams (1985), in a quantitative study, explored career choice using Holland's Theory applied to nursing faculty. Lindholm (2004) used a qualitative study to examine Astin's Model of career choice in relation to university faculty members. Lindholm stated that:

While quantitative approaches to vocational choice research can inform us of how prevalent certain experiences, perceptions, or personal characteristics are within a given profession, they are less informative for providing as to how, and why, people make the choices they do. Qualitative approaches enable us to understand more fully both the processes by which career decisions are formed and the motivations that underlie people's differential attractions to various vocational pursuits. (p. 607)

This chapter provides a description of the research methods used in the study including type of study (basic qualitative interpretive investigation and the role of the researcher in the study), description of the sample and population, procedures for the pilot study and data collection as well as the issues of trustworthiness, validity, and ethics. Using Astin's theory of career choice and a qualitative method, the overall central research question that guides the study was, "What factors affect the nurse educator's decision to teach?" 


\section{Research Design}

Macnee and McCabe (2008) define the qualitative method of research as focusing on the human context of life and its complexity. "Qualitative data analysis requires understanding, digesting, synthesizing, conceptualizing, and reconceptualizing description of feelings, behaviors, experiences, and ideas" (p. 28). The role of the researcher in qualitative research is that of observer of the phenomenon. By being able to observe the emic (the participant's view) while maintaining the role of the researcher as an outsider (etic perspective), the researcher is able to record the data as well as conceptualize and order the findings (Gall et al., 1996). Creswell (1998) defines qualitative research as "an inquiry process of understanding based on distinct methodological traditions of inquiry that explore a social or human problem. The researcher builds a complex, holistic picture, analyzes words, reports detailed views of informants, and conducts the study in a natural setting" (p. 17).

In the selection of the qualitative design, Creswell (1998) suggests that the nature of the question, how or what, determines the direction for the researcher to pursue. If the topic needs exploration, rather than the identification of variables, then a qualitative study is appropriate. When it is necessary to present a "detailed view of the topic," a qualitative study is the appropriate design, "This involves getting out to the setting or field of study, gaining access, and gathering material. If participants are removed from their setting, it leads to contrived findings that are out of context" (Creswell, p. 17).

For this study specifically, using a qualitative research design to collect demographic data and interviews from 20 associate degree nursing faculty members from the 20 colleges allowed the researcher to collect thoughts, feelings, and reflections in relation to the decision making process of career choice. Given the lack of studies that have 
addressed career choice qualitatively, these insights should add to the understanding of this subfield.

The interview is used in qualitative research to elicit information from respondents in their own words, which allows for an in-depth exploration of topics (Vierra, Pollack, \& Golez, 1998). Both questionnaire and interview methods have advantages and disadvantages. A questionnaire can be utilized to collect large amounts of data, takes less time to complete, and is far less the expense for larger samples, but cannot reveal the opinions and feelings of the subjects (Gall et al., 1996). Questionnaires also provide anonymity to the subjects and eliminate bias on the part of the interviewer (Polit \& Hungler, 1983). In contrast, interviews allow the researcher to probe for sentiments, deep seated values, or focused cognitions, allowing for the subjects to reveal dispositions not possible under the constraints of a questionnaire. Interviews are composed of oral questions to which subjects respond and can be face to face or utilize telephones or other technology. Subjects speak in their own words and the interviewer records their responses via audiotape or videotape, and typically supplements this with handwritten notes. The interviewer maintains control of the session, but schedules the time and location of the interview within the time constraints of the subjects. Of the three types of interviews-structured, semi-structured, and unstructured--this study employs face to face semistructured format. A series of set questions (the interview schedule) is asked, followed by probes if additional information beyond the original response is warranted (Gall et al.). Merriam (2002) cites four characteristics of qualitative research design. The first is that the researcher in a qualitative study seeks to understand the meaning of people and how they view their world and their experiences. Second, the researcher is the primary instrument collecting and analyzing data. By being able to observe verbal and nonverbal 
communication, the researcher is able to probe or clarify the responses within the setting. In light of this opportunity, the bias of the researcher can impact the study. "Rather than trying to eliminate the biases or 'subjectivities,' it is important to identify and monitor them as to how they may be shaping the collection and interpretation of data" (p. 5). Another characteristic of a qualitative design is that the process is inductive. This is truly the case when there is a lack of theory. "In attempting to understand the meaning a phenomenon has for those involved, qualitative researchers build toward theory from observations and intuitive understandings gleaned from being in the field" (p. 5). The final characteristic is that the results of the qualitative inquiry are "richly descriptive." The researcher uses words and pictures to support the findings. The quotes and excerpts contribute to the insights of qualitative research.

\section{Types of Qualitative Research}

Creswell (1998) identified five different approaches to qualitative research: (a) biographical life history, (b) phenomenological, (c) grounded theory, (d) ethnographic, and (e) case study. The focus for each of the approaches varies. In the biographical research tradition the focus is an exploration of the life of the individual at a certain point in time. Biographical focuses on exploring the life of an individual. Stories of the individual are collected and organized around themes that are pertinent to the subject's life (epiphanies). The researcher seeks to define meanings to the stories and may rely on the subject to help explain the meaning of the stories.

The focus for a phenomenological study is to develop an understanding of the essence of experiences about a phenomenon (Creswell, 1988). Interviews provide the data and the researcher details these in writing the phenomenon. A grounded theory study focuses on the development of a theory grounded in data from the field. Interviews of 20 to 
30 individuals provide a representation of the categories for formulation of a theory. Careful coding of the interview responses lends itself to the development or visualization of a theory which can be subjected subsequently to further empirical testing. Ethnography focuses on a cultural or social group of subjects providing a description or interpretation of the meanings of the behavior, language, and interactions of the group. Finally, the case study develops an in-depth analysis of a single case or multiple cases using multiple sources of data such as documents, interviews, and observations (Creswell, pp. 47-65).

Merriam (2002) included two additional types of qualitative research, basic interpretive qualitative study and postmodern research. The format of basic interpretive qualitative study focuses on the researcher who seeks to understand participants' thoughts and beliefs about a phenomenon, a process of capturing:

the perspectives and worldviews of the people involved....Data are collected through interviews, observations, or document analysis. These data are inductively analyzed to identify the recurring patterns or common themes that cut across the data. A rich, descriptive account of the findings is presented and discussed, using references to the literature that framed the study in the first place. (Merriam, pp. 67)

Postmodern research is the newest in formats and challenges the form and categories of the other traditional forms of qualitative research. It does not follow the ordinary format of any of the other type, and data analysis and reporting are unique to the each study. "Instead, postmodern research is highly experimental, playful, and creative, and no two postmodern studies look alike" (Merriam, p. 375).

The focus of the current research reflected the interpretive perspective. Through the interview process, the thoughts and feelings of nursing faculty educators in associate degree programs in Kentucky were captured. By detailing rich descriptions of the interviewees' responses, and comparing those results to the previous studies cited in the 
literature and the framework of Astin (1984), insight into the nurse faculty career choice will contribute to the body of research on career choice for nursing faculty.

\section{The Role of the Researcher}

In qualitative research the researcher plays a role by interpreting interactions with the subjects and how they function within the setting. Vierra et al. (1998) note that qualitative researchers have "positionality," i.e., they are in position to influence the research. In quantitative research designs the assigned role of the analyst is limited, whereas in qualitative designs the complexity of the role can be as a participant or nonparticipant observer. Participant observers "are actively engaged in the life of the setting they study. In addition to observing, they perform functions and enact roles that are meaningful to the people in the setting" (p. 200). The experience within the setting becomes part of the data they collect. This allows the researcher to be accepted as part of the group. Role conflict can occur when the observer confuses the role of researcher and participant. In the nonparticipant role, the researcher gathers the data through direct observation or by interviewing the subjects. Burns and Grove (2005) noted that the personality of the researcher is the "key factor in qualitative research....It is necessary for the researcher to be open to the perceptions of the participants rather than attach his or her own meaning to the experience" (p. 536). Observers in a qualitative study are the "instruments of observation, they often interact with people they observe and react to events as human beings in a real place. The human tendency to empathize--to put ourselves in the place of another--reinforces a subjective relationship with participants in the research setting" (Vierra et al., p. 212).

The broad functions of qualitative research designs are to increase understanding, promote participation or immersion, and link ideas and concepts (Macnee \& McCabe, 
2008). This study used the basic interpretive qualitative design with the interview format to produce a richly descriptive account of the findings.

From the review of the literature, this study seeks to develop an understanding of the career choice that associate degree nursing faculty make in deciding to teach nursing students in Kentucky. Nursing faculty members meeting selected criteria completed a short background survey, as well as participated in individual semi-structured interviews. The interview survey was reviewed by an expert panel to ascertain face validity. It is important for the investigator to probe for further information or clarification if the responses are not adequate to answer the original question. The data collected (transcripts of tape recordings, notes taken during interviews, and completed questionnaires) was read and reread. "Tapes become more than words; they contain feelings, emphasis, and nonverbal communications. The aspects are at least as important to the communication as the words" (Burns \& Grove, 2005 , p. 548).

\section{Emic and Etic Perspectives}

In qualitative research the investigator seeks to understand the complex phenomenon experienced by the subjects. The goal is to view the situation as the participants view it (Gall et al., 1996). To organize the data the investigator uses the emic perspective, the insider's view. The researcher then subjects this information to etic interpretation, that is, how to reconcile the responses of the subjects with the observer's understanding of the phenomenon, including cross cultural and scientific explanations (Vierra et al., 1998). "Qualitative researchers tend to have an emic emphasis in collecting data, because the goal is usually to represent the situation from an outsider's point of view" (p. 235). The emic perspective explains what an event means to the individual participant. Likewise, the etic construct refers to the researcher and what the events mean, how 
personal and cultural experiences, scientific training, and previous research influence the final interpretation of the data. Vierra et al. (1998) note that:

Etic constructs are employed in the design of the project and in the interpretation of results. Etic explanations belong to a larger universe than do emic constructs... [while emic] constructs enable researchers to understand how people within an organization perceive their world, and may help researchers explain those perceptions to other people. (p. 237)

Not all qualitative researchers give credence to etic perspectives, yet the very nature of interpreting the data collected is necessarily and inherently etic, whether the investigator acknowledges this or not. Accordingly, researchers should make explicit these influences rather than ignoring them or presuming that they do not exist (Miller, 2007; Danquah \& Miller, 2007).

Through the interview process associate degree faculty members will shared the individual emic perspectives of their career choice. With the accumulation of all of the responses, the researcher will coded and categorized the data. Interpretation reflected the personal realities of these faculty, as filtered through etic perspectives on differences across subjects, previous research, and the investigator's own understanding of the larger phenomenon.

\section{Population and Sample}

For this study, the target population included all associate degree nursing faculty teaching in community colleges and public university settings in Kentucky. This represents 20 programs; of these 16 represent community or technical college locations in the Kentucky Community and Technical College System (KCTCS), while four are associated with public universities. According to Macnee and McCabe (2008, p. 139), “The appropriateness and focus of sampling in a qualitative study both are driven by and drive data collection, and the detail and complexity of the resulting themes and theory will 
reflect the sampling." The sample should be representative of the population.

The sample size in a qualitative study is of suitable size if there is adequate depth and richness of the data collected. To ensure an adequate sample, the use of a purposeful method is appropriate. A purposeful sampling method provides the opportunity for the researcher to select specific subjects from the target population to meet the criteria being studied (Burns \& Grove, 2005). In this study, one faculty member from each of the 20 associate degree nursing programs in Kentucky were interviewed. These twenty subjects were selected using the following criteria: years of experience $(0-5,6-10,11+)$, educational attainment (BSN, working on MSN, MSN, working on $\mathrm{EdD} / \mathrm{PhD} / \mathrm{DNP}$, and $\mathrm{EdD} / \mathrm{PhD} / \mathrm{DNP})$, the Carnegie Foundation classification of all higher education institutions in profiles that include enrollment, size, and setting. The classification of setting defines small as enrollment less than 2,500 , medium as 2,500 through 7,500 , and large as greater than 7,500. The latest figures are based upon the 2003-2004 Integrated Postsecondary Education Data System (IPEDS) from the United States Department of Education Institute of Education Sciences. For the current sample, there are four colleges (two 4-year, two community colleges) classified as large in size. Eight colleges (one 4-year, seven community) are classified as medium, and eight (one 4-year, seven community colleges) are classified as small in size. The dichotomy adopted in Figure 1 results in eight programs classified as "Small" and 12 as "Medium/Large."

The purposeful sampling grid identifies the factors used in obtaining the 20 subjects to meet the criteria of the study. Education of the subject is identified as having attained E1 (BSN), E2 (Working on MSN), E3 (MSN), E4 (Working on EdD/PhD/DNP), and $\mathrm{E} 5(\mathrm{EdD} / \mathrm{PhD} / \mathrm{DNP})$. Across the top of the table, years of experience in teaching is designated as $0-5,6-10$, and more than 11 years. Included under experience is the size of 
the college where the subject teaches: Small or Medium/Large. A subject representative of the identifiers is notated with $\mathrm{X}$.

Table 1

Purposeful Sampling Grid Across Three Factors

\begin{tabular}{|c|c|c|c|c|c|c|}
\hline \multirow[b]{3}{*}{ Education } & \multirow{2}{*}{\multicolumn{2}{|c|}{$\begin{array}{c}\text { Experience } \\
0-5 \text { Years }\end{array}$}} & \multirow{2}{*}{\multicolumn{2}{|c|}{$\begin{array}{l}\text { Experience } \\
6-10 \text { Years }\end{array}$}} & \multirow{2}{*}{\multicolumn{2}{|c|}{$\begin{array}{l}\text { Experience } \\
11+\text { Years }\end{array}$}} \\
\hline & & & & & & \\
\hline & Small & Medium/Large & Small & Medium/Large & Small & Medium/Large \\
\hline E1 & $\mathrm{X}$ & $\mathrm{X}$ & & & & \\
\hline E2 & $\mathrm{X}$ & $\mathrm{X}$ & & & & \\
\hline E3 & $\mathrm{X}$ & $\mathrm{X}$ & $\mathrm{X}$ & $\mathrm{X}$ & $\mathrm{X}$ & $\mathrm{X}$ \\
\hline E4 & & $\mathrm{X}$ & $\mathrm{X}$ & $\mathrm{X}$ & $\mathrm{X}$ & $\mathrm{X}$ \\
\hline E5 & & $\mathrm{X}$ & $\mathrm{X}$ & $\mathrm{X}$ & $\mathrm{X}$ & $X$ \\
\hline
\end{tabular}

\section{Research Questions}

The research questions guiding this study are introduced in Chapter I and are included in this section for convenience of the reader. These guided the construction of the Background Questionnaire for Nurse Educators and Nurse Educator Interview Schedule (Appendix A).

1. To what extent are personal and demographic factors influential: 

a. Age?
b. Marital status?
c. Children or other family responsibilities?
d. Years of nursing experience?
e. Years of nursing faculty experience?
f. Family educational attainment?
g. Educational Attainment?
h. Influential person or event?
i. Size of college--Small or Medium/Large
j. Other?

2. In what ways do educational degree requirements for faculty positions:
a. Influence decisions to become a faculty member?
b. Influence career trajectory (tenure and promotion) and retention?

3. To what extent are working conditions in academia (as opposed to clinical nursing) influential:
a. Flexibility?
b. Collegiality?
c. Organizational climate?
d. Prestige/status?
e. Other?

4. To what extent are pedagogical considerations influential:
a. Love of teaching?
b. Lifelong learning?
c. Possibility of influencing the next generation of nurses? 
d. Content knowledge and skills?

e. Other?

5. What are the expectations for "being nursing faculty" when first hired regarding:
a. Teaching load?
b. Service?
c. Scholarly writing and research?
d. Earning tenure and/or promotion?

Beyond specific issues already addressed:

6. What are the primary barriers to becoming a nurse educator?

7. What are the primary satisfactions of working as a nurse educator?

\section{Instrument Development}

In a qualitative research design there are four primary methods of obtaining data: interviews, surveys, observations, and review of documents pertinent to what is being studied. In this study the Background Questionnaire for Nurse Educators was developed to obtain demographic data (see Appendix B). All participants were asked to complete the questionnaire immediately before the interview. The use of the questionnaire obtains the same information from all participants. Gall et al. (1996) noted that the respondent is in control of this method and can determine the amount of time needed as well as the order of completing the questions. However, not all information can be obtained from a questionnaire since it limits the ability to seek in-depth meaning to the thoughts and feelings of the participants. An advantage to the interview process is its adaptability to probe deeper into the responses of the participants and ask for clarification of responses given. The interviewer is basically in control of the situation by scheduling the interview, 
controlling its pace, and sequencing the questioning based upon pre-determined criteria. A limitation to the interview format is that beyond probing, the interviewer does not influence the responses of the participant and anonymity cannot be provided. The respondents identify themselves to the researcher and it is the researcher's responsibility to maintain that confidentiality.

The Background Questionnaire for Nurse Educators and Nurse Educator Interview Schedule (Appendix C) for this study were developed under the guidance of the researcher's dissertation committee chair. Criteria used in the development of the questionnaires came from a variety of sources: content that came from the literature, providing insight into the thoughts and concerns of nurse educators both past and present within the workforce; the researcher's own understanding of the issues obtained from 40 years as a nurse, 30 years as a nurse educator, 26 years as an associate degree coordinator, and 10 years as a Division Chair in a medium size community college; questions from other instruments used by other researchers in closely related studies, furnishing an overall rich understanding of the value of data obtained from their research; the research questions developed for this study, with the actual questions designed to elicit the information necessary to answer the research questions; and Astin's (1984) Sociopsychological Model of Career Choice and Work Behavior.

Finally, the questions reflected Lortie's $(1975$, p. 110) methodological perspectives to increase the likelihood of obtaining the true thoughts, feelings, values, or attitudes of the participants. These include personal versus impersonal referents, concrete versus abstract notions, cathected versus low-affect issues, and indirect versus direct questions. The background questionnaire provided demographic information on the participants allowing the researcher to collect basic information including age, years of work experience, 
education, and characteristics of the program in which they teach.

Expert Review of Research Instruments

Burns and Grove (2005) note that a draft of the questions used in an interview should be submitted to a group of individuals with expertise in various fields to validate if the questions are appropriate, accurate, and representative. For this study, two groups with members having expertise in nursing research as well qualitative research methods reviewed the Background Questionnaire for Nurse Educators as well as the Nurse Educator Interview Schedule. The members of the researcher's dissertation committee provided initial feedback of the content included in both instruments. Collectively, these individuals possess expertise in education, nursing, and research methods. After any revisions based on the feedback from the committee, the study will be re-submitted to the institutional review boards for human subjects research at both colleges.

Following approval from the institutional review boards at both Western Kentucky University and the University of Louisville, an additional review of the instruments for appropriateness, accuracy, and representativeness of the specifications was completed. Each of the three members of an Expert Panel were sent a letter (Appendix E) briefly explaining the study and providing opportunity for review and comments. The draft instruments and a guide containing a set of questions for their review (Appendix D) were included. Two of the three panel members were nurse educators familiar with the nursing faculty shortage (Dr. Jane Kirschling, University of Kentucky College of Nursing, and Dr. Susan Davis, Bellarmine School of Nursing). The third member of the panel, an expert in qualitative research design, is Dr. Judy Davison from Western Kentucky University. With their combined expertise, a complete assessment of the instruments for the study lended support for content validity. Any feedback from this panel was incorporated into the 
instruments prior to initiating the Pilot Study.

Pilot Study

A pilot study is a scaled-down version of the major study and can be used in qualitative as well as quantitative research designs. By conducting a pilot study major difficulties or problems can be avoided, especially with the instruments developed to collect the data (Polit \& Hungler, 1983). "In the case of interview schedules and questionnaires, it is important to know if respondents understand the questions and directions or if they find certain questions objectionable in some way" (p. 47). The pilot study can also help evaluate the methods for gathering the data. Difficulties in taping the interviews or in coding or analyzing the data could be identified (Gall et al., 1996). For this study, the community college in which the researcher was associated was used for the pilot study. Two subjects who possess the same characteristics as the population, with respect to educational attainment, years of experience, and Carnegie Classification of college size were recruited to complete the questionnaire and interview. However, the cells in which these two individuals would fit (see Table 1) will be different from that of the cell identified for the participant from this community college for the actual study. The two participants were contacted by phone enlisting their assistance. A letter describing the study was sent to the pilot subjects (Appendix D). After completing the survey and interview, the subjects were asked to provide feedback on the two research instruments and the process (Appendix D).

\section{Procedures}

A letter (Appendix E) was sent to the Nurse Administrator from each of the 16 community colleges and the four universities having associate degree nursing programs. The researcher gained access to the names of the program administrators from the 
Kentucky Board of Nursing (n.d.) website. The letter requested the nurse administrator to identify three to four faculty members who met the criteria for that site included in the letter. The nurse administrator was asked to send a copy of a letter (Appendix E) inviting faculty members who meet those criteria (up to four individuals) to participate in the study along with a cover memo encouraging them to volunteer. Faculty members who agreed to be in the study were asked to return a form with contact information so that the researcher could set up an interview date and time. If more than one subject volunteered for one of the 20 programs, the individual who best fit within the overall purposeful sample based on experience, educational level, and size of institution was chosen. Although not formal criteria for inclusion in this study, other demographic factors such as gender, ethnicity, native language, or rural/urban background were considered for these choices. In these instances, volunteers not selected were notified and thanked for their interest (see Appendix E).

If no faculty members from a specific program volunteered, the researcher contacted the nurse administrator about sending a follow-up invitation and entreaty to participate in the study. If subsequent invitations were unsuccessful, the researcher may have to change the grid (Table 1) in order to find 20 participants. After selecting one subject from each of the 20 programs, a letter (Appendix E) was sent to the representative from each college, confirming his/her inclusion in the study and indicating that an interview will be set up via phone and/or mail. The appointment for each interview was at the college where the faculty member teaches and was scheduled for approximately an hour in a location where the researcher and the subject would be able to talk uninterrupted and where responses to the interview questions can be audio taped.

Creswell (1998) provided guidelines for the interview process. These include the 
determination of what type of interview will obtain the most pertinent information to answer the research questions (for this study, semi-structured). Adequate recording devices should be used to capture all of the comments in the recording process. The location for conducting the interviews should be conducive to the interview process as well as the accurate recording of information. During the interview the researcher should adhere to the questions, complete the interview in the time specified, be courteous and respectful of the participant and the time involved, and avoid offering advice or straying from the original questions if not pertinent to the study. Burns and Grove (2005) also add that the interviewer should establish the interview environment comparable to that of a comfortable conversation. Another suggestion includes the ability to allow a pause during a moment of silence for the participant to provide additional thoughts that may take a moment to surface and explain. Note taking by the interviewer provides information on the overall context, body language, emotions, and any other pertinent circumstances that can help interpret the actual transcript.

Each participant in the study was required to sign a consent form at the time of the interview. According to Gall et al. (1996), the researcher needs to inform each individual as to what will occur during the study, what kind of information is being collected, and how the data will be used. The individual should be informed that if they are not satisfied with the explanation of the study, they could withdraw their consent without any associated penalty. If other individuals will have access to the data, the participant needs notification within the consent form. Additionally, the consent form included the approval for audio taping the interview. Since the data from the Background Questionnaire for Nurse Educators must be matched with the Nurse Educator Interview Schedule, Gall et al. (1996) noted that subjects can either make up a code or a name that can be placed on both 
instruments, or one can be assigned. For this study, a number was assigned to each participant. The authors also advised that the consent form should include the assurance of disposing of the data after the study has been completed. Burns and Grove (2005) add that the researcher should provide an opportunity for the participant to ask questions about the study and provide information on whom to contact should there be additional questions. A copy of the Nurse Educator Study Consent Form is included in Appendix F.

\section{Data Analysis}

Polit and Hungler (1983) reported that qualitative analysis begins with identifying themes and can be extremely time consuming. Data analysis "involves a validation of the understandings that the thematic exploration is providing" (p. 469). Another concern is the accuracy of the subject's thoughts and feelings. Themes can be reviewed by debriefing sessions. By using "quasi-statistics," the researcher can use an accounting system for the frequency of the themes and tallying the frequency of the themes. "The researcher tabulates the frequency with which certain themes, relations, or insights are supported by the data" (p. 470). The researcher summarizes the information that has been collected and organizes it to compare the themes identified with research questions.

To classify the information from the audiotapes the researcher can code each topic as it appears. Vierra et al (1998) explained that this saves time and can verify the topics that the researcher anticipates as well as those that are unanticipated and are identified while reviewing the data.

The researcher used several methods to assist in the analysis of the data. Transcripts from the taped interviews with each subject were transcribed, and then read a number of times to attain an understanding for coding. The background survey completed by each subject was tabulated and the information was included in the results. Each of the 
interview transcripts were coded according to the search for themes and patterns, as noted above.

To organize the data, a matrix was developed following the works of Miles and Huberman (1994) and Anfara, Brown, and Mangione (2002). Using the research questions and subject responses, categories were identified to promote a better understanding of the information. Each question yielded data related to each sub group--educational level, years of experience, and size of college. Both the Background Questionnaire for Nurse Educators and Nurse Educator Interview Schedule were mapped to the research questions. Then the matrix was organized by combining responses from subsections into larger sections. Specifically, each question from the background survey and interview was read and coded for the 19 participants. But these responses were subdivided by years experience, educational level, and size of college, so that differences in these characteristics could be identified, if any. That process was repeated for other questions under a specific research question or sub-research question. Then the information from each item of the background questionnaire or interview schedule was combined with the other items that are part of that specific research question, providing an overall synthesis of information for that particular research question (or sub-question). This process was repeated for each of the eight research questions. Using this inductive process, across progressively larger sections of the matrix, the researcher identified categories and themes from the completed data set. This examination is first conducted segment by segment; then the data are subjected to a final holistic reading, searching for larger patterns that may not be apparent at the level of cells of information.

To facilitate the above process, a system of tags is added to the transcripts. Anfara et al. (2002) recommend the use of tagging data in order to retrieve and provide an orderly 
analysis after completing all of the interviews. In this study there were three levels inherent in the tags: (a) the respondent number from one to twenty, (b) the respondent's educational level, years of experience, and school size, and (c) the item number from which the data were obtained, the Nurse Educator Interview Schedule or Background Questionnaire for Nurse Educators, in relation to a specific research question.

Trustworthiness and Validity

Errors in a qualitative study can occur with either the data collection or analysis. It is of utmost importance that the researcher ensures the rigor of each process. Rigor is defined as "a strict process of data collection and analysis as well as a term that reflects the overall quality of that process in qualitative research" (Macnee \& McCabe, 2008, p. 170). Burns and Grove (2005) add that:

rigor is associated with openness, scrupulous adherence to a philosophical perspective, thoroughness in collecting data, and consideration of all the data in the subjective theory development. Evaluation of the rigor of a qualitative study is based, in part, on the logic of the emerging theory and the clarity with which it sheds light on the studied phenomenon. (p. 55)

Trustworthiness, according to Macnee and McCabe (2008) is inherent in the honesty of data collection. Trustworthiness comes from a relationship formed with the participants. The subjects must feel comfortable to share information--their feelings, experiences and insights--without pressure from the researcher. The use of a defined protocol for data collection provides a broad framework, yet ensures a sameness of setting and method of data collection.

In a qualitative study, transferability of concepts and themes to a different group or setting enhances the meaning and accuracy of the research. "One of the methods used to ensure transferability is to describe themes that have been identified in one sample to a group of similar participants who did not contribute to the initial data collection to 
determine if the second group agrees with the themes" (Macnee \& McCabe, 2008, p. 172). Merriam (2002) noted that the "question of trustworthiness has to do with issues of internal validity, reliability, and external validity or generalizability" (p. 31). Eight strategies for promoting validity and reliability included by Merriam are: (a) triangulation, (b) member checks, (c) peer review/examination, (d) researcher's position or reflexivity, (e) adequate engagement in data collection, (f) maximum variation, (g) audit trail, and (h) rich, thick descriptions. This researcher addressed triangulation by using two different sources of data collection methods. Using a background questionnaire and semi-structured interview completed by a diverse group of nursing faculty, data was collected and analyzed. Member checks were utilized by confirming the information collected during the interview and contacting each subject to ensure the information collected was indicative of the thoughts and feelings of the participant.

Peer review/examination by the researcher's Dissertation Chair, Dr. Stephen Miller, was completed via review of data and analysis, focusing on interpretation. The researcher maintained a critical self-reflection with caution to worldview biases, as well as her relationship to the study with regard to adversely affecting the results of the study. Attention to outliers or lack of similarities to other members' responses will be crucial to finding new patterns. Looking for saturation in the data during analysis ensures that common themes have been discovered. Diversity was assured by selecting a purposeful sample that addresses the cells from Table 1, representing different levels for experience, education, and size of institution. These subjects were representative of groups of nursing faculty in associate degree programs in Kentucky. An audit trail will be provided by carefully examining the data and providing a detailed accounting of the methods and procedures, including the use of a matrix to keep track of answers to items from the survey 
and interview schedule and the different research questions. Rich, thick descriptions provided a clear description of the study results, allowing the reader to decide whether the findings are transferable to other situations.

Using multiple data collection methods (triangulation) can eliminate biases in the evaluation of the data (Burns \& Grove, 2005; Gall et al., 1996, Macnee \& McCabe, 2008; Merriam, 2002). In the current study, the researcher utilized a variety of sources related to a specific theory in the collection of data. The use of a matrix (described above) helped to identify themes and patterns from voluminous data files. For this study, the largest organizing elements of the matrix represent the research questions. Within the RQs, the Background Questionnaire for Nurse Educators and the Nurse Educator Interview Schedule comprised the substantive content markers. Within those content areas fall specific survey items and interview questions. The smallest element of the matrix is composed of the responses of the 20 associate degree nursing participants to the two instruments. The analysis integrates these 20 responses across the smallest component, then proceeds to integrate upwards to the highest element of the matrix, the research questions. Anfara et al. (2002) caution the researcher to provide enough detail of the data analysis to "allow validity judgments to be made by the reader....[T]here is one major element that is not sufficiently addressed--the public disclosure of processes" (p. 29).

\section{Ethical Considerations}

The researcher took care to ensure that all aspects of this research study met the standard for ethical research. Federal rules for studies involving human subjects were followed. The Human Subjects Review Board (HSRB) from both the University of Louisville and Western Kentucky University require completion of an on-line training course for certification in human subject research. This has been completed by both the 
researcher and dissertation chair, as required.

\section{Anonymity and Confidentiality}

The research participant has a right to anonymity and confidentiality to the extent required by law and consistent with the procedures of a given type of research. It is important that the findings do not link the information given on the questionnaire or interview back to the participant. The participant is assured of the confidentiality and anonymity of the responses in writing and verbally. In this qualitative study, the researcher maintained a coded file that links subjects to all subcategories of data. All coded information was tagged by those categorical identifiers so that no information in the transcribed files was identifiable by name. Once the study has been completed, all links between the subjects and the data collected will be protected and kept in a locked, secure file for five years (Macnee \& McCabe, 2008). Each participant was given a consent form in writing and verbally informed prior to data collection regarding all rights to confidentiality and anonymity.

\section{Summary}

This chapter identifies the methodological issues involved in the decision making issues and perceptions of career choice from 20 associate degree nursing faculty in Kentucky. The intent of the researcher is to gain an emic meaning of the phenomenon in question with the least amount of disruption of the setting within the world of the subjects.

The research questions were constructed following previous studies, gleaning information from those studies as well as the instruments used. In particular, research studies using Astin's Sociopsychological Model of Career Choice provided information assisting in the development of the Background Questionnaire for Nurse Educators and the Nurse Educator Interview Schedule. 
This qualitative study collected data utilizing two researcher developed instruments. The Background Questionnaire for Nurse Educators was designed to collect demographic information from each of the associate degree nursing faculty members. The Nurse Educator Interview Schedule was used to gather the emic perspective using a semistructured interview format.

Issues concerning the population and sample as well as the procedure for conducting the qualitative study were addressed. The establishment of validity of the instruments was addressed by an expert panel of three educators who have expertise in their individual fields of nursing education or qualitative research. A pilot study was conducted to provide the researcher with initial information on the actual completion of the questionnaire and the conduct of the interview. Ethical issues were discussed as well as the completion of requirements by human subjects committees for approval of the study. 


\section{CHAPTER IV}

\section{RESULTS}

\section{Introduction}

According to the National League for Nursing (2010), between 2008 and 2018, jobs in nursing are expected to increase by $22 \%$. The healthcare system is expected to generate 581,500 new jobs for registered nurses. Without aggressively recruiting for new students to commit to careers in nursing it is expected that the $\mathrm{RN}$ workforce will not meet those demands. Even if there were adequate amounts of funding and active recruitment to increase the numbers of students deciding on nursing as a career, the shortage of nursing faculty prohibits nursing programs from meeting their goals to graduate nurses in order to satisfy the demands of the healthcare system. Forty-eight percent of the nation's nursing faculty is over the age of 55 , and one-half of today's academic workforce is expected to retire by 2015 .

Nursing faculty members face a variety of challenges in their responsibilities to students and patients. Salaries in education have not kept up with those in clinical practice. Educational requirements for nursing faculty include advanced degrees in nursing. The very programs that educate nurses to teach have lured these individuals into programs rewarding advanced degrees and certifications as advanced practice nurses who can ply their trade independently with lucrative salaries. Thus, talented prospective faculty who could ease the nursing faculty shortage are lost to other nursing opportunities in advanced clinical roles, positions that are also in great demand in the healthcare 
system. Since salaries are not competitive, nurses who obtain advanced degrees and make the decision to teach have identified a career that requires additional exploration regarding that choice.

The purpose of the study was to provide insight into the factors that affect the career choice of associate degree nursing faculty in the Commonwealth of Kentucky. All of the nursing educators in this study met the Kentucky Board of Nursing requirement that nursing faculty have at least two years of nursing experience and all had made the career transition to associate degree nursing programs throughout the state. Despite minimal salary and unclear expectations, each participant articulated their past experiences and their passion to experience the ultimate outcome: success for the students in their charge. Despite their schedule and demands of coursework, responsibilities to their college, or family commitments, each one of the subjects shared their attitudes and insights into the role of an associate degree nurse educator.

The remainder of this chapter details the results of this study. Specific sections addressed include Procedures and Pilot, followed by The Findings, which are organized according to the Research Questions. The chapter concludes with a Summary.

Procedures and Pilot

After receiving approval from the Human Subjects Committees at both University of Louisville and Western Kentucky University, the two research instruments--the Background Questionnaire for Nurse Educators (BQ) and the Nurse Educator Interview Schedule (IS)--were examined for content and clarity by three educators, two in nursing and one in educational administration. The Expert Panel Members gave important feedback to the researcher. Each panel member completed the Expert Review of Background Questionnaire for Nurse Educators and the Expert Review of Nurse 
Educator Interview Schedule (Appendix D). Minor revisions to the Background Questionnaire for Nurse Educators entailed re-ordering the question to elicit the participant's age further into the questionnaire with the concern that there would be some sensitivity to that being the beginning question. A change in the options for Marital Status included Domestic Partner. The completed Expert Review of Nurse Educator Interview Schedule by each panel member provided a comprehensive review. Changes in the interview questions included a modification of wording for several questions. The feedback was incorporated into the two instruments, in consultation with the researcher's dissertation advisor.

A pilot study was conducted to test the procedures as well as an additional evaluation of the instruments. The participants for the pilot study were nursing faculty who met the criteria of the study but were not included in the research study itself. The participants of the pilot study, following the completion of the Background Questionnaire for Nurse Educators and the Nurse Educator Interview Schedule, were thorough in their evaluation of the process and the instruments and completed the Pilot Study Participants' Review of Background Questionnaire for Nurse Educators and the Pilot Study Participants' Review of Nurse Educator Interview Schedule (Appendix D). In addition to the completion of the instruments, the researcher reviewed the process and was alert to the overall observation of the subjects during the process. Following each pilot study interview the researcher reviewed the audio recordings for clarity and efficiency of transmission.

The research study began after the expert panel members and the pilot study subjects had provided input and acknowledgement that the instruments were appropriate for this project. Final revisions for both instruments were based on two rounds of 
feedback. Additionally, the researcher observed the pilot participants in their professional milieu in an attempt to understand the emic perspective of these educators as revisions were considered.

\section{Methodological Considerations}

The findings of the study are reported below and are based directly on the two instruments, The Background Questionnaire for Nurse Educators and the Nurse Educator Interview Schedule. The analysis and synthesis of the various types of data form the basis for the Discussion section and further conclusions in Chapter V.

The study addresses the overall central research question, "What factors influenced the career choice of nurse educators currently teaching in Kentucky associated degree nursing programs?" The framework for this study is grounded in literature pertaining to nursing educator faculty shortages, job satisfaction, and career choice. The Sociopsychological Model of Career Choice by Helen Astin (1984) provides theoretical direction to the study. The results from the questionnaire and interview schedule, as well as the researcher's observations during the interviews, constitute the data from which subsequent analyses are drawn. The seven research questions guide the organization and synthesis of the data within the theoretical framework.

\section{Instrumentation}

The Background Questionnaire for Nurse Educators served as a tool to obtain demographic data on each participant. Family Background data was included on the form. The responses from the questionnaire provided an insight into the family context, educational attainment, years of nursing experience, and the number of years in teaching. The results from the questionnaire are identified by the specific questionnaire item (BQBackground Questionnaire and IS-Interview Schedule) as mapped to the research 
questions (Appendix A).

The Nurse Educator Interview Schedule served as the tool to collect the detailed data in relation to the research questions. Prior to the interview each participant received a copy to follow along as the researcher questioned the subject. The goal of the interview was to gather insight into the experiences and feelings of associate degree educators in Kentucky. Through this rich qualitative database, the emic understanding of career choice was explored.

\section{Data Collection}

The research study began with the researcher contacting by letter all of the Nurse Administrators in the 20 associate degree programs in Kentucky (Appendix E). The letter identified the necessary descriptor (experience, education, and size of program) needed for the study participant at each location. Included was the Contact Letter to Nursing Faculty Member (Appendix E). The nurse administrator was instructed to develop a memo to encourage nursing faculty in the college, meeting the specific criteria for that program, to agree to participate.

The researcher then contacted the nurse administrator. In some cases, faculty had already returned the signed consent to participate forms and those were subsequently forwarded to the researcher. In other cases, either the nurse administrator or the faculty member contacted the researcher directly with a desire to participate. The letter to the administrator details which descriptor from the Purposeful Sampling Grid Across Three Factors was needed from that program. Immediately after receiving the letter, some nurse administrators contacted the researcher to report that there were no faculty meeting the set criteria. The researcher then sent electronically a form requesting the faculty make up according to the descriptors for the study. Once the researcher received a response, 
another letter was sent with the descriptor that would allow for faculty participation. For one college the faculty make-up did not meet the descriptor. Once participants had been identified for each program, the researcher contacted the faculty member and an appointment was made for the interview at the subject's college. If there was more than one faculty member meeting the criteria who wished to participate, the researcher randomly chose the subject to be interviewed. A letter of Notification of Nonparticipation (Appendix E) was sent to the other interested faculty members. Due to the extensive travel required to meet with faculty members from the associate degree programs throughout the Commonwealth, it was necessary to propose several dates that would fit into the schedules of both the educator and the researcher's travel.

All faculty members, with the exception of two, were interviewed at their college within their department. One faculty member was interviewed at the hospital in the nursing program's service area following a scheduled clinical, the other in a hotel room prior to a nursing conference. Each participant was given the Informed Consent to sign and the researcher paused to answer any questions if necessary. The participant then completed the Background Questionnaire for Nurse Educators and the interview process began. Once the interview began, it was the goal to avoid interruptions in an effort to assure focus on the responses. The participant received a copy of the Nurse Educator Interview Schedule to read along as the researcher asked the questions and audiorecorded the responses. Following transcription, the participants received an electronic copy of the summary to verify their responses.

Nineteen of 20 colleges participated in the study. One college was unable to participate because the qualifications of current nursing faculty did not meet the criteria for the study. The 19 subjects who agreed to participate were cooperative, thoughtful, 
passionate, and animated in their responses to each of the 43 questions on the IS.

The Findings

Using the purposeful sample matrix as detailed in Chapter III, the researcher coded each of the Background Questionnaires for Nurse Educators with an individual identification number. That number was also placed on the Nurse Educator Interview Schedule transcripts. Raw data from each of the 19 participants' responses to each of the 10 items on the Background Questionnaire for Nurse Educators was entered into a spreadsheet for purposes of organization. Thus, the Coded Master Analytical Tool (Matrix) included each subject's identification number along with the sub-categories for: Size of College ( $1=$ Small College; $2=$ Medium/Large College), years of nursing faculty experience (0-5 years--A, 6-10 years--B, and 11+ years--C), and educational attainment (divided into Less than a Master's, Working on a Master's, Master's, Working on a Doctorate, Doctorate). Demographic information from the BQ was also added: Age, Level of Education for Mother, Level of Education of Father, Marital Status, Number of Dependent Children, and Caring for one of more adult relatives. A black and white example of the Coded Master Analytical Tool is found in Appendix I.

After loading all of the background data into the spreadsheet, a total of 166 pages of raw transcripts from the Nurse Educator Interview Schedule were inserted into the spreadsheet, the Coded Master Analytical Tool, for each of the 43 questions from each of the 19 participants. In total, responses from the ten questions from the Background Questionnaire for Nurse Educators and 43 questions from the Nurse Educator Interview Survey were entered. To analyze the data, each category was filtered for each of the variables, educational attainment, years of nursing faculty experience, and size of college. Also included in the filtering process was age, years of nursing experiences, level of 
education of mother and father, number of dependent children, and responsibility for adult relatives. To analyze responses to the IS questions, transcript data were entered for each question for each participant. Thus, for each of the 43 questions, the response from each of the 19 subjects to that question was entered into the corresponding cell of the Coded Master Analytical Tool.

Each of the 19 participant's responses to the 43 questions were read, reread, and summarized for relevance to the questions, then entered into the Summarized IS Questionnaire Response Matrix by each of the three variables; educational attainment, years of nursing faculty experience, and size of college. The variables, Educational Attainment and Years of Experience, were color coded according to each individual classification. The Size of College variable was identified by color of text (black or red). One Matrix was developed for each of the 43 questions (example in Appendix I).

Using a cross-case analysis (Miles \& Huberman, 1994) and the prior categories inherent in the research questions, the researcher searched for themes that cut across the cases. A detailed comparative analysis was conducted of the data within each category and the three main subcategories: Experience (three levels), Size of College (two levels), and Educational Attainment (five levels). The Matrix provided a means of organizing large amounts of data, consistent with the a priori set of research questions.

Coincidentally, the matrix conformed to the standard of transparency of qualitative data (Anfara et al., 2002).

The process allowed the researcher to identify themes and patterns that were present in the data, specifically how these nursing faculty members viewed their world and their experiences. Therefore, the prioritization of the research questions constituted the framework for exploring the meaning of the subjects. Each subpart of the research 
question is repeated for the convenience of the reader. The actual question from where the question is derived is repeated immediately after the sub-research question: Background Questionnaire for Nurse Educators (BQ) and Nurse Educator Interview Schedule (IS).

\section{Research Question 1}

To what extent are personal and demographic factors influential:

1.a. Age?

Demographically, the sample of nursing faculty ranged in age from 28-65 and provided an excellent representative sample of faculty in all groups, educationally, as well as years of nursing faculty experience.

BQ3. Age:

Table 2

Current Age Range for Nursing Faculty Members

\begin{tabular}{lll} 
Age range & $f$ & $P$ \\
\hline $26-30$ & 2 & 10.5 \\
$31-35$ & 2 & 10.5 \\
$36-40$ & 1 & 5.2 \\
$41-45$ & 3 & 15.7 \\
$46-50$ & 2 & 10.5 \\
$51-55$ & 4 & 21.1 \\
$56-60$ & 3 & 15.8 \\
$61-65$ & 2 & 10.5
\end{tabular}

Note: Percentages do not sum to 100 due to rounding 
Table 2 breaks the age distribution into frequencies and percentages. The average age of the faculty members was 47.3 years, the youngest was 28 years, and the oldest was 65. There were four faculty members in the age range of 51-55, the in any age group. This is consistent with the average age of nursing faculty nationally (54.5 years) (NLN, 2010). There was an appropriate representation of subjects from the two classifications of college size, small and medium/large.

Educational Attainment by age. In the "Less than a Master's" and "Working on a Master's" categories combined, the three faculty were 28, 33, and 34 years of age with an average of 31.6. The mean age of the faculty in the "Master's" category was 54.7 years. The youngest subject was 45 years of age and the oldest 65 . The "Working on a Doctorate" category had five subjects with an average age of 44.8 years (youngest being 30 and oldest 58). In the "Doctorate" category there were four faculty; the youngest was 36 , the oldest, 56 , with the mean 49.5 for that group.

Years of Experience by age. In the "0-5" category there were seven faculty members with an average of 38 years and a range from 28 to 53 . In the "6-10" category, the youngest subject was 36 , the oldest, 63 with the average 50.8 . For the " $11+$ " category there were seven faculty: mean $=54.3$, and range from 43 to 65 years.

Size of College by age. In the "Small" college category there were seven faculty with the youngest being 28 and the oldest 63 , the average was 47.1 years. In the "Medium/Large" category the average was 47.5 with a range of 33 to 65 .

1.b. BQ4: Marital status?

BQ4. Marital status:

Fifteen faculty members were married (one widowed), two were single, and two 
had domestic partners. There did not seem to be any pattern for marital status across the three primary subcategories as noted below.

All three of the participants in the "Less than a Master's and "Working on a Master's" categories are married. In the "Master's category six of the seven were married, one was single. In the "Working on a Doctorate" category all four were married. Of the four in the "Doctorate" category, one was married, one was single, and two had domestic partners.

For "0-5" years of experience, six were married and one had a domestic partner. In the " $6-10 "$ category one had a domestic partner and four were married. In the " $11+$ " category there was two single and five married. There were six married faculty and one single subject in the "Small" college category. In the "Medium/Large" category of the 12 subjects there were nine married, 2 had domestic partners and one was single.

1.c. Current family responsibilities?

BQ5. Current family responsibilities:

Nine of the subjects had dependent children. Two of the faculty had adult children. There were eight with no children but one of those cared for one or more adult relatives. One faculty member had four children which was the most for a single subject. Across the three subcategories below, there was no discernable pattern for family responsibilities across educational level or size of college. For years of experience, those with no children were concentrated in the "6-10" years and " $11+$ " categories.

Educational Attainment by family responsibilities. Of the three faculty making up the "Less than a Master's" and "Working on a Master's" categories, one had no children, one had one, and one had two. In the "Master's" category, the five subjects had a total of 10 children. Two faculty had no children, with one faculty member having four adult 
children. In the "Working on a Doctorate" category, three of the five faculty had a total of five children. Two of the faculty had two children each. Of the four faculty in the "Doctorate" category one has an adult child and another has two dependent children. One member of this category had no children but is caring for one or more adult relatives.

Years of Experience by family responsibilities. There were a total of 12 children from six of seven faculty in the " $0-5$ " years of nursing faculty experience category. One participant had three children. In the "6-10" category there was one faculty member with one child. The other four had none. In the " $11+$ " category there were nine children. One faculty member had four children, one had two, two had one child each and three had none. One member was caring for one or more adult relatives.

Size of College by family responsibilities. The faculty members teaching in the "Small" college category had a total of seven children. One had four and one had no children but was caring for one or more adult relatives. Faculty in the "Medium/Large" category had 14 children with one subject having three children while eight had none.

\section{1.d. Years of nursing experience?}

BQ6. Years of nursing experience:

The average number of years of nursing experience was 23.5. One faculty member had been in nursing seven years and the person with the most years was 40 . Sixty-eight percent of the subjects had been in nursing for over 20 years. Only 3 or $16 \%$ had been in nursing less than 10 years.

Table 3 below, gives the frequency and percentages across the experience levels. The greatest majority of subjects ( 8 ) had been in nursing from 21-30 years. Thirteen of the faculty $(68 \%)$ have had more than 20 years of nursing experience. This is an experienced group who has clinical 
Table 3

Years of Nursing Experience

\begin{tabular}{lll}
\hline Experience range & $n$ & $P$ \\
\hline$<10$ & 3 & 15.7 \\
$10-20$ & 3 & 15.7 \\
$21-30$ & 8 & 42.1 \\
$31-40$ & 5 & 26.3 \\
\hline
\end{tabular}

Note: Percentages do not sum to 100 due to rounding

Educational Attainment by nursing experience. The three faculty in the "Less than a Master's" and "Working on a Master's" category have had 28 years of nursing experience for an average of 9.3 years; the most is 12 and the least is seven. In the "Master's" category the average participant has 27.6 years with a range from 19 to 40 . The average number of years in the "Less than a Doctorate" category is 22 with one member having practiced as a nurse for eight years and the highest years of practice 37 . In the "Doctorate" category the least number of years of nursing practice by a participant is 18 and the most 38 with an average of 27.3 years.

Years of Experience by nursing experience. The faculty in the " $0-5$ " years of nursing faculty experience category had an average of 16.1 years of nursing experience and ranged from seven to 31 . For "6-10" years of nursing faculty experience, the least amount of nursing experience for one participant was 18 and the most was 37 with a mean of 26.6. In the " $11+$ " category the average number of years of nursing practice was 25.6; the range for practice was 19 to 32 . 
Size of College by nursing experience. The mean years of nursing practice of the participants in the "Small" college size category is 20.2. The lowest number of years of practice is seven and the highest is 31 . In the "Medium/Large" category the least number of years of nursing practice is nine and the most is 40 with an average number of 24.8 years of nursing practice.

1.e. Years of nursing faculty experience?

BQ7. Years of nursing faculty experience:

The average number of years of nursing faculty experience was 10.3. The least number of years as a full time faculty member was two months (but had been employed three years in a part-time role) and the longest employed faculty member in nursing education was 32 years. Thirty-two percent of the participants had been in nursing education for less than five years. Eight subjects (42\%) had been working as nursing faculty from 10-20 years. There were two who had worked in nursing education for 28 and 32 years.

Educational Attainment by nursing faculty experience. In the "Less than a Master's" and "Working on a Master's" categories the number of years of nursing faculty experience is an average of 1.3 years. There are two faculty in their first year, the other faculty member has worked two years. In the "Master's category" the average number of years is 14 . There is a faculty member who had been employed for just two months fulltime (three years part-time) and one who had been a nursing faculty member 32 years. Fourteen years is the average number of years teaching experience for this "Master's" category. The "Working on a Doctorate" level had five faculty with the member having the least number years of teaching at four while the most was 13 . The average number of years of teaching for this group is 6.8. The "Doctorate" category had an average number 
of years of faculty experience at 14.75 with the lowest number of years being 3 and the highest 28. As would be expected, for both education degrees and experience subcategories the trend was for fewer years as nursing faculty across these two dimensions in the lower levels. There was essentially no difference for faculty experience by size of program.

Years of Experience by subcategories. In the "0-5" years of experience group there were seven participants with an average of 2.3 years of teaching experience. The faculty member with the least amount of time was two months of full time teaching ( 3 years part-time) and the subject with the most number of years in that category was five. In the " $6-10$ " years of nursing faculty experience the average number of years is 8.4 . The least amount was six and the most 10 . Three faculty had 10 years of experience and two had six. In the "11+" group there were seven faculty with an average number of years of teaching experience at 19.6. The least amount of experience was 11 and the most was 32 . Size of College by nursing faculty experience. The faculty making up the "Small" college category had an average number of nursing faculty experience of 9.9 . The least number of years was one; the most was 18 . The "Medium/Large" group had an average number of years of nursing faculty experience of 10.5 with the least amount of experience two months and the most 32 .

\section{1.f. Family educational attainment?}

BQ8. Mother's educational attainment:

Eighty four percent (16) of the mothers had less than a college degree while three of the mothers had a college or professional degree.

Educational Attainment by subcategories. In the "Less than a Master's" and "Working on a Master's" category one faculty member's mother had a high school 
education and two had mothers with some college. In the "Master's" category, respondents recorded that one mother had less than high school, three finished high school, one had some college, one had completed college, and one had a professional or advanced degree. In the "Working on a Doctorate" category, one of the mothers had less than a high school education and the other four had a high school education. The mothers of the members in the "Doctorate" category had two with less than high school, one some college, and one with a college degree. For this small sample, there appeared to be no association between working on or having an advanced (doctorate) degree and mother's educational level.

Years of Experience by mother's educational level. For the mothers of the members in the " $0-5$ " category, two had less than high school, three had completed high school and two had some college. In the "6-10" category, one mother had less than a high school diploma, three completed high school, and one had some college. The " $11+$ " group had mothers with the most formal education. One mother had less than a high school diploma, two had high school, one had some college, two had college degrees, and one had a professional or advanced degree.

Size of College by mother's educational attainment. For the mothers of the members in the "Small" college category, one mother had less than high school, three had high school, two had some college, and one had a college degree. Members' mothers from the "Medium/Large" category reported that three had less than a high school diploma, five had a high school diploma, two had some college, one had a college degree, and one had a professional or advanced degree.

BQ9. Father's educational attainment:

Fathers' educational attainment revealed $79 \%(15)$ had less than a college degree 
and four had a college or professional degree.

Educational Attainment by subcategories. In the category of "Less than a Master's" and "Working on a Master's," fathers of respondents reported that one completed high school and two had some college. In the "Master's" group, fathers were reported as one with less than a high school diploma, five with a high school diploma, and one with a professional or advanced degree. One father had less than a high school diploma in the "Working on a Doctorate" group, two completed high school, one had a college degree, and one had a professional or advanced degree. Two fathers in the "Doctorate" group had less than a high school diploma, one had some college, and one had a professional or advanced degree. As with the mothers' educational attainment, fathers of these 19 nursing faculty were scattered across the five levels of degree completion in no discernable pattern.

Years of Experience by with fathers' educational attainment. In the " $0-5$ " group two fathers had less than a high school diploma, three had a high school diploma, and two had some college. In the "6-10" group one father had less than a high school diploma, three completed high school, and one had a college degree. One father in the " $11+$ "group had less than a high school diploma, two completed high school, one had some college, and three had professional or advanced degrees. The researcher found no pattern in degrees completed for years of nursing faculty experience.

Size of College by fathers' educational attainment. The fathers of members who taught at "Small" classification colleges reported that one father had less than a high school diploma, four had high school diplomas, and two had some college. In the "Medium/Large" group three fathers had less than a high school diploma, four completed high school, one had a college degree, and three had professional or advanced degrees. 
Again, there was no pattern for fathers' educational attainment and size of program.

1.g. Educational attainment?

BQ10. My educational attainment:

Educational Attainment by subcategories. In the "Less than a Master's and

"Working on a Master's" category there were three faculty with a Bachelor's degree. In the "Master's Degree" there were seven with a Master's degree. In the "Working on a Doctorate" category there were five working on a Doctorate. In the "Doctorate" group there were four with a Doctorate.

Years of Experience by nursing faculty educational attainment. In the years of experience "0-5" group, there were two nursing faculty with a BSN, one working on a Master's, one with a Master's, two working on a Doctorate and one with a Doctorate. In the "6-10" group there were two with a Master's, two working on a Doctorate, and one with a Doctorate. In the " $11+$ " group there were four Master's faculty, one working on a Doctorate, and two with Doctorates. As would be expected, the two lowest levels of degree attainment were found in the " $0-5$ " level of experience. In all three levels, there were faculty with or working on a doctorate.

Size of College by nursing faculty educational attainment. In the group "Small" college there was one faculty member working on a Master's, three with Master's, two working on a Doctorate, and one with a Doctorate. In the "Medium/Large" college category one faculty member had less than a Master's, one was working on a Master's, four had Master's, three were working on a Doctorate, and three had Doctorates. Again, no pattern existed for size of college in this sample for nursing faculty educational attainment.

1.h. Influential person or event? 
IS1. Tell me when and why you decided on nursing as a profession?

For this subquestion, responses came from the Nurse Educator Interview Schedule rather than the Background Questionnaire for Nurse Educators. The four IS questions included in the first Research Question 1 focused on the "personal" reasons for becoming a nurse educator rather than demographic sections from the Background Questionnaire for Nursing Educators.

Faculty chose nursing as a profession for a variety of reasons: many had relatives that were healthcare providers, some for situational reasons, some wanted to care for people, and some had limited occupational choices in their early years and nursing was acceptable. In transitioning from a clinical nurse to nurse educator, the change was as a result of a particular situation within the clinical environment, the physically demanding responsibilities of the work environment, or the desire to pursue other endeavors.

For all of the specific questions from the Nurse Educator Interview Schedule, the decision whether to disaggregate data by the three primary subcategories was based on the degree of differentiation of responses across the 19 respondents. If answers reflected strong commonality across the five levels of educational attainment, three levels of nursing faculty experience, or two levels of size of college, then no further analysis (beyond that given in initial overall summary) is given.

Seven participants (37\%) responded that they went into the nursing profession after a relative had been either a nurse or another healthcare professional. Four of the 19 subjects responded that they had a relative who was ill or who had died and from that experience they decided on nursing. Sixteen percent (3) of the respondents stated that they went into nursing to care for people. "I always felt like I was a nurturer. I think that early on I wanted to be a nurse. I was a candy striper and all that; then pursued things like 
a nurse aid and worked in Central Supply one summer just to get my foot in the door. Just always follow my dream." Another respondent told of her family as a group of educators. Their comments drove her from education initially. She also thought about law school but women were entering the more traditional teaching and nursing roles in the late $1960 \mathrm{~s}$ and early 1970s. She decided on nursing, "I thought it was an opportunity. I knew it was going to be hard work, but it looked like it would be fun. It would be an opportunity to challenge myself, and it did, nursing is a challenge to me." Another faculty member had a similar experience in the 1960 s as she graduated from high school and wanted to go to medical school. At that time she says, "they were only admitting 4.0 [GPA] males. Getting a job in the sixties was quite the battle. I got interested in medical programs....Then I pursued a nursing degree and finished." Another respondent had a father who owned a nursing home. "My Dad owned a nursing home and we had fun relationships with patients and stuff. My Dad and brother told me I needed to go into nursing and that is the only reason I did it."

Educational Attainment by when and why nursing. There were two faculty members in the "BSN" category and one in the "Working on a Master's" category. For data analysis, the one subject in the "Working on a Master's" category will be considered as part of the BSN group. This faculty member has a BSN and has begun work on her Master's. Two of the group had relatives who are nurses, the other stated that she liked caring for people.

Years of Experience by when and why nursing. Three of the seven members in the category of " $0-5$ years" of experience cited family members in nursing as the reason for becoming a nurse. Three of the seven wanted to care for people and one nurse had a family member who was sick and died; she witnessed good and bad nursing care. She 
stated that she wanted to go into nursing to make a difference in patient care. Of the five members in the category of 6-10 years of experience, one had a relative in nursing, one had a sick relative and the situation led her to nursing, one had a strong science background that led her to nursing, and two had experiences that led them to nursing as an alternative career.

Of the seven nurse faculty members in the $11+$ years of experience category, five had relatives who were in the health field, one always wanted to be a nurse, and one settled for nursing because of not getting into medical school.

Size of College by when and why nursing. Three of the seven faculty in the small college category had relatives in the healthcare field, four had situations where an illness of a relative made the difference, and one had an unusual experience where as a high school senior she was offered a job as a secretary. Since she typed so poorly she announced that she wanted to go to college and become a nurse. The "Medium/Large" group had similar responses but also "caring for people" was included. Two always wanted to be a nurse.

IS2. What influenced your decision to become a faculty member?

Nurses who made the change to teaching were supported by program faculty and administrators. Many of the subjects spoke of their "love for teaching" and their desire to work with students as an important part of their role in academe.

Responses to this question yielded a variety of comments, some of which included: the subject had a love of teaching and nursing ( 2 respondents), and had come from a family of educators and were familiar with some of the responsibilities ( 2 respondents). Two faculty had a teaching role in the hospital setting and made the transition to academe. One noted: 
If you would have told me that I would be teaching I would have laughed at you and it would have been totally the furthest thing from my mind. When I was working as a staff nurse I got involved with the hospital education committee. We were doing a lot of things with nurses and teaching patients. I really enjoyed that. I did go back and get my Bachelor's and I did not consider teaching until I saw an advertisement for clinical instructors at a local community college.

Further, she related that she liked the clinical role and really enjoyed the opportunity.

Three faculty told of their need to change their role due to the increased responsibilities

of working in the clinical setting. One said:

I think my biggest decision was my last job; I was rotating 12 hour shifts in NICU three weeks days and three weeks nights. I decided I needed a change. Since I had finished my Master's I felt that that was the direction I needed to go. I did not have a life. I was single and I thought life was passing me by.

One faculty member related having one of many bad days in ICU (Intensive Care Unit) at the local hospital:

The day from hell in ICU, I still cringe at the thought of that day. I had a young person code on me, and another one code. Everything was going wrong for me that day. When I went home I went through the door and prayed and asked God for something else for me.

She relates that she received a phone call a short time later informing her of a job opening at the college. She felt like it was a message to her to make the change.

Another nurse related her love for humor and love of learning and the challenge to present content in such a way that students can grasp with less difficulty. She stated that she has planned three careers in her life: nursing at the bedside, teaching, and a third undecided career. When asked what areas for the third career she seriously replied, "stand up comedy."

Three faculty had relocated to the area and instead of applying to work at the local hospital they came to work at the college. Two had clinical positions on a part-time basis and had the opportunity to go full-time and take a didactic and clinical position. 
Educational Attainment by what influenced decision. Of the three participants who made up the categories, "Less than a Master's and "Working on a Master's," there are differing reasons for their decision to teach. One had a love of learning and teaching, one had been a clinical preceptor and was able to take a part-time position in clinical, and one had not been interested in teaching but was offered and accepted a part-time clinical position before moving to a full-time position.

One third of the participants in the "Master's" category identified a need for a change, two had relatives in education, one had recently relocated to the area, and the last one had an offer to teach and "decided to give it a try."

The "Working on a Doctorate" category had two faculty who needed a change and three had teaching roles in the hospital setting and decided to make the move when the opportunity was available. In the "Doctorate" category, one relocated to the area and took a teaching job, two stated they thought they could make a difference in influencing students, and one originally graduated from an ADN program and saw the need to obtain additional education in an attempt to position her for a better job in nursing.

Years of Experience by what influenced decision. In the " $0-5$ " category one faculty member wanted a change from the hospital setting to teaching, two had a love for teaching and learning, two had a part-time clinical role and made the change to full-time, and one had a teaching role in the hospital setting and wanted to move into education.

It was similar in the "6-10" category, two wanted a change, one came from an educational family background, one wanted to influence the "product" from nursing education, and one was interested in the teaching role after being offered the position to teach.

The most experienced educators in the " $11+$ " category had three who relocated to 
the area and accepted a teaching position, two wanted a change, one was interested in teaching more for self, and one came from an educational family background.

Size of College by what influenced decision. In the "small college" category, there were similar responses to deciding on a position as faculty. Two subjects were in nursing education in the healthcare setting and moved to academe. Another group had two subjects who had relocated to the area and applied to the college to teach. The main reason that participants from small colleges became nursing faculty centered around the love of teaching and learning and for some, the previous position in healthcare provided an opportunity for them to teach staff and patients as they felt they could make that transition to teaching in academe. In contrast, large college subjects were more likely to relocate to the area where the college was located and take a teaching position.

IS3. Was there a key person/situation that supported your decision to teach? In what way?

Six respondents related support from other faculty who were currently teaching in the nursing program. Several subjects responded they had been taught by faculty members and had the opportunity to teach with them. "The division chair of that program at the time was very much a role model. I reached a point in my clinical career where I was looking for something different. She was a big influence in my decision to teach." For another faculty member the coordinator of the program was a personal friend and offered her a position at the community college in her hometown.

Educational Attainment by key person/situation. Respondents in the "Less than a Master's" and "Working on a Master's" categories cited the current faculty who were supportive of them. For one subject a relative was a faculty member and mentored her with her first clinical group. The third subject mentioned her Mother as being supportive 
of her transitioning from clinical to teaching.

In the "Master's" category five of the seven respondents spoke of the support they received from present faculty or administrators. Two were approached and supported by the Program Coordinator to become part of the faculty. Two were impressed by faculty who taught them in the program and their decision to teach was as a result of wanting to work alongside them. One was introduced to the college by her neighbor who knew that she was looking for a job. "I was moping around the house, my next door neighbor was a nurse and she introduced me to the college and I have been there ever since." The last participant noted that, "I watched my Mother bring home homework and never thought I would do that." From the "Working on Doctorate" category, two faculty responded that they were supported by current faculty teaching. The faculty member taught alongside her and was instrumental in getting her hired. At the time she had a BSN and agreed to work on her Master's while continuing to teach. "They were willing to take a chance on me, I was very grateful to them." The last subject related that she relocated to the area and began as a clinical instructor. "I loved being in a clinical position with the students." Two respondents in the "Doctorate" category responded that they could not add anything further to the information. Two related support by faculty or administration in the decision to teach. One noted that the Division Chair was a role model. The other subject stated that the decision to take a full time position was made after the experience of being a lab instructor and received the support of the other faculty within the program.

Years of Experience by key person/situation. Of the seven subjects who represent the category " $0-5$ " years of nursing faculty experience six responded to the question. Five of the six mentioned the support of the faculty or program administrators who were teaching in the courses. One faculty member related that the program coordinator was a 
friend of hers and encouraged her to return to school to obtain her Master's even though she was still working in the clinical setting. She continued to encourage her until she completed her education and offered her a part-time clinical position which led to full time. "She plugged me into lecturing and mentored me. She kept saying you can do this." In the "6-10" category four of the five respondents added that the faculty or the Division Chair had been supportive. Another subject detailed the role her neighbor had in assisting her to find a position at the community college. The fourth respondent added that she took a position as a clinical instructor and liked it so much she wanted to continue. In the " $11+$ " category responses resembled those of faculty with less teaching experience. Four of the seven discussed the support they received by the faculty. One faculty member had been a student to the very faculty with whom she was currently working. They were influential when she was a student in the program and she is quite proud of being part of the faculty and working with them. One faculty needed a change, while one subject saw her Mother (a teacher) bringing home work and spending a great deal of time completing it.

Size of College by key person/situation. Three faculty in the "small" college category cited their fellow faculty or program coordinator as being instrumental in their decision to teach. Two faculty related that their neighbor or mother supported them in their decision and two stated they had answered the question in the previous items. In the "large" category there are eight faculty who related the support of the faculty or program coordinator with whom they were working, one related it was time for a change, one took a position as a clinical instructor and loved it, one described her mother's experiences as a teacher and the demands necessary, and one had nothing additional to add.

1.i. College classification-Small or Medium/Large? 
Selected by researcher from institutional criteria.

Using the Carnegie Foundation Classification of Institutions of Higher Education (n.d.) and the latest designation of size of college, the 20 public colleges with associate degree nursing programs were categorized as small, medium, and large. The medium and large colleges were merged into the medium/large category. There were eight schools classified as small and 12 medium/large schools. One college classified as large was not included in the study because the faculty currently teaching in the program did not meet the descriptor designated for the study.

1.j. Other?

IS4. Anything else you would like to add about the personal or background factors that influenced your decision to teach?

This question was a continuation of additional thoughts identified by some of the faculty members in the first three questions. Several faculty (3) responded that they had a love for teaching. "I love teaching. I love teaching. I teach fundamentals of nursing (the first nursing course) and have for 10 years. I haven't regretted one moment even though it seems very time consuming."

Four of the faculty did not answer the question having no additional comments to make. One respondent declared that she saw her role with students as "that cheerleader in your corner. There is always one faculty member that tells you, 'yes you can do this'. I thought I could be that person."

Educational Attainment by other. Of the three faculty in the categories, "Less than a Master's" and "Working on a Master's," one mentioned that she wanted to be the cheerleader as mentioned above, one had a stepmother who is a doctor who she feels influenced her healthcare choice. The third faculty member in the category responded 
that "nothing came to mind" to add to the question. In the "Master's" group one subject felt that she needed a change to have a more normal schedule than what she had in the clinical setting, two received support from faculty or the program coordinator, one faculty member had a relative that had an advanced degree and read to her when she was a small child. The relative told her she would love teaching. Another faculty member extols her love for teaching, and another added that she should have always been a teacher. "I originally wanted to teach history, but there were no teaching jobs in Kentucky. When I get through with this job I'm going to take my history classes and teach."

In the "Working on a Doctorate" group three faculty had similar themes in that they loved to teach. One stated she loved putting together a lecture, one enjoyed the student interaction, and another enjoyed teaching and being with students. One faculty member had support of aunts and uncles. She had listened to them relate their "bad days." "I did not want any part of that then. Now here I am; I have come full circle." Of the four faculty in the "Doctorate" group the responses to the question yielded three varied responses. One subject answered that "teaching keeps me on the cutting edge of theoretical knowledge. I am continually refining my knowledge." One faculty member stated, "I almost quit the first year. I couldn't get from staff nurse to the teaching mode." The third faculty member never thought she would "end up in nursing education." Her experience in teaching new nurses in the clinical setting led to her accepting a teaching position.

Years of Experience by other. In the " $0-5$ " category one wanted to be supportive of students, one had a supportive relative, another wanted a change, and one enjoyed teaching. In the "6-10" years of nursing experience category, three faculty answered the question similar in the content of teaching. One in responding to the question, placed her 
hand over her heart, smiled and remarked that she "really enjoyed teaching and being with students." One faculty member related the strong push coming from the program coordinator for her to get her Master's to qualify her to teach. In the "11+" group, all who responded mentioned teaching either that they loved it or they had difficulty in the transition from the clinical setting to academe.

Size of College by other. The faculty representing the "Small" college category described their desire to support students and that they received support from faculty to pursue their interest in nursing. In the "Medium/Large" group the faculty responses centered around the desire to make the change from clinical nursing to teaching, as well as the support from family and fellow faculty members in making the decision.

BQ1. Gender:

Gender was not a sub-research question but that information is included here under RQ1.j, Other. There were 18 females and one male who participated in the study. For the three primary subcategories for this study, that one male nursing faculty was located, respectively in the "Doctorate," for educational attainment; in the "6-10" years of nursing faculty experience category; and the "Medium/Large" category for college size.

BQ2. College where employed:

Sixteen of the respondents were from a community college within the Kentucky Community and Technical College System. The other three subjects were from 4-year colleges in the state with associate degree nursing programs. With respect to educational attainment, one of these three was in the "Master's" category, one in the "Working on a Doctorate" category, and one had a Doctorate. Regarding years of experience, there was one of the 4-year college faculty members in each level: $0-5,6-10$, and $11+$. For size of college, one of the 4-year institution faculty was at a "Small" college, two worked in the 
"Medium/Large" category.

Research Question 1 Summarized

Research question one and its subparts (a. - j.) detailed the responses to demographic characteristics as well as four interview schedule questions. The responses were organized to capture not only individual responses but the patterns of the overall group plus the three primary categories-- educational attainment, years of teaching experience, and size of college where the nursing program is located. The demographic responses provide insight to the personal characteristics of the research subjects. Demographically, the group as a whole has a wide variance in the ages of the subjects. The youngest was 28 and the oldest was 63 , a 35 year difference and a two to three generational differences. The same is true for the experience in nursing. The subject with the least amount of nursing experience had seven years. The most experienced faculty person had 40 years of nursing experience. Not all of the participants with the most education were the oldest overall. One faculty person working on a Doctorate was only thirty years of age. Another faculty member with a Doctorate was only 36 years of age and had been teaching nursing for ten years. However, $56 \%$ of the group of educators was over the age of 46 . The educational attainment of the subjects' parents provided some interesting insights. Overall the fathers had a higher educational level than the mothers. More fathers had professional or advanced degrees than the mothers. However, for both mothers and fathers, several (16) had no education beyond high school or only some college. The overall educational level of the faculty in the " $11+$ " years of teaching experience category was higher than the other two categories (0-5 and 6-10). One of the nursing faculty members in that group recorded professional or advanced degrees for both parents. 
There are four interview questions as part of Research Question 1. Responses from the subjects to the first four interview questions identified seven major themes. First, many of the subjects came into nursing from a family member who was in healthcare and who modeled the behavior and supported their decision. Second, some made the decision to go into nursing based upon a situation such as the illness of a relative. Third, some nurse educators made the decision to study nursing because they wanted to care for people, to nurture others. Fourth, others verbalized their decision to enter nursing because of the traditional roles in the 1960 s for women and the limited choices for occupations. Fifth, regarding the choice to change careers to nursing education from the clinical setting, there was calculated concern about a particular situation related to the clinical environment of nursing, such as the realization after several years in nursing that the subject could not physically handle the responsibilities of working in the clinical environment, or the desire to "do something else." Sixth, several respondents noted that the primary influence on the decision to change careers was the support and encouragement of faculty and administrators early in the process. Closely related to this, several subjects saw their teachers as their role models and were excited about the opportunity to come back and teach with them. Finally, there was substantial support for the notion of "Love of teaching" and working with/influencing student nurses.

\section{Research Question 2}

In what ways do educational degree requirements for faculty positions:

2.a. Influence decisions to become a faculty member?

Ten of the 19 faculty graduated from associate degree programs. All returned to school to complete a variety of levels of nursing: BSN, MSN, DNP or PhD. Six of the 19 
were pursuing additional educational degrees. Eight faculty had completed an MSN prior to applying to an ADN program for a teaching position. None of the faculty responded that obtaining a higher degree was the reason for making the decision to teach.

IS5. What was your original educational level for licensure? Did this influence in any way your eventual decision to teach? How and why?

Ten nursing faculty (53\%) originally obtained an associate degree in nursing as their original educational level for licensure. Six (32\%) completed a Bachelor's in Nursing program as their original licensure, and three women completed a Diploma nursing program leading to $\mathrm{RN}$ licensure. Several faculty cited the close proximity of the schools of nursing in their area. Some had relatives who had graduated from the same program. One individual reported that she chose the diploma program for the preparation of a superior nurse with excellent nursing skills.

Educational Attainment by original educational level. In the "Less than a Master's" and "Working on a Master's" categories, all three of the subjects completed an associate degree program as the initial nursing licensure. For the "Master's" group four of the nurses completed an associate degree initially while three completed the BSN as their initial educational level for nursing licensure. In the group "Working on a Doctorate" one completed the ADN, two completed the BSN, and two completed a diploma program which leads to registered nursing licensure as well. The faculty making up the "Doctorate" group had two subjects complete the ADN program, one the BSN, and one a diploma program.

Years of Experience by original educational level. In the " $0-5$ " years of teaching experience three faculty attained the ADN initially, two completed the BSN, and two were diploma graduates. Four subjects in the "6-10" category completed the ADN, and 
one completed a diploma program. Three of the participants graduated from the ADN program in the " $11+$ " group and four completed a BSN initially.

Size of College by original educational level. No differences in comparison beyond what was previously discussed.

IS6. What was your original educational level when you started teaching? Did this enter into your decision to teach? How and why?

Fifty-eight percent (11) had a BSN when they accepted a teaching position. The remaining eight had Master's degrees. One faculty member did not obtain a BSN for teaching but chose to complete the degree for additional leadership roles within the clinical setting. Only one faculty member related that obtaining a BSN, then an MSN, was an influence on their decision to teach. Another subject was in the military and completed a Master's degree but it was not in nursing. She returned to college to complete the MSN. Another faculty member related her job in the emergency room as an ADN then returned to school to obtain a BSN and MSN. After completion of the Master's she stated that her thoughts were, "I've done nothing with my education." She then sought out teaching positions. None of the faculty in the study had a Doctorate when they first began teaching. Four of the group currently have Doctorates and five are in the process of completing requirements for the degree.

Educational Attainment by educational level at career transition. In the categories "Less than a Master's" and "Working on a Master's" all three subjects started teaching with a BSN. In the "Master's" group, five had a BSN and two had an MSN. The "Working on a Doctorate" category, five had an MSN and one had a BSN as initial employment in teaching. In the "Doctorate" group two began teaching with a BSN and two had an MSN. 
Years of Experience by educational level at career transition. No differences

beyond what was previously discussed.

Size of College by educational level at career transition. No differences beyond what was previously discussed.

b. Influence career trajectory (tenure and promotion) and retention?

IS7. What are your college's requirements for terminal degrees for promotion and/or tenure? To what extent did this influence your decision to teach?

Of the 19 subjects, two related that the BSN was the requirement at the college, 12 said that the MSN was the required terminal degree, two a doctoral degree, and three did not know. Several members of the group discussed the tenure phase-out for the community college system. Two faculty stated that they were originally on a tenure track position, but according to the subjects, they had been removed from the tenure track. For the 4-year colleges in the study, all three identified the doctoral degree as the terminal degree. One 4-year college delineated that with an approved re-structuring plan, their program will become part of the school of nursing. No one indicated that this had been an influence on their decision to become a faculty member.

Educational Attainment by required terminal degree. In the "Less than a Master's" group and "Working on a Masters" two stated that the MSN was the terminal degree and one reported that the BSN was the terminal degree for her college. All six of the "Master's" group reported that the MSN was the terminal degree. One faculty member informed the researcher about the plan for the associate degree to be integrated into the college of nursing at her university and that the $\mathrm{PhD}$ will be the terminal degree. In the "Working on a Doctorate" group three reported that the MSN was the terminal degree. One subject was not sure what the terminal degree was at her college. The 
"Doctorate" group reported that the BSN is a terminal degree at one college, the MSN at two other colleges, and one college requires a $\mathrm{PhD}$.

Years of Experience by required terminal degree. No differences beyond what was previously discussed.

Size of College by required terminal degree. No differences beyond what was previously discussed.

Research Question 2 Summarized

The subjects in this study took a variety of paths to come to initial licensure as an RN. Over half of the participants completed the ADN program initially. They returned to college and completed BSN and MSN degrees as well as, for a few, the Doctorate. The group is an educationally mobile group moving from one level to another for a variety of reasons, not just to teach, but prior to that to secure additional clinical jobs with more responsibility within the healthcare institutions.

Eleven of the 19 faculty in the study had completed a BSN to begin teaching, eight had completed the MSN degree before making the transition. The terminal degree for most of the faculty teaching in the community college is a Master's. Two of the faculty from community college programs related that a BSN was the terminal degree. In the 4-year colleges, representatives in the study reported that the Doctorate was the terminal degree. None of the study participants reported that obtaining a higher degree for teaching was influential in their decision to teach, but two noted that they had been removed from tenured lines because of a change of protocol by the Presidents (a faculty member's opinion) in awarding tenure.

\section{Research Question 3}

To what extent is the work environment in academia (compared to clinical 
nursing) influential:

\section{3.a. Flexibility?}

Faculty responded that flexibility in the college setting was a key element in their decision to move from clinical to academe. Family responsibilities, the desire to impact a larger number of students, and the success of the faculty member within the teaching role were enhanced by the support of other faculty which eased the transition from clinical to academe. A number of faculty favorably rated the college environment over the clinical setting maintaining that there was more collegiality within the college settings. Likewise, $63 \%$ of the participants rated the organizational climate of the college as a benefit to teaching. Faculty also stated that there was significant prestige in working as a faculty member within the college.

IS8. Can you compare the flexibility of your teaching position within the college setting and your previous clinical position? Do you consider this a benefit or burden of teaching? Why?

Overwhelmingly the discussion of flexibility in teaching brought out many thoughts and feelings. All subjects wholeheartedly agreed that nursing education allowed for flexibility and it provided an opportunity for family life. Comparisons to the rigidity and the structure necessary for a successful patient care unit and the increased burden of work that shifts to the nurse when an employee on the unit is absent made for animated conversations and the opportunity to understand their feelings in relation to work load.

Educational Attainment by flexibility. The three faculty making up the "Less than a Master's" and "Working on a Master's" each had one year or less of teaching experience so it seemed easy for them to compare teaching to the clinical setting. All three were team teaching with one or more experienced faculty members. The "sharing of the load" was important to them in this early period of teaching. One faculty member 
added that the small numbers of students made it helpful for her to work with them. The schedule of teaching was flexible yet it provided an opportunity to plan ahead and have a "normal" day. Somewhat of a negative aspect of teaching was the time spent after the work day. "When I left the hospital I left the responsibility behind. Now that I am teaching I take homework home and I have given the students my cell phone number because I feel the need to be available." Another subject said, "Both areas are affected by the absence of someone and the others have to take an extra load. If you are teaching a class and cannot be there, someone will have to take care of your class or clinical group." Common to the first group, the "Master's" group had similar comments in relation to the question. "It is very much a benefit. In the clinical setting it does not make any difference how long you have worked there, you still are working every other weekend and holidays. Teaching allows for a family life and it takes the burden off of my husband." Another nurse educator stated, "clinical and floor work is extremely unpredictable." On the downside, one faculty member stated,"I am putting in a lot of hours up front this first year. I see it as a gift to the college." Another stated that, "I take home 1-2 bags of books every night. Teaching and bedside nursing are two different facets of nursing. It is a benefit, but I think at times it is harder than the day to day job of clinical practice." Those in the "Working on a Doctorate" group mirrored many of the comments described earlier. One subject valued the time to be able to work at home: "completed work is the key." Flexibility can take a different value to some faculty. "It is a benefit, but nursing has less flexibility than other faculty on campus. We put in more hours than other faculty and our credit load is not the same." Another faculty member noted that with the change of governance from one college system to another there was a definite change in the flexibility. She felt that there was more flexibility in the old system than the new. 
In the "Doctorate" category there are many of the same thoughts and feelings; however, one subject stated that it was a "perk of teaching. When in the hospital setting you have the clinical responsibility to the hospital the entire shift. Here there is some leeway." Likewise, "there is flexibility, but I am on call 24 hours a day to some degree. It can be a burden with professional responsibilities; they may overrule my personal goals."

Years of Experience by flexibility. In the "0-5" category, the flexibility is of value to the nurse educator. "I have a 9-month contract and summers off." The same person noted, "I will recoup benefits in the long term." For the "6-10" category, one member stated that, "it depends how you look at it. I can take home work when I want to; in the clinical setting I cannot turn it off. I think clinical nurses suffer from PTSS (Posttraumatic Stress Syndrome) at the end of a busy shift." In the "11+" category, one educator noted the degree of flexibility was where you worked. "I have worked at two universities and here it is great."

Size of College by flexibility. No differences in comparison beyond what was previously discussed.

\section{3.a. Flexibility?}

IS9. What is your sense of this flexibility now (as an experienced faculty member) compared to when you started? What was your perception of this at the time when you first started teaching? Was it influential in your decision to change your career? Why?

Overall the participants seemed to realize shortly after the initial employment that there were some significant differences in expectations. The responses from the subjects depict some of the frustrations they felt. Throughout the interviews the faculty were honest and provided a different picture concerning their expectations of the flexibility of the position. Of all the responses to this question, only one participant affirmed that it 
was what she expected.

Educational Attainment by changed perception of flexibility. The "Less than a Master's" and "Working on a Master's" groups responded to the question candidly. One noted:

When I first came everyone said it was very flexible but I had a little bit of rosecolored glasses on. The responsibilities of new faculty are such that I am so busy that I have to be here or I can't get my job done. I get home at 5:30 or 6:00 and I bring work home. The added load of advising on top of the new faculty workshop, I just didn't think I would be this busy.

Another nurse educator stated, "I started in the summer and it's a little different. It is slower and I did not have as heavy a load. I am still learning everyday about my job.”

The "Master's "category had very similar responses. "I thought it was going to be easy; it's not."Another indicated, "I didn't see it as real flexible, I was thrown into it. I didn't have a mentor."Another said, "I was unaware how time consuming it was. Other departments are not aware of the time spent in nursing." The "Working on a Doctorate" group talked about the difference between the clinical atmosphere and teaching. "When I first started I was on a rigid time clock behavior. There was a transition when I first started teaching. I would sit in my office all day even if there was nothing going on and slowly I realized I do have that flexibility." Another member of this group stated, "I thought, oh wow, 9-3--then I can go home. That was the image, not the reality. There is time needed to develop tests and lectures. Even though my colleagues were helping me I still had to learn a piece of that on my own. But despite all it still was more flexible."

The "Doctorate" group expressed their sentiments, "I had no idea of the amount of work instructors went through to teach. I prepared for my first lecture and it was to be four hours with the students. I gave it in 15 minutes. I did not know what to do with them after that." "I have more flexibility now, I have a greater expectation of myself and my 
students and I can prepare in less time." All of the respondents stated that this did not have an influence on their decision to change; however, two commented that they thought they had thoughts that they may have made a mistake.

Years of Experience by changed perception of flexibility. In the "0-5" year group the comments resonated much of the same feeling. One faculty member responded to the question by saying, “when I started, I felt like I didn't have enough time for anything. I thought it was because I did not have my Master's that I was not prepared. But, I was mentored and now it is easier. I have my lectures prepared." The "6-10" group had many of the same comments; however, one faculty member stated that, "I did it because I wanted to teach. When I interviewed they told me I would be busy and I am. My work ethic is to do a great job. It did not influence my decision to take the position." In the "11+" group one faculty member felt like her previous teaching job had more flexibility. "I don't know if it is the structure or the philosophy. Here at the college I have come to not care for the rules. I do not feel I am in a professional atmosphere. But there are some benefits to teaching."

Size of College by changed perception of flexibility. "Small" category faculty discussed the realization in the beginning that the job is very time consuming and a certain amount of adjustment was necessary in order to continue in teaching. "I thought it was going to be more flexible but we are required to do things in the community and committee work. It takes a lot of my time." One "Medium/Large" group faculty member stated a similar sentiment but focused on a different aspect: "I am more comfortable now in the role. At first, the learning process and the time spent prepping for lectures was time consuming."

\section{3.b. Collegiality?}


IS10. Can you compare the collegiality of your teaching position within the college setting and your previous clinical position? Do you consider this a benefit or a burden of teaching? Why?

Faculty across the categories had various opinions of the collegiality of the college setting in relation to the clinical setting. Certainly the support that the members received early in their teaching careers provided some positive perception of the process. One faculty member from the "Small" college group expressed a representative sentiment, "I have had good relationships with each of other in the clinical setting. In this setting [college] we do things outside of the work setting, we are more sharing of family." According to 53\% of the subjects, they had come from clinical environments that were very collegial and their responses reflected a somewhat neutral response.

Educational Attainment by collegiality. The "Less than a Master's" and "Working on a Master's" categories did not see it as a burden but did not see it much different than the clinical setting. "I have had a good relationship with all jobs I have had so I can't say it is a lot of difference." However, one faculty member was very positive, "nursing is a good team here; the Coordinator is great, and the college as a whole is supportive just like working on the floor."

Faculty in the "Master's" group determined that it was not a burden but not an advantage. "I knew I wanted to come to work each day and that was the driving force." Another faculty member referred to the morale of the hospital unit. "I felt very much accepted and welcomed and people have continued to help. It is not as hectic as in clinical and the morale was very low [there]. We still have stressors but it is different."

In the "Working on a Doctorate" group, responses tell of the sameness as well as the differences of the two environments. "I would almost consider it the same. My 
immediate clinical workers worked well together, but there is no time clock here." Another stated, "My previous job was in quality management and it was truly a team approach. Here it is about the same." "I think we have a lot of collegiality but I think it is the group of people. I have worked some places clinically where it was a good collegiality environment so I can't say that is an influence for me to teach or to be here. But I do value that here."

In the "Doctorate" group the responses are much the same. "I believe the collegiality is greater here than in clinical. In clinical one is focused on the task."Another responded, "It is a totally different environment than working in clinical. But there was a good relationship in clinical and it is not much different than teaching. I have been here so many good years and formed good relationships."

Years of Experience by collegiality. In the " $0-5$ " group the comments are much the same. One faculty member states that, "it is neutral one way or another." Of collegiality, another faculty member said, "No, I would almost consider it the same, my immediate clinical workers worked well together, there is no time clock now and it is a good working relationship."

In the "6-10" category, a participated indicated, "We get along pretty well, we are very busy. Despite faculty cuts, we all get along well. It is definitely a benefit. If you have a problem, you can talk to others."

One faculty member in the " $11+$ " category described the loss of some of the collegiality as the college changed systems of governance. "When I first started here I felt that you were more of a part of an academic setting. Now we are more like a business. Clinically there is a time clock; here there is no time clock, there is closure and we are trying to adjust to that." A very positive faculty member stated, "It had been of the best 
benefits that have come my way. But it is the people, I don't get along with everyone but it is like a sisterhood."

Size of College by collegiality. No differences in comparison beyond what was previously discussed.

IS11. What is your sense of this collegiality now (as an experienced faculty member) compared to when you started? Was it influential in your decision to change your career? Why?

Overall as a faculty, the responses conveyed some difficulty in the adjustment process as new faculty. Five of the faculty said it was influential in their decision to change their career choice, three verbally stated that it did not have any influence in their decision to teach. The existing atmosphere in which the new faculty member becomes a participant can influence the overall adjustment. Several faculty discussed what was in place in relation to problems within the program and their overall smooth adjustment.

Educational Attainment by perceived change in collegiality. In the "Less than a Master's" and "Working on a Master's" category the participants noted that for their nursing program, relationships were very positive. "I don't regret my decision to leave the clinical area. I still get the best of both worlds. I can take the students to clinical and yet I am teaching. It does not restrict me, it fulfills me." One faculty member had difficulty in the beginning focusing beyond what responsibilities were at hand. "When I first started teaching I didn't know that anything was outside of where I existed. I was so consumed with what I was doing. Things have changed now."

Faculty in the "Master's" group reported that the collegiality when they first began their teaching careers was not what it is now. "Never did I think the collegiality would be like it is now when I first started teaching. There cannot be any group of women anywhere that's anything like we have."Another stated: 
There is a big difference in what I experienced when I first started teaching than what I experienced in the clinical setting. When I first started there were only five of us and we were a very close knit group. Now we are a much larger group and I don't feel as informed of other teachers with regards to what they are doing, but I think that we still remain pretty close knit and try to keep up with what is going on.

The "Working on a Doctorate" category faculty discussed the problems of the relationships in their college when they first arrived. "When I first came here there were four courses taught and four silos. We were all separate and now with new faculty, a new coordinator, and focusing on our curriculum we are working together."Another participant said, "When I first started teaching here the group was not supportive. Now they are gone and I feel that my opinion is sought out and respected."

In the "Doctorate" group there were two faculty who were outspoken about their adjustment to the teaching milieu. "I came as a novice, but I had experience , I wasn't green. They don't trust you until you show them you can do your job. I am part of the group now." Another faculty noted, "In the last clinical setting there was much more closeness and department parties. At the past teaching position there was no collegiality and I left."

Years of Experience by perceived change in collegiality. In the "0-5" category, responses were positive for four out of the seven faculty. For example, "I have gotten to know the faculty and they have warmed up to me. I think there is more collegiality now than before, I didn't know how that would be."

In the "6-10" category the five faculty responses were similar to the others. One faculty member discussed the difficulty in letting go. "I did not want to let go, I wanted someone to stay with me at all times. You have to cut that umbilical cord and get out on your own. I wish sometimes I had more under my belt." Another responded," I was 
totally green and not aware of anything. Schools of nursing should have mentoring for new faculty. You can graduate from the best Master's program and reality sets in."

Faculty in the "11+" group were both positive and negative in their responses about the collegiality in their institutions. One subject whose college had undergone a governance change and had different department leadership explained that, "The atmosphere has changed from the beginning." The changes in leadership had impacted the morale of the nursing faculty.

Size of College by perceived change in collegiality. No differences in comparison beyond what was previously discussed.

"You have to love it, you either do or you don't. I changed 32 years ago. Teaching is in my blood, I probably have rocks in my head."

3.c. Organizational climate?

IS12. Compare the organizational climate of your teaching position within the college setting and your previous clinical position. Do you consider this a benefit or a burden of teaching?

Nursing faculty responded in agreement in some cases that the organizational climate of the college setting is very positive. Some compared the autonomy of each setting and provided insight into that aspect. The view of management's role in the comparison is also discussed. Twelve of the 19 faculty regarded the organizational climate in the college setting as a benefit to teaching.

Educational Attainment by organizational climate. In the groups "Less than a Master's" and "Working on a Master's," all three faculty saw the college organizational climate as a benefit of teaching. One faculty member noted that she was in a fairly autonomous clinical position in the hospital but decisions came through the physician in charge. The autonomy the nurse educator experienced was experienced within the 
semester courses. Although responsibility for decision-making is within the team of faculty teaching the courses, there is a sense of being in control of the course and program. Another faculty member remarked that in the college setting supervisors have been very "hands-on" and the faculty works together despite "differences in personalities." "We complete the puzzle."

In the "Master's" category one faculty person had worked in a hospital in which nurses had to be part of a union. The union officials made decisions for the nurses. Another faculty member talked about the contentious nature of the relationships in the hospital. "I worked in a hospital where it was 'administration versus staff" Managers managed from a distance. Here we have input and there is more support here."

For the group "Working on a Doctorate," one faculty member addressed the question by responding that, "Faculty drive the curriculum whereas, in the clinical arena upper management make the decisions." In her setting at the college, the amount of committee work is greater than in the hospital.

The "Doctorate" category member could not compare the teaching versus clinical organizational climate. "It's apples and oranges, I can't compare them. The organization is different as far as outcome. The hierarchy is very different in the clinical setting. The chain of command is more linear and autocratic."

Years of Experience by organizational climate. In the "0-5" group one participant responded that, "the coordinator is very supportive of the managers in the clinical setting. Clinical managers do not have the same feel of a unit like the program coordinator had of the nursing department. At the hospital we could struggle and they would not get it." Five of six faculty responded that the organizational climate in education is a benefit to teaching. According to participants in the "6-10" group the organizational climate is a 
benefit to teaching and the work in the college setting is more autonomous compared to clinical. Three faculty out of the five felt it was a benefit to teaching. In the " $11+"$ category there were five subjects who responded that the organizational climate in teaching was a benefit. One faculty member described the climate in the private not-for profit hospital where physicians are very revered and there was very little autonomous decision-making by the nurses. She noted that the organizational structure of the college had changed as well.

Size of College by organizational climate. In the "Small" category, five faculty out of the seven who answered this, noted that organizational climate was a benefit to teaching. In contrast the slit was more even in the "Medium/Large" category 6 of 12 faculty acknowledged the organizational climate of the college was a benefit to teaching. One said, "I have a better sense of organization, all roles of administration."Another added, "I think I am more aware of the process of the University now. I have served on the Faculty Senate the last three years and it has been very helpful to me." Five faculty did not think it was an influence, and one did not know, "It goes back to there were several things I did not know."

IS13. What is your sense of this organizational climate now (as an experienced faculty member) compared to when you started? What was your perception of this at the time when you first started teaching? Was it influential in your decision to change your career? Why?

Responses from the entire faculty seemed to vary according to what the atmosphere at the college was or is currently. Seven faculty answered that the organizational climate at the college was not influential in their decision to teach. Four faculty responded that it did.

Educational Attainment by perceived organizational climate. In the "Less than a 
Master's" and "Working on a Master's" faculty groups one faculty responded that the "good decision-making as a group influenced me that this would be a positive move." Another noted that her "supervisor was in touch with what the faculty was doing." She reported that they faculty as a whole meets on a daily basis each morning to decide who needs help.

In the "Master's" group one faculty member stated, "At the time I came here to teach I was impressed with the reputation of the nursing program." Another faculty member stated, "When I came I went there with rose colored glasses on. In my previous position I was pampered". Another faculty member talked about the change in governance. "It was under UK and that was wonderful. The [current] system has not done a good job of developing a career ladder. I see a very big difference."

In the "Working on a Doctorate" category, one faculty member stated that "the organizational climate is much better now." The nursing program had been under scrutiny for its poor NCLEX [National Council Licensure Examination] results and a resulting Kentucky Board of Nursing investigation on the management of the program and the teaching practices. "That was the wake-up call and the turning point." The nurse educator likens it to a "Perfect Storm." With a new coordinator and a new Dean she says, "They have really brought things together. We knew where we needed to go, we just did not have a path to really get there."

Years of Experience by perceived change in organizational climate. In the " $0-5$ " category, three of the seven faculty in this group reported that the organizational climate was an influence on their decision to teach. With more experience came a greater understanding of the college and its working relationships. One noted, "When I started I realized who I would be reporting to and that we could have a working relationship and 
we could exchange ideas. I could feel I was going to be supported".

In the "6-10" group, two of the six acknowledged that the organizational climate did have an influence in their decision to teach. Changes also occurred in relation to faculty or administrative changes. One person noted:

When I first came there was a very aggressive relationship. There were differences of opinions and leadership style. There was also some inflexibility. The manager was not in the same building and it was old thinking. With new leadership we are together and integrating ourselves in the work of the college.

Another faculty member discussed her experiences with administrative confrontations.

"When I first started teaching, within 2-4 weeks the faculty gave a vote of no confidence to the President. I wondered if I did the right thing."

In the " $11+$ " category, only one faculty member responded that the organizational climate was an influence on her decision to teach. The other five subjects noted that a good organizational climate is important in the overall working of the college, but it would not affect their decision whether or not to teach:

I did not know enough about the administration of the college to have it affect me. I am learning about it more as it changed. Things are much different now than when I started. The money situation and the students are different. It must be OK, I've stayed. I really don't want to go back to the clinical setting.

Size of College by perceived organizational climate. In the "Small" college category, none of the seven subjects were concerned about the organizational climate influencing their decision to teach. In the "Medium/Large" group, three faculty noted that the organizational climate of the college at the time of their hire did affect their decision to teach.

\section{3.d. Prestige/status?}

IS14. Can you compare the prestige/status of your teaching position within the college setting and your previous clinical position? Do you consider this a benefit or a burden of teaching? Was it influential in your decision to 
change your career? Why?

Overall, the discussion concerning prestige/status yielded a variety of responses. Eight faculty acknowledged that there was a certain amount of prestige/status to the position. Three said no and the other eight did not respond. One nurse faculty member states, "I'm not one on prestige, I consider being a nurse more prestigious than a faculty member."

Educational Attainment by prestige/status. In the "Less than a Master's" and "Working on a Master's" groups one member admits that "I feel a sense of pride just being a faculty member." Another faculty member answered that she "guessed that prestige and status depends on who is looking at the position." In the "Master's" category two faculty discussed the meaning of prestige/status within their college. "I would consider this like prestige. You feel more value for what you bring to education." On the other side, "I don't think there is much prestige here; otherwise I would not have to work so hard."

Two of the faculty from the "Working on a Doctorate" noted that the status of the job was influential in the decision to teach. One participant said, "I don't want to discount being a staff nurse, but I think this is a prestigious job. Compared to my other role, it makes me feel good and I am moving up." Another group member said, "Status did not influence my decision but I think this is a prestigious job compared to my other role." A faculty member pointed out the negative impact of being a nursing faculty member in a community college. "I don't consider this a benefit. When taking students to clinical I am not respected by the staff. I am now working on a $\mathrm{PhD}$ and when students [other graduate] in my class hear that I teach at a community college they make derogatory comments about teaching in an associate degree nursing program. 
In the "Doctorate" category, two of the faculty admitted that it influenced their decision and the other two did not. Likewise, three of the four thought there was some prestige/status to the job. The faculty member who did not, answered the question similar to the previous faculty member in the "Working on a Doctorate" group. "It can be a negative in higher education circles if teaching in an associate degree program." Like the faculty member in the first group, it may depend on who is looking at the position.

Years of Experience by prestige/status. In the "0-5" group, four faculty out of seven responded that the job had prestige/status. Three faculty answered that the prestige/status of the job had some influence on their decision to teach. "I would consider this like prestige. You feel more value for what you bring to education. I totally appreciate what we are preparing to be a bedside nurse. There may be more prestige when you take the students to clinical."

In the "6-10" category, three faculty said it was influential in their decision to teach and the other three said it was not. "I would say that it is influential in changing my career. It is a benefit being associated with the department."

In the "11+" group two out of five responded that it was an influence on their role. The other three did not feel there was much prestige or that it influenced their decision to become faculty.

Size of College by prestige/status. In the "Small" group category, two out of seven faculty answered the question that yes, there was prestige/status within the college. The other five responded in a variety of ways. "I have adjusted to it. A lot of times the community will make a difference, 'Oh you teach nursing?' They may see it differently; I still see it as nursing."

In the "Medium/Large" group, four faculty responded that it influenced their 
decision to teach, five said it did not, and three did not answer. "With the prestige, I think community members look at you as preparing the nurse of tomorrow. The faculty member is looked upon as prestigious in the community." Another faculty member took another view. "I have never really thought about prestige and status of my position. I had some prestige and status in my clinical position, it was a very select group and there was status working there."

IS15. What is the sense of this prestige/status now (as an experienced faculty member) compared to when you started? What was your perception of this at the time when you first started teaching? Was it influential in your decision to change your career? Why?

Six faculty in the entire group responded that from the beginning of their employment as faculty the prestige/status of the position was influential in their position. For several faculty members, their perception changed after they first started teaching; for others there was not a perception or change. In one faculty member, her response was that her prestige would be less because she moved to an associate degree program from a baccalaureate program.

Educational Attainment by perceived change in prestige/status. In the "Less than a Master's" and "Working on a Master's" groups the faculty's perception of faculty changed. "My perception has changed somewhat. Students don't realize the time and effort faculty are putting in. They don't see the effort; I have a different appreciation now." Another remarked, "I don't think I remember as a student how wonderful faculty were; they are the authority."

In the "Master's" group there was one member who did not understand the process. "I really didn't understand the status thing, I was kind of floating around and trying to learn. I wasn't into any prestige.” Another participant's view of prestige/status 
did not change.

In the "Working on a Doctorate" group, three of the five subjects in this category did not view prestige as a positive. "It wasn't on my radar." Another faculty member stated, "I never did think of that at all."

In the "Doctorate" group there were totally different responses from each one of the group members. A positive one, "I think when I first started I felt like there was a prestige already about being a faculty member." On the opposite side, "I had no perception."

Years of Experience by perceived change in prestige/status. The faculty in the " 0 5" year group, four of the seven subjects answered positively that the prestige/status was influential in their decision to change careers based upon their perception when they first started teaching. One noted, "I thought of the status before I became a teacher. I liked the hours, the setting, and the different pace."

In the "6-10" group, three faculty were not interested or it was not a factor for them. One thought the prestige was in place prior to his employment. One implied that prestige had to be made by the individual. The last member of the group responded to the question with a concern that the prestige/status within the nursing program would be gone. 'Some people don't even realize there is an ADN program here that will soon be engulfed into the school of nursing. The history of the program will be lost."

In the "11+" group, two faculty responded that it was not influential in their decision to change careers, two faculty had no perception, and two thought there was a change in perception from the beginning of their teaching career. "There is a burden attached to that [status of being an instructor]. You are expected to present material to all different levels of students. You are expected to come up with perfect test scores. Some 
of it you can't do."

Size of College by perceived change in prestige/status. Other? No differences in comparison beyond what was previously discussed.

3.e. Other?

IS16. Can you think of any other factors related to work environment that influenced your decision to change from clinical nursing to faculty status? How and why?

Across the groups the faculty discussed the physical changes of their bodies that may have guided them to teaching. For many, the 12-hour shifts and the constant stress of the unit as a bedside nurse was a reality that soon encouraged them to seek another career but to remain in the nursing profession. For many, the love of learning and being with students was a factor that influenced their decision to change. One faculty member from a "Small" program put it this way: "As a staff nurse I loved working with students. I worked harder when students were there. I wanted to teach them and give them experiences I thought they would need." For several, the flexibility was again part of the discussion and to have a family life as well as teaching worked best for them.

Educational Attainment by other. In the group designated "Less than a Master's" and "Working on a Master's," flexibility was important for one faculty member. "My biggest deciding factor was home life and time with family." From the hospital to the college setting the change in schedule and the flexibility to be a greater part of family life was important. Another faculty member answered the question by saying, "I enjoy working with students and I enjoy the flexibility." The other faculty member in the group was pleased with the control that faculty have over the syllabus and the coursework.

The "Master's" group responses focused on flexibility, love of learning, and the physical demands of bedside nursing leading them to inquire about teaching. "I just love 
to learn, I learn every day." In relation to the physical stress, "I think that just the physical stress, I had a lot of responsibility on the unit I worked and it was physically demanding. The physical demands are much less here and that was a big factor."

The "Working on a Doctorate" group told of their love of teaching, the physical demands of bedside nursing and working with students. "I think I realized that the 12hour shifts on your feet were difficult. The long hours of working double shifts, and I realized how dangerous it was." Another faculty member had the same ideas. "My age, my physical condition, my joints." Other faculty mentioned their work with students, "I like the idea just being with students, I like seeing the light bulbs go on." Another faculty member's responses; "When I worked at the hospital I precepted students. I think it helped me to see this as something I would like to do. I liked teaching them and watching them grow. It makes me feel good."

The "Doctorate" group responses were much the same. The flexibility was important as well as the opportunity to interact with students. Two faculty member's responses centered on the aging group of nurses and the opportunity to be in where a less continuously stressful environment would be. "The flexibility of being able to continue my education, refine my knowledge and have the opportunity to interact with students, educators, and influential constituents in the community are rewarding." Another faculty member cited," I think it was a transition from knowing that I am not always going to be a bedside nurse all my life, made me position myself, and teaching is where I landed." Years of Experience by other. In the "0-5" group, the three faculty's responses centered on the flexibility of the job and teaching students. For "6-10" group, two faculty responded to the physical stress of the hospital setting while three faculty's responses included the love of learning and being with students. "It's a mental challenge, it's all 
about helping students." Finally, the " $11+$ " category responses were similar to the other two groups. "I felt like I was getting older and could not work 12 hour shifts. When I first came here and did clinical I had pitting edema, I thought I couldn't do this." On the topic of learning, "Especially at the beginning I loved learning. The constant learning of something everyday just amazes me. I would not have done this as a staff nurse."

Size of College by other. No differences in comparison beyond what was previously discussed.

\section{Research Question 3 Summarized}

When faculty was asked to compare the flexibility of the teaching role with the clinical role, the responses were positive and very enlightening. Recalling their days as new faculty, they described the reality of the first months of their teaching career. For many of the nineteen subjects, the transition was not smooth nor was it always in a forward direction. For some of the participants the organizational climate was in turmoil as administration and in some cases the very bodies that govern them were changing. From this question the main ideas identified were: (a) flexibility in the college setting is one of the key indicators identified by the nurse educators for changing careers from the clinical setting to academe, (b) the success of role transition from the staff nurse to the faculty member is centered around faculty support, and (c) the love of learning and interacting with students is an important part of the nurse educator's role satisfaction.

\section{Research Question 4}

To what extent are pedagogical considerations influential?

The participants responded emotionally when stating how they felt when they taught. Faculty responses included feelings of empowerment, excitement, enthusiasm, and of being energized. None of the faculty responded negatively. A few indicated they 
were tired and the responses from the " $11+$ " nursing faculty experience group were the least positive when the subjects responded to rewards of teaching.

4.a. Love of teaching?

IS17. Think about your reasons for changing from the clinical environment to teaching. How do you feel when you teach?

Across all of the groups, the faculty described emotionally at times how they "felt" when they taught. As the nurse educators put into words what it meant to them, the body moved, the hands gestured, and the words were truly full of emotion. These nonverbal cues were a testament to the depth of these responses. The personal control seems to be important. A participant from a "Medium/Large" college noted that "Even though we team teach I feel I have control over my content. I feel ownership when I go into the classroom."

Educational Attainment by perceptions of teaching. The "Less than a Master's" and "Working on a Master's" groups were very positive in their description in how they felt when they taught. "I feel empowered. I feel that when I teach I have the knowledge they need." Another faculty member stated, "I just lectured today and I feel more in control of the situation. But, sometimes I still feel more in control in clinical." A third faculty member stated," I like teaching. I like getting up and explaining content."

In the "Master's" group one faculty member explained it as," it is almost a high. When you are trying to make a point, students look at me in amazement that I have all this knowledge that they need to know. I tell them, people, it's years and years of experience." Another participant answered, "I have a lot of energy. I love our profession and the knowledge. Knowledge is power."

In the "Working on a Doctorate" group one subject said, "I love it--absolutely 
love it. When you talk about something and they respond and you see the light bulb go on." Another faculty member stated, "I am charged, maybe a little too much. I like teaching, I wasn't comfortable at first, but I enjoy taking the knowledge and putting it into language they can understand."

In the "Doctorate" group, one faculty member responded, "I feel excited, I feel comfortable, motivated, then accomplished. I feel very proud to share my knowledge with students." Similarly another stated, "I really like to lecture. I do entertain and I am a pacer. The students like to hear my stories." This subject became very vocal and waved her arms and followed her statement with a hearty laugh.

Years of Experience by perceptions of teaching. In the "0-5" group one faculty member talked about how stimulating lecturing is for her, saying, "I learn from the students. I learn when I prepare to teach them. When I complete it I hope that it is a nice satisfying feeling that some learning has occurred." Again, another faculty member described her feelings about teaching and the use of simulation: "I enjoy the classroom. I like to interact with students. I like watching them grow." She explained that the program has simulators to enhance the clinical experience of students. The simulator becomes so much a part of the student and their learning that the simulator is introduced to the family at graduation.

In the "6-10" group, faculty responses to the question continue to mirror those of the other group. "I get a sense of satisfaction. I like to see learning take place. You realize your reason for changing from clinical to teaching." For some faculty the preparation for lecture or clinical provides additional rewards. "I feel a lot better about my skills and nursing. I have more knowledge now than I ever did in clinical practice. I enjoy trying to find ways to explain complicated information to the students and they get it." 
From the faculty in the (11+) group, one stated, "I feel like I am promoting a new person learning nursing science and that makes me feel good." Feeling good about student learning as well as themselves is common among the faculty. "I like to teach. I feel good especially if I am familiar with the material and have experience. I can give back to the students, I feel good about it."

Size of College by perceptions of teaching. No differences in comparison beyond what was previously discussed.

IS18. When you started your career as a nursing faculty member, did you see teaching as a plus or a minus? Why?

Overall the faculty were extremely positive in their responses to the question. Across the groups there were three faculty whose responses were less positive. Those will be included in the grouping which they represent.

Educational Attainment by teaching as a plus or minus. In the "Less than a Master's" and "Working on a Master's" groups, all three saw teaching as a positive. One faculty member shared," I enjoy teaching and see it as a plus." Another said," I felt like I haven't been a nurse for 50 years but I feel like I can share my knowledge. I realized that I was learning a lot as I go.”

The "Master's" group faculty noted that it was a plus in each of their responses except one. "It is not a plus or a minus; I would see it as an extension of my clinical nursing only on a different level." A faculty member was extremely proud of being part of the educational process. "It was a plus that I have the ability and was qualified to teach and to help students."

The faculty in the "Working on a Doctorate" group shared that teaching was well perceived. One faculty member saw it as positive in relation to her own place in the 
profession. "In saw it was a plus in relation to flexibility. I felt like I was actually a step up in my career. Even though the pay did not reflect that, I felt like I had moved up to where a Master's level nurse should be."

In the "Doctorate" group the faculty member responses are somewhat pragmatic. Three responses out of the four had more to do with the faculty member in relation to the college. "It is a little hard for me to answer that. I took this job and enjoyed it so I did not have any preconceived ideas of it being positive or negative." A second faculty member weighed in, "When I started teaching the anxiety of it was I would say was neutral to me. I am different; I did not notice it." "Probably the only thing I saw was I was off during the summer. I have a farm and had to do a lot of work on the farm. I had to supplement my income with extra jobs."

Years of Experience by teaching as a plus or a minus. Of the seven subjects making up the " $0-5$ " group, six felt that teaching was a positive, and one was neutral. One faculty member stated, "I see it as a plus that you are influencing these people who are going out and taking care of people. When I see that Johnson and Johnson nurse educator thing--I really am making a difference."

In the "6-10" group, five of the six faculty reported that teaching was a positive factor. One faculty member stated that she was glad to have the opportunity to give to the students. "I felt like I had something to offer for training nurses. I felt that I could be supportive of students and I felt that I could use my management background without necessarily supervising." Another faculty member responded, "I saw it as a plus, the opportunity to continually learn and make a difference within the individuals' lives and to assist them to be productive members of society."

In the " $11+$ " group, four of the seven responded that teaching was a plus. One 
faculty member saw the clinical element of teaching as positive. "I like the clinical hands-on part but you also have to give them the rationale." Another nurse educator responded that," I think it was a plus, I would not have stayed."

Size of College by teaching as a plus or minus. In the "Small" college group, one faculty member included a response more far-reaching, "I have graduates that have gone to many states and I've had the opportunity to where I have been in the hospital and I have had a graduate taking care of me and to give me an injection." Overall, this group of eight sensed that there was a tremendous amount of pride and of success when seeing the students graduate or view them caring for patients in the clinical setting.

In the "Medium/Large" group, one faculty member saw teaching as positive and that "I think it was from the role models I had while I was in school. I looked up to them." Eight other faculty members in this category also regard teaching as a plus. There are three members of this category that saw it as neither a plus nor a minus.

4.b. Lifelong learning?

IS19. Talk about the extent that you have always loved or disliked school. Did this have any influence on your decision to enter teaching? In what way?

Seventeen of nineteen faculty responded with some kind of positive response either that they enjoyed reading or they loved learning. Many of the faculty stated that it was somewhat influential in their decision to enter teaching. Typical comments from across the 19 respondents were the following. One faculty member added that there were some required courses that she could not understand the rational for taking. But she said, I like courses that she could no understand the rationale for taking. But she said, "I like learning and challenging myself." Another responded that she too enjoyed learning, "But I became tired of school and its demands. It did influence my decision to teach. I like to 
see the learning, the whole process." Another added, "I always enjoyed school a lot. I enjoyed learning. I guess it did influence [my decision]. I enjoy teaching and being in the atmosphere." Similarly, "I always liked school. I am not sure that it had an influence on my decision to teach but I personally always liked school." Typical of many faculty was the response, "I always did well in school. It was a sense of pride and accomplishment." Educational Attainment by love of school. Two of the three faculty in the "Less than a Master's" and "Working on a Master's" groups liked school. "I came home from school and played school. I always liked learning and challenging myself." The second faculty member responded to the question, "I think I will always be a professional student. I am always reading something and I like taking classes." The third member of the group describes a "love-hate" relationship with school. "I do really enjoy learning, it is just the time involved. I don't have the time."

"Master's" faculty all acknowledged that they liked school. "I love to read, I would read to my dolls. I never dreamed I would be standing in front of people teaching. Education allows power." Another faculty member responded that she would be learning at any age. "I love to learn. Probably the biggest impact on my changing [careers was that] I always loved school. I don't think I will ever be too old to take classes."

All five of the faculty in the "Working on a Doctorate" group answered that they liked school. All are enrolled in doctoral programs and the pursuit of their own education lends credibility to their responses. "I have always enjoyed school, although I did not think it was difficult, it was time consuming." Another student's response is similar, "I always enjoyed school. I keep going back, I don't know what I will do when I finish." All four of the faculty in the "Doctorate" group replied that they too liked school. "I've always loved school and have continued to earn several degrees. This has certainly 
influenced my desire to teach. I can relate to students as I face the same challenges.” Another faculty member responded, "I always loved to read. I've always been a vociferous learner. Give me a book and I can learn.”

Years of Experience by love of school. No differences in comparison beyond what was previously discussed.

Size of College by love of school. No differences in comparison beyond what was previously discussed.

4.c. Possibility of influencing the next generation of nurses?

IS20. How would you describe the rewards of teaching? Has your sense of this changed since your started this career? In what ways?

This was another question in which all faculty across the groups responded with very similar thoughts and ideas. Some responded that they were more satisfied when in the classroom. The student was able to understand the information. Others were rewarded when they encountered a graduate nurse working in a nearby clinical setting. For example, faculty noted the following, "The rewards of teaching are the success of the student. Ten years from now are they still in nursing? Do they still love it?"Added another faculty member, "I would basically see the reward if I am able to share what I know with others and I see them being so eager and then wanting to do the "hands-on care' and the initial excitement." It's fulfilling for me, it's a positive. Even with a PhD I want to stay in the classroom as well as do research." Another offered, "It is rewarding when I see them caring for others. I think that is the greatest gift." Finally, one faculty member included the satisfaction that the faculty member gets when words of appreciation are spoken. "You do not get 'I appreciate you' when you work in the clinical setting....Nursing is not glamorous; you have to have it in your heart." 
Educational Attainment by rewards of teaching. In the groups "Less than a Master's" and "Working on a Master's," one faculty responded, "The rewards I see are when you see the students learn, when the light bulb goes off, I get it." Another faculty member stated that, "When they come back in and thank you, this is the reward to see them attain their goals and [become] a successful member of the community."

The faculty in the category "Master's", identified two thoughts similar to those in the earlier group. Some faculty were rewarded with the performance of graduates in the clinical setting while others focused on the immediate classroom reward of the student response to understanding a theory or topic. "I get all choked up at graduation and pinning. When they greet me as nurses or have gone on to higher education, that makes me feel good just to achieve and I have had a part in that."

The "Working on a Doctorate" group identified three areas of satisfaction. Another faculty member responded that the growth of the student's knowledge and skills from the first course until the last course. "I started in an earlier course and now I teach in the last course, I see students putting it all together." The last faculty member identified that, "[t]he biggest reward for me is when the nursing student group comes in and they all leave. Not everybody can be a nurse but everybody can graduate."

The "Doctorate" group subjects answered the question in much the same way as those in the other groups. "When you can see it in their eyes, that's the big reward for me." Another faculty member noted that, "[t]he rewards of teaching include knowing you have done your best to impart knowledge and to see them grow. Every day I take students to clinical and I see former graduates and that makes me feel good to see them functioning effectively."

Years of Experience by rewards of teaching. No differences in comparison 
beyond what was previously discussed.

Size of College by rewards of teaching. No differences in comparison beyond what was previously discussed.

IS21. How do you feel about your relationship with your nursing students?

Faculty members across all of the groups responded to this question with concern for establishing a professional relationship, yet developing the closeness to be approachable to meet the students' needs in the clinical or lecture setting. The faculty in the study described their concerns with today's social media sites. The maintenance of policies and guidelines for students was a major concern for this group of faculty. Several faculty members in the study verbalized maintaining a relationship with the student while serving as a professional role model. "I tell them come to see me if you are struggling. Sometimes I have to go get them." Another stated, "I have high expectations for them and often it is difficult for them to accept that. We have a mutual respect for one another."

Educational Attainment by relationships with students. In the "Less than a Master's" and "Working on a Master's" groups one faculty stated, "I try to be fair and consistent and I want to be that instructor that is easily approachable." The two other faculty members making up this group related their concern about maintaining a professional friendship with the student. "I think I have a good relationship with the students, but it is hard sometimes to keep professional."The other side, "I try not to do that friendship- teacher relationship. I try to be that role model for them instead."

In the "Master's "group all faculty believed they had a good relationship with their students. "I think I have a good relationship. I have a smaller group of students for clinical and they are closer to me, but I think they can always come to me."Another 
faculty stated:

I think there are boundaries in teaching and interacting with students. They know that I keep an open door policy. They can come to me at any time and e-mail me or whatever any form or communication. You know I give them my home phone, my cell phone and my e-mail, they do not have to have a scheduled meeting to see me.

The faculty members in the "Working on a Doctorate" group were very careful to maintain the professional relationship with students. "With the students I learned very quickly to maintain a professional relationship, especially in today's social networking society. I am not your friend, I am your instructor." Another aspect of the relationship was provided by this faculty member. "Generally we have a good relationship. My door is always open. It used to be I am the teacher, you are the student, you must learn. Now I am the facilitator, you are the learner, we will collaborate together."

In the "Doctorate" group, the faculty provided two additional viewpoints on the student faculty relationship. "I think sometimes I intimidate them. I feel pretty good about the relationship. In lecture I don't visit. In clinical they like me better one-on-one. I do ask a lot of questions." Another viewpoint," I feel my relationship with students is a little different. Once students get to know me they are good with me, but my reputation precedes me. I am hard-nosed and have 'house rules.' I do not enable."

Years of Experience by relationship with students. In the " $0-5$ " group, additional viewpoints on student relationships included, "It is good, and I have not had any issues. I try to serve as a professional role model by setting standards. I communicate with them and hold them to it." One added a viewpoint on policies, "I feel pretty good with my relationship with students. What you see is what you get. I am fair and policies are policies and they get enforced equally. They want to know if anyone cares for them."

In the "6-10" group, additional viewpoints include, "I think it is alright, I guess. 
They have a good relationship. Very young students see us out to get them. Today a student told us they were glad we treated them as adults. You get what you expect." Another instructor compared the relationship with the student to the relationship with the patient in the clinical setting. "The student relationship is very multi-faceted. You get to know them personally more, but you don't do that with your patients, why your students? Sometimes you are their mother; sometimes they need a kick in the pants."

In the "11+" group, a faculty member responded, "I love them. I usually have a good relationship. They feel pretty free to talk to me. I am approachable. I usually get pretty good evaluations." Another faculty member responded, "I feel I am compassionate yet I can be very strong to support the program."

Size of College by relationship with students. No differences in comparison beyond what was previously discussed.

IS22. To what extent are these feelings connected to the immediacy of the classroom? To long term issues like the nursing shortage? Other?

The subjects in this study were focused on the immediate concerns of the student. Many of them noted that the nursing shortage was in the back of their minds, yet the development of a caring, efficient, and highly skilled nurse was their main concern. This comment from a faculty member in a "Small" college reflected the overall theme that the immediacy of student relationships was the driving force,. "I like to see the 'aha' moments. It is very challenging teaching, but co-instructor cohesiveness is important. Our goal is to work together for the success of the student. If they don't make it, we have met them half way."

Educational Attainment by time perspectives. The faculty members in the "Less than a Master's" and "Working on a Master's" groups were focused on forming the 
relationships with the students and then the rewards of graduating well-prepared novice nurses. "Being a new faculty member I am concerned about forming those relationships that are rewarding. To me, being in the classroom and them being comfortable talking with me is my concern." In relation to the product that is being prepared, "There is a rewarding sense that we're getting people out there. But I am hesitant. Are they really ready to take care of me?"

In the "Master's" group the faculty members were more focused on the preparation of the graduates. "Being in the classroom is my reward. I am concerned about the nursing shortage, but I am also concerned about the quality of graduate." Another response from a faculty member, "I start out thinking about the nursing shortage but just developing these relations and see them interact with their patients. That is the immediate reward, the warm and fuzzy feelings."

The "Working on a Doctorate" group also had the immediacy of student success in mind. "I think it is that relationship. I know in the back of my mind my contributions to helping the nursing shortage, but I see it more short term. It is more about helping them achieve their goal." A different perspective came from another faculty member: "I think that in the long term issue, seeing them out in the workforce and doing well is important. You don't see the immediate reward, it is more long term."

In the "Doctorate" group, one perspective of the faculty members was, "I think it would be both in the classroom and outside and a personal satisfaction as well as a professional one. I am contributing to the creation of a nurse that will take the place of one retiring. One replacing another on the front line of healthcare." Another response to the question of rewards and relationships would be related to touching the lives of the students: 
I enjoy teaching because it is a creative outlet for me and then the esoteric piece, if I touch one person in class, it touches five people that day and it touches 25 people later that day. I feel that it is a network. I will have impacted so many because I was able to share what I have. So that is my reward. I never connected it to the nursing shortage.

Years of Experience by time perspectives. In the "0-5" group, the faculty responded to the immediate rewards of the classroom and as a more global concern, the nursing shortage. "It's teaching. I like the didactic. I am in clinical putting theory into practice and I try to hold them [students] to certain standards. They may care for me some day."

In the " $6-10 "$ group, the faculty responded to the more immediate concern of preparing the graduate. "I really don't think about the nursing shortage. I think that if we keep plugging away we can resolve it. I enjoy the teaching part. I hate tests, they do too. I enjoy trying to get students engaged and learning."

In the " $11+$ " group of faculty, the concern for preparing the graduate was the primary focus. "I am an advocate for the students. We are not seeing that type of shortage in our area. I worry about quality graduates. Ultimately I want to focus on getting a novice student nurse through the class and getting them prepared to enter the workforce. We produce novices, hospitals want experts."

Size of College by time perspectives. No differences in comparison beyond what was previously discussed.

\section{4.d. Content knowledge and skills?}

IS23. How do you view the intellectual challenges of staying current with the knowledge about your field? Did this have any influence on your decision to enter teaching? In what way?

The research subjects were very cognizant of the need to stay current with the knowledge in their field. There was only one faculty member who acknowledged that 
staying current influenced the decision to enter teaching. "I want to be in situations where I am always learning. My body may fall apart, but my mind will be intact and I can talk about the current things and the new things that are out there in the field are stimulation." Another said, "Nursing is a field you constantly have to learn. You become stagnant if you stay with the knowledge from when you graduated."

Educational Attainment by maintaining current knowledge. The group of "Less than a Master's" and Working on a Master's" related that it was difficult to stay up to date with nursing and medical knowledge. One faculty member stated," I try to stay updated with drugs and treatments and I try to go to workshops, anything to maintain current knowledge. Other faculty in these groups reported reading journals to stay current. The entire faculty in these two groups (3) agreed that it did not influence their decision to teach.

The "Master's" group faculty said, "We just can't teach what we taught 20 years ago. It's not there anymore, things change." One of the newest faculty members in the group reported, "Sometimes trying to keep up with where you can tap into resources and technology and then give the students the most current information more than the textbooks is a lot for me to take in this first semester. Every day I pick up a little more information."

In the "Working on a Doctorate" group the faculty members' responses to the question were similar to the other groups. "We try to keep up and help each other. If we see something new we will tell everyone else. I can't keep up with all the clinical stuff." A faculty member reported the importance of staying current especially in light of evidence-based practice. "Nursing is always changing. One has to continually read and research, especially with evidence-based practice." 
The "Doctorate" group reported the same difficulties in staying current. "This is what it is all about. I want to be in a situation where I am constantly learning. I don't think it had a conscious part in my decision to teach." Likewise, another faculty member welcomes the challenge. "I think it is difficult to keep up with the knowledge required of the nurse educator. It has influenced my decision to teach.”

Years of Experience by maintaining current knowledge. No differences in comparison beyond what was previously discussed.

A faculty member from the " $11+$ " group remarked that, "Staying current is a challenge. There are so many things changing, even our textbooks. I have to find other resources."

Size of College by maintaining current knowledge. No differences in comparison beyond what was previously discussed.

IS24. Can you think of any other factors related to pedagogical considerations that influenced your decision to change from a clinical nurse to a faculty member?

Not all faculty contributed answers to this question. Those that did provided insight into their enjoyment of hands-on teaching in the classroom and clinical setting as well as having the opportunity to teach. Typical of comments from across the 19 respondents were the following. "Society as a whole is more in tune with what is going on (patient kept a journal). I wanted to be the patients' advocate when I was in the clinical setting. I was on the job 24/7. I got phone calls at home. It came to the point that I didn't enjoy going to work every day. You have to do what works for you. I made money working on the floor, but there are more things." One faculty member responded to the question by saying, "It's all about the students." Two faculty members addressed the question by saying, "I knew I always wanted to continue my education but I could not do 
so in my former clinical position. Education and those considerations will always be a part of me." Another stating, "I guess it would be pretty selfish but you need to think about retirement benefits of which I did not have with my clinical position."

Other themes were prevalent. One faculty wanted to be the patient advocate, another liked hands-on care, and one cited her desire to teach because she had instructors who were very positive. Strongly articulated was the main idea: teaching the students. Still others identified personal concerns in relation to the job. Finally, the big picture issues also received some attention. A nursing faculty member from the "Medium/Large" college group stated, "I got tired in a clinical setting of seeing bad practice, and looking at higher infection rates. We as nurses have to look at changes in Medicare, cost containment, etc."

IS25. Which of these would be the most important factor in your decision to make the change? Why?

Nursing faculty across the groups agreed that flexibility is one of the most important factors in changing careers. Interaction with students, the collegiality, the climate of learning, the rewards of teaching, and the retirement benefits were also included by the subjects as important factors in making the decision to change careers to teaching. One faculty member added, "I hope I can change the way people view nursing. I want to change the way we do nursing care, and not take shortcuts." Another participant added, "The interaction, the classroom, the didactic part, I thought it made me a better nurse. I get rejuvenated in clinical."

Another concern was personal goals. "Retirement was a big factor. I did not have retirement in the clinical setting. With my husband's health [and then death], being with my girls was important, and being able to provide for them." Personal growth also 
surfaced, "I would say it would be the professional desire to advance not only knowledge but also skill."

Educational Attainment by most important factor in decision. The faculty of the groups, "Less than a Masters" and Working on a Master's", chose flexibility, rewards and relationships of teaching. One faculty member added, "I hope I can change the way people view nursing. I want to change the way we do nursing care, and not take shortcuts."

One "Master's" faculty in this group added, "The interaction, the classroom, the didactic part, I thought it made me a better nurse. I get rejuvenated in clinical."

The faculty members "Working on a Doctorate," included these responses to this question. "Retirement was a big factor. I did not have retirement in the clinical setting. With my husband's health and then death, being with my girls was important, and being able to provide for them."

Faculty in the "Doctorate" group included additional responses. "I would say it would be the professional desire to advance not only knowledge but also skill. That is only refined through experience." Also, "I really like the relationships with the students. Students have changed and they are no longer sensitive to others. They also lack a sense of professionalism."

Years of Experience by most important factor in decision. No difference in comparison beyond what was previously discussed.

Size of College by most important factor in decision. No difference in comparison beyond what was previously discussed.

Summarization of Research Question \#4

Nurse educators in this study were asked how they felt when they taught. Words 
empowered, excited, it's almost a high, warm and fuzzy have been used to describe the emotions of nursing faculty when teaching students. The rewards of teaching include the gratification of student performance, the actual presence with the students when the "light bulb goes on," those "aha" moments. The faculty members told of their struggles with maintaining the right relationship with the students; the professional relationship versus the friend. Faculty members verbalized pride when students demonstrate growth and learning from the beginning semester to the last. Nursing educators focus on the immediacy of the classroom setting and the learning process in lieu of the loftier, more distant, and more abstract goals of resolving the nursing shortage.

\section{Research Question 5}

What are the expectations for "being nursing faculty" when first hired regarding:

5.a. Teaching load?

IS26. Think back to the first days of your teaching job. What were the expectations with respect to teaching load? Did this influence your decision to enter higher education? In what ways?

Across the groups the teaching load experience was considered to be overwhelming. Assistance for first semester faculty came in the form of a reduced teaching load or assignment. For other faculty members when a reduced load was not possible, the opportunity to team teach with an experienced faculty member can make the transition easier. In general, faculty in the different subgroups based on Educational Attainment, Years of Nursing Faculty Experience, and Size of College experienced a diverse assignment of teaching loads when they began their new career.

Educational Attainment by teaching load. The faculty in the groups of "Less than a Master's" and "Working on a Masters" varied in their responses to the question on teaching load. One member had a lighter load for the summer but was assigned a regular 
load in the fall. One person did not know what to expect for the first few days of her contract in the fall, "I sat in my office with nothing to do." Another faculty member did not realize until the next year the amount of work, "They gave me some time and did not give me anything upfront.”

The faculty of the "Master's" group responded to their first experiences in relation to teaching load with a range of feelings. On one end are the nurse educators who did not have a heavy teaching load. "No, I did not have a big load. My Program Coordinator was great." Another faculty member stated, "I did not know I was teaching. They were kind to me on the first teaching job. I needed extra time before I had to lecture. But the teaching load has gotten larger, not because of the information but the time allotted."

Additionally, another faculty member in recounting the first year assignments stated, "You had certain hours for didactic, lab, and clinical. You prepared for lecture and I did not think it was overwhelming, but I was young, too." Two other faculty members experienced heavy teaching loads. "My teaching load was a lot. I was overwhelmed. When I first started I had 24 hours of lecture and had to do it right away. I felt like I was catching up, not being prepared."

The faculty in the group of "Working on a Doctorate" also experienced either a heavy load or a very light load. One faculty member recalled, "I did not have any idea what teaching load was. I was team teaching with an experienced faculty member. That was important to me. I think that is important for all new faculty members." Another faculty member also team-taught a course. "It was a gradual process, I had a mentor, and lecture notes from another faculty person." A faculty member who experienced a heavier load than had been expected, recounted her story, "It was a struggle at the beginning. There was nothing [notes] for me to use a couple of times. I wondered if I had made the 
right decision."

All four of the faculty members from the "Doctorate" group experienced difficulty in their transition to teaching early in their careers. "I had no clue what a teaching load was." Another faculty member stated, "I really did not understand anything about teaching. They just said you teach this class and do clinical. It did influence my decision to go back to school." Another faculty member told the story, "Whenever, I first started teaching you took full ownership of the course. You taught the course and clinical by yourself. I think I learned quicker the faculty responsibilities."

Years of Experience by teaching load. No differences in comparison to what was previously discussed.

Size of College by teaching load. No differences in comparison to what was previously discussed.

\section{5.b. Service?}

IS27. In relation to internal and external service to the college, how did your expectations and those of your supervisor differ? How were they similar?

Internal and external service is a part of the responsibility of both community college and 4-year college faculty members. As part of orientation, new faculty receive the guidelines for these expectations and its importance to performance review. For some colleges, internal service is assigned and apparently faculty have little option as to choice. External service to the community according to the faculty in this study is an option for the faculty member to choose. Some faculty in the study felt that the expectation was appropriate, others did not. A small number stated that the expectation as a faculty member for internal and external service was a burden to them. When responses to internal and external service were crossed with the primary categories--Years of Nursing 
Faculty Experience and Size of College--there were no differences. Therefore, these are not included in the discussion of results.

Educational Attainment by service expectation. Of the three faculty grouped into the "Less than a Master's" and "Working on a Masters" categories, two had some orientation to the required activities and one was not sure what it was. "When I first started I just barely knew what external/internal service was. But the Coordinator has been helpful to me in assisting with the planning."

The "Master's" group had seven faculty with one member in her first year of teaching. She had an orientation class and although she was not familiar with internal/external service she received a good explanation. One faculty member related that the demands for external service were a burden for her. Another faculty member voiced a concern, "I feel that most of my time my supervisor agrees with me but it takes time away from teaching."

The group, "Working on a Doctorate" had five members. One did not consider the responsibilities of a faculty role. Another voiced concern, "Internal service makes sense, but no other job tells you to do community service." Still, another had been in a clinical position and had not had the external service responsibilities in that position.

The "Doctorate" group included four faculty. Two had similar expectations as the supervisor. One was concerned, "Internal and external service was not explained to me. When you are new you do not have time; the learning and culture curves are extraordinary." The fourth member accepted responsibility as part of the job requirement. Years of Experience by service expectation. No differences in comparison beyond what was previously discussed.

Size of College by service expectation. No differences in comparison beyond what 
was previously discussed.

5.c. Scholarly writing and research?

IS28. Early in your career, what were the expectations of scholarly writing and research? How did that differ from those of your supervisor?

There were 19 nursing faculty members in this research study. Three faculty members were from programs at the 4-year universities, the other 16 came from the KCTCS Community College System. Responsibilities for research and scholarly writing are not assigned to faculty members in the Community College System. The three faculty from 4-year colleges are strongly encouraged to participate.

5.d. Earning tenure and/or promotion?

IS29. How much of a concern were the requirements for earning tenure and promotion when you decided to become a faculty member? In what ways?

Educational Attainment by promotion and tenure. The three faculty members in the "Less than a Master's" and "Working on a Master's" groups are not assigned a tenure track position. However, they are on a promotion track. One faculty member stated, "I am not really concerned about tenure and keeping my job. I want to satisfy myself; I am my hardest critic." Another new faculty member was concerned about not being on a tenure track, "It kind of bothered me to not have the opportunity for it but in the clinical setting your behavior can cost you your job." The third faculty member stated she asked at the interview about promotion and tenure. After she was hired, "I was told there was a dispute and I am not on a tenure track now, but a promotion track. I am sure it would not have made a difference, but they didn't say that at hiring."

The "Master's" group is composed of seven faculty of which one had been employed for two months in a full-time position, three faculty had been at their respective colleges for at least six years, and three that had been at their respective colleges for at 
least 11 years. One who had been there for at least six years was surprised at the difficulty in locating important documents that go into the promotion file. "I didn't realize I should have been collecting that information and pulling it together. It was hard to put everything together in a short period of time. When I went up for associate it was a little easier." Another faculty member stated," First we had tenure, now we don't. But we still have promotions. It may mean more to the older faculty. If I wasn't doing a good job I should leave." The newest faculty member in the group discussed her understanding of tenure. "They talked about tenure to us in orientation and it is a question mark in my mind. I understand if you don't get tenure, you lose your job. So at this point it doesn't have a positive connotation. I will work to increase my skills."

The group "Working on a Doctorate" had five members. Two have been teaching from $0-5$ years, two are in the 6-10 year group, and one has $11+$ years of experience. The faculty member with $11+$ years in teaching stated, "I think it was drilled in me how important it was with percentages and keeping good files. My mentor advised me and I have mentored others." Another faculty member with 0-5 years of experience stated, "I did not have any knowledge of a promotion process. But I had a really good mentor. Documentation was important. I also learned tenure had a negative connotation. But it should be a safeguard to be able to ask ineffective faculty to leave.

In the "Doctorate" group there is one faculty person with less than five years of experience, one from 6-10 years, and two with $11+$ years of experience. One faculty member with $11+$ years of experience stated," It wasn't even on the radar frame. At the time if you did an OK job you got tenure. Tenure is going away."

Years of Experience by promotion and tenure. In the "0-5" year group, faculty are learning about promotion and/or tenure within their college settings. For some programs 
within the community and technical college system, tenure is no longer an option, as some of the faculty have reported in their interviews. One faculty member in this group said, "I think it is stressful. You only have five years to work on all these things. The university focuses on teaching and it is difficult to do all of those other things." Another faculty member came into the system with teaching experience. "I didn't know what it was, I received two years teaching experience and it could be harmful for me. If I don't get it I am gone."

Tenure and promotion from the " $6-10$ " year group included those who had at least completed the process for the first step of promotion. One faculty member stated, "I did not know anything about tenure, but it would not make me stay or go. It could be a perk." Another faculty member who was in a tenure track within the community college system noted, "My first role was not a tenure track position but present is. I looked positively on tenure. It provides freedom to speak up for students and peers. It also adds to the prestige of the educator role and distinguishes you from the non-experienced faculty."

In the "11+" faculty group many may have already been promoted to professor. From a 4-year faculty member in the group, "I didn't understand the whole thing at my previous college. But I am not eligible for tenure here. I don't really care because at 65 , I will be gone." Another member of the group stated, "That was just a headache more than anything; jumping through the hoops. It was not a benefit for me."

Size of College by promotion and tenure. No differences in comparison beyond what was previously discussed.

\section{Research Question 5 Summarized}

This chapter dealt with the expectations for "being nursing faculty" when first hired. The faculty members shared their concerns and their experiences as they 
progressed through the social institutions of academe. Most of the faculty were supported and mentored as they began their first days as nurse educators. Some told stories of their thoughts and feelings those first few days of teaching.

Several faculty had a lightened teaching load their first semester and were assigned a full load the next semester or next year. Many were assigned a full teaching load with the assistance of an experienced faculty member. For a few study participants, a full teaching load with no assistance brought responses of being overwhelmed yet they persevered.

The faculty responsibilities of internal and external community service, from the nursing faculty member's point of view, has been something that is done because it is required, or faculty assign themselves a service project because the expectation for performance review is that it must be done.

Promotion and tenure in some of the colleges has become somewhat controversial. New faculty seem to be unaware of the meaning of promotion and tenure, much less the process. Of all the comments from these ADN faculty (primarily community college), there was only one that encapsulated the traditional meaning and concerns of tenure in higher education i.e., freedom to speak up for faculty and students without political repercussions. This suggests that tenure has a different meaning at this level (community college, 2-yr degrees) than in traditional higher education (4-year institutions especially those with graduate programs). From the interview questions, the thoughts and feelings of this group of faculty have provided insight into the faculty processes and the organizational culture in their colleges.

\section{Research Question 6}

What are the primary barriers that you experienced in becoming a nurse educator? 
6.a. Student evaluations?

IS30. Thinking back to your early days as a nurse educator, relate your knowledge of student evaluation. How confident were you? Did this enter into your decision to enter teaching?

The interview responses from the nursing faculty revealed that they were not confident with student evaluations. The process of small groups assessing faculty performance can be devastating, even if only from one or two students' negative judgments. Despite these negative sentiments, student evaluation was not an important factor in entering teaching, based upon these discussions, perhaps because new faculty from a clinical background would be unfamiliar with the process.

Educational Attainment by student evaluation. The "Less than a Master's" and "Working on a Master's" groups had three faculty members with a total of four years teaching experience. Each verbalized a feeling of anxiety about student evaluations. In observing body language as the question was asked by the researcher, usually the first words were followed by a deep sigh. One of the faculty members stated, "I thought I had done the best job I could do." One faculty member added, "It is amazing when these people are in the same classroom and have two different views. Some days I guess I intimidate them and some days I have Ann Landers written on my forehead." Another faculty member, "I was giving $110 \%$, they should too." This did not enter into any of this person's decision to enter teaching.

Of the seven faculty from the "Master's" group, one subject verbalized a lack of confidence. "Students evaluate more on personality than what you do in the classroom. If they make a bad grade they judge you." Another faculty member noted, "Evaluations are held over our heads. Small groups can really be a problem. They are not evaluating me on 
whether I can teach, but how they did in the course. They can be a problem for someone going up for promotion or tenure." Taking the criticism and moving forward can be a challenge for faculty. "Sometimes you can agree and disagree with student evaluations. Some hard evaluations can cut you to the quick. You pick yourself up and you change what needs to be changed and you go on."

In the "Working on a Doctorate" group the five faculty all shared a lack of confidence with student evaluations. "Another shocker, you perceive yourself one way and the students see you another. I don't like waiting until the end of the year to find this out." The impact on the evaluation can be overwhelming. "I was surprised when I came up for evaluation how much they impacted your rating. Bad comments even when the majority are good are mentioned in your evaluation. In the clinical setting my job evaluation was based on my performance-not patient satisfaction."

In the "Doctorate" group three of the four faculty were not confident with student evaluations. "I was not confident and I did go back to school to get a Master's with an emphasis in teaching." Another faculty person responded to the question in this manner. "I don't believe in student evaluations. You are always going to have personality conflicts. If you have eight people and one person hates your guts--you are screwed."

Of note, the interview results show that the faculty misunderstood the question. Yet this paragraph, while accurately summarizing the interviews, is extremely misleading regarding the content of IS30. This question was intended to seek responses related to the skills of novice faculty in evaluating their students. This is (was intended as) one of several pedagogical issues addressed in RQ6. The nurse educators viewed this as being evaluated by students. That this was consistently misinterpreted, across all groups by all levels, indicates how salient, how powerful is the process of student ratings of faculty, 
presumably because of the emphasis in these programs on teaching excellence and use by administration of these data in faculty evaluations (and eventually promotion and tenure). When the responses were compared across the variables, Years of Experience and Size of College, the experiences were similar and no additional information was identified.

Years of Experience by student evaluation. No differences in comparison beyond what was previously discussed.

Size of College by student evaluation. No differences in comparison beyond what was previously discussed.

6.b. Clinical evaluation of students?

IS31. In the early days as a nurse educator, relate your knowledge of clinical evaluation of students. How confident were you? Did this enter into your decision to enter teaching?

The faculty members were the most comfortable in the clinical setting and felt somewhat confident in evaluating the student in the performance of skills. Several of the faculty members elected to use their previous nursing unit to take students for clinical experiences. Some faculty voiced some reservations about assessing the student using an evaluation form of untested validity. In general, the ease and sense of confidence appeared across all 19 of the interviews. Typical were comments by one member who said, "I felt more confident because I was very strong clinically and I wanted to help strengthen their skills." Another said, "I was very confident in the clinical setting, it was my area of expertise." These sentiments were so common that lack of confidence stood out, as voiced by this participant, "I really did not know how to evaluate students clinically other than give feedback. I wasn't as sure I was doing it correctly, but I tried to be honest with the students." These sentiments as expressed are captured across the subquestions for Educational Attainment, Years of Experience, and Size of College. The 
experiences in the latter two were very similar and no new information was indentified.

Educational Attainment by clinical evaluations. The "Less than a Master's" and the "Working on a Master's" groups were made up of three faculty with a combined total of four years of teaching experience. All three were confident in their clinical skills. One faculty member related, "I feel more comfortable in the clinical setting than in the classroom. I am located where I formally worked. Sometimes I have to put my hands behind my back to keep from helping out on the floor."

All seven faculty members in the "Master's" group expressed their comfort level in evaluating students in clinical. One faculty member discussed her comfort level with her skills, but was concerned about being able to convey her message to the students. "I was confident in my clinical abilities, but I was not sure I could get that across to students. I think doing clinical on the same floor where I previously worked was a great support for me." Another faculty member who was in her first year of teaching felt comfortable with her skills, but she saw a problem in evaluating them. "I am strongest in clinical evaluation because my expertise is clinical. To better evaluate the students, I want to develop more assertiveness in my approach to them."

In the "Working on a Doctorate" group, all five of the faculty members stated that they were comfortable taking students to clinical and evaluating them. One faculty member was quite confident in her skills, "I was comfortable with the clinical side, but the didactic took me a while to get comfortable. I have a fear of not being able answer their questions."

In the "Doctorate" group faculty reported that they were confident in clinical. One faculty member suggested that there needed to be more education on clinical evaluations, especially the cultural backgrounds of the client. 
Years of Experience by clinical evaluations. No differences in comparison beyond what was previously discussed.

Size of College by clinical evaluations. No differences in comparison beyond what was previously discussed.

6.c. Preparation of lecture content?

IS32. In the early days relate your knowledge of preparation of lecture content. How confident were you?

The faculty members across all groups reported that they were not confident in the preparation of lecture content. Only two of the 19 faculty reported some level of confidence. Typical responses to the question were: "I tried to include too much on a PowerPoint slide. Now I am backing down. I want to focus in on just the little bit that will give them the overall picture. I tried to put everything in the book; I just tried, and if that doesn't work, change it up." One suggested perseverance, "I was weak, not good at all. I didn't have a mentor assigned. The more you teach, the better off it gets." Across the interviews, the different subgroups varied little as reflected for Educational Attainment.

Educational Attainment by lecture content. Faculty in the "Less than a Master's" and "Working on a "Master's" groups responded that they were not comfortable with preparing lectures. One faculty member discussed the time element of preparing a lecture. "That was scary initially. The textbook had great resources and an instructor was there to help. I prepared for a two hour lecture and it lasted 20 minutes." Another member from the group evaluated her progress. "It is getting better with each one. I anticipate questions, and after I finish, I go over how I presented it. I always ask myself, could I have done a better job?" 
Faculty from the "Master's" group shared their concerns regarding lecture preparation. "I wasn't very confident at the time. I think I gained knowledge when I looked at the NCLEX test plan and the RN practice analysis. It helps new faculty prepare lectures." Another faculty member said of her comfort level, "I had no confidence whatsoever."

In the "Working on a Doctorate" group one faculty member reported that her Master's program had prepared her well. "I had to start from scratch. My mentor helped me." Another faculty member related her experience, "I was not very confident at all. That was just awful. The team teacher helped me; I was more technology oriented and she helped me with the content."

The "Doctorate" group had a faculty member who felt somewhat confident in lecturing. "I started out somewhat confident in preparing lectures and had good mentors to guide me in preparation and delivery. Another noted, "I remember my first lecture. It was scheduled for two hours, I was done in 30 minutes. Now the problem is the opposite-I have too much I want to tell them."

Years of Experience by lecture content. No differences in comparison beyond what was previously discussed.

Size of College by lecture content. No differences in comparison beyond what was previously discussed.

\section{6.d. Examination development?}

IS33. In the early days of your teaching position, relate your knowledge of development of examinations. How confident were you?

Most faculty members in the study reported that they had little knowledge of the development of examinations. One faculty member was "pretty confident" with the use 
of test banks and other material. Several faculty members reported that despite teaching for several years, the preparation of test questions is an ongoing process. Several members reported early in their career they were able to attend workshops dealing with test development.

Educational Attainment by preparation of examinations. In the "Less than a Master's" and "Working on a Master's" groups, the three faculty had all attended workshops to assist in the development of tests. One faculty member prepares the questions prior to lecturing on the content being introduced in lecture. "It works out very well when I actually can have the test developed before the lecture and I actually talked about the content."

In the "Master's" group, one faculty member from the seven provided this information, "It was a work in progress. Each year test questions are tweaked. It is a good feeling to have good test questions. I always work on new test questions; that is a skill in itself."

In the "Working on a Doctorate" group, the five faculty members reported that none of them were confident. Some of the faculty members relied on test bank questions and although they were not pleased with the outcome, it provided a point to begin. One faculty member related, "I knew nothing about examinations, but I was able to go to a conference my first semester. I felt better about myself and my abilities. I think it is an ongoing process you have to keep perfecting."

In the "Doctorate" group, one of the faculty members reported, "My tests were so easy a first grader could answer, or so esoteric it was like a curvy road to get to the answer. If the lower students did well it was a good test."

Years of Experience by preparation of examinations. In the " $0-5$ " group the 
faculty who were not confident in the test preparation initially used test banks. Three of the seven reported they had attended workshops to assist in the process. One faculty member described a pool of test questions used by the faculty from which she tries to select the best questions, "I struggle with the students who just want to know what material is going to be on the test, so preparation will only come from that material." One faculty member became an item-writer for a company.

Of the seven faculty in the "6-10" group, some attended conferences to assist in the process, some used test banks as a beginning, and all agree that it continues to be a work in progress.

Of the seven faculty members in the " $11+$ " group all reported that it is a difficult task for all faculty members. All seven of the members struggled in the beginning to develop appropriate questions to measure the knowledge of the students. All have used test banks as a beginning, and all agree that they continue to hone their skills in this area.

Size of College by preparation of examinations. No differences in comparison beyond what was previously discussed.

6.e. Curriculum revision and design?

IS34. In the early days of your teaching position, relate your knowledge of curriculum revision and design. How confident were you?

Of the faculty members in this study, very few have had the opportunity to be part of the curriculum review process. Certainly it does not occur on a frequent basis as evidenced by the number of people who have not been involved. Of the study members, five had been on curriculum revision committees (one had chaired a committee), four had course work in their Master's program, and ten had not been a part of the process. Representative thoughts are presented by the various subgroups of faculty members on 
the Educational Attainment, Years of Experience, and Size of College subcategories. These were essentially the same across the board.

Educational Attainment by curriculum revision and design. Of the three "Less than a Master's" and "Working on a Master's" faculty members, one had not been exposed to any of the process, one attended a conference and became familiar with the terminology, and one was placed on a 20 -member committee where revision of the curriculum was in progress. The faculty member who was part of the committee stated, "With 20 other people on the committee it was helpful for me to see why we are teaching that content."

The "Master's" group had seven faculty of whom two had taken curriculum design courses in their advanced degree programs, two were involved early in their teaching careers when their programs were undergoing a curriculum revision, one had served on a curriculum committee and had chaired it, and two were not part of the process.

The "Working on a Doctorate" group had five faculty members, two having had curriculum design coursework in their Master's program, two had served on committees and one has been part of a minor revision of the program's curriculum.

The "Doctorate" group had four faculty members, one member had served on a curriculum revision committee, the other three have little experience with the process.

Year of Experience by curriculum revision and design. No differences in comparison beyond what was previously discussed.

Size of College by curriculum revision and design. No differences in comparison beyond what was previously discussed.

6.f. Technology? 
IIS35. In the early days of your teaching position, relate your ability in the use of technology in the educational setting. How comfortable were you? How did that differ from your use of technology in the clinical setting?

There were 15 faculty who reported that they were comfortable with technology. There were four who were not. Many of the faculty members who indicated they were comfortable are using advanced technology in their courses on a daily basis. Examples include ITV, podcasts, clickers, iPods used in clinical, Blackboard, and patient simulation. During the interview sessions several faculty voiced a concern for the overuse of PowerPoint and the unintended change from students failing to read the textbook and only using the handouts of the slides. Those concerned faculty have been exploring alternative options to the trend. Some of the faculty reported that their previous comfort level with technology in the clinical setting made the transition successful in academe. Several faculty commented there is more technology in the educational setting than in the clinical setting. The comments from faculty in subgroups of Educational Attainment are representative of the subgroups for Years of Experience, and Size of College.

Educational Attainment by technology use. All three faculty in the "Less than a Master's" and "Working on a Master's" groups are comfortable with technology and use it for lecture and in clinical.

Three of the seven people in the "Master's" group indicated they are not comfortable with technology with four being comfortable, using it in a variety of ways. One faculty member who is not comfortable with technology stated that it was one of her weaknesses. "It's not that I don't want to learn, but I am a generation removed from students. There is a co-worker hired at the same time and she is very technology literate, she helps me with coursework. I was given an iPod and I am trying to learn. There is 
more technology in education than clinical setting."

All five faculty within the "Working on a Doctorate" group are comfortable with technology. One of the faculty who was very comfortable with computers said, "I have always loved computers. I was the first in the department to use PowerPoint podcasting. I have been doing presentations nationally on podcasting."

The "Doctorate" group of four faculty has three of them are very comfortable with technology and one who is not. One member if this group is comfortable with technology and stated," I am pretty progressive with technology. I believe it depends how the brain processes. I taught in a college that had 'dummy terminal' Then today I used clickers for the first time."

Years of Experience by technology use. No differences in comparison beyond what has been previously discussed.

Size of College by technology use. No differences in comparison beyond what has been previously discussed.

IS36. Compare your ability to organize and manage your time in your teaching position with your position in the clinical are. How does it differ?

Faculty across the groups were divided on their responses to the question. Six faculty responded that the clinical setting required the most organization and the management of time was most crucial. Eight responded that teaching required the most organization and time management was the most difficult. Three noted that both areas require a great deal of organization, and one faculty member responded that it was difficult to compare the two since the areas were totally different. The subcategories captured under Educational Attainment seems to match the subcategories for Years Experience, and Size of College. Representative of the frustrations necessitated by the 
higher education milieu, in general, was this comment from a faculty member with " $11+$ " years of experience, "Clinical is more organized. There is difficulty in planning time for teaching. It is frustrating because organization had never been a problem before."

Educational Attainment by organizational issues. Of the three faculty members in the "Less than a Master's" and "Working on a Master's" groups, two related that there was more control of time in teaching. The unpredictability of clinical was cited as the reason for the belief. The other faculty member stated that there was more structured time in the clinical setting. She found that managing her time was difficult. "Students and faculty are in and out and the day never goes as planned."

The "Master's" group with seven faculty; four faculty members identified the teaching setting as being where the most organization was a necessity. Three felt it was the clinical setting that was the most organized. One faculty member cited the time necessary to prepare for clinical or classroom. "I did not have to prepare for clinical. There was time spent in preparation for classroom." Another faculty member cited the necessity for organization in the classroom. Students come from distances and keeping the class on time is essential.

The "Working on a Doctorate" group has five faculty members. One faculty member identified that the college required more meetings on and off campus. With clinical, all activities would be on site. Another member saw clinical as requiring more organization due to working committee meetings around patient care. Two faculty noted that both clinical and college required organization.

The "Doctorate" group has four faculty members and each had a different opinion on organization and management of clinical as opposed to the college setting. One faculty member identified clinical as being the most rigorous and teaching required the most 
organization of time. Another identified the clinical setting as the place where it is easier to manage time. One stated that clinical is the easiest to organize time and manage.

Years of Experience by organizational issues. No differences beyond what was previously discussed.

Size of College by organizational issues. No differences beyond what was previously discussed.

IS37. What is your sense of the collective cost/benefit of these barriers compared to clinical nursing? Has that influenced your decision to remain a nurse educator?

Each of the faculty members discussed the issue of salary and benefits and the continuing salary disparity in relation to the clinical setting. Many have said that if they wanted a higher salary they would have left education and returned to the healthcare setting. For many of them, the rewards are not monetary, but rather the feeling of pride when a student succeeds or graduates to become a much needed, caring nurse. In general these nursing faculty members weighed other benefits against the cost of salary. For example, two of the members were employed part-time prior to becoming full-time so they did not take the pay cut that someone from a full-time clinical position would take coming into a teaching position. Consistently, faculty across the various groups mentioned salary shortcomings. In contrast, beyond the love of teaching and pride in their students' success, the benefits to remaining a nurse educator included the flexibility of schedule and the value of technology. Despite this, these nursing faculty would like to be paid a salary close to that of a clinical person with the same education and years of nursing experience.

Educational Attainment by cost/benefits compared to clinical. There were three faculty who made up the "Less than a Master's" and "Working on a Master's" groups. 
All three faculty discussed the benefits surrounding salary for their decision to become or remain a nurse educator. One faculty member responded that, "Benefits, tuition for MSN, salary opposed to clinical setting; it's disheartening to know that the LPN sitting next to you is making what you are. But you are off in summer and they are not."

The "Master's" group had seven faculty. One faculty member relates, "I am not in teaching for the money. I enjoy it or I would return to clinical if I did not enjoy it. Teaching has become a part of me. I enjoy what you get from teaching and watching students grow. I would have palpitations if I had to leave nursing."

The "Working on a Doctorate" group had five faculty. All identified the benefits as opposed to the salary for staying in education. One faculty member noted, "On the salary side I took a big pay cut, but retirement is a plus. I am glad I have a job with the present economy. I won't quit because of lack of money."

The "Doctorate" group had four faculty. Two faculty members considered the benefits over the salary. One member from the "doctorate group bargained for more money at her college and was rewarded with an increase. The remaining educator examined the technology barriers and made the decision to stay based upon the availability of technology.

Years of experience by cost/benefits compared to clinical. No differences beyond what was previously discussed.

Size of college by cost/benefits compared to clinical. No differences beyond what was previously discussed.

\section{Research Question 6 Summarized}

This research question addressed the primary barriers that nurse educators experienced in the beginning of their career. Each of the different responsibilities is 
addressed in relation to the confidence level of tackling that responsibility. For this group of nurse educators, the tasks they were most comfortable with were clinical evaluation, technology, and organization and time management. For the new faculty members in the 0-5 years of experience category, the overwhelming nature of tackling these responsibilities surfaced. Each of the new faculty found or were assigned mentors or cofaculty to assist them, work with them, and ease the transition. The nurse educators in the mid-years of their careers (6-10 years of experience) reflected on the value that they bring everyday to students who are part of their programs.

Salary and benefits brought much discussion. Each faculty member realized that salary was not appropriate for the position. Each realized there were many benefits which were attractive enough to outweigh the financial rewards.

\section{Research Question 7}

IS38. How satisfied are you with your job and performance? Why and in what ways?

In this study, nursing faculty were asked about their satisfaction, or lack thereof, about their job performance. Nursing faculty members were very candid in their selfevaluations. A large percentage of them are very satisfied with their job performance. One faculty member seemed to always look for self-improvement and one faculty member is contemplating leaving her present employer for a variety of reasons, but had not received positive feedback for her achievements.

Representative of comments across the 19 nurse educators, reflecting their general satisfaction with their job, were the following. One with little experience responded, "I am very satisfied with my job. I have improved in my teaching. This is where I want to stay." Others with mid-year experience had similar feelings. "I am very satisfied with my 
job performance. I contribute much to the program by being a conscientious and hard worker." Another commented about herself, "I am pretty satisfied. I am pretty comfortable in what I am doing. I feel I am able to judge the students and help them achieve what they need." Those with the most experience responded in kind, with one adding, "I like my job and I think I have performed well. My community service needs some improvement. I can get work done." Such sentiments were common for different levels of educational degrees as well as levels of experience.

Educational Attainment by job and performance. All three faculty of the "Less than a Master's" and "Working on a Master's" group were satisfied with their job performance. One faculty member noted, "I am satisfied with my job and responsibilities. I am very content. I am still learning and changing." Another member of the group states, "I like teaching and taking students to clinical. Hopefully, my job performance is up to the standards of the college."

Six faculty members of the "Master's group rated their job performance between satisfied to very satisfied. A member of the group added, "I am very satisfied with my job. I have always enjoyed students. I love the eagerness of learning and excitement in the beginning students. I am always tweaking my lectures and I feel like there is always room for improvement." A new faculty member in this group responded, "I think I am doing satisfactorily in both the classroom and clinical. I will feel better when I am more comfortable. I think I am performing satisfactorily for a novice educator." The only member who did not rate herself as satisfactory was not satisfied with her job in general terms. "I think that there is always something that one can change and do."

Three members of the "Working on a Doctorate" group replied that they were very satisfied with their performance. One of the members added, "I am very satisfied 
with my job performance, I believe that I contribute much to the program. I am conscientious and a hard worker." One of the faculty members had been re-assigned for the current semester to develop an on-line nursing program. She rated her job satisfaction as "OK." The last member of the group replied that, "This is a low point of the job. The faculty has decreased in size, we are doing more, administration has changed, and despite two national presentations it was not reflected on my annual evaluation. I am looking for something else."

The four members of the "Doctorate" group responded positively about their performance. One member added," I am very satisfied with my job. I would not have put in all the years if I didn't. I am happy with my performance and my student evaluations are exemplary." Another member responded, "I am very satisfied with my job as an educator. I am a mentor to new faculty, I have an ability to multi-task. In this job I am able to continue my education and maintain my professional and personal endeavors."

IS39. In the last year how many times have you received feedback for your performance? In what areas and why? To what extent is this influential in your career as a faculty member?

The nursing faculty subjects received frequent feedback from a variety of individuals. The Program Coordinator was a key person in providing feedback as well as fellow faculty, Division Chair, and the students.

Educational Attainment by feedback received. The faculty in the "Less than a Master's" and "Working on a Master's" groups included three members who have received feedback from instructors, Program Coordinator, mentors, and students. One new faculty member added, "I actually feel fortunate here, I have received positive feedback from my Program Coordinator, a fellow faculty member came in and listened to my lecture. I would say that I have received positive feedback about my performance 
many times."

The faculty in the "Master's" group included seven faculty members. In total, feedback toward the faculty came from students, fellow faculty, Program Coordinator, Division Chair, President, and Academic Dean. A nursing faculty member stated, "We get feedback every day from students. Don't care about anyone else. The student feedback is the main thing that keeps me in teaching."

The faculty in the "Working on a Doctorate" group included five members. One faculty member stated, "The last three years--excellent performance evaluations. Program Coordinator gives excellent feedback. Nursing went under the microscope, an external reviewer. Why are too many not making it retention. How do you balance that? I want us to turn out nurses that the facilities will value."

The faculty in the "Doctorate" group included four members. All reported positive feedback from many sources, including the Division Chair, Program Coordinator, and students. A faculty member stated, "I have received positive feedback for excellent clinical and academic advising, integration of technology, and program accreditation standards. This is very motivating."

Years of Experience by feedback received. The " $0-5$ " group had seven faculty members who received positive feedback from a variety of people. A faculty member discussed who gave them feedback, "Feedback came from faculty, Chair, and faculty members. The Coordinator comes to lectures four times in a year." The clinical staffs offer positive feedback to faculty and to students.

The "6-10" group had five faculty. Feedback came from Program Coordinator, faculty chair, and administration. One faculty member had not received any positive feedback, and received negative feedback from Division Chair. She had given much to 
the program but does not feel appreciated. Another faculty member explained, "The first couple of years I received very little feedback, now I receive more. I appreciate criticism, good or bad. We need to understand where we can improve."

In the "11+" group there were seven faculty. Six faculty received positive feedback and one has not received positive feedback. One faculty member stated, "I have received feedback from the Chair. She is bringing our school back into the 21 st century. Getting positive feedback is probably more than money can buy."

Size of College by feedback received. No differences in comparison beyond what was previously discussed.

IS40. Who gave you that feedback? In what areas and why? Does the person who is giving positive feedback influence your satisfaction as a nursing faculty member? In what way?

Feedback to the research subjects comes consistently from the Program Coordinator and fellow faculty members. Student feedback is also important to these nursing faculty members. Typical of perceptions were comments such as these. One shared the nature of the feedback, "The Program Chair has been very helpful. She had come to my class and gives me feedback which is the latest methods. She had helped me grow professionally, and she is one of my biggest fans." Another noted, "The Coordinator and co-faculty as well give support. The whole atmosphere is great." Several also indicated the feedback from their students as being positive, "The students influence my satisfaction as a faculty member."

Educational Attainment by source of feedback. The "Less than a Master's" and "Working on a Masters" group consisted of three faculty. A faculty member from this group stated, "Feedback is very influential. If I never received positive feedback I would think I was not doing a good job and I would leave." Another group member explained 
the relationship the nursing faculty have at her college. "We are a close-knit group and help and support one another. When I am having a bad day someone will anonymously leave a chocolate piece of candy on my desk."

The "Master's" group had seven faculty. The entire faculty reported they had received feedback from the Program Coordinator, fellow faculty members, and students. One faculty member related, "The Program Coordinator gives me feedback. She is guiding and answering my questions. I feel that the faculty are always ready to help me. It makes me satisfied in my job and that my ideas are validated. I want to do the best job of my ability."

The "Master's" group has seven faculty. One faculty member discussed the role of the Program Coordinator in support of the faculty. "The Program Coordinator does the little things to show appreciation. I get more satisfaction when they tell me they like what I do." Another faculty member discusses the importance of student feedback, "Feedback means more when it comes from students. They are who we are interacting with the greatest majority of time. When the exchange of knowledge has occurred they are getting it."

The "Working on a Doctorate" group has five members. All provided examples of their sources of feedback and the impact as a result. One faculty member describes the source of the feedback and the impact. "Faculty members, the Coordinator, Division Chair, and students give feedback and provide satisfaction of the nursing faculty role. I don't need constant feedback to feel worthy of being here. I get the satisfaction from just being with my students. It is internal.”

The "Doctorate" group has four members. One member of the group describes the important impact of feedback of colleagues. "Colleagues and community members give 
feedback to me for my knowledge and contributions which has contributed to the satisfaction of me as a faculty member."

IS41. What is the nature of the positive feedback you receive? Formal recognition? Informal encouragement/praise? Concrete (as in course release, raise, other)? Psychic (as in good for the self and soul)?

Faculty members in the study have received very little formal or concrete feedback. The informal feedback seems to provide immense satisfaction for these educators. Typical of perceptions of feedback faculty received is this comment, "I would say that it is more informal encouragement and verbal praise. No, not concrete, that has to come. It would be psychic, it mentally makes me feel good." This participant identified formal and psychic satisfaction, "It is a combination of informal encouragement and praise as well as psychic, an overall feeling of wellness."

Educational Attainment by type of feedback. The "Less than a Master's" and "Working on a Master's" groups had three members. One has received more informal recognition. Another has received concrete feedback, and likes to hear "job well-done" which is good for the psyche. Another one of the group noted that she wanted a little bit of both, a raise and a "little piece of chocolate."

The "Master's" group had seven faculty. One faculty member concluded that she had received informal feedback since the Coordinator does not have responsibility for money. The Coordinator tells them she appreciates them quite frequently. The faculty member says, "It is nice to get that feedback."

The "Working on a Doctorate" group had five faculty members. One of the members receives a combination of feedback, "You get that informal feedback by comments that are made. You get that formally in your performance evaluation where we have to do a special project to get the EE rating." She said, "I like to say I like to stir the 
pot a little bit."

The "Doctorate" group had four faculty members. One member noted of the positive feedback, "It has been psychic, since no raise, I don't know that I've gotten any formal recognition for positive feedback."

Years of Experience by type of feedback. No differences in comparison beyond what was previously discussed

Size of College by type of feedback. No differences in comparison beyond what was previously discussed.

IS42. How much do the different types of satisfaction influence your career choice as a nursing educator?

The members discussed the rewards of raises and benefits. However, the informal feedback from key people in the college and students has been very satisfying. Representative of responses across the primary subgroups include the comment from one member, "I have to be happy in doing what I am doing. My mother could not understand why I chose nursing education when she saw the salary. Comparison rewards are the flexibility. Could I go back to the bedside and perform excellent care--absolutely. But I truly believe this is where I am meant to be." Another verified the success of providing feedback, "The satisfaction from the personnel and others was very influential in my career choice." Another response concerning satisfaction, "It's what keeps me here--for a better lack of words." For another member, the source of satisfaction was the dean of the program, "My Dean and her management style. She lets me do the job and then I hand it to her. She doesn't micro manage me. She lets me be free. She gives me the opportunity to be free."

Educational Attainment by type of satisfaction. The "Less than a Master's" and 
"Working on a Master's" group had three members. One replied, "I don't have to constantly hear good things. When I get satisfaction from students and faculty, it makes me strive to do a good job." Another faculty member stated, "I think you have to have all that to stay here."

The "Master's" group had six faculty members. One faculty member stated, "In a clinical position you do not get much feedback from managers in the clinical setting. I feel more valued here. In the other setting I could just go days and just clock in and out with no interaction. Here I have interaction with superiors and co-workers."

The "Working on a Doctorate" group had five members. One member stated, "Getting positive feedback makes me feel I am making contributions to the program. I think if that was negative, you would not want to stay. With my experience it has been very influential in staying here."

From the "Doctorate" group with four members, one said, "I think the psychic, there have been very few raises. The positive feedback from students is a good thing."

Years of Experience by type of satisfaction. No differences in comparison beyond what was previously discussed.

Size of College by type of satisfaction. No differences in comparison beyond what was previously discussed.

IS43. Do you have any final thoughts or feelings about being a nursing educator? To what extent have these influenced your satisfaction with your career choice?

Final thoughts from this group of 19 nurse educators were very similar across the three primary subgroupings for this study: Educational Attainment, Years of Experience, and Size of College. Only Educational Attainment is broken out separately.

Educational Attainment by final thoughts. The "Less than a Master's" and 
"Working on a Master's" group had 3 people in the group. One summarized, "I don't want people to view nursing education in a negative connotation. One has to have their own opinion, but experiencing for oneself what it is we do." Another member said," With most nursing positions you can do new things, learn new things, and it's the same with the nurse educator. I want to be able to continually grow and learn new things and be with students. It has a great satisfaction for a career." The third stated, "It is a rewarding job and in the end on graduation day you may have started out with 40 and end up with 20. It is rewarding to know that you have prepared them. If they wanted to go on, you are a part of that education."

The "Master's" group had six members. One group member stated, "I wish every nurse educator didn't have so many students with misconceptions about what nursing is. I wish we could have nurses paid on par." Another faculty member said, "I figure I will be working another five years and attempt to stay in it." Still another, "Success of graduates is the most important and rewarding. Success stories keep going." Added one member, "I have felt some measure of success and achievement from it. I do think I do okay and do think I have something to offer students. I am tired and want more money for the work I've done."

The "Working on a Doctorate" had five members in the group. The comments made by the members in answering the last sub-question include the following: (a) "I want to ignite that spark for students. I want to be able daily, yearly, moment by moment [to give] my students more," (b) "I just enjoy teaching and I love the combination of teaching and nursing," (c) "I hope this is going to be a long term commitment. I like the community college setting, and you can get involved, it is more like family. I have made a good choice," (d) "I think students should see what goes on behind scenes, we have to 
deliver a good product. What is keeping me here--satisfaction in seeing them graduate and practice safely. Contact with graduates who are successful makes it worthwhile," (e) "I love being a nurse educator," (f) "More about students, I think I get appreciation and a sense of respect," and (g) "I really enjoy being a faculty member, I really feel good about myself and have a sense of accomplishment. I was the first one to graduate from college in my family. I am a good role model for my two girls."

The "Doctorate" group had four members. Responses to the last question from that group include the following: (a) "We need more of us," (b) "Rewarding experiences, many options, roles, administration, etc., for those interested in nursing," (c) "I enjoy what I do. It is a different kind of stress from the acute care setting," and (d) "This is the happiest I have been as a nurse educator. My Dean has influenced my satisfaction," and (e) "Being a nurse educator is a rewarding experience opening a variety of opportunities."

\section{Summarization of Research Question 7}

All nineteen faculty responded to questions dealing with the work environment. The greatest majority of faculty members are pleased with their job and performance. New faculty are struggling to do a satisfactory job and their comments demonstrate that they are pleased with their career selection. For those faculty members in the community college system, raises have not been awarded for the last three years, creating an even greater lack of parity for pay in a field already under-compensated. However, despite the lack of those concrete monetary rewards, the feedback the nurse educators are receiving seems to be sustaining them. They are pleased with their performance, they have been receiving important feedback, and their everyday interaction with students seems to energize them. On the down side, one senior faculty member who is not getting that important positive feedback and support admits that she is at a low point in her career and 
is looking for another opportunity.

\section{Summary}

Each of 19 associate degree nursing faculty members from a purposeful sample completed two instruments; the Background Questionnaire for Nurse Educators, and the Nurse Educator Interview Schedule. The participants shared their experiences and insights from their decision to enter nursing, to how they felt about their job and their role as a faculty member. The rich descriptions of this group of nurse educators provided details of a love of learning, a passion for working with students, and a desire for flexibility. They also were gratified by the feedback from the students as well as their fellow faculty and supervisors. The descriptions of their concerns, their joys, and their rewards with teaching in associate degree nursing programs in Kentucky were enlightening.

The researcher synthesized the data across the three variables: educational attainment, years of nursing experience, and size of college. For each research question and subquestion, the analysis across the groups sought to identify the trends and patterns from the faculty member's responses.

Overall, the participants openly shared their thoughts and feelings to the questions in the Interview Schedule. At times there were deep sighs and long pauses, some stated they had not thought about the questions and their feelings. Many of their thoughts and feelings mirror those of other nursing faculty in the literature. Some may represent new thoughts or ideas. The Research Questions 1-7 were summarized for each question and subquestion in this section. No further summary will be presented here. 


\title{
CHAPTER V \\ DISCUSSION AND CONCLUSIONS
}

\author{
The Study in Brief
}

Fugate and Amey (2000) found that a significant number of educators never planned to enter the ranks of higher education. Some individuals discovered the role after they prepared for another career and ended up in education as seasoned professionals possessing skills other than those that are necessary for successful faculty members. Astin (1984) found that early childhood experiences play a role in the selection of a career. Young children practice and model careers as a means of exploring the elements of the career itself. Studying about children and young adults envisioning themselves in a profession, one may hear the child say the she/he wishes to be a nurse, or maybe a teacher. However, the attraction is not there for the child or young adult to say, "I want to be a nurse teaching other nurses."

Most states require the nurse educator be a nurse and have several years of experience as a staff nurse. Since salaries in the practice arena significantly exceed those of academe, it typically poses a significant hardship on a staff nurse to leave the clinical role and move to the role of a nurse educator (AACN, 2003).

As the nation prepares for a crisis in healthcare and a shortage of its basic caregivers, the strategy of increasing the numbers of graduates from nursing programs would seem to be a given. Currently however, nursing faculty are retiring and creating vacant positions in nursing programs that are already understaffed for the current number 
of graduates even without expanding the nursing programs to accommodate the greater number of students. At issue is how to recruit or attract nurses to leave the clinical practice arena, obtain advanced education, and become a professor. Perhaps insights can be gained about the process of career choice by studying those already in the role. Both solutions to the recruitment of new prospects and retention of those currently in academe are possible outcomes of this approach. This continuing problem sets the central research question for this investigation, "What factors influenced the career choice of nurse educators currently teaching in Kentucky associate degree nursing programs?" Specifically, this study is designed to provide insights into the career choice of nursing educators in Kentucky.

The methodology for this qualitative research study was grounded in the literature on career choice, nursing faculty job satisfaction, retention and recruitment. These strands are brought together using the theoretical framework of Helen Astin's (1984) Sociopsychological Model of Career Choice and Work Behavior. The study builds on previous qualitative research on career choice and other quantitative research on job satisfaction in nurse educators. The empirical research questions were designed to investigate the overall research question identified above. Specific instruments, derived from the research questions, included a background demographic questionnaire and an interview schedule.

The target population included all associate degree nursing program faculty teaching in public 4-year and community colleges in Kentucky. This included four 4-year colleges and 16 community colleges as part of the Kentucky Community and Technical College System. Using a purposeful sampling method, one representative from each college was chosen to complete the two instruments, the Background Questionnaire for 
Nurse Educators and the Nurse Educator Interview Schedule.

A purposeful sampling matrix was developed to include all representatives of the sampling grid. All five of the educational attainment categories: (a) Less than a Master's, (b) Working on a Master's, (c) Master's, (d) Working on a Doctorate, and (e) Doctorate were included in addition to three levels of experience: (a) 0-5 years nursing education experience, (b) 6-10 years of nursing education experience, and (c) 11+ years of nursing education experience. The third sampling category was size of college based on the Carnegie Foundation classification, small or medium/large. The matrix was developed to insure the representation of all classifications of categories across the 20 programs in the state. Of note, one cell from the sampling grid was not obtained because the available faculty did not meet the educational attainment category.

The development of the research instruments was based on questions written by the researcher with assistance of the researcher's dissertation advisor and were derived from the research questions guiding the study. The instruments--the Background Questionnaire for Nurse Educators and the Nurse Educator Interview Schedule--were reviewed for clarity and content by three expert panel members. Two were from nursing education and the third was an expert in qualitative research design. After reviewing the instruments, and to provide additional feedback, evaluation forms were completed (Appendix D). The order of the questions in the Background Questionnaire for Nurse Educators was changed as a result of the feedback.

A pilot study was conducted to evaluate further the instruments and the procedures. The participants for the pilot study were nurse educators who met the criteria similar to that of the study and were willing to participate. After completion of the interview, the participants completed an evaluation form for each instrument. Feedback 
from the participants was evaluated; comments were favorable and no further changes were made to the instruments.

The instruments (Background Questionnaire for Nurse Educators and the Nurse Educator Survey) were used according to the descriptions of the methods and procedures found in Chapter III. (The instruments mapped to the research questions, the two instruments in final form, and the forms for evaluation are in Appendices A, B, C, and D, respectively.)

After approval by the Institutional Review Boards for human subjects research at both the University of Louisville and Western Kentucky University, the study began with a letter to each of the programs' nurse administrators. It detailed the study information, including the characteristics of the particular research subject as needed from that program in order to complete the purposeful sampling grid. The nurse administrator prepared a memo and contacted nursing faculty who met the specific criteria, enlisting their participation. Completed forms for participation were sent to the researcher. The researcher contacted the faculty member and arranged an interview at the convenience of the faculty member. When the researcher was notified that the available faculty did not meet the qualifications for that program's position on the grid, the researcher sent a form to the nurse administrator requesting qualifications of the faculty in relation to educational attainment and years of teaching. With this additional data in hand, the researcher was able to shuffle the programs on the grid so that 19 of the 20 spots were filled with a faculty member who met respective requirements for size of college, years of experience, and educational attainment. The final spot on the grid that could not be filled was for a subject who matched as follows: Doctorate, 6-10 years of experience, small college. 
The final sample of 19 faculty, one from each of the 19 programs, were contacted by the researcher. All of these individuals agreed to participate in the study. Each interview session lasted approximately an hour. Subjects completed the Informed Consent and the Background Questionnaire for Nurse Educators prior to the interview. Each session was audio-taped by the researcher and transcribed. Once transcribed, the interview was sent electronically to the subject to verify content. The tools used to gather the data were specifically designed to assist the researcher in analyzing the meaning of the responses, providing insights into what factors affect the career choice of associate degree nurse educators in Kentucky.

\section{Discussion}

The results of this study relate to the literature on career choice, job satisfaction, organizational climate, and expectations of new faculty. The nursing faculty members in the study were able to articulate their thoughts on the flexibility of the position and its effects on their family, their own life, and their role within the profession. The organizational climate of the college setting was more supportive and autonomous than the clinical setting. New nursing faculty were overwhelmed by the role expectations of the teaching position.

The remainder of this section is organized by the seven research questions. For each question, the findings from the Background Questionnaire for Nurse Educators or the Nurse Educator Interview Schedule are synthesized for the research question as a whole. These findings are then discussed in terms of relevant literature and replications for the field.

\section{Research Question 1}

To what extent are personal and demographic factors influential: 


\section{1.a. Age?}

From Background Questionnaire item 3, the average age of the 19 subjects was 47.3 years of age. The youngest of the group was 28 years and the oldest was 65 . Eleven of the faculty were 46 years of age and older. In the educational attainment category, the youngest group was the "Less than A Master's" and "Working on a Master's"(combined due to only one representative in the "Working on a Master's" group), with the average age 31.6. The "Master's" group had an average age of 54.7 years and was the oldest group. In the category of "Years of Nurse Educator Experience," the youngest group was the "0-5" group with an average age of 31.6. The oldest group was the " $11+$ " group with the average age of the faculty members being 54.3 . The "Small" college category has the youngest average faculty group at 47.1 years. The "Medium/Large" group had an average age of 54.3 years.

Analysis

The SREB (2000) study noted that the average age for nursing faculty was 58.5 years. The difference in ages of the sample group (47.3) and the SREB study was over 11 years. The youngest faculty member in the study was 28 years old, much younger than the average age for faculty. The JCAHO (2002) stated that 56 years of age was the average age of a doctorally prepared faculty member. In the study the average age was 49.5. The overall age of faculty in the study is younger than in the literature. This suggests additional productive years for subjects in the study sample. However, the " $11+$ " years of faculty experience category was 54.3 , reflecting the aging trend of the nursing faculty member today. This supports the literature from NLN (2006). In the present study the age range was 43 and the oldest was 65 . Although the senior members had an increased number of years of experience, they did not all have the highest educational 
attainment; four of the seven were Master's prepared. By college size, there was essentially no difference between the "Small" college group and the "Medium/Large" group. Both had an average of about 47 years. Ryan and Irvine (1994) used the term "bipolar professorate" to characterize junior and senior faculty. The generational differences in this research study support this term.

1.b. Marital status?

Of the participants in the study, 15 of the 19 faculty members were married, two were single and two had domestic partners (from the BQ4). Comparing between groups, educational attainment, years of nursing experience, and college size; the most diverse group was the "Doctorate" group for educational attainment with one married, one single, and two with domestic partners.

Analysis

The evidence on marital status for this investigation was not significantly different from other literature. Although marital status has been included as a variable in research studies, there has been little significance between being married and job satisfaction, burnout, or role transition. The research study by Winter and Munoz (2001) on the association between personal characteristics and job ratings of applicants for a faculty position at a community college determined that marital status did, however, explain the differences in the applicant's interest in the position. It could be that marital status is more important for those considering a change in employment, but once a position is secured, it makes little difference in how that job is perceived.

1.c. Current family responsibilities?

Background Question 5 raised family responsibilities. Nine of the 19 subjects had dependent children, two had adult children, and eight had no children. One faculty 
member was caring for one or more adult relatives.

Analysis

Some faculty members cited the desire to change careers from clinical positions to teaching as a means of having more time with family. However, there is no current research on this issue. Lindholm (2004) in explaining faculty career choice, identified the desire for independence as a means for choosing careers. These data on family responsibilities are suggestive, but in themselves provide no clues as to why or why not or to what extent. Astin's theory is given minimal support by the subjects' responses on the Interview Schedule for Nurse Educators. During questioning in the interview only three faculty mentioned family as one of the reasons to change careers. The one faculty member who is caring for adult relatives came to the area to care for them and as a result she entered teaching, but not exclusively because of their situation. Literature shows that the responsibility for caring for an adult parent may be a reason for leaving teaching (SREB, 2000). This clearly warrants more work.

\section{1.d. Years of nursing experience?}

Seven years was the least amount of nursing experience that any faculty member had and 40 years was the most as obtained from BQ6. Since faculty are required by Kentucky Board of Nursing to have at least two years of clinical nursing experience, this sample more than met that requirement. These nurses were experienced in a variety of clinical specialties. The average years of experience of the subjects were 23.5. As would be expected, the educational attainment groups, "Less than a Master's" and "Working on a Master's" had the least number of years nursing experience. All three faculty in these two groups had begun their nursing careers as associate degree nurses and had completed the BSN. They were still early in their professional careers. The groups with the 
educational attainment of "Master's" and "Doctorate" were close to the average number of years as a nurse: 27.6 and 27.3 , respectively. On the average, the most experienced nurses (number of years as a nurse) were in the "6-10" nursing faculty experience group. Analysis

There was a good representation of nursing experience among the faculty in this sample. With the least amount of experience being seven and the most 40 , this reflects the age range seen in most programs of nursing today (NLN, 2006). Evidence from across the 19 subjects indicates that the senior faculty member (with a great deal of nursing experience) and the young lady who may have the most up to date technical knowledge based upon her previous clinical position (but less teaching experience) may be working in the same setting. That is, these data do not support a finding of stratification but rather a mix of experience across the programs.

1.e. Years of nursing faculty experience?

Thirty-two percent of the subjects had been teaching nursing for less than five years (from BQ7). One faculty member had been teaching full time for just two months. Forty-two percent (8) of the faculty had taught from 10-20 years. The two senior faculty had taught for 28 and 32 years, respectively.

Analysis

This was a diverse group of experienced faculty members. With 10.3 the average number of years teaching, this would be characterized as a senior group. However, with $32 \%$ of the group having taught for less than five years, the range in experience from two months to 32 years provides a rich description of the generational gap in the teaching group. The literature does not address the specific years of teaching experience. However, due to the overall aging of the nursing faculty population (NLN, 2006), the 
increase in years of teaching experience would provide an expectation that this group would be more experienced in the educational process, yet may be experiencing certain barriers of their own in relation to changing technology.

\section{1.f. Mother's educational attainment?}

From Background Questionnaire 8, the "11+" group of teaching experience had the most educated mothers. One mother had less than a high school diploma, two faculty had mothers who had completed high school, one had some college, two had college degrees, and one had a professional or advanced degree. In the educational attainment grouping, the mothers of the "Master's" group had the most education.

\section{Analysis}

Astin (1984) cited the role of family expectations in the determination of career choice. If one or more parents have a college degree or may be teaching, there is a greater expectation for the nurse to choose teaching. Because the nursing profession is much more commonplace than nursing educators, there may be the expectation from the parents that daughters go into nursing, but not necessarily to teach nursing (nursing is still predominately female). In today's society, mothers have completed higher education so the value of education is increasingly taken as a given. Yet it is not clear what impact this changing demographic will have on recruitment first into nursing and then to nursing faculty. Nursing has traditionally been a profession for the upwardly mobile, with a high proportion of first generation college students. Will the supply of nurses (and then nursing educators) continue to come in a large part from first generation college graduates? Or does the demographic shift portend more nurses with college-educated parents? In Adams (1985) study the subjects' (nursing faculty) parents were generally high school graduates. This study, 25 years later, still resembles a similar educational 
level. However, more work is needed as the country's educational level continues to increase.

\section{1.f. Father's educational attainment?}

Overall, the fathers of the subjects had more education in relation to college and advanced degrees than the mothers. There were three fathers with a professional or advanced degree and one with a college degree compared to mothers (only one with a professional or advanced degree). Although the same percentage (42\%) of the fathers graduated from high school, the fathers in the "Medium/Large" faculty group had more education when compared with the "Small" college category. Analysis

Astin's (1984) work refers to the educational expectations of the parents in relation to making the choice to teach. Comparing the groups with the most teaching experience, the "11+" group had the fathers who were the most educated. Of the " $11+$ " group, four of the nurse educators had a Master's degree, one working on a Doctorate and two had doctorates. The educational pursuits of the nursing faculty may be as a result of the expectations of the fathers as well as the mothers.

Of the 19 nurse educators, only three mothers (16\%) and four fathers $(21 \%)$ had college degrees. Only one faculty member had both parents with degrees. All faculty teaching had at least Bachelor's degree; 10 of the 19 faculty were licensed as ADNs initially. Perhaps the opportunity for first generation college students to elect the associate degree in nursing may be more attainable. With the number of community college nursing programs in the state, opportunities for many students to attend close to home is a reality. In addition, community colleges are typically cheaper and admissions criteria not as demanding as that for baccalaureate institutions. McMurray and Sorrells 
(2009) noted that one of every two students in the community college setting is a first generation college student. With this in mind, community colleges recruit students to nursing programs with an understanding of the needs of first generation students.

1.g. My educational attainment?

There were seven faculty with Master's degrees, the largest number of degree type in the study as obtained from Background Questionnaire 10. Three members had BSNs and four had Doctorates. The "11+" group had the faculty members with the highest educational attainment.

Analysis

Associate degree faculty who are teaching in Kentucky have two requirements by the five year mark. The KBN requires a Master's degree within five years of the initial employment date and KCTCS requires faculty to obtain the Master's by the time they approach the promotion to associate professor. Since the BSN is the entry level for teaching with the 5-year requirement for the MSN, these members had expectations with employment to begin working on the MSN. The community colleges offer up to six credit hours free to each full time faculty member to a public university in Kentucky. Certainly this makes it easier for the faculty member to attain a higher degree. As a result, the faculty in the "6-10" group and the " $11+$ " would have completed the Master's degree. Twenty-one percent of the subjects held a Doctoral degree, which exceeds the current literature (NLN, 2006) where only 6.6 percent of the faculty held a Doctoral degree.

Overall these data reflect the fact that nursing as a profession continues to be a field that provides opportunity for social mobility with a high proportion of first generation college students.

\section{1.h. Influential person or event?}


From IS1, "Tell me when and why you decided on nursing as a profession?" The most common themes for entering the nursing profession were: (a) a relative had been a nurse or other healthcare professional (37\%), (b) an experience in which a relative had been ill or had died and who had received either positive or negative care (21\%), and (c) 16\% stated they went into nursing to care for people. Two subjects reported they were interested in other careers, but in the late 1960s and early 1970s, only certain professional careers were approved for females by the social expectations of the time. Analysis

The themes support the literature and the framework of the study. Astin (1984) proposed that expectations for career choice were shaped by "early socialization, through family, childhood play, school experiences, and early work experiences" (p. 121). In this study subjects who had relatives who were nurses or healthcare professionals were a key part of their decision to go into nursing. A subject in the study talked about playing nurse and making nursing hats when she was a little girl. Another participant reported the exposure to the healthcare field after her father owned a nursing home and her interactions with the patients were a source of enjoyment for her. Astin believed that girls, in playing with dolls and pretending to be a teacher in school, invest in genderbased expectations for caring and nurturing, not competing. As the girl begins to make decisions concerning a career, the needs for pleasure and contribution are satisfied by direct service to others. Thus nursing would be a career choice that would meet these psychological expectations.

It is important to note the two faculty members who early in life were interested in other careers. Yet 40-45 years ago only certain professional careers were accepted. All others were frowned upon. One faculty member wanted to become a lawyer, another 
wanted to be a doctor. Neither profession was highly regarded for women to seek advanced education and licensure.

IS2. What influenced your decision to become a faculty member?

The faculty reported a variety of reasons for making the change from clinical nurse to nurse educator as obtained from the interview data. The most common reason for making the change was a situation in the clinical setting based upon unpleasant occurrence/occurrences while engaged in patient care or supervisory responsibilities. Two faculty told of their family relationships where family had reinforced the decision. As educators, the parents provided both positive and negative glimpses into the life of a teacher. Two faculty had teaching roles within the clinical setting and made the transition from teaching in the hospital to that of higher education. Three faculty had relocated to the area and had been informed of job openings within the college setting. Two faculty identified a need to continue their love for teaching and learning. Analysis

The faculty responses to the career change to nurse educator reflect those ideas and concepts of Astin (1984) and Lindholm (2004). Astin related that a person's work expectation would not necessarily remain stable throughout their life time. She recognized that even drastic changes could occur in the person's lifetime. Lindholm (2004) saw career choice changes as "changes or modifications in the structures of opportunity" (p. 608) and the pursuit of an occupation satisfies the basic needs of the individual. The satisfaction of the basic needs could explain the decision for the three faculty to change careers based upon situations in the clinical setting (physical fatigue or inability to handle the stressful situations). Likewise, the realization of family social expectations in the perceived work of the parents could explain the nurse's decision to 
teach based upon exposure to the occupations. It is interesting that one faculty member negatively perceived her relatives' roles as teachers and therefore was not interested in teaching. She states, "I think I came full circle."

Faculty who identified a love for teaching and learning are satisfying one or all three of the basic needs: survival, pleasure, and contribution as identified by Astin (1984). Faculty who relocate to the college geographic area and make the decision to teach are making choices based upon expectations of the accessibility of alternate forms of work and the capacity of that alternative form of work to satisfy the three basic needs (Lindholm, 2004).

IS3. Was there a key person/situation that supported your decision to teach? In what way?

Across the groups, the faculty related that faculty currently teaching in the program, as well as the nursing program coordinator, were supportive of their decision to teach, as obtained from the interview schedule. Several cited previous faculty who taught them as being instrumental in supporting their decision to teach. To them, teaching alongside their former professors was a great opportunity. Another faculty identified a relative who was instrumental in supporting her. Analysis

The support from current or previous faculty teaching in the program mirrors the work of Lindholm (2004) who identified the role of past faculty or past college experiences in the decision to teach. Advice and support for the individual was seen as increasing the confidence of the prospective faculty member to take the position. Lindholm identified a small number of people who came into teaching in what she described as "accidental academics" (p. 618). The opportunity to teach and the 
subsequent employment lacked any specific reason for the decision. The random decisions were very small in number. Perhaps the best illustration of this is one faculty member who had three careers outlined in her life; teaching is her second career and is a job from which she derives pleasure.

The results also reflect the work of Grandjean et al. (1976). Faculty rated the opportunity to work with supportive colleagues as a source of job satisfaction in relation to professional autonomy in nurse educators. This characteristic was ranked second among all characteristics with the "opportunity to become a good teacher" as the most important to the subjects in the study.

\section{1.i. College Classification--Small or Medium/Large?}

The classification of the 19 respondents was done by the researcher. From institutional criteria using the Carnegie Foundation Classifications of Institutions of Higher Education (n.d.), the 20 public colleges with associate degree nursing programs were surveyed. One college was not included in the study because the faculty currently teaching in the program did not meet the descriptor designated for the study. This resulted in eight colleges in the Small category and eleven in the Medium/Large classifications.

\section{1.j. Other?}

IS4. Anything else you would like to add about the personal or background factors that influenced your decision to teach?

Respondents interpreted this query as a continuation of IS3, above. One faculty member captured the passion observed by the researcher when asked the question. "I love teaching, I love teaching.... I haven't regretted one moment even though it seems very time consuming." Another's response is similar in that she loves teaching and thought she 
should have always been a teacher. Still others responded that they loved the student interaction, loved putting together a lecture, and enjoyed being with students.

Analysis

Lindholm (2004) describes the passion of the subjects as she interviewed them for her research. The overall "fit" of the faculty member to the role was verbalized. The same passion and feelings of "fit" were evident in the statements of the faculty as they discussed their thoughts about whom or what influenced their career choice. Despite the limited work in the nursing/nursing faculty literature on love of teaching, love of students, and rewards of working with their nursing students, the findings in this study do replicate, with uncanny verisimilitude, the classic work by Lortie (1975) on the overwhelming importance of psychic rewards in the career satisfaction of public school teachers.

\section{BQ1. Gender?}

Of the 19 subjects, there were 18 females and 1 male. The male was in the categories of Doctorate, 6-10 years of teaching experience, and a Medium/Large college. Analysis

With just five percent male subjects in the study group, this reflects the current trend of males in the nursing profession. NLN (2006) reported that in the southern region the male nurse faculty population teaching in associate degrees was seven percent. This was the most of any of the regions.

\section{Research Question 2}

In what ways do educational degree requirements for faculty positions:

2.a. Influence decisions to become a faculty member?

IS5. What was your original educational level for licensure? Did this influence 
in any way your eventual decision to teach? How and why?

From the interview schedule, fifty-three percent of the subjects completed an associate degree for their initial licensure. Many cited the close proximity of the college to their home, or one of their relatives had graduated from the program. Fourteen of the nineteen subjects were the first generation college students in their families. Of note, there were three faculty to complete a Diploma in Nursing. These were hospital sponsored programs with an emphasis on clinical skills. There are currently none in operation in Kentucky. One graduate from the program cited the reputation for the excellence in nursing skills as a reason for her enrollment. The other two graduates were 53 and 58 years of age with the third much younger at 30 . There were six subjects who completed the BSN for initial licensure. One faculty person identified the need to return to school to get an advanced degree as an influence for making the decision to teach. Analysis

Six of the eight subjects who obtained the associate degree first stated that they realized the ADN was not enough education and to increase the opportunities for additional responsibilities in the clinical setting, the need for additional education was a necessity. This did not influence their eventual decision to teach. This may be a result of the KBN requirement for two years of clinical experience before teaching. Also, none of the faculty made the decision prior to, or during their initial nursing education to teach. The requirement by KBN for faculty positions is a BSN with a Master's within five years (Kentucky Board of Nursing, n.d.). The 14 of 19 who were first generation college graduates is consistent with nursing as a gatekeeper profession with respect to social mobility.

IS6. What was your original educational level when you started teaching? Did 
this enter into your decision to teach? How and why?

Eleven faculty had BSN degrees when they began teaching and eight had Master's degrees. Only one faculty member returned to school to complete additional coursework because of a desire to teach. One faculty member completed the Master's and then made the decision to seek a teaching position because "I've done nothing with my education."

The group "Working on a Doctorate" had the faculty with the most Master's degrees (5) upon initial employment in teaching. The "Medium/Large" college category had the most new faculty with Master's (6) in comparison to the "Small" college category. Analysis

The minimum credential for employment in associate degree nursing programs is a BSN. Those faculty with a BSN must complete the MSN within five years from initial employment. Faculty with an MSN at initial employment have met the criteria for teaching in an associate degree program. Nurses with a BSN who elect to return to complete an MSN may experience barriers to successful completion. Likewise opportunities for the "Medium/Large" colleges to hire Master's nurses initially may be greater with a larger pool of employed nurses in the more metropolitan areas that they serve. With the increase in Master's and doctoral programs in Kentucky, the barriers to completing additional education degrees are currently being reduced.

2.b. Influence career trajectory (tenure and promotion) and retention?

IS7. What are your college's requirements for terminal degrees for promotion and/or tenure? To what extent did this influence your decision to teach?

Requirements for a terminal degree vary according to the college where the 
program is located. In the 4-year colleges, the doctorate is the terminal degree. In the community colleges, 12 faculty reported that the Master's was the terminal degree. Two faculty from the community college system reported that the Bachelor's was the terminal degree.

Two faculty members reported that tenure was no longer an option at their college. One faculty member in the 6-10 year experience group reported that her hiring cohort were the last to get a tenure-track position in the college. No one indicated that a terminal degree or tenure track position influenced their decision to teach. Analysis

The findings support the work of Winter and Munoz (2001) who studied the recruitment simulation for job applicants using job tenure as a variable for characteristics leading to the attractiveness of a community college position. Job tenure was not significantly correlated with ratings of attractiveness of the faculty position. Kennerly (1989) studied nursing faculty job satisfaction and found that for the population he sampled, there was no significance between job tenure and job satisfaction.

Thus in general, the results of this study mirror the wider literature: requirements for a terminal degree are taken as given, required after becoming a faculty member but do not have much effect (if any) on decisions to become nursing faculty. But this is a restricted population. By definition, everyone who is in a faculty position accepted these employment standards. Unknown is whether some clinical nurses may have been interested in the career switch but decided against it because of the necessity of higher degrees. Answering that would require a separate study focused on clinical nurses and their potential interest in becoming nurse faculty.

Research Question 3 
To what extent is the work environment in academia (compared to clinical nursing) influential:

\section{3.a. Flexibility?}

IS8. Can you compare the flexibility of your teaching position within the college setting and your previous clinical position? Do you consider this a benefit or burden of teaching?

Across the groups the faculty overwhelmingly felt that the flexibility of the teaching position was much greater than in the clinical position. The unpredictability of the clinical environment was a common concern. Coverage for all shifts, weekends, and holidays in the clinical setting made it hard on family life. However, one of the newer faculty members shared a concern that many hours of upfront preparation have been overwhelming. Another faculty member felt the pressure of being on call for 24 hours a day. More common was the greater sense of freedom (lots of work but more flexibility) as reflected in one respondent with " $11+$ " years of experience, for whom it meant the opportunity to develop a social network and to find a spouse.

\section{Analysis}

There is an overall consensus that the flexibility of teaching is greater than in the clinical setting. For each subject it meant something different. This supports the work of Astin (1984) and Lindholm (2004). Lindholm identified the "personal needs" for autonomy, independence and individual expression and the general lure of the university environment. Linked closely to a strong need for autonomy was an expressed passion for the 'spirit of individuality' and 'sense of personal independence' that faculty work affords" (p. 611). Lindholm continues, "Having a flexible schedule was viewed as highly appealing characteristics of professorial work" (p. 612). The findings in this study clearly support that theoretical stance. 
Garbee and Killacky (2008) studied nursing faculty and satisfaction in their role with intent to stay in the position. Flexibility in the nursing position was ranked second to being part of student success when subjects were asked to identify three to five factors contributing to the faculty member's satisfaction from work. Thus, from the overwhelming response of the 19 subjects in this study, this was an important factor in role satisfaction.

IS9. What is your sense of this flexibility now (as an experienced faculty member) compared to when you started? What was your perception of this at the time when you first started teaching? Was it influential in your decision to change your career? Why?

The expectations of faculty members during the initial period of employment were expressed by many during the interviews. The additional work necessary in preparation for the classroom and clinical was expected but other responsibilities demanded additional time. One faculty member spoke of "rose-colored glasses." She had been told there was flexibility but in her first year the preparation, grading, advising, and new faculty orientation activities still have been overwhelming. For another faculty the time for test preparation and grading came after class. Analysis

This shared experience of overwhelming expectations supports the works of LaRocco and Bruns (2006) that identified dimensions of academia and work life. The subjects in the LaRocco and Burns research had difficulty in finding a balance in their work life and home life. It was reported that only a few of the subjects were able to feel confident about their ability to teach and meet all of the responsibilities of the role.

In the Garbee and Killacky (2008) study, faculty were to identify three to five factors that contributed most to dissatisfaction with the teaching role. The main theme 
identified regarding dissatisfaction was time demands. The time demands of the new faculty seemed to have been not what was expected.

3.b. Collegiality?

IS10. Can you compare the collegiality of your teaching position within the college setting and your previous clinical position? Do you consider this a benefit or a burden of teaching? Why?

Overall, the faculty responses demonstrate that positive collegiality was also present in the clinical setting. Several faculty spoke of a difference in clinical versus academe, but $53 \%$ responded that collegiality was present in both environments. One member stated that the positive relationships were valued at the college; the nurse educator had experienced some very rewarding relationships in the clinical setting as a staff nurse and was pleased with the atmosphere of the college. One faculty member spoke of the change in the collegiality as the college changed governance from one college system to another. It had become a more business-like atmosphere. One faculty member spoke of a "sisterhood" in the faculty relationships within her program. The term "family" was used in reference to the relationship of the faculty outside the college. Analysis

In the study by Garbee and Killacky (2008) the faculty identified three to five factors that contributed to job satisfaction. The collegial environment was ranked fourth in importance. "Faculty colleagues" was ranked third with $15.5 \%$ of the respondents rating this as important. Thus, the somewhat neutral response from the nursing faculty in the current study reflects the same results.

IS11. What is your sense of this collegiality now (as an experienced faculty member) compared to when you started? Was it influential in your decision to change your career? Why?

There was a period of adjustment for the nurse educators as they settled into their 
new role in academe. The overall adjustment for four of the faculty was affected by the overall climate of the faculty within the nursing program. Problems occurring within the program with students, student performance, and educational responsibilities provided concerns for the novices. One faculty member from the " $11+$ " years of nursing faculty experience voiced concern about the change in leadership at the division chair level that had a negative impact on the morale of the nursing faculty. One who had extensive years of teaching experience came to the new setting and conveyed that she had to gain the trust of the other faculty. Another experienced faculty member related that a lack of collegiality was a reason for leaving the past teaching position. Five faculty stated that it was influential in their decision to change their career from clinical practice to higher education.

Analysis

LaRocco and Bruns (2006) studied new nursing faculty. They identified three dimensions as part of a new faculty member: entry into academia and work life, participants' knowledge of the college, and the perceptions of their relationships with colleagues and supervisors. The support most valuable to the new faculty was from colleagues. In this investigation, the responses from the nurse educator group support the findings of that study.

3.c. Organizational climate?

IS12. Compare the organizational climate of your teaching position within the college setting and your previous clinical position. Do you consider this a benefit or a burden of teaching?

Sixty-three percent of the nursing faculty members responded that organizational climate was a benefit of teaching. A sense of autonomy was conveyed within the college setting as opposed to the management driven or union controlled standard procedure in 
the clinical settings. One faculty compared the "hands-on" nature of the program coordinator with the managers at the hospital. In the hospital setting there was a lack of communication and a spatial distance between individuals.

Analysis

The findings from the current study support the work of Johnson (2001) who found that organizational culture of ADN faculty was a statistically significant contributor to faculty empowerment as well as job satisfaction. The autonomy of the teaching role is also identified in the works of Lindholm (2004) as a source of faculty satisfaction.

IS13. What is your sense of this organizational climate now (as an experienced faculty member) compared to when you started? What was your perception of this at the time when you first started teaching? Was it influential in your decision to change your career? Why?

Four faculty agreed that the organizational climate of the college was influential in their decision to teach. Experience with a negative climate by two faculty created a source of concern. Poor leadership within the nursing program can lead to discord and unrest. From the qualitative nature of the responses, these 19 nurse educators provided a depth of understanding about how and why autonomy is important to them, a sense missing from the predominately quantitative studies in the broader literature. Poor dynamics within the college can create an air of uncertainty for new faculty who may reconsider that the college role is not for them. In general, however, the faculty in this study had positive perceptions about the organizational climate of their college. Analysis

Although several of the faculty met with discord within the climate of the college, the support they received from experienced faculty seemed to sustain them. Despite the 
doubts in the decision to change careers, the overall support from within the nursing program seemed to have provided the positive interaction for them to stay. This supports the work of Siler and Kleiner (2001) who studied the experiences of new faculty. The study centered around the transition into the teaching role. One of few qualitative studies in the broader literature besides the current work, the areas that emerged included assistance from colleagues as a source of expectation in the new role.

\section{3.d. Prestige/status?}

IS14. Can you compare the prestige/status of your teaching position within the college setting and your previous clinical position? Do you consider this a benefit or a burden of teaching? Was it influential in your decision to change your career? Why?

Prestige/Status of the teaching position brought both positive and negative responses. The group that regarded the position with the most prestige/status were those with " $0-5$ " years of teaching experience. Four out of seven regarded the position as having prestige/status. Two faculty who were in the "Working on a Doctorate" group stated that the prestige/status of the position was influential in their decision to teach. Two other faculty responded with negative experiences when taking coursework alongside faculty from the higher level programs. One faculty member considered being a nurse more prestigious than a faculty member. Analysis

The faculty with the least experience in teaching and the closest to the clinical position generally characterized the teaching position as having the most prestige and status. Although research does not identify prestige/status as a means of recruiting faculty or job satisfaction as identified by Grandjean et al. (1976), the faculty did regard it as important with five faculty agreeing that it was somewhat influential in the decision to 
teach. However, this sentiment seemed to wane over time. As faculty gained greater experience in higher education, they were more likely to become aware of the generally lower prestige/status of associate degree colleges compared to the spectrum of higher education that includes not only 4-year institutions but also graduate programs ranging up to research universities with doctoral programs.

IS15. What is the sense of this prestige/status now (as an experienced faculty member) compared to when you started? What was your perception of this at the time when you first started teaching? Was it influential in your decision to change your career? Why?

Four of the subjects in the " $0-5$ " year group answered that prestige/status was an important factor in their decision to change careers. The members of this group identified a new appreciation for the role of the faculty member. They were unaware of all of the responsibilities related to the role. As the years of teaching experience increased, faculty were less concerned with prestige/status of the teaching position. One faculty member related the loss of prestige/status of a nursing program as it is merged from a community college department into the school of nursing. The concern was the loss of the history and meaning of its identity (formerly) as an ADN program. Analysis

Prestige/status seemed to be important for those faculty members who had just left the role of clinical nurse. Perhaps as the faculty transitioned to the teaching role the prestige/status of the role diminishes. The responses for IS15 mirror those from IS14. Relevant are the place of associate degree institutions in the hierarchy of higher education as well as the prestige of nursing compared to other professions. The literature on prestige levels in higher education and for comparative professional prestige are both relevant here. 
3.e. Other?

IS16. Can you think of any other factors related to work environment that influenced your decision to change from clinical nursing to faculty status? How and why?

Three themes were identified from the responses in relation to work environment. The younger faculty regarded flexibility as the factor influencing the change from clinical environment to the college setting. For the faculty who were older, the physical stress of the clinical environment was a factor. For all groups the desire to be with students and to be a lifelong learner was extremely important. The latter especially described the passion of the nurse educators during the interview portion of the study.

Analysis

The physical challenges related to the clinical setting and the overall stress that several of the faculty endured prior to career change could both be considered as survival needs, according to Astin's (1984) framework. The role of nursing faculty seemed to meet these needs better than clinical nursing, especially as individuals got older. Once physical exhaustion became a concern, the pleasure and contribution needs were also not being met. Pleasure of the work setting comes from the engagement of the activity and the satisfaction on completion of the work. The pleasure was no longer a part of the work environment. The findings on this issue for this study also reflect new information regarding the work life of nursing faculty.

\section{Research Question 4}

To what extent are pedagogical considerations influential?

4.a. Love of teaching?

IS17. Think about your reasons for changing from the clinical environment to teaching. How do you feel when you teach? 
Across the groups the faculty shared their feelings about teaching. From the faculty member having taught for two months to the senior member who had forty years of teaching, the passion and the joy, was very evident. From "empowered" to "stimulated" to "I am charged up maybe a little too much," these words came from the subjects as they thought for a moment upon hearing the question and then began to explain their feelings. The message came from across the sample, regardless of subcategories they filled.

Analysis

The passion of the subjects as they explain their love of teaching and nursing is supported by the works of Lindholm (2004). Lindholm wrote:

In listening to the participants' reason for pursuing faculty careers, the categories of career choice motivation that Astin (1984) delineates--survival, pleasure, and contribution--clearly was evident. This was particularly true in terms of participants' need for pleasure as a driving force for pursuing faculty work. Nearly without exception, those interviewed spoke passionately about their jobs. For many, teaching is also a highly rewarding component of faculty work. The degree of pleasure that study participants derive from their work-related pursuits is substantial enough to lead some to conceptualize their professional activities as more analogous to play than work. (p. 625)

Further, these overwhelmingly positive and consistent sentiments about love of teaching and its immediacy as a form of psychic rewards mirror precisely the classic work of Lortie (1975) on what drives public school teachers, i.e., the rush, the sense of gratification, the satisfaction that comes from interacting with their students.

IS18. When you started your career as a nursing faculty member, did you see teaching as a plus or a minus? Why?

Seventy-nine percent of the faculty members responded that teaching was a plus. Three responses centered on the opportunity for advancement of the individual faculty member within the profession. Several included the opportunity to teach and advance the 
knowledge of others. One faculty member saw teaching as a positive when they saw former graduates caring for patients in the clinical setting. Two faculty members in the "Doctorate" group reported that they had no expectations for the job and were neutral as to an opinion if teaching was a plus. One faculty member mentioned that she only appreciated the time off during the summer.

Analysis

The positive association with advancing the knowledge of others is reflected in the study by Garbee and Killacky (2008). "Being Part of Student Success" was ranked by 316 participants as the most qualitative factor contributing to job satisfaction. Faculty satisfaction is reinforced when seeing graduates care for patients. The Garbee and Killacky study is one of the very few qualitative studies examining job satisfaction. It is important to note that qualitative studies found this factor by picking up the more subtle cues that quantitative approaches cannot.

4.b. Lifelong learning?

IS19. Talk about the extent that you have always loved or disliked school. Did this have any influence on your decision to enter teaching? In what way?

Eighty-nine percent of the faculty reported they liked school or liked reading. One faculty member related that she would never be too old for classes. Faculty who were in the "Working on a Doctorate" group related that they had always enjoyed school and continued to return to school for advanced education. Although faculty enjoyed school, many did not believe that it influenced their decision to enter teaching. One faculty member related her decision to teach with a love for school. Analysis

The question of school and its influence on the decision to teach has not been 
addressed in any of the studies included in the review. Although there have been qualitative studies examining the characteristics of job satisfaction among nursing faculty, there is little data to support the faculty perception of school and its influence on the decision to teach. This finding represents not only new information for the field but also a potential strategy in the recruitment of nursing faculty from the ranks of clinical nurses. Although this particular aspect of higher education may not appeal to all clinical nurses, it may spark interest in some who are missing that aspect of their personal identity in their professional career.

4.c. Possibility of influencing the next generation of nurses?

IS20. How would you describe the rewards of teaching? Has your sense of this changed since your started this career? In what ways?

Faculty across all groups consistently responded they were more satisfied in the classroom with the students. It was rewarding when the student demonstrated growth in the knowledge and skills. The faculty members reported they were rewarded when the student was successful on the licensing exam and was observed giving care to patients. Commonplace were faculty members who used the words, "they like to see the light bulb go on."

Analysis

Garbee and Killacky (2008) studied faculty intent to stay in the teaching position. A portion of the study was quantitative in design. At the end of the last questionnaire faculty were asked to identify three to five factors that contributed most to the satisfaction of their job and the same number that led to dissatisfaction of their work. The factor rated the highest was "Being Part of Student Success." This would best characterize the faculty responses in the current study, i.e., the greatest reward came from student success. 
Faculty verbalized a sense of reward when the student continued to advance their education. For one faculty member, it was seeing the former student five years later still in nursing and "loving it."

IS21. How do you feel about your relationship with your nursing students?

Nursing faculty members were concerned about maintaining a professional relationship yet being approachable in order to meet the students' needs. Being a role model for the students was a common statement. Most faculty stated they had developed a positive relationship with their students. Faculty verbalized the difficulty in maintaining a professional relationship and being in tune with the social networking sites. An open door policy was a method of being approachable for many. In general, however, despite their awareness of the need to balance how close to get to their students, most felt they resolved this issue professionally.

Analysis

Milosheff (1990) studied community college faculty and factors that contribute to job satisfaction. One important factor of that study was the satisfaction with teaching students who were well prepared. However, social interaction with students was not statistically significant. Working with students who were not prepared and lacked good study skills was seen negatively by the faculty member. Likewise in the Garbee and Killacky (2008) study, faculty rated student attitudes as a source of dissatisfaction. The lack of preparedness was not included in the discussion of students in the current study.

IS22. To what extent are these feelings connected to the immediacy of the classroom? To long term issues like the nursing shortage? Other?

The subjects in the study were focused on the immediate concerns of the student. Many of them noted that the nursing shortage was important, but more important was the 
development of a caring, efficient, and highly skilled nurse. Helping the student attain their goal of becoming a professional nurse was important for the faculty. One faculty member cited replacement of a new nurse for one retiring, "one replacing another on the frontline of healthcare." Another concern was the expectation of the healthcare providers and the capabilities of the new graduates. "We produce novices, they want experts." Taken across the set of responses to this question, the 19 respondents in this study overwhelmingly focused on their immediate connections to their students, their love of teaching, and the extent that this is what kept them going more than anything else. Analysis

Garbee and Killacky (2008) found that the faculty were most satisfied with having a part in the success of the student. The opportunity to participate and "to give back to nursing" was important to the faculty in their study and very much so in the current study. More generally, the study of public school teachers by Lortie (1975) establishes the relationship between the teacher and the student and the satisfaction of having an impact in the success of the student. Lortie's study focused on the teacher perceptions of the profession and the immediacy of the short term relationship with students in the classroom. The relationship with the students was tantamount to the psychic rewards of the teachers. The current study captures that same dynamic for these educators. The interview subjects had an ongoing relationship with the students and the more abstract resolution of the nursing shortage and the impact of their role in resolving the issue was not central to their focus of the job. To the researcher's knowledge there have been no parallel studies in nursing based upon Lortie's work.

4.d. Content knowledge and skills?

IS23. How do you view the intellectual challenges of staying current with the 
knowledge about your field? Did this have any influence on your decision to enter teaching? In what way?

The nurse educators were very cognizant of the need to stay current with the knowledge in the field of nursing as well as that of education in general. One faculty member acknowledged that the need to stay current was a factor in the decision to teach. There was no difference among the subgroups related to the necessity to maintain the latest knowledge in the field. One faculty member mentioned that the knowledge base is changing so rapidly and using additional resources for teaching are necessary, since the textbooks may not contain the most current information.

Analysis

Nurses are required to complete professional development hours for licensure renewal in Kentucky and staying current may have been recognized as a necessity for the faculty. Nursing faculty in other states may not have the same re-licensing requirements. However, maintaining knowledge of the ever-expanding field of nursing and medicine is a standard necessary for nurses and other professionals to maintain proficiency in their profession. Being able to provide the most up-to-date information to the nursing students is a responsibility of the faculty role.

4.e. Other?

IS24. Can you think of any other factors related to pedagogical considerations that influenced your decision to change from a clinical nurse to a faculty member?

Several faculty members stated they did not have a response for this question. Those that did provided insight into their enjoyment in the use of active teaching strategies in the classroom and clinical setting. One faculty member responded that it was a continual challenge to provide students with the latest methods for delivery of course 
work. Another faculty member reinforced the application of knowledge, "I am going to teach you about it here, but I want to see you put it into practice."

Analysis

Nursing faculty strive to provide interactive learning strategies for students with the constant reinforcement of the application of knowledge. Feeg (2004) noted that teaching nursing was demanding work. The nurse educator should have knowledge and ability to prepare the student beyond just the mastery of clinical nursing skills. AACN (2003) conducted a study to examine faculty members and retirement within community colleges. In addition to information on retirement planning, the faculty rated 21 characteristics and skills faculty should possess. Important skills included preparing material, presenting it effectively, and being able to capture the student's attention and provide for interaction. These findings from the field are reinforced by the current investigation.

IS25. Which of these would be the most important factor in your decision to make the change? Why?

Faculty members across the groups agreed that flexibility was the most important factor in their career choice. Four other themes were important to the faculty. Interaction with students provided an enhanced sense of satisfaction and emotion. Collegiality, unlike that in the clinical setting, seemed stronger among these nurse educators where support from experienced faculty was extremely important for faculty new to teaching. The climate of learning was important to the experience of the student and to the psychic well-being of these faculty as they watched the "light bulb" reaction and other "aha" moments. For some, the benefits of teaching, specifically the retirement opportunities, were very important in their decision to change their career to teaching. 
Analysis

This climate of learning is supported by Lindholm (2004) in her study on career choice. Lindholm used the same actual term, "seeing the lights go on in people's heads" (p. 617), which was reflected repeatedly in these 19 interviews. Lindholm continued, "Others underscored the personal satisfaction they first felt, and still experience, in knowing that they have touched students' lives through their teaching, both by challenging them intellectually and by helping to broaden their knowledge and understanding" (p. 617). Certainly, the views of teaching and actions expressed in this qualitative study can be characterized as an altruistic act on the part of these nursing faculty.

\section{Research Question 5}

What are the expectations for "being nursing faculty" when first hired regarding:

5.a. Teaching load?

IS26. Think back to the first days of your teaching job. What were the expectations with respect to teaching load? Did this influence your decision to enter higher education? In what ways?

Across all groups the faculty repeated they were overwhelmed by the entire process. Some faculty members received a reduced load; others were assigned to teach with an experienced faculty member. With a reduced load and additional support, the faculty members related that this was extremely valuable in their adjustment to the teaching role. A faculty member who experienced a heavy teaching load with little assistance wondered if the right decision had been made to change careers. From the group responses, $47 \%$ of the faculty perceived their first teaching load as being heavy with little to no support.

Analysis 
Nugent et al. (1999) studied how confident new faculty were in the teaching skill areas in relation to educational experience and years of teaching experience. The greatest amount of confidence was in the clinical skill domain. The least confidence was in the domains of course preparation, preparation of exams, and methods of instruction. The researchers suggested that experienced faculty could provide much needed assistance to guide new faculty and to mentor them. Orientation for new faculty should consist of socialization of the faculty to the college setting as well as the classroom and teaching responsibilities of course preparation, examinations, clinical evaluation, and facultystudent relationships (Nugent, 1999). This preparation for teaching serves to increase self-efficacy in the successful transition to the nurse educator role.

These results are similar to Siler and Kleiner (2001) in their study to understand what new faculty experience early in their career. Seiler and Kleiner found that faculty as members increased the level of preparation to meet the responsibilities of the position, the more confident they became. Comments from faculty in the current study support the previous work. Faculty noted that after the first semester and with lecture content developed, the demand on their time declined. As the faculty continued to the next semester the opportunity to repeat their prepared lectures and work with the students was a confidence booster. For some faculty, courses are repeated every semester and the opportunity to present the same material the second time makes the adjustment easier.

\section{5.b. Service?}

IS27. In relation to internal and external service to the college, how did your expectations and those of your supervisor differ? How were they similar?

Internal and external service is a part of the responsibility of a community college faculty member and for some faculty in 4-year colleges. Internal service was assigned to 
them. Faculty were given more latitude to choose appropriate activities. But, as faculty stated, the choice of activities varies by college. Three faculty who had moved from one college to another found there were differences in what was acceptable. Newer faculty in the research study typically had not been aware of the additional responsibilities. This had also been true of more experienced faculty during their first year. Those that came from a clinical position reported they had no expectations for internal or external service. All faculty received information about additional responsibilities during an orientation process with the program coordinator or supportive faculty. At least one faculty member stated that the requirement for external service was a burden on teaching. Analysis

Fugate and Amey (2000) examined community college faculty early in their careers. The first years of their career, according to the study, the faculty member was assigned to a mentor or advocate. Expectations for community service came during the second year of the appointment. However, faculty in this research study, according to the Nurse Educator Interview Schedule, were required to assign themselves some form of external service within the early months of the first year. According to Fugate and Amey, the greatest transition to the faculty role came between the first and second year. This was contradictory from reports of the nurse educators in this study as the transition was most difficult in the first year.

Choudry (1992) investigated the expectations of new faculty and their supervisors in relation to roles of new faculty within the college setting. Of 96 competencies desirable for the beginning faculty member, the sub-roles of teaching, practice, research, and service showed agreement between the two sets of faculty. The faculty members in this study had the same expectations that service was a responsibility of the faculty. This 
may be the case as faculty, after the period of orientation, accepted their role to perform community service as part of the faculty agreement for evaluation.

5.c. Scholarly writing and research?

IS28. Early in your career, what were the expectations of scholarly writing and research? How did that differ from those of your supervisor?

Faculty from the community college system reported that they were not expected to engage in scholarly writing or research. Faculty from the 4-year colleges with associate degree programs were strongly encouraged to participate. Some community college faculty stated that they were encouraged to present at national or state conferences and they correlated that with scholarly writing.

Analysis

In the Lindholm (2004) study, during interviews of full-time faculty in a public research university, it was noted that they sought pleasure by engaging in tasks associated with "professorial" work. To them it was teaching and research. No participants in that study mentioned service; according to Lindholm it was the third major component of traditional faculty work. Lindholm proposed that the faculty in the study excelled at both teaching and research and it was not a burden to them in their role. Since the educators were from a 4-year college, the teaching and research would be the major responsibilities.

NLN (2003) addressed scholarship for nursing faculty. From a national survey, it was identified that there is a considerable amount of role strain in relation to the ideals of faculty responsibilities. In the NLN study, nurse faculty identified the complex role of a nurse educator and the lack of available time to engage in research, scholarly activities, service, or clinical practice. Surveyed nursing faculty sought assistance from the NLN to communicate these difficulties to institutions in hopes of altering the guidelines of 
service, scholarly activities and research. In the current study, concerns of these 19 nurse educators focused on service responsibilities but not scholarly writing. Apparently, the distinction is the type of institution which the faculty are employed. For the most part, 2year institutions focus on teaching (first), then service, with little emphasis on scholarly responsibility. For this study 15 of 19 were employed in community colleges. It is likely that a parallel investigation to this study with BSN nursing faculty would produce results similar to the NLN (2003) work, because of greater emphasis on scholarly work in the 4year institutions.

5.d. Earning tenure and promotion?

IS29. How much of a concern were the requirements for earning tenure and promotion when you decided to become a faculty member? In what ways?

The newest faculty members to the programs were not assigned tenure track positions but had been informed of the promotion process. Significant to the process, one faculty member was informed during her pre-employment interview that the position came with tenure. After being hired she was informed that the position she had assumed was not a tenured position. There was some concern with the tenure process, as being denied tenure would cost the faculty member their job. A faculty member in the " $6-10$ " group who recently was awarded tenure saw it as a positive to be able to advocate for students and peers. For those who had experienced the process of promotion, the actual verification of activities seemed to be a source of anxiety. Faculty related that there was little knowledge about the tenure and promotion process prior to accepting a position. Most had received information about the process from a mentor, the program coordinator, or at an orientation session with other new faculty. Analysis 
As community colleges struggle with retaining tenure, faculty in this study seem to have little emotional investment in the process. Some saw it as positive, others as negative. Even for promotion, the faculty members viewed it more from keeping the documents rather than to verify work done. In the faculty satisfaction survey by NLN (2005), over $86 \%$ of the faculty reported that the requirements for promotion and tenure were clear, but the process was less clear. As in the current study, some faculty supported the tenure process, others did not. The NLN study called for opportunities for recognition and promotion providing financial incentives to the faculty. Fifty-four percent of the nursing faculty surveyed viewed the promotion and tenure in their college as fair. In the current study this was not a concern.

Faculty in the study seemed to be confused about the promotion and tenure process. Several community colleges have reviewed the tenure process and changes have been made to the tenure track positions of newly hired faculty. Some technical program faculty are being hired to positions in which tenure is not an option. As some faculty mentioned during the interview, there may be an inequality within the faculty of a nursing program.

Within community colleges, faculty are hired with Master's degrees and may never attain the doctorate. With the purpose of community colleges primarily focused on teaching and service with the little attention to scholarly productivity, the $\mathrm{PhD}$ may be less important as a terminal degree. However, wider trends in nursing generally reveal an increasingly greater emphasis on the doctorate for nursing faculty (Penn et al., 2008) so that even in community colleges, teaching nurses may be expected to complete the terminal degree without the expectation of publication.

\section{Research Question 6}


What are the primary barriers that you experienced in becoming a nurse educator?

6.a. Student evaluations?

IS30. Thinking back to your early days as a nurse educator, relate your knowledge of student evaluation. How confident were you? Did this enter into your decision to enter teaching?

When asked this question, faculty responded after periods of thought and perhaps with a painful memory of the process. The question was asked to attempt to understand the confidence level of faculty in evaluating students; however, the respondents shifted to students evaluating faculty and the effects of a bad evaluation. Even new faculty spoke of their concerns. Perhaps the experiences of senior faculty had been related to them and there were some pre-conceived ideas. The discussion of students evaluating faculty reverberated across the groups, despite differences in years of experience, illustrating the importance of the topic to them.

Faculty across all subcategories were not confident about student evaluations. Even senior faculty were concerned about the process of student evaluations and how devastating they could be if the group evaluating the educator is very small. One faculty member was concerned about how two people can see the instructor so differently. In the Nurse Educator Interview Schedule, a younger faculty member stated that evaluation in clinical is based upon job performance, not patient satisfaction. Analysis

Nugent et al. (1999) studied teacher self-efficacy across four domains: course preparation, instructor behavior, evaluation and examination, and clinical skills. According to that study, faculty members were most comfortable in evaluating clinical skills. Formal education courses did not increase the comfort level of teachers in the three 
domains of course preparation, instructor behavior, and evaluation and examination.

Faculty who participated in the Nurse Educator Interview Schedule saw student evaluation differently than the activity of evaluating students. The faculty saw it as the student evaluating them. There was a negative response when discussing the role that evaluations have in the performance evaluation process. The literature seems to be lacking in this area of orientation for new faculty. Handling of students seemed to be a concern for the group of faculty members in this study. Several faculty saw a negative evaluation as a means for improvement and the faculty moved on from that point. Others saw it as a no-win situation.

\section{6.b. Clinical evaluation of students?}

IS31. In the early days as a nurse educator, relate your knowledge of clinical evaluation of students. How confident were you? Did this enter into your decision to enter teaching?

The newest faculty members related during the interview that they felt very comfortable in the clinical setting with the students. Several had been preceptors in the clinical area and were comfortable in evaluating them there. Typical was one faculty person who commented that she was more comfortable in the clinical area than the classroom. All members of the groups--by educational attainment, by years of experience, and by size of college--responded as definitely confident of their skills in the clinical setting.

Analysis

In this study, the comfort level and confidence of a new nursing faculty member in the clinical environment, as well as the process of evaluating students in the clinical setting, supports the work of Nugent et al. (1999) who found that new nurse educators scored the highest in the clinical skill domain on the Self-Efficiency Toward Teaching 
Inventory (SETTI), a 48-item questionnaire measuring self-confidence in the ability to perform specific teaching behaviors. Nugent et al. propose a mentoring plan and the assistance of experienced faculty to assist the new faculty member to develop confidence in the other areas of the teaching role.

6.c. Preparation of lecture content?

IS32. In the early days relate your knowledge of preparation of lecture content. How confident were you?

Only two faculty reported being somewhat confident in their ability to prepare a lecture and to present it. Across all groups, faculty reported that they were not confident in preparing and presenting the content. Support from mentors provided valuable assistance; however, one faculty member who did not have a mentor was persistent in trying until she was successful. "The more you teach the better off you are." Analysis

The findings in this qualitative examination of 19 nurse educators in ADN programs in Kentucky are consistent with the work from the AACN (2003). That study asked faculty members to rate 21 skills and characteristics that new faculty should have, two of which are important here: the ability to lead classroom discussions, and to develop competence as a lecturer. The ability to develop a presentation, using active learning strategies, and maintain student interest is a continual challenge. This result is consistent in the larger literature. Gray and Madson (2007) discussed the challenge of faculty to engage students in lecture and the techniques for its success.

\section{6.d. Examination development?}

IS33. In the early days of your teaching position relate your knowledge of development of examinations. How confident were you?

Faculty members in the study reported that they had little knowledge of 
examination development when they first assumed a teaching position. Even faculty in the most experienced group reported struggling to develop tests to measure student knowledge effectively. Most faculty stated examination preparation is an ongoing process even as experienced faculty. New and seasoned faculty reported attending professional development workshops for the newest testing options.

Analysis

The lack of confidence in examination preparation from this qualitative study is consistent with results from Nugent et al. (1999). Faculty had little confidence in the preparation of examinations and evaluation when asked to rate their confidence level across four domains: course preparation, instructor behavior, evaluation, and clinical teaching.

6.e. Curriculum revision and design?

IS34. In the early days of your teaching position, relate your knowledge of curriculum revision and design. How confident were you?

Interviewed faculty had little opportunity to assist in the revision of curriculum.

Of the 19 faculty only five had been part of a committee. Some faculty had taken curriculum design courses in their Master's work. One faculty member had chaired a curriculum revision committee and viewed it as a favorable experience. Even though the faculty in the "11+" group had the most nursing faculty experience, only two had the process in coursework as part of their advanced degree. No one had been involved with the curriculum design process.

Analysis

Penn, Wilson, and Rosseter (2008) outlined essential skills for nurse educators as a guide for nurses in the clinical setting interested in making the transition from nursing 
practice to a teaching role. Curriculum and course development includes the placement of content into a course, as well as locating a course into the larger curriculum. Both levels include the development of objectives and learning outcomes for students. Penn et al. noted the skills can be developed as part of the clinical knowledge of the nurse in clinical practice. With so few faculty having an opportunity to participate, perhaps this could be introduced to the new nurse educator in phases beginning first with syllabi revision. Curriculum re-design could then be considered at a later date.

6.f. Technology?

IS35. In the early days of your teaching position, relate your ability in the use of technology in the educational setting. How comfortable were you? How did that differ from your use of technology in the clinical setting?

Fifteen of 19 faculty reported they were comfortable with technology. They were using it on a daily basis. Some considered themselves advanced technology users. Some examples include podcasts, patient simulation, and student response systems. Several faculty agreed that their comfort level in the clinical setting made it easier, yet the technology used in some cases was more advanced in the educational setting. Other faculty who had been teaching over 11 years discussed their transition over the years from the transparency to the PowerPoint. Analysis

This study is consistent with the work of Penn et al. (2008) who described the importance for new faculty to have a good comfort level with technology. Teaching today's students requires an understanding of the best methods to get the message across and to include the learner in the action. The comfort level of the newer faculty in the use of technology can assist the more senior faculty in the adaptation of newer methods into their teaching. Penn et al. (2008) described the use of technology as a "powerful 
incentive" (p. 12) for teaching today's students. Developing techniques that engage students across the lifespan is essential.

6.g. Organization and time management?

IS36. Compare your ability to organize and manage your time in your teaching position with your position in the clinical area. How does it differ?

Three different responses came from the faculty with no particular difference based upon group membership for educational attainment, years of experience, or size of college. For some faculty, teaching required the most organization. One of the younger faculty members verbalized a frustration in not being able to complete all tasks within the work hours. Student interruptions and concerns took several hours from her day. She concluded that the clinical setting required the least amount of organization. Other faculty noted that the clinical setting was the most unpredictable and took the most physical energy, while teaching required the most organization. Some reported that they were organized no matter the setting.

Analysis

In a qualitative study, LaRocco and Bruns (2006) identified three dimensions of an organizing framework for new faculty: entry into academia and work life, participants' understanding of their university community, and perceptions of their relationships with their colleagues and their administrators. During interviews, faculty related difficulty in balancing their home life and work life. Some members of LaRocco and Bruns' study did have a sense of independence and autonomy; others voiced frustration and had difficulty in functioning independently. Support from other faculty was valuable while little support came from administrators.

The responses from the nurse educators in this study echoed these concerns, 
specifically on the pressures of workloads and the need for time management skills. Many were receiving support from the program coordinator and fellow faculty; these internal supports seemed to be especially important for new faculty as they developed skill sets appropriate to the less structured work environments of higher education. This issue had been noted often in the literature as nursing faculty as newcomers transition from the quite different milieu of clinical hospital to academe (Penn et al., 2008).

IS37. What is your sense of the collective cost/benefit of these barriers compared to clinical nursing? Has that influenced your decision to remain a nurse educator?

The issue of salary and benefits provided a good point of discussion for all members during the interviews. Many of the faculty were very adamant that money is important but the benefits of seeing student success and having flexibility in the job was just as important. The more altruistic side of the faculty member seems to prevail when it is money versus benefits. All faculty across the subcategories for educational attainment, experience, and size of program were concerned about the salary situation. As one faculty member noted, "Teaching has become a part of me. I enjoy what you get from teaching and watching students grow." Another faculty member said, "If the goal was to make more money, I would not be here."

In the Kentucky Community college system a pay raise had not been awarded for three years and faculty were concerned that another year would be the same. The high degree of salience of this factor was obvious. The exact wording of IS37 did not explicitly mention salary yet this interpretation was almost universal for these 19 respondents from ADN programs. Analysis

Fifty-nine percent of nursing faculty responded to the NLN (2003) national 
survey on job satisfaction that the salary would be the main reason for leaving the nursing faculty role. NLN noted that the need was there to advocate for higher salaries and salaries commensurate with workload. In the Garbee and Killacky (2008) study low pay was a source of dissatisfaction with $13.5 \%$ of all nursing faculty responding to the questionnaire. In that study, among all dissatisfaction themes, low pay was third in the ranking with time demands rated number one overall.

Penn et al. (2008) noted that nurses in clinical practice who make the decision to teach should expect potentially lower salaries especially if the appointment is for 9 or 10 months. In the Froman (1996) study to determine factors for attracting and accepting a nursing position, salary was the second of the top rated items for stimulating interest in a nursing faculty position. This was also a key factor in job satisfaction; the higher the salary, the greater the job satisfaction (Moody, 1996).

Brady (2007) noted that salary affects the recruitment of master's prepared faculty. It was proposed that the salary comparison of the nurse educator and the clinical practice nurse should not be made by comparing the salary of the staff nurse, but the nurse manager's salary where additional education is required.

Staurovsky (1992) studied levels of burnout and job satisfaction. In her study, the subscale, Present Pay, demonstrated low satisfaction with the job. Furthermore, of those nursing faculty who were termed "terminal burnout," a major concern from $60 \%$ of the study group who reported was low satisfaction with pay.

\section{Research Question 7}

What are the primary satisfactions of working as a nurse educator?

IS38. How satisfied are you with your job and performance? Why and in what ways? 
The greatest majority of nursing faculty responded in their interview they were satisfied to very satisfied with their job and performance. The group with the least experience reported they were very satisfied with their performance. One faculty member noted that she was never satisfied with her performance and always looked for ways to improve. Across all groups faculty were satisfied with their job and performance. Analysis

The findings in this qualitative examination of 19 associate degree nurse educators is supported by the NLN (2005) study, that job satisfaction among nurse educators is the highest among all nursing fields in general. Two-thirds of all nurse educators surveyed would choose nursing education again, but only half would choose to work in the same college or school.

Johnson (2001) studied nursing job satisfaction and organizational culture. Faculty job satisfaction was determined to be a contributor to faculty empowerment, and having a new administrator and faculty turnover was a statistically significant negative contributor to job satisfaction. This also supports a study by Moody (1996) on job satisfaction and its relationship to demographic variables, organizational characteristics, and role orientation. Faculty were found to be most satisfied with the work itself.

IS39. In the last year how many times have you received feedback for your performance? In what areas and why? To what extent is this influential in your career as a faculty member?

Faculty reported they had received positive feedback from their performance evaluations, from student evaluations, and from student success on the licensing exam as well as in clinical area as a graduate nurse. One faculty member in " $11+$ " group of the most experienced faculty received negative feedback from non-nurse division chair at performance evaluation at end of academic year (which was devastating). 
Analysis

In answering this question faculty included who gave the feedback. Most of the faculty noted that students gave them daily feedback. Moody (1996) looked at literature to verify the responses on who gives feedback, not necessarily in what form. Moody found that job satisfaction had an inverse relationship with years at current institution and satisfaction with opportunities for promotion. This is also supported by the NLN (2003) study. To enhance the job satisfaction of the faculty member with tenure, Moody suggested expanding the promotion system or the way to recognize accomplishments of the more senior faculty.

IS40. Who gave you that feedback? In what areas and why? Does the person who is giving positive feedback influence your satisfaction as a nursing faculty member? In what way?

From the 19 interviews, the nursing coordinator was a key person in providing feedback. Fellow faculty, the Division Chair or Dean and the students were important as well. Faculty agreed that it did influence their satisfaction as a faculty member. One faculty member received negative feedback from her non-nurse Division Chair. Despite what the faculty member thought was an exceptional year performance-wise, the supervisor did not agree. As a result the faculty member is looking for another job despite being a senior faculty member with more than 11 years of teaching experience. Analysis

In the NLN (2005) study $73 \%$ of the nurse educators surveyed, responded that they received constructive feedback and guidance from fellow faculty members. But they did not receive as much feedback as desired from administrators. Also, $85 \%$ of the nurse educators surveyed believed that their supervisor (Division Chair, Dean, Program Coordinator) was supportive of their efforts to continually improve their teaching, $77 \%$ 
indicated support of service activities, and $74 \%$ were supportive of their efforts to improve their scholarship. Overall the 19 respondents related that besides from fellow faculty they received support from administrators. In fact one nurse educator responded that she had not received positive feedback from the division chair and was considering leaving. One respondent noted that her program administrator had provided additional opportunities for faculty development.

IS41. What is the nature of the positive feedback you receive? Formal recognition? Informal encouragement/praise? Concrete (as in course release, raise, other)? Psychic (as in good for the self and soul)?

Faculty members reported they received more informal feedback than formal. Faculty like to hear "job well done." One faculty member may receive an anonymous piece of chocolate when she is especially stressed. To her that is powerful in its message. Several mentioned the combination of concrete and psychic feedback they receive and how comforting it is. Many report receiving more psychic feedback and informal encouragement which sustains them.

Analysis

Faculty agreed that formal rewards of raises and bonuses are exceptionally motivating. But there are other ways that faculty can receive the message that they are valued. The "little piece of chocolate" is the silent message that others care. Seeing graduate nurses in the clinical setting looking efficient and demonstrating wonderful caring skills to the patients, that too is a definite warm moment. In general the sentiments of these 19 nursing faculty provide powerful reinforcement of the classic work of Lortie (1975) who examined the work environment, culture, and identity of public school teachers. Quintessential to Lortie's study were psychic rewards of teaching--love of learning, the feedback from students, the relationships with their charges, and the 
satisfaction of seeing them learn and become successful. Although this research examined ADN programs, the parallels are uncanny with respect to the primacy of such psychic rewards for these nursing faculty.

IS42. How much do the different types of satisfaction influence your career choice as a nursing educator?

Raises and benefits were important to the group of faculty members, but also the feedback from students and fellow faculty members seemed to be very important. One faculty member commented, "I think you have to have all that to stay here." A new faculty member's response, "In a clinical position you do not get much feedback from managers in the clinical setting. I feel more valued here." Also, in response to the question of types of satisfaction and its influence, "It's [feedback from students] what keeps me here--for a better lack or words."

Analysis

The responses for this question essentially mirrored those from IS41. No further analysis is provided.

IS43. Do you have any final thoughts or feelings about being a nursing educator? To what extent have these influenced your satisfaction with your career choice?

Faculty members cited the enjoyment in working with students and seeing them grow from the first day of clinical experience to getting ready for graduation. The combination of teaching and learning constitutes the essence of what these faculty do. Typifying such confidence faculty members was this statement, "I Love being a faculty member, I really feel good about myself and have a sense of accomplishment." Analysis

This group of faculty embodied the role of the nurse educator, their enjoyment in 
working with students not only was heard but was seen on their faces as they smiled, became animated and spoke with the passion of their love for teaching and nursing. Lortie's (1975) work supports this passion and love for teaching. The development of the student is a major concern for the faculty in this study and is supported by Lortie's classic study of schoolteachers.

These nurse educators' love for learning was evidenced in their return to school to attain additional degrees as well as their continual search for the latest information to impart to their students. However, pride in knowing they had a part in the education of a caring and tremendously valuable graduate nurse.

\section{Recommendations}

This section is divided into two sections for review, one on policy and practice and one on future research. Policy and practice considerations provide an opportunity to identify discussion points for future actions.

\section{Policy and Practice Considerations}

First, develop a Nurse Educator Academy. The Academy could be organized on a state-wide basis and would be the first step in the orientation process of new nursing faculty members. Each college currently attempts to orient its new faculty members over a period of time in either a formal or informal manner. This orientation would be provided at a central location to which new faculty would come, network, and attend sessions taught by faculty who are identified as experts in the field. This orientation would assist new faculty in the transition from clinical practice to education. Topics covered during the session could include: teacher and college expectations, teaching load, student relationships and social networking, lecture preparation, the use of technology, exam preparation, technology (patient simulation), professional development 
opportunities, and possibly the work of the college--performance review, internal and external service, promotion and tenure. Because these vary at each college, the instruction would identify protocol and activities that would be basic to all faculty.

Second, develop a mentoring program for all new faculty. Currently, formal and informal mentoring programs vary by college. Some of the interviewed nurse educators had mentors while others did not. If one college had a particularly effective one, perhaps that could be shared for others to implement.

Third, review the practice of assigning student advising during the first year of teaching. By foregoing this activity for one year the nurse educator would develop the role of instructor within the classroom and clinical environment.

Fourth, review the practice of requiring new faculty to complete external service responsibilities the first year. From this study, external service was difficult for new faculty to understand and to complete. By providing additional time to complete teaching assignments, the new nurse educator would be less overwhelmed.

Fifth, work towards salary parity. Nursing faculty spoke of the difference in compensation when moving from the clinical setting to the college. Although the subjects interviewed responded with passion and love for nursing and teaching, below the surface seemed a frustration and to a certain extent, somewhat reconciliation that salaries in academe are not comparable to the clinical environment or other non-nursing faculty. The current status of parity of the two environments and within academe should be examined throughout the state nurse education system. A group of administrators and nurse educators could be convened to address the issue. Nursing faculty are usually on a 9month contract and have the opportunity to work in the clinical setting during the summer or in some areas, during vacations. However, as nurse educator responsibilities increase, 
the faculty member could be spending the summer or holiday periods working on lecture development of course outlines. Since not on contract, the nurse educator does not get paid during the summer months.

Sixth, address recruitment of new faculty with an additional marketing plan. This would include the benefits of teaching, flexibility of schedule, organizational climate, tuition advancement, and the prestige/status of the position. Particularly important would be advertising the rewards for faculty nurses of realizing their Love of Learning, Teaching and Nursing, or their opportunity to teach, to work with and see their students become successful nurses in their own right. Especially important in this regard would be testimonials from practicing nurse faculty on just how rewarding these personal accomplishments can be.

Seventh, focus on the "personal touch" in recruitment efforts. Several of these faculty indicated that they came to teaching because of the specific invitation, overture, or recruitment—-person to person—-from a nursing faculty member or administrator who knew of their work in the clinical setting.

Eighth, encourage the process of certification as a nurse educator through the National League for Nursing ( n.d.). This designation signifies the excellence of the specialty role of nurse educator. Certification includes knowledge development in all areas of nursing education (facilitate learning, development of evaluation and testing, scholarship of teaching, etc.). As the new faculty member moves through the process toward eligibility, the knowledge gained would be valuable in the teaching responsibilities and the overall development of a faculty member within the program as well as the college. 
Finally, identify students within the nursing program who are interested in teaching. Nursing programs should seek opportunities for scholarships or grants to assist those students to attain the appropriate education. Those students should be mentored and perhaps opportunities found for them to "student teach" in their program prior to graduation from the Master's program. This would provide a smoother transition to the faculty role once the new faculty member assumes a full time role.

\section{Future Research}

First, this study focused only on educators in associate degree nursing programs in Kentucky. Certainly, nurse educators in baccalaureate and practical nursing programs have their own story to tell. By interviewing nurse educators from the various levels and comparing their diversified responses, additional themes and ideas may be realized to provide an overall view of nurse educators in Kentucky.

Second, throughout the study, responses from the nurse educators provided additional concerns for the program coordinators and their thoughts pertaining to career change. By interviewing those nursing leaders, a different view of nursing education could be identified. This would provide an additional level of decision-making by nurses-from nursing to nursing faculty to the leadership role within the nursing program.

Third, the Astin's Sociopsychological Theory of Career Choice (1984) provides a framework for identifying factors in the selection of careers. Research with other health occupations would provide additional insights into the relevance of the theory. Originally the theory as developed, examined women and their career choice. Lindholm (2004) included men in her study to ascertain its overall generalizability. In this study, nursing is predominately female and only one male was included in the sample. Sampling more men in nursing or sampling another occupation that is more male dominated would add 
additional information to the applicability of the theory.

Fourth, faculty in the current study, who had a Master's degree prior to employment in academe, had completed the advanced degree to enhance their role within the clinical setting, rather than specifically for teaching in a nursing program. There may be other prospective nursing faculty who are interested in teaching, but are ambivalent about returning to obtain an additional degree. Further research on the clinical nurse, their potential interest in becoming nurse faculty, and barriers to their actual return to school would be important to recruitment as nursing faculty.

Fifth, the responses from the interview schedule provided initial insight into the role of the administrator and other faculty in recruitment of new nurse educators. That "personal touch" may provide an important lure for nurses in clinical practice or even in advanced degree programs to become full time nursing faculty. To the researcher's knowledge, however, this finding has not been previously reported. More work is needed to confirm this insight.

Sixth, nursing faculty discussed the role that social media has in the context of the student-instructor relationship. Faculty in the study responded that there is a limit as to who and what is shared on the social media sites. Literature dealing with the faculty perspective in sharing information with their nursing students and the access to social media sites was not found in the literature. This obviously new phenomenon is clearly deserving of more study.

Seventh, the decision for a nurse to change careers from clinical to teaching included the physical aspect of the job itself. Three of the group interviewed discussed the physically exhausting nature of their former job and the difficulty in continuing to work 12-18 hours during the day, with a short time frame between assigned days. One 
faculty member stated that her body could not take the physical nature of the position. Another likened the clinical work to creating a "Post traumatic stress" situation which she could not turn off mentally after working the 12-hour shifts. Research into the subject of the physically exhausting work of the clinical situation may be the opportunity to transition an excellent clinician into the teaching role.

Eighth, the nursing faculty in the study were not asked to provide their area of specialty during the interview nor on the background questionnaire. Programs of nursing hire faculty with experience in the areas of nursing in which they are teaching (psychiatric nursing, medical-surgical, pediatrics, and obstetrics). Future research into the present group of faculty and their area of specialty in which they practiced may provide additional information as to where recruitment should be focused.

Finally, several aspects of Dan Lortie's (1975) research were cited in this study. The immediacy of the short-term relationship and success of the students versus the longterm goal of resolving the nursing shortage parallels his work with public school teachers. The success of the student is a main theme in both this study and his work. It seems beyond coincidence that both Lortie's work and this current study were qualitative investigations which are particularly germane to understanding sentimentality and other psychic/identity phenomena. More research into the focus of success of students and the involvement of the nurse educator would be of benefit to job satisfaction as well as student retention.

\section{Conclusions}

This qualitative study provided insights into the thoughts and feelings of nursing faculty in associate degree nursing programs in Kentucky. The members shared their passion, their concerns, and their thoughts for the future. For some, a question brought a 
teary response, or gave them a pause to think. The responses from the interview gave the researcher a snapshot of the nurse educators for that moment. Generalizability of the results of the study rests in the collection of the data from the two instruments and the verification of the information in the transcripts verified by the subjects.

Nineteen nursing faculty participated in the study. Each completed a Background Questionnaire for Nurse Educators and the Nurse Educator Interview Schedule. By tape recording their responses to open-ended questions, the subjects responded with their beliefs about their profession, what made their job better, and what gave them the "warm and fuzzy moments" that sustained them from day to day. Overall, the key factors that affected their decision to teach were flexibility of their environment, their love for teaching, working with students, and the overall pride in knowing they had a part in the education of a caring and tremendously valuable graduate nurse. Faculty compensation was an underlying theme that should be addressed after gathering additional information from other faculty.

Faculty responses to the question concerning collegiality and the comparison from clinical to academic atmospheres seems to be dependent more on the unique groups to which each nurse belongs. The nurse educators talk of the relationships, although different in nature, centered in the clinical setting on the care of the patient. In the college setting the emphasis was on the education of the student. But in the clinical setting, some nurse educators discussed the lack of opportunity to socialize as a group. The college setting provides that opportunity for the socialization and the development of the team relationship. For the majority of nurse educators, this was true. There were a few faculty who believed they would fit into any setting clinical or academe and receive satisfaction from whatever group they were in. 
The results of this study support other studies dealing with career choice, work environment, pedagogical considerations, expectations of the faculty role, primary barriers and primary satisfiers among nurse educators. The data suggest that the nineteen nurse educators were diverse in relation to educational attainment, years of nursing faculty experience, and size of college in which program was located. But many times their responses to the Background Questionnaire for Nurse Educators and the Nurse Educator Interview Schedule came together as one voice. Their responses to the questions provided a rich description of what their thoughts and feelings were in relation to the questions asked.

The data lend additional support for the Sociopsychological Model of Career Choice by Helen Astin. The model includes four constructs: (a) motivation, (b) expectations, (c) sex-role socialization, and (d) structure of opportunity. In listening to the nurse educators' responses to the Nurse Educator Interview Schedule that were related to pursuing a career in nursing and to becoming a nurse educator, the categories of career choice and motivation are representative of the theoretical framework. To the question concerning the choice of nursing as a profession, there were three reasons for their choice: (a) early family experiences (relative in nursing or health care), (b) situation (ill family member), or (c) caring for people. As for sex-roles and opportunity structures, nursing has been and remains a female dominated profession. There were two faculty in the study who chose nursing over other interests because the career as a woman doctor or a lawyer at the time was frowned upon by society and primarily men were able to enter those professions. While those days are hopefully past, the "caring" nature of nursing and the low salary compared to clinical practice and even some other fields in academe provide a current environmental milieu in which gender-roles are just as strong as they 
were forty years ago.

The choice to go into nursing education was based upon specific situation (working at the hospital and having a challenging day, a faculty member who guided them or physical stress that made clinical work no longer an option). According to Astin (1984), the desire to work fulfills the survival needs. The pleasure needs are derived from the satisfaction the participant receives when working. Contribution needs are met by assisting others to the attain well-being. Faculty members told of their love for nursing and desire to help others.

Lindholm (2004) addressed Astin's (1984) model and defined it from an educator's perspective. Lindholm visualized the role of an educator as being autonomous. She characterized the flexibility that nurse educators valued in their decision to change careers from clinical practice to education. When Lindholm interviewed the subjects in her study, she described the group as being passionate and animated during their responses. "There was also a uniformly expressed sense of appreciation for what many referred to as the 'privilege' of having tremendous freedom to engage not only in a variety of pursuits but in the intellectual pursuits about which they are most passionate" (p. 612).

One fourth of those Lindholm interviewed spoke of the nature of their work as a "true calling." This researcher experienced the same passion to the responses in the present study. Clearly these nursing faculty love teaching and derive extensive psychic rewards from working with students.

The challenge for these nurse educators is to channel their love of teaching-Lortie's (1975) psychic rewards, predominately through success with individual students-into professional excellence wherever essentially all students are helped to become 
professional nurses. That will require better understanding of the processes by which clinical nurses become outstanding nursing faculty as well as better insights into how to change the reward structures that encapsulate nurse educators. With progress on both fronts, there may be hope that sufficient numbers of nursing faculty can be recruited to address the looming crisis in nursing care for an aging population. 


\section{REFERENCES}

Adams, P. F. (1985). Holland's theory of careers applied to nursing faculty teaching in public community and universities in Kentucky. Dissertation Abstracts International, 46(03), 622.

American Association of Colleges of Nursing. (2003). Faculty shortages in baccalaureate and graduate nursing programs: Scope of the problem and strategies for expanding the supply. Washington, DC: Author

American Association of Colleges of Nursing. (2009). Nursing shortage fact sheet. Washington, DC: Author. Retrieved February 7, 2010, from http:/www.aacn.nche.edu/media/shortageresource.htm

Anfara, V. A., Brown, K. M., \& Mangione, T. L. (2002). Qualitative analysis on stage: Making the research process more public. Educational Researcher, 31(7), 28-38.

Ashton, K. C. (1998). A replication of experienced graduate nurse faculty orientation offerings and needs. Journal of Nursing Education, 37, 295-301

Ashton, P. (1985). Motivation and the teacher's sense of efficacy. In C. Ames \& R. E. Ames (Eds.), Research on motivation in education. Volume 2: The classroom milieu (pp. 141-171). Orlando, FL: Academic Press.

Astin, H. S. (1984). The meaning of work in women's lives: A sociopsychological model of career choice and work behavior. The Counseling Psychologist, 12(4), 117126.

Bandura, A. (1994). Self efficacy. In V.S. Romachaudren (Ed.), Encyclopedia of human behavior (Vol. 4, pp. 71-81). New York: Academic Press.

Bauerhaus, P. I., Staiger, D. O., \& Auerbach, D. I. (2000). Implications of an aging registered nurse workforce. Journal of the American Medical Association, 283, 2948-2954.

Berger, K. J., \& Williams, M. B. (1999). Fundamentals of nursing: Collaborating for optimal health (2nd ed., Vol. 1). Stamford, CT: Appleton Lange.

Berry, L. H., Hammons, J. O., \& Denny, G. S. (2001). Faculty retirement turnover in a community colleges: A real or imagined problem? Community College Journal of Research \& Practice, 25, 124-137. 
Bonjean, C. M., Brown, B. J., Grandjean, B. D., \& Macken, P. O. (1982). Increasing work satisfaction through organizational change: A longitudinal study of nursing educators. Journal of Applied Behavioral Science, 18, 357-369.

Boughn, S. (1999). Why do women choose nursing? Journal of Nursing Education, 38, 156-161.

Brady, M. S. (2007). Recruitment and retention of associate degree nursing faculty. Journal of Nursing Education, 46, 190-192.

Brendtro, M., \& Hegge, M. (2000). Nursing faculty: One generation from extinction? Journal of Professional Nursing, 16, 97-103.

Bullough, V. L., \& Bullough, B. (1969). The emergence of modern nursing (2nd ed.). Toronto, Canada: Macmillan Company.

Burns, N. \& Grove, S. K. (2005). The practice of nursing research: Conduct, critique, and utilization (5th ed.). St. Louis: Elsevier.

Carnegie Foundation for the Advancement of Teaching. (n.d.). Classification of institutions of higher education. (n.d.). Retrieved April 10, 2010, from http://classifications.carnegiefoundation.org/

Center for the Health Professions, The, University of California. (2002). Critical challenges: Revitalizing the health professions for the twenty-first century. Retrieved July 29, 2002, from http://www.futurehealth.ucsf.edu/summaries/challenges.html

Choudhry, U. K., (1992). New nursing faculty: Core competencies for role development. Journal of Nursing Education, 31, 265-272.

Craine, D. M. (2000). Nursing faculty shortage in Georgia: An impending crisis. Georgia Nursing, 60(3), 12-15.

Creswell, J. W. (1998). Qualitative inquiry and research design: Choosing among five traditions. Thousand Oaks, CA: Sage Publications.

Danquah, F., \& Miller, S. K. (2007). Cocoa farming in Ghana: Emic experience, etic interpretation. Southern Rural Sociology, 22(1), 65-79. Retrieved February, 3, 2010, from

http://www.ag.auburn.edu/auxiliary/srsa/pages/Articles/SRS\%202007\%2022\%20 $1 \% 2015-27 . p d f$

Deloughery, G. (1977). History and trends of professional nursing (8th ed.). St. Louis: C. V. Mosby. 
Dolan, J. A. (1968). History of nursing (12th ed.). Philadelphia: W. B. Saunders.

Edelstein, J. A. (2000). Burnout and job satisfaction of full-time nurse educators between 39-56 years of age in state supported baccalaureate programs in Wisconsin. (Doctoral dissertation, University of Arkansas, 2000). Dissertation Abstracts International 61(09), 4638. (UMI No. 9987299)

Fain, J. A. (1987). Perceived role conflict, role ambiguity, and job satisfaction among nurse educators. Journal of Nursing Education, 26, 233-238.

Feeg, V. D. (2004). Doctoral education: Why now and why you? Pediatric Nursing, $30(1), 8$.

Froman, R. (1996). Attracting an expert, or what nurse leaders look for in academic jobs. Nursing Outlook, 44, 239-242.

Fugate, A. L., \& Amey, M. J. (2000). Career stages of community college faculty: A qualitative analysis of their career paths, roles, and development. Community College Review, 28(1), 1-23.

Gall, D. G., Borg, W. R., \& Gall, J. P. (1996). Educational research: An introduction (6th ed.). New York: Longman Publishers.

Garbee, D. D., \& Killacky, J (2008). Factors influencing intent to stay in academia for nursing faculty in the southern United States of America. International Journal of Nursing Education Scholarship. 5(1), 1-15. Retrieved October 17, 2009, from http://www.bepress.com/ijines/vol5/iss1/art9

Genrich, S. J., \& Pappas, A. (1997). Retooling faculty orientation. Journal of Professional Nursing, 13, 84-89.

Grandjean, B. D., Aiken, L. H., \& Bonjean, C. M. (1976). Professional autonomy and the work satisfaction of nursing educators. Nursing Research, 25, 216-221.

Gray, T., \& Madsen, L. (2007). Ten easy ways to engage your students. College Teaching,55,83-87.

Hart, J. F. (1995). "Rural" and "farm" no longer the same. In E. N. Castle (Ed), The changing American countryside: Rural people and places (pp. 63-76). Lawrence, KS: University of Kansas.

Hermann, M. M. (1997). The relationship between graduate preparation and clinical teaching in nursing. Journal of Nursing Education, 36, 317-322.

Higgins, C. S., \& Hawthorne, E. M. (1994). The successful community college instructor: A profile for recruitment. Community College Review, 21(4), 27-37. 
Hinshaw, A. (2001). A continuing challenge: The shortage of educationally prepared nursing faculty. Online Journal of Nursing Issues in Nursing, 6, 1-14.

Hofstadter, R. (1994). Social Darwinism in American thought. Boston: Beacon Press.

Holder, V. L. (2003). From hand maiden to right hand: The birth of nursing in America. Association of Operating Room Nurses AORN Journal, 78, 618-632.

Johnson, B. A. (2001). Organizational culture and job satisfaction as antecedents for empowerment of associate degree nursing faculty (Doctoral dissertation, Georgia State University, 2001). Dissertation Abstracts International, 62, 162.

Joint Commission on Accreditation of Healthcare Organizations. (2002). Health care at the crossroads: Strategies for addressing the evolving nursing crisis. Oakbrook Terrace, IL: Author.

Kalisch, B. J., \& Kalisch, P. A. (2004). American nursing: A history (4th ed.). Philadelphia: Lippincott Williams and Wilkins.

Kavoosi, M. C., Elman, N. S., \& Mauch, J. E. (1995). Faculty mentoring and administrative support in schools of nursing. Journal of Nursing Education, 34, 419-426.

Kentucky Board of Nursing. (n.d.). Summary of pre-licensure RN programs. Retrieved April 26, 2010, from http://kbn.ky.gov/NR/rdonlyres/8A116559-049D-44AF9C5A-D10EEBB34476/0/rn_summary.pdf

Kentucky Board of Nursing. (n.d.). 201 KAR 20:310. Faculty for prelicensure registered nurse and practical nurse programs. Retrieved November 17, 2010, from http:// www.lrc.state.us/kar/201/020/310.html

Kennerly, S. M. (1989). Leadership behavior and organizational characteristics: Implications for faculty satisfaction. Journal of Nursing Education, 28, 198-202.

Kozier, B., Erb, G., Berman, A., \& Snyder, S. (2004). Fundamentals of nursing, concepts, process, and practice (7th ed.). Upper Saddle River, NJ: Pearson Prentice Hall.

LaRocco, D., \& Bruns, D. (2006). Practitioner to professor: An examination of second career academics' entry into academia. Education, 26, 626-639.

Lia-Hoagberg, B. L. (1982). Professional activities and personality characteristics of nursing faculty compared with those of other academic women (Doctoral dissertation. University of Minnesota, 1982). Dissertation Abstracts International, 51,1060 . 
Lindholm, J. A. (2004). Pathways to the professoriate: The role of self, others, and the environment in shaping academic career aspirations. Journal of Higher Education, 75, (6), 603-635.

Lortie, D. C. (1975). Schoolteacher: A sociological study. Chicago: University of Chicago Press.

Lubbert, V. M. (1995). Structure and faculty perception of climate in schools of nursing. Western Journal of Nursing Research, 17, 317-328.

Macnee, C. L., \& McCabe, S. (2008). Understanding nursing research (2nd ed.). Philadelphia: Lippincott Williams \& Wilkins.

McMurray, A. J. \& Sorrells, D. (2009). Bridging the gap: Reaching first-generation students in the classroom. Journal of Instructional Psychology, 36(3), 2-6.

Meadus, R. J. (2000). Men in nursing: Barriers to recruitment. Nursing Forum, 35(3), 513.

Merriam, S. B. (2002). Qualitative research in practice: Examples for discussion and analysis. San Francisco: Josey-Bass Publishers.

Miles, M. B., \& Huberman, M. (1994). Qualitative data analysis: An expanded sourcebook (2nd ed.). Beverly Hills, CA: Sage Publications.

Miller, S. K., with Jack, Z. M. (2007). Storytelling as narrativity: Rural life through the prism of social tensions. Southern Rural Sociology, 22(1), 15-27.

Milosheff, R. J. (1990). Factors contributing to job satisfaction at the community college. Community College Review, 18(1), 12-23.

Moody, N. B. (1996). Nurse faculty job satisfaction: A national survey. Journal of Professional Nursing, 12, 277-288.

Morin, K. H., \& Ashton, K. C. (1998). A replication study of experienced graduate nurse faculty orientation offerings and needs. Journal of Nursing Education, 37, 295301.

National Center for Educational Statistics. (n.d.). Integrated postsecondary education data system. Retrieved April 26, 2010, from http://nces.ed.gov/ipeds/

National League for Nursing. (2005). A national study of faculty role satisfaction 2003. New York: Author.

National League for Nursing. (2006). Nurse educators 2006: A report of the faculty census survey of $R N$ and graduate programs. New York: Author. 
National League for Nursing. (n. d.). Certification for nurse educators. Retrieved December 2, 2010, from http://www.nln.org.facultycertification/ prepare/index.htm

NLN Nursing Education Policy Newsletter. (2010, May). National League for Nursing, 7(3). Retrieved May 14, 2010, from_http://www.nln.org/governmentalaffairs/ newsletter/vol17_issue $3 . \mathrm{htm}$

Nugent, K. E., Bradshaw, M. K., \& Kito, N. (1999). Teacher self-efficacy in new nurse educators. Journal of Professional Nursing, 15, 229-237.

Oermann, M. H. (1998). Role strain of clinical nursing faculty. Journal of Professional Nursing, 14, 329-334.

Okrainec, G. D. (1994). Perceptions of nursing education held by male nursing students. Western Journal of Nursing Research, 16, 94-107.

Penn, B. K., Wilson, L., \& Rosseter, R. (2008). Transitioning from nursing practice to a teaching role. Online Journal of Issues in Nursing, 13(3), 9-31.

Polit, D. F., \& Hungler, B. P. (1983) Nursing research: Principles and methods. Philadelphia: J. B. Lippincott.

Piscopo, B. (1994) Organizational climate, communication, and role strain in clinical nursing faculty. Journal of Professional Nursing, 10, 113-119.

Ryan, M., \& Irvine, P. (1994). Nursing professorate in America: Projected retirements and replacements. Journal of Nursing Education, 33, 67-73.

Siler, B. B., \& Kleiner, C. (2001). Novice faculty: Encountering expectations in academia. Journal of Nursing Education, 40, 397-403.

Southern Regional Education Board. (2000). SREB study indicates serious shortage of nursing faculty. Washington, DC: U.S. Government Printing Office.

Staurovsky, L. G. (1992). Burnout in nurse faculty: Relationship to job satisfaction and self identified job related stressors. Unpublished doctoral dissertation, University of Texas, Austin.

Steele, R. (1991). Attitudes about faculty practice, perceptions of role, and role strain. Journal of Nursing Education, 30, 15-22.

Stewart, I. M., \& Austin, A. L. (1962). A history of nursing. New York: Putnam.

Talbot, L. A. (2000). Burnout and humor usage among community college nursing faculty members. Community College Journal of Research \& Practice, 24, 359374. 
United States Department of Health and Human Services. (2000). The registered nurse population. Washington, DC: U.S. Government Printing Office.

Vierra, A., Pollack, J., \& Golez, F. (1998). Reading educational research (3rd ed.). Upper Saddle River, NJ: Prentice-Hall, Inc.

Watt, H., \& Richardson, P. (2007). Motivational factors influencing teaching as a career choice: Development and validation of the FIT-Choice Scale. The Journal of Experimental Education, 75, 167-202.

Winter, P. A. (1996). The application of marketing theory to community college faculty recruitment: An empirical test. Community College Review, 24(7), 3-16.

Winter, P. A., \& Kjorlien, C. L. (2000a). Community college faculty recruitment: Predictors of applicant attraction to faculty positions. Community College Review, 28(1), 23-40.

Winter, P. A., \& Kjorlien, C. L. (2000b). Community college faculty recruitment: Effects of job mobility, recruiter similarity-dissimilarity, and applicant gender. Community College Journal of Research \& Practice, 24, 547-567.

Winter, P. A., \& Munoz, M. A. (2001). Community college business faculty recruitment: Association between personal characteristics and applicant's rating of the job. Community College Journal of Research and Practice, 25, 639-651.

Witney, L. W. (1990). Nurse educators: Self concept and leadership behaviors. (Doctoral dissertation, Illinois State University, 1990). (UMI No. PUZ9101129)

Wolfertz, J. R. (1999). The effects of institutional climate on the recruitment and retention of part-time associate degree nursing faculty. Dissertation Abstracts International, 60(07), 2347. (UMI No. 9939567) 
APPENDICES 
APPENDIX A

BACKGROUND QUESTIONNAIRE FOR NURSE EDUCATORS

AND NURSE EDUCATOR INTERVIEW SCHEDULE

MAPPED TO RESEARCH QUESTIONS 
Appendix A

Background Questionnaire For Nurse Educators and

Nurse Educator Interview Schedule Mapped to Research Questions

Research questions are shown in bold. BQ is short for Background Questionnaire for Nurse Educators; IS represents Nurse Educator Interview Schedule.

\section{Research Question 1}

To what extent are personal and demographic factors influential:

a. Age?

b. Marital status?

c. Children or other family responsibilities?

d. Years of nursing experience?

e. Years of nursing faculty experience?

f. Family educational attainment?

g. Educational attainment?

BQ1 Gender

BQ2 Location-Small or Medium/Large

BQ3 Age

BQ4 Marital Status

BQ5 Children and other family responsibilities

BQ6 Years of nursing experience

BQ 7 Years of nursing faculty experience

BQ8 Mother's educational attainment

Less than high school 
High School

Some college

College

Professional or advanced degree

BQ9 Father's educational attainment

Less than high school

High School

Some college

College

Professional or advanced degree

BQ10 My educational attainment

Less than Masters

Working on Masters

Masters Degree

Working on Doctorate

Doctoral Degree

\section{h. Influential person or event?}

IS1 Tell me when and why you decided on nursing as a profession?

IS2 What influenced your decision to become a faculty member?

IS3 Was there a key person/situation that supported your decision to teach?

In what way?

i. College Classification--Small or Medium/Large?

Selected by researcher from institutional criteria

\section{j. Other?}


IS4 Anything else you would like to add about personal or background factors that influenced your decision to teach?

\section{Research Question 2}

In what ways are educational degree requirements for faculty positions:

a. Influence decisions to become a faculty member?

IS 5 What was your original educational level for licensure? Did this influence in any way your eventual decision to teach? How and why?

IS 6 What was your original educational level when you started teaching? Did this enter into your decision to teach? How and why?

\section{b. Influence career trajectory (tenure and promotion) and retention?}

IS 7 What are your college's requirements for terminal degrees for promotion and/or tenure? To what extent did this influence your decision to teach?

\section{Research Question 3}

To what extent is the work environment in academia (compared to clinical nursing) influential:

\section{a. Flexibility?}

IS8 Can you compare the flexibility of your teaching position within the college setting and your previous clinical position? Do you consider this a benefit or a burden of teaching? Why?

IS9 What is your sense of this flexibility now (as an experienced faculty member) compared to when you started? What was your perception of this at the time when you first started teaching? Was it influential in your decision to change your career? Why? 


\section{b. Collegiality?}

IS10 Can you compare the collegiality of your teaching position within the college setting and your previous clinical position? Do you consider this a benefit or a burden of teaching? Why?

IS11 What is your sense of this collegiality now (as an experienced faculty member) compared to when you started? What was your perception of this at the time when you first started teaching? Was this influential in your decision to change your career? Why?

\section{c. Organizational climate?}

IS 12 Compare the organizational climate of your teaching position within the college setting and your previous clinical position? Do you consider this a benefit or a burden of teaching? Why?

IS13 What is your sense of this organizational climate now (as an experienced faculty member) compared to when you started? What was your perception of this at the time when you first started teaching? Was it influential in your decision to change your career? Why?

\section{d. Prestige/status?}

IS14 Can you compare the prestige/status of your teaching position within the college setting and your previous clinical position? Do you consider this a benefit or a burden of teaching? Was it influential in your decision to change your career?

IS15 What is your sense of this prestige/status now (as an experienced faculty member) compared to when you started? What was your perception of this at the time when you first started teaching? Was it influential in 
your decision to change your career? Why?

e. Other?

IS16 Can you think of any other factors related to work environment that influenced your decision to change from clinical nursing to faculty status? How and why?

\section{Research Question 4}

To what extent are pedagogical considerations influential:

\section{a. Love of teaching?}

IS17 Think about your reasons for changing from the clinical environment to teaching. How do you feel when you teach?

IS18 When you started your career as a nursing faculty member, did you see teaching as a plus or a minus? Why?

\section{b. Lifelong learning?}

IS19 Talk about the extent that you have always loved or disliked school. Did this have any influence on your decision to enter teaching? In what ways?

\section{c. Possibility of influencing the next generation of nurses?}

IS20 How would you describe the rewards of teaching? Has your sense of this changed since you started this career? In what ways?

IS21 How do you feel about your relationship with your student nurses?

IS22 To what extent are these feelings connected to the immediacy of the classroom? To long term issues like the nursing shortage? Other factors?

\section{d. Content knowledge and skills?}


IS23 How do you view the intellectual challenges of staying current with the knowledge about your field? Did this have any influence on your decision to enter teaching? In what ways?

\section{e. Other?}

IS24 Can you think of any other factors related to pedagogical considerations that influenced your decision to change from a clinical nurse to a faculty member?

IS25 Which of these would be the most important factor in your decision to make the change? Why?

\section{Research Question 5}

What are the expectations for "being nursing faculty" when first hired regarding:

\section{a. Teaching load?}

IS26 Think back to the first days of your teaching job. What were the expectations with respect to teaching load? Did this influence your decision to enter higher education? In what ways?

b. Service?

IS27 In relation to internal and external service to the college, how did your expectations and those of your supervisor differ? How were they similar?

\section{c. Scholarly writing and research?}

IS28 Early in your career, what were the expectations for scholarly writing and research? How did that differ from those of your supervisor? 


\section{d. Earning tenure and/or promotion?}

IS29 How much of a concern were the requirements for earning tenure and promotion when you decided to become a faculty member? In what ways?

\section{Research Question 6}

What are the primary barriers that you experienced in becoming a nurse educator?

\section{a. Student evaluation?}

IS30 Thinking back to your early days as a nurse educator, relate your knowledge of student evaluation. How confident were you? Did this enter into your decision to enter teaching?

\section{b. Clinical evaluation of students?}

IS31 In the early days as a nurse educator, relate your knowledge of clinical evaluation of students. How confident were you? Did this affect your decision to change professions?

c. Preparation of lecture content?

IS32 In the early days relate your knowledge of preparation of lecture content. How confident were you?

d. Examination development?

IS33 In the early days of your teaching position, relate your knowledge of development of examinations. How confident were you?

\section{e. Curriculum revision and design?}

IS34 In the early days of your teaching position, relate your knowledge of curriculum revision and design. How confident were you? 


\section{f. Technology?}

IS35 In the early days of your teaching position, relate your ability in the use of technology in your education setting. How comfortable were you? How did that differ from your use of technology in the clinical setting?

\section{g. Organization and time management?}

IS36 Compare your ability to organize and manage your time in your teaching position with your position in the clinical area. How does it differ?

IS37 What is your sense of the collective cost/benefit of these barriers compared to clinical nursing? Has that influenced your decision to remain a nurse educator?

\section{Research Question 7}

\section{What are the primary satisfactions of working as a nurse educator?}

IS38 How satisfied are you with your job and performance? Why and in what ways?

IS39 In the last year how many times have you received positive feedback for your performance? In what areas and why? To what extent is this influential in your career as a faculty member?

IS40 Who gave you that feedback? In what areas and why? Does the person who is giving positive feedback influence your satisfaction as a nursing faculty member? In what way?

IS41 What is the nature of the positive feedback you receive? Formal recognition? Informal encouragement/praise? Concrete (as in course release, raise, other)? Psychic (as in good for the self and soul)?

IS42 How much do the different types of satisfaction influence your career 
choice as a nursing educator?

IS43 Do you have any final thoughts or feelings about being a nursing educator? To what extent have these influenced your satisfaction with your career choice? 
APPENDIX B

BACKGROUND QUESTIONNAIRE FOR NURSE EDUCATORS 


\section{Appendix B}

Background Questionnaire for Nurse Educators

To assist with the study, this is a short questionnaire to gather demographic data from nursing faculty prior to the interview.

Please answer each question by placing an $X$ in the box or filling in the appropriate information on the line.

1. Gender: $\square$ Female $\square$ Male

2. College where employed:

3. Age:

4. Marital status: $\square$ Married $\square$ Single $\square$ Domestic Partner

5. Current family responsibilities: $\square$ Dependent children Number of children

$\square$ Caring for one or more adult relatives

6. Years of nursing experience:

7. Years of nursing faculty experience: Full time

Part time

8. Mother's educational attainment: $\square$ Less than high school

$\square$ High School Diploma

$\square$ Some college

$\square$ College Degree

$\square$ Professional or advanced degree

9. Father's educational attainment: $\square$ Less than high school

$\square$ High School Diploma

$\square$ Some college

$\square$ College Degree 
$\square$ Professional or advanced degree

10. My educational attainment:

$\square$ Less than Master's Degree

Working on Master's Degree

$\square$ Master's Degree

Working on Doctorate (e.g., DNP, PhD, EdD

$\square$ Doctoral Degree

THANK YOU VERY MUCH FOR YOUR ASSISTANCE! 
APPENDIX C

NURSE EDUCATOR INTERVIEW SCHEDULE 
Appendix C

\section{Nurse Educator Interview Schedule}

Directions: I am going to ask you a number of open-ended questions. Please give me your honest opinion regarding your teaching experience.

IS1 Tell me when and why you decided on nursing as a profession?

IS2 What influenced your decision to become a faculty member?

IS3 Was there a key person/situation that supported your decision to teach? In what way?

IS4 Anything else you would like to add about personal or background factors that influenced your decision to teach?

IS5 What was your original educational level for nursing licensure? Did this influence in any way your eventual decision to teach? How and why?

IS6 What was your original educational level when you started teaching?

Did this enter into your decision to teach? How and why?

IS7 What are your college's requirements for terminal degrees for promotion and/or tenure? To what extent did this influence your decision to teach?

IS8 Can you compare the flexibility of your teaching position within the college setting and your previous clinical position? Do you consider this a benefit or a burden of teaching? Why?

IS9 What is your sense of this flexibility now (as an experienced faculty member) compared to when you started? What was your perception of this at the time when you first started teaching? Was it influential in your decision to change your career? Why?

IS10 Can you compare the collegiality of your teaching position within the college setting and your previous clinical position? Do you consider this a benefit or a burden of teaching? Why?

IS11 What is your sense of this collegiality now (as an experienced faculty member) compared to when you started? What was your perception of this at the time when you first started teaching? Was this influential in your decision to change your career? Why?

IS12 Compare the organizational climate of your teaching position within the college setting and your previous clinical position? Do you consider this 
a benefit or a burden of teaching? Why?

IS13 What is your sense of this organizational climate now (as an experienced faculty member) compared to when you started? What was your perception of this at the time when you first started teaching? Was it influential in your decision to change your career? Why?

IS14 Can you compare the prestige/status of your teaching position within the college setting and your previous clinical position? Do you consider this a benefit or a burden of teaching? Was it influential in your decision to change your career?

IS15 What is your sense of this prestige/status now (as an experienced faculty member) compared to when you started? What was your perception of this at the time when you first started teaching? Was it influential in your decision to change your career? Why?

IS16 Can you think of any other factors related to work environment that influenced your decision to change from clinical nursing to faculty status? How and why?

IS17 Think about your reasons for changing from the clinical environment to teaching. How do you feel when you teach?

IS18 When you started your career as a nursing faculty member, did you see teaching as a plus or a minus? Why?

IS19 Talk about the extent that you have always loved or disliked school. Did this have any influence on your decision to enter teaching? In what ways?

IS20 How would you describe the rewards of teaching? Has your sense of this changed since you started your teaching career? In what ways?

IS21 How do you feel about your relationship with your nursing students?

IS22 To what extent are your feelings about rewards and relationships connected to the immediacy of the classroom? To long term issues like the nursing shortage? Other factors?

IS23 How do you view the intellectual challenges of staying current with the knowledge about your field? Did this have any influence on your decision to enter teaching? In what ways?

IS24 Can you think of any other factors related to pedagogical considerations that influenced your decision to change from a clinical nurse to a faculty member? 
IS25 Which of these would be the most important factor in your decision to make the change? Why?

IS26 Think back to the first days of your teaching job. What were the expectations with respect to teaching load? Did this influence your decision to enter higher education? In what ways?

IS27 In relation to internal and external service to the college, how did your expectations and those of your supervisor differ? How were they similar?

IS28 Early in your career, what were the expectations for scholarly writing and research? How did that differ from those of your supervisor?

IS29 How much of a concern were the requirements for earning tenure and promotion when you decided to become a faculty member? In what ways?

IS30 Thinking back to your early days as a nurse educator, relate your knowledge of student evaluation. How confident were you? Did this enter into your decision to enter teaching?

IS31 In the early days as a nurse educator, relate your knowledge of clinical evaluation of students. How confident were you? Did this affect your decision to change professions?

IS32 In the early days relate your knowledge of preparation of lecture content. How confident were you?

IS33 In the early days of your teaching position, relate your knowledge of development of examinations. How confident were you?

IS34 In the early days of your teaching position, relate your knowledge of curriculum revision and design. How confident were you?

IS35 In the early days of your teaching position, relate your ability in the use of technology in your education setting. How comfortable were you? How did that differ from your use of technology in the clinical setting?

IS36 Compare your ability to organize and manage your time in your teaching position with your position in the clinical area. How does it differ?

IS37 What is your sense of the collective cost/benefit of these barriers compared to clinical nursing? Has that influenced your decision to remain a nurse educator? 
IS38 How satisfied are you with your job and performance? Why and in what ways?

IS39 In the last year how many times have you received positive feedback for your performance? In what areas and why? To what extent is this influential in your career as a faculty member?

IS40 Who gave you that feedback? In what areas and why? Does the person who is giving positive feedback influence your satisfaction as a nursing faculty member? In what way?

IS41 What is the nature of the positive feedback you receive? Formal recognition? Informal encouragement/praise? Concrete (as in course release, raise, other)? Psychic (as in good for the self and soul)?

IS42 How much do the different types of satisfaction influence your career choice as a nursing educator?

IS43 Do you have any final thoughts or feelings about being a nursing educator? To what extent have these influenced your satisfaction with your career choice?

\section{THANK YOU SO MUCH FOR YOUR TIME!}




\begin{abstract}
APPENDIX D
INFORMATION TO EXPERT PANEL AND PILOT PARTICPANTS
\end{abstract}

LETTER TO EXPERT PANEL MEMBER

EXPERT REVIEW OF BACKGROUND QUESTIONNAIRE FOR NURSE

EDUCATORS

EXPERT REVIEW OF NURSE EDUCATOR INTERVIEW SCHEDULE

LETTER TO PILOT STUDY PARTICIPANT

PILOT STUDY REVIEW OF BACKGROUND QUESTIONNAIRE FOR NURSE EDUCATORS

PILOT STUDY REVIEW OF NURSE EDUCATOR INTERVIEW SCHEDULE 
Appendix D

Letter to Expert Panel Members

Researcher's Address

Expert Panel Member's Address

Dear Expert Panel Member:

I am a student in the Cooperative Doctoral Program at the University of Louisville and Western Kentucky University. I am completing a research project under the direction of Dr. Stephen Miller, College of Education and Behavioral Sciences, Department of Educational Administration, Leadership, and Research at Western Kentucky University.

I am interested in studying factors that affect the nursing shortage. The goal is to complete a dissertation pertaining to career choice of nursing faculty. By studying nursing faculty responses and opinions concerning their decision to teach, I hope to be able to obtain information that may impact the recruitment and retention of nursing faculty.

The purposeful sample will come from associate degree nursing faculty in the state of Kentucky, who have been employed less than five years, 6-10 years, and eleven or more years. I am also looking at the Carnegie Classification size of the college and educational attainment of the faculty person (BSN, working on MSN, MSN, working on Doctorate, or completed EdD/PhD/DNP).

The faculty member will complete a short questionnaire followed by a semistructured interview. I would like feedback into the questions on the background survey as well as the interview. I have attached the research questions mapped to the Background Questionnaire for Nurse Educators and the Nurse Educator Interview Schedule. I would like for you to review these two sets of questions and provide feedback on the forms included. You will find separate copies of the actual background questionnaire and interview schedule so that you can comment directly on the actual instruments, should you desire.

I believe this project is of importance to understanding one aspect of the healthcare crisis today, the nursing faculty shortage. Your feedback will help me improve the elarity and concision of the questions, ensuring the most information from the subjects.

Thank you for your time. Please contact me by phone (270-339-0942) or email (lindajthomas1946@gmail.com) if you are unclear what the task is or if you are unable to participate. Your assistance is appreciated.

Sincerely,

Linda J. Thomas, RN, MSN, CNE 


\section{Appendix D \\ Expert Review of Background Questionnaire for Nurse Educators}

Please respond to the following questions regarding the demographic constructs in the Background Questionnaire for Nurse Educators. Comments may be written directly on the instrument. If additional space is needed, please attach a separate sheet of paper and specify to which item your comments refer.

Please note that the research questions were developed from the literature and theoretical perspective included in Chapter III. If you would like to examine this section, please contact me and I will provide you with a copy.

Thank you again for your time.

The Background Questionnaire for Nurse Educators (Appendix B--attached for your convenience)

1. Introduction:

Is the introductory statement of purpose clear?

2. Format:

Is the format clear?

Easy to follow?

Are the questions clear and understandable?

3. Educational level?

Is the wording appropriate for nursing faculty?

4. Directions?

Are the directions clear and understandable to the faculty member?

5. Feasibility?

Is the instrument practical?

Is the instrument too long? 
6. Do you have any general comments or suggestions on the overall format and presentation of the questionnaire?

7. Do you have any final thoughts about the constructs? Any particular suggestions?

THANK YOU FOR YOUR TIME AND EFFORT! 


\author{
Appendix D \\ Expert Review of Nurse Educator Interview Schedule
}

Please respond to the following questions regarding factors affecting the nurse educators' decision to teach. Comments may be written directly on the instrument. If additional space is needed, please attach a separate sheet of paper and specify to which item your comments refer.

Please note that the research questions were developed from the literature and theoretical perspective included in Chapter III. If you would like to examine this section, please contact me and I will provide you with a copy.

Thank you again for your time.

The Nurse Educator Interview Schedule (Appendix C--attached for your convenience)

1. Introduction:

Is the introductory statement of purpose clear?

2. Format:

Is the format clear?

Easy to follow?

Are the questions clear and understandable?

3. Topics:

Is the wording appropriate for nursing faculty?

Do the questions make sense in terms of content?

4. Directions?

Are the directions clear and understandable to the faculty member?

5. Feasibility?

Is the instrument practical?

Is the instrument too long? 
6. Do you have any general comments or suggestions on the overall format and presentation of the Nurse Educator Interview Schedule?

7. Do you have any final thoughts about the constructs? Any particular suggestions?

THANK YOU FOR YOUR TIME AND EFFORT! 
Appendix D

Letter to Pilot Study Members

Researcher's Address

Pilot Study Member's Address

Dear Pilot Study Member:

I am a student in the Cooperative Doctoral Program at the University of Louisville and Western Kentucky University. I am completing a research project under the direction of Dr. Stephen Miller, College of Education and Behavioral Sciences, Department of Educational Administration, Leadership, and Research at Western Kentucky University.

I am interested in studying factors that affect the nursing shortage. The goal is to complete a dissertation pertaining to career choice of nursing faculty. By studying nursing faculty responses and opinions concerning their decision to teach, I hope to be able to obtain information that may impact the recruitment and retention of nursing faculty.

The purposeful sample will come from associate degree nursing faculty in the state of Kentucky, who have been employed less than five years, 6-10 years, and eleven or more years. I am also looking at the Carnegie Classification size of the college and educational attainment of the faculty person (BSN, working on MSN, MSN, working on Doctorate, or completed EdD/PhD/DNP).

During the study, the faculty member will complete a short questionnaire followed by a semi-structured interview. I would like feedback from the participant's point of view into the process of completing the background questionnaire as well as the interview. After completing both the questionnaire and the interview, I will ask you to give feedback about the wording, format, and content of the instruments.

I believe this project is of importance to understanding one aspect of the healthcare crisis today and the nursing faculty shortage. Your feedback on the questions will help me improve the clarity and concision of the questions, ensuring the most information from the subjects.

Thank you for your time. Please contact me by phone (270-339-0942) or email (lindajthomas1964@gmail.com) if you are unclear what the task is or if you are unable to participate. Your assistance is appreciated.

Sincerely,

Linda J. Thomas, RN, MSN, CNE 


\section{Appendix D}

Pilot Study Participants' Review of Background Questionnaire for Nurse Educators

After completing the Background Questionnaire for Nurse Educators prior to the interview, please respond to the following questions regarding factors affecting the nurse educators' decision to teach. Comments may be written directly on the instrument. If additional space is needed, please attach a separate sheet of paper and specify to which item your comments refer.

Please note that the research questions were developed from the literature and theoretical perspective included in Chapter III. If you would like to examine this section, please inquire and I will provide you with a copy.

Thank you for your time in answering the questions for the interview and providing feedback in the process.

The Background Questionnaire for Nurse Educators (included for your convenience)

1. Introduction:

Was the introductory statement of purpose clear and easily understood?

2. Format:

Was the format clear?

Easy to follow?

Were the questions clear and understandable?

3. Topics:

Was the wording appropriate for nursing faculty?

Did the questions make sense in terms of content?

4. Directions:

Were the directions clear and understandable?

5. Feasibility:

Is the instrumental practical?

Is the instrument too long?

What specifically would you suggest to shorten the questionnaire?

6. Do you have any comments or suggestions on the overall format and presentation of the Background Questionnaire for Nurse Educators?

THANK YOU FOR YOUR TIME AND EFFORT! 


\section{Appendix D}

Pilot Study Participants' Review of Nurse Educator Interview Schedule

After completing the Nurse Educator Interview Schedule with the researcher, please respond to the following questions regarding factors affecting the nurse educators' decision to teach. Comments may be written directly on the instrument. If additional space is needed, please attach a separate sheet of paper and specify to which item your comments refer.

Please note that the research questions were developed from the literature and theoretical perspective included in Chapter III. If you would like to examine this section, please inquire and I will provide you with a copy.

Thank you for your time in answering the questions for the interview and providing feedback in the process.

The Nurse Educator Interview Schedule (included for your convenience)

7. Introduction:

Was the introductory statement of purpose clear and easily understood?

8. Format:

Was the format clear?

Easy to follow?

Were the questions clear and understandable?

9. Topics:

Was the wording appropriate for nursing faculty?

Did the questions make sense in terms of content?

10. Directions: 
Were the directions clear and understandable?

11. Feasibility:

Is the instrumental practical?

Is the instrument too long?

What specifically would you suggest to shorten the questionnaire?

12. Do you have any comments or suggestions on the overall format and presentation of the Nurse Educator Interview Schedule?

THANK YOU FOR YOUR TIME AND EFFORT! 
APPENDIX E

LETTER TO NURSE ADMINISTRATOR

CONTACT LETTER TO NURSING FACULTY MEMBER

LETTER OF CONFIRMATION TO FACULTY MEMBER

LETTER OF NOTIFICATION OF NONPARTICIPATION 
Appendix E

Letter to Nurse Administrator

Researcher's address

Nurse Administrator's address

Dear (Nurse Administrator's name):

I am currently a doctoral student in the Cooperative Doctoral Program between the University of Louisville and Western Kentucky University. I am a former Division Chair and Associate Degree Nursing Program Coordinator at Madisonville Community College. I am presently completing my dissertation under the direction of Dr. Stephen Miller, College of Education and Behavioral Sciences, Department of Educational Administration, Leadership, and Research at Western Kentucky University.

I am interested in studying factors that affect the nursing faculty shortage. The goal is to complete a dissertation pertaining to career choice of nursing faculty. By studying nursing faculty responses and opinions concerning their decision to teach, I hope to be able to provide information that may impact the recruitment and retention of nursing faculty.

I would like to interview nursing faculty members based on experience, size of institution, and level of education. I hope to interview one faculty member from each of the 20 associate degree nursing programs. I have completed a grid so that the faculty member from each institution contributes to an overall purposeful sample as representative as possible. If you could identify faculty who meet these guidelines for your program's place in the grid, I will send an invitation to each individual to participate in the study. I will then select one person to interview (including the completion of a short questionnaire) from those who respond positively.

The characteristics of the grid for the purposeful sample include the following variables: years of experience (1-5, 6-10, or more than 10 years); size of the college (small or medium/large); and educational level (BSN, working on MSN, MSN, working on $\mathrm{PhD}$ or $\mathrm{DNP}$, or $\mathrm{PhD} / \mathrm{DNP}$ ). The descriptor depicts the particular characteristics that the person from your institution should meet. If there are several faculty members in your institution who meet these criteria, please select four that are representative of that specific set of characteristics. If there are four or less, please identify all who meet the criteria.

I hope that you will be willing to assist me with this project; I appreciate your time and attention. As the co-investigator, I will adhere to the ethical standards of research consistent with the law and regulations on human subjects. This includes ensuring privacy and confidentiality of the interview and raw data collected to the extent possible. This means that each person who participates in this study will be required to sign a consent form.

I will call within three days to check to see if you have questions or concerns, or whether there is anything I can do to facilitate your role in this research project. 
In order to satisfy procedures for human subjects, please follow this protocol.

1. I have included a copy of a "Contact Letter to Nurse Faculty Member" (attached). Please copy so that you can send one to each faculty member (up to four) who meets the criteria for experience, educational level, and size as specified for your program.

2. Please script a memo to attach to the enclosed contact letter, encouraging your faculty to volunteer to participate.

3. Send your memo and the contact letter to each faculty member whom you select in your college.

4. I will interview one person who responds affirmatively to the invitation to participate in the study. If more than one person responds, I will select one person who best completes the sample of 20 based on demographic factors such as gender, ethnicity, or rural/urban background.

5. If more than one person responds positively, I will notify those not selected, explaining to them why only one could be interviewed.

6. If no faculty member responds positively, I will contact you regarding the possibility of a second invitation to participate. your faculty.

Thank you again for your consideration. I look forward to working with you and

Sincerely,

Linda J. Thomas MSN, RN, CNE 
Appendix E

Contact Letter to Nursing Faculty Member

Researcher's address

Dear Nursing Faculty Member:

You are being invited to participate in a qualitative research project entitled "Factors Affecting Nurse Educators' Decision to Teach." This study partially fulfills doctoral dissertation requirements. Dr. Stephen K. Miller, the advisor for this research study, is affiliated with the College of Education and Behavioral Sciences, Department of Educational Administration, Leadership, and Research at Western Kentucky University.

This study will be conducted through the guidance of Western Kentucky University and the University of Louisville, and the associate degree nursing programs in the state of Kentucky. The goal is to accommodate each participant by completing the interview session at each college.

Despite the economic recession, the number of nursing positions continues to grow and the nursing shortage continues to remain a crisis in healthcare. Concomitantly, the nursing faculty shortage threatens the opportunity to provide a sufficient number of nurses to care for the nation's population now and in the future. Thus, the purpose of the research study is to provide insight into the career choice of nurses to teach in associate degree nursing programs.

The study is designed to collect data from 20 associate degree nursing faculty members in Kentucky. Your nurse administrator forwarded me names who meet selected characteristics based on experience, education and size of program. I will select and contact the individual who best completes the overall grid of 20 positions. If you volunteer and are not selected, I will notify you. Please understand that I appreciate your willingness to participate whether you are selected or not.

Two instruments will be used to gather information. Prior to participation, you will be required to complete a consent form. Then, before the interview, you will be asked to complete a short questionnaire. A semi-structured format with open-ended questions will be used for the interview. The approximately one hour recorded session will be conducted in person at a mutually agreed upon time.

Be assured that there are no physical, psychological, financial, or legal risks to you or any participant associated with the study. The benefits gained from your participation will provide information about the reasoning and incentives regarding the decision by nurses to teach in associate degree nursing programs. This understanding should help in developing strategies to increase the number of nursing faculty.

Absolute confidentiality cannot be guaranteed; however, data will be held in confidence to the extent permitted by law. All information collected may be reviewed by Dr. Miller and/or others associated with the research study. Your identity will not be revealed should this study be published.

Your participation in this research study is voluntary. You are free to withdraw your consent at any time without penalty. You are free to decline to answer any particular question that may make you feel uncomfortable:

If you have any questions, please call me (home, 270-322-8914) or e-mail 
(lindajthomas1946@gmail.com) or Dr. Miller (work, 270-745-4890). You may call the Human Studies Committee offices at Western Kentucky University (270-745-2129) or University of Louisville (502-852-5188) where you will be given an opportunity to discuss any question about your rights as a research subject, in confidence, with a member of the respective Committee. These are independent committees composed of faculty and staff of Western Kentucky University and at the University of Louisville. The committees have reviewed this study.

Sincerely, Linda J. Thomas MSN, RN, CNE 
Responding to the Invitation

Please fill out the following information and return to your nurse administrator. She will forward this form to me.

Alternatively, you may email the information to me (lindajthomas1946@ gmail.com).

Name (Please Print)

Address

Telephone (including area code)

Email (Please print)

I am responding to the invitation to participate in the research project, "Factors Affecting Nurse Educators' Decision to Teach." I understand that I will receive a Consent Form to sign prior to participation. The researcher, Linda Thomas, will contact me for a mutually agreeable time/place to meet.

Signature

Date 


\author{
Appendix E \\ Letter of Response to Faculty Member
}

Dear Nurse Faculty Member,

This letter is to confirm your selection to the research study, "Factors Affecting Nurse Educators' Decision to Teach." You are now officially enrolled as a participant in the study. I appreciate your willingness to be a part of this important endeavor and will be contacting you by phone or e-mail to finalize the date, time, and location for the interview. You can anticipate somewhat around an hour for the process of completing the Background Questionnaire for Nurse Educators and the Nurse Educator Interview Schedule. I will bring the consent form with me for you to sign.

Again, I would like to thank you for your participation in this study. I look forward to the meeting and having your input into this project.

Sincerely,

Linda J. Thomas MSN, RN, CNE 
Appendix E

Letter of Notification of Nonparticipation

Dear Nurse Faculty Member:

This letter is to thank you for volunteering to participate in the research study, "Factors Affecting Nurse Educators' Decision to Teach." More than one faculty member from your program met the criteria and agreed to participate. The study interviews one person from each nursing program. To assure diversity in the collection of data, informal factors such as gender, ethnicity, and rural/urban background were also considered. Based on those criteria, another individual from your program was selected.

I appreciate your willingness to provide insight into this important subject and if you wish, I will provide you a summary of the results. Thank you again for your interest in the research study.

Sincerely,

Linda J. Thomas MSN, RN, CNE 
APPENDIX F

STUDY CONSENT FORM 


\section{INFORMED CONSIENT DOCUMENT}

\section{FACTORS AFFHCTING NURSE EDUCATORS' DECISION TO TEACH}

You are being invited to participate in a qualitative research project entitled "Factors Affecting Nurse Educators Decision to Teach." This study partially fulfills doctoral dissertation requirements. Dr. Stephen K. Miller. the advisor for this research study, is affiliated with the College of Education and Behavioral Sciences. Department of Educational Administration, I.eadership. and Research at Western Kentucky University

This study will be conducted through the guidance of Western Kentucky University and the Iniversity of louisville, and the associate degree nursing programs in the state of Kentucky. The goal is to accommodate each participant by completing the interview session at their home college.

The study is designed to collect data from 20 associate degree nursing faculty members in Kentucky. You have been selected from your college because you meet the predetermined qualifications of the study. The interview is being conducted at a location in your college at the time and place of your choosing.

Despite the economic recession, as I am sure that you are aware of, the number of nursing positions continues to grow and the nursing shortage continues to remain a crisis in healthcare. Concomitantly, the nursing taculty shortage threatens the opportunity to provide a sufficient number of murses to care for the nation's population now and in the future. Thus, the purpose of the research study is to provide insight into the career choice of nurses to teach in associate degree nursing programs.

Two instruments will be used to gather information, the Background Questionnaire for Nurse Educators and the Nurse Educator Interview Schedule. You will be asked to complete the background questionnaire prior to the interview. Then. a semi-structured format with open-ended questions will be used for the interview. The approximately one hour recorded session will be conducted in person, by me. Linda J. Thomas. I will be contacting you after the recorded session has been processed so you will be able to verify the information from the interview.

Be assured that there are no physical, psychological, financial, or legal risks to you or any participant associated with the study. The benefits gained from your participation will provide information about the reasoning and incentives regarding the decision by nurses to teach in associate degree nursing programs. This understanding should help in developing strategies to increase the number of nursing faculty.

Absolute contidentiality cannot be guaranteed: however. data will be held in confidence to the extent permitted by law. Your unique number on your questionnaire and interview will be recorded next to your name on a register that will be kept separate from the two instruments. All information collected may be reviewed by Dr. Miller and/or others associated with the research study. Your identity will not be revealed should this study be published.

Your participation in this research study is voluntary. You are free to withdraw your consent at any time without penalty. You may refuse to participate without incurring any penalty or losing any

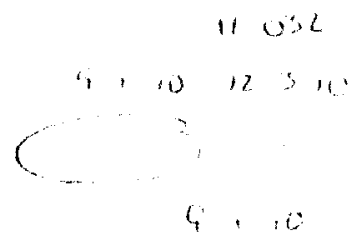


benefits to which you are otherwise entitled. You are free to decline to answer any particular question that may make you feel uncomfortable.

If you have concerns or complaints about the study or the study staff, you have three options:

1. You may contact Dr. Stephen Miller (work, 270-745-4890) or Linda J. Thomas (home, 270$322.8914)$.

2. If you have any questions about your rights as a study subject, questions, concerns or complaints, you may call the Human Subjects Protection Program (Office (HSPPO) at (502) 852-5188, or Paul Mooncy, Ilunan Protections Administrator. Western Kentucky I niversity. 270-745-4652. You may discuss any questions about your rights as a subject, in secret. with a nember of the Institutional Review Boad (IRB) or the HSPPO staff. The IRB is an independent committee composed of members of the University community, staff of the institutions. as well as lay members of the community not connected with these institutions. The IRB has reviewed this study.

3. If you want to speak to a person outside the University, you may call 1-877-852-1167. You will be given a chance to talk about any questions, concerns, or complaints in secret. This is a 24 hour hot line answered by people who do not work at the Lniversity of Louisville.

This paper tells you what will happen during the study if you choose to take part. Your signature means that this study has been discussed with you, that your questions have been answered. and that you will take part in the study. This informed consent document is not a contract. You are not giving up any legal rights by signing this informed consent document. You will be given a signed copy of this paper to keep for your rccords.

Printed Name of Participant

Date

linda J. Thomas, Researcher

Date

I understand my responses during the Nurse Educator Interview Schedule will be tape recorded. transcribed. and the audio tapes will be destroyed following completion of the study. I give permission to tape record my responses during the interview.

Signature of Paricipant

THE DATED APPROVAL. ON THIS CONSENT FORM INDICATES THAT THIS PROJECT IIAS BEEN RI:VII:WIED AND APPROVIED BY THE WISSTFRN KIENTUCKY UNIVIERSTYY IRB

Paul Mooney, lluman Protections Administrator MLLLPHONL: (270) 745-4652

$$
11 \text { \\2 }
$$$$
\text { i } 10 \quad 12: 11
$$
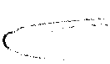

$, 1,1$, 
APPENDIX G

HUMAN SUBJECTS DOCUMENTS

LETTER OF APPROVAL UNIVERSITY OF LOUISVILLE

LETTER OF APPROVAL WESTERN KENTUCKY UNIVERSITY 


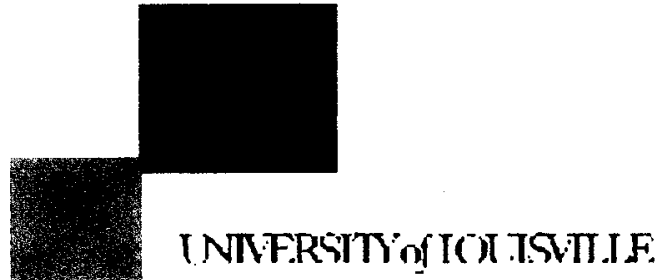

To: $\quad$ Miller, Stephen

From: The University of Louisville Institutional Review Board (IRB)

Date: $\quad$ Tuesday, September 07, 2010

Subject: IRB Correspondence

Tracking \#: 10.0406

Title: $\quad$ Factors Affecting Nurse Educators' Decision To Teach
Mstrmumoul REVIEN BOAFos

inversy of Lousus:

Nedcemer One, sure 200

501 E Erovingy

Loulsile, Kentucty 40202-1790

Once: $\quad 502-852-5188$

Fax $502-852-2164$

This study was reviewed on 09/03/2010 and determined by the chair of the Institutional Review Board that the study is exempt according to 45 CFR 46.101(b) under category (2) Research involving the use of educational tests (cognitive, diagnostic, aptitude, achievernent), survey procedures, interview procedures or observation of public behavior, unless: (i) information obtained is recorded in such a manner that human subjects can be identified, directty or through identifiers linked to the subjects; and (ii) any disclosure of the human subjects' responses outside the research could reasonably place the subjects at risk of criminal or civil liability or be damaging to the subjects' financial standing, employability, or reputation. The study is exempt only if information that could identify subjects is not recorded.

Since this study has been found to be exempt, no additional reporting, such as submission of Progress Reports for continuation reviews, is needed. If your research focus or activities change, please submit a Study Amendment Request Form to the IRB for review to ensure that the study still meets exempt status. Best wishes for a successful study. Please send all inquires and electronic revised/requested items to our office email address at hsppofc:louisville.edu.

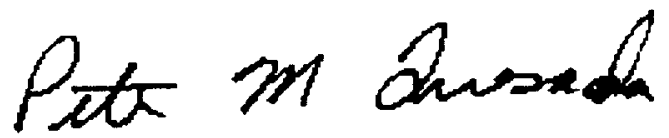

Board Designee: Quesada, Peter 


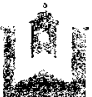 \\ WKU.}

A LEADING AMFRICAN UATVERSIY WHHH INTERNATIONAL RLACH

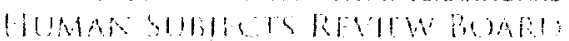

In future correspondence, please refer to HS1 1-032, September 1, 2010

Dr. Steve Miller

Linda $\mathbf{J}$. Thomas

Educational Administration, Leadership, and Research

WKU

Dr. Steve Miller \&

Linda J. Thomas:

Your research project, What Factors Influenced the Career Choice of Nurse Educators Currently Teaching in Kentucky Associate Degree Nursing Programs?, was reviewed by the HSRB and it has been determined that risks to subjects are: (1) minimized and reasonable; and that (2) research procedures are consistent with a sound research design and do not expose the subjects to unnecessary risk. Reviewers determined that: (1) benefits to subjects are considered along with the importance of the topic and that outcomes are reasonable; (2) selection of subjects is equitable; and (3) the purposes of the research and the research setting is amenable to subjects' welfare and producing desired outcomes; that indications of coercion or prejudice are absent, and that participation is clearly voluntary.

1. In addition, the IRB found that you need to oriem participants as follows: (1) signed informed consent is required; (2) Provision is made for collecting, using and storing data in a manner that protects the safety and privacy of the subjects and the confidentiality of the data. (3) Appropriate safeguards are included to protect the rights and welfarc of the subjects.

This project is therefore approved at the Expedited Review Level until December 3, 2010.

2. Please note that the institution is not responsible for any actions regarding this protocol before approval. If you expand the project at a later date to use other instruments please re-apply. Copies of your request for human subjects review, your application, and this approval, are maintained in the Office of Sponsored Programs at the above address. Please report any changes to this approved protocol to this office. A Continuing Review protocol will be sent to you in the future to determine the status of the project. Also, please use the stamped approval forms to assure participants of compliance with The Office of Human Research Protections regulations.

Sincerely,

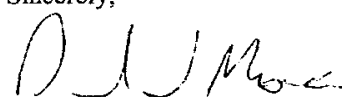

Paul J. Mooney, M.S.T.M.

Compliance Coordinator

Office of Sponsored Programs

Western Kentucky University

cc: HS file number Miller HS 1 1-032

$11-032$

$4110: 12,10$

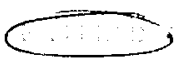

$4: 10$

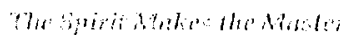

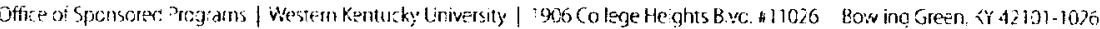

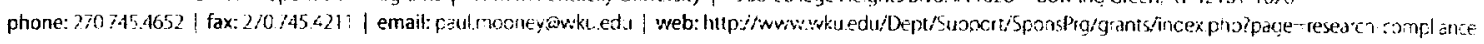

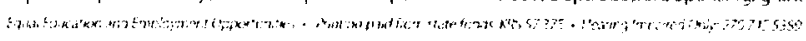


APPENDIX H

INTERVIEW SITE MAP 


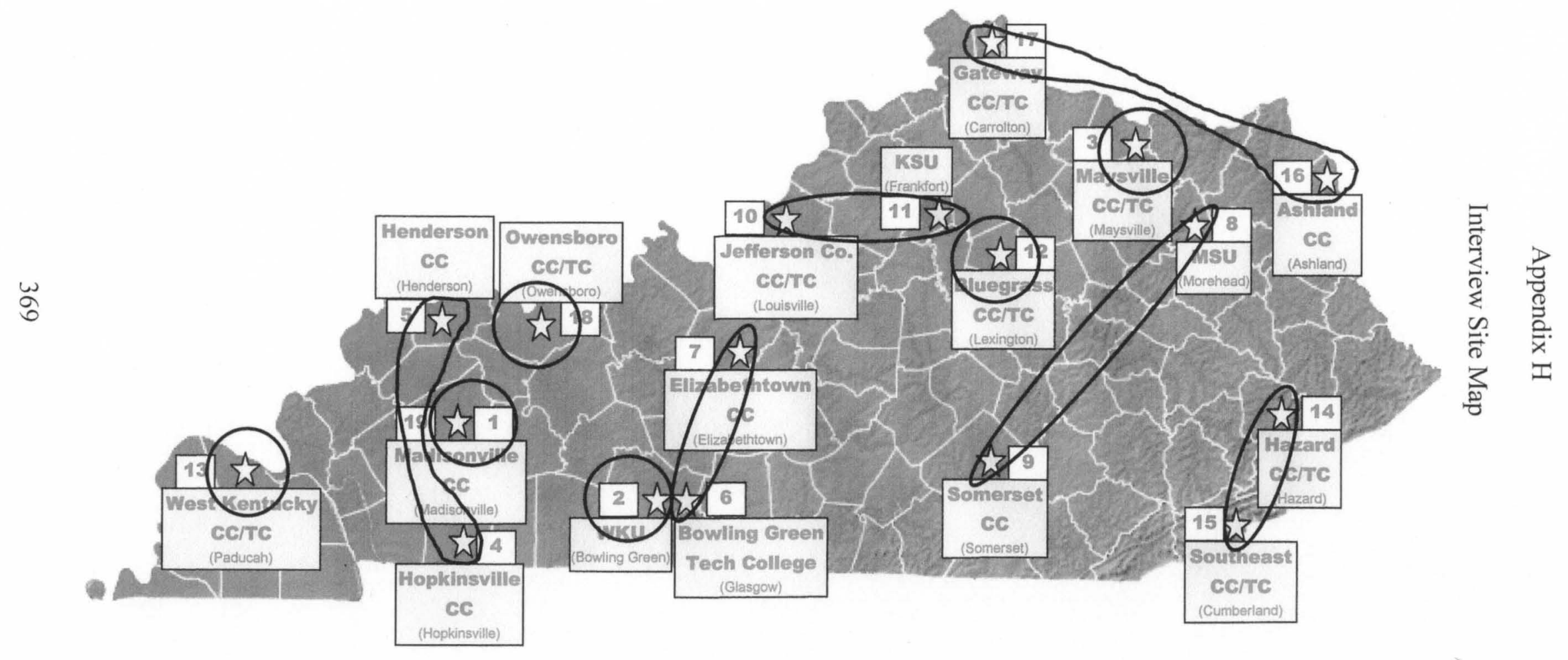




\section{APPENDIX I}

CODED MATRIX ANALYTICAL TOOL

SUMMARIZED IS QUESTIONNAIRE RESPONSE MATRIX 


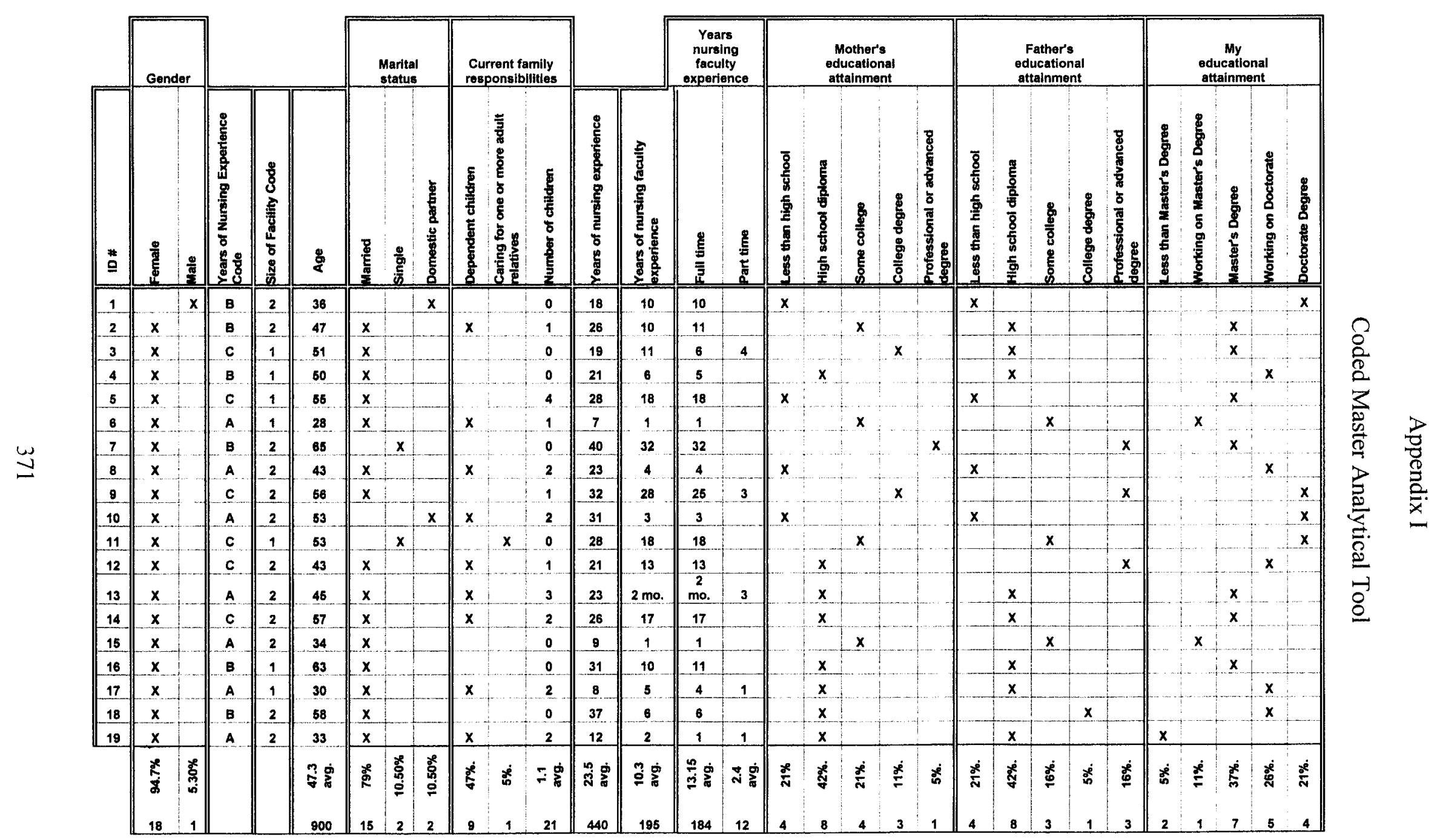


ISI "Tell me when and why you decided on nursing as a profession:

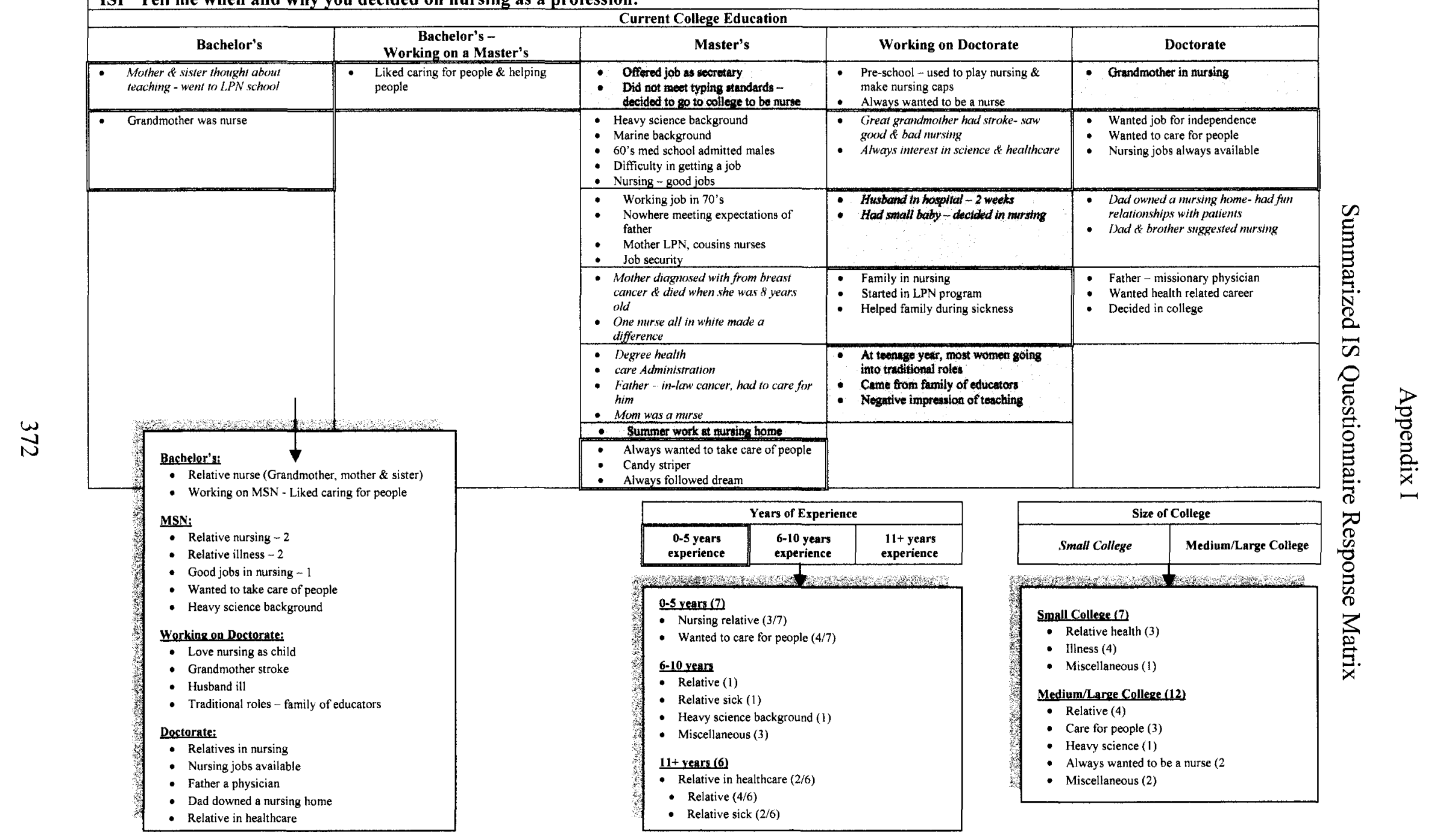




\title{
CURRICULUM VITAE
}

\author{
NAME: $\quad$ Linda Joyce Thomas \\ ADDRESS: 1368 Sunset Road \\ Hanson, KY 42413 \\ DOB: $\quad$ Warrensburg, Missouri - August 27, 1946 \\ EDUCATION \\ \& TRAINING: $\quad$ University of Michigan \\ 1964-1966 \\ BSN - University of Kansas \\ 1966-1969 \\ MSN - University of Evansville \\ $1982-1985$ \\ Certified Nurse Educator \\ 2008
}

AWARDS: NISOD Excellence Award in Teaching

Madisonville Community College New Horizon's Excellence Award

Loman C. Trover MD Award for Outstanding Contributions to Health Care

Educator of the Year - Madisonville/Hopkins County, KY Chamber of

Commerce

PROFESSIONAL SOCIETIES:

Sigma Theta Tau - Nursing Honor Society

Phi Kappa Phi - Educational Honorary

American Nurses Association

Kentucky Nurses Association

National League for Nursing

NATIONAL MEETING PRSENTATIONS:

Career Pathways in Nursing, League for Innovation - Denver, CO

Career Pathways in Nursing - Austin, TX 